\title{
Less than meets the eye: the functional relevance of early visual cortex across the visual domain
}

Citation for published version (APA):

Jacobs, C. J. H. (2012). Less than meets the eye: the functional relevance of early visual cortex across the visual domain. [Doctoral Thesis, Maastricht University]. Maastricht University Press. https://doi.org/10.26481/dis.20120928cj

Document status and date:

Published: 01/01/2012

DOI:

10.26481/dis.20120928cj

Document Version:

Publisher's PDF, also known as Version of record

\section{Please check the document version of this publication:}

- A submitted manuscript is the version of the article upon submission and before peer-review. There can be important differences between the submitted version and the official published version of record.

People interested in the research are advised to contact the author for the final version of the publication, or visit the DOI to the publisher's website.

- The final author version and the galley proof are versions of the publication after peer review.

- The final published version features the final layout of the paper including the volume, issue and page numbers.

Link to publication

\footnotetext{
General rights rights.

- You may freely distribute the URL identifying the publication in the public portal. please follow below link for the End User Agreement:

www.umlib.nl/taverne-license

Take down policy

If you believe that this document breaches copyright please contact us at:

repository@maastrichtuniversity.nl

providing details and we will investigate your claim.
}

Copyright and moral rights for the publications made accessible in the public portal are retained by the authors and/or other copyright owners and it is a condition of accessing publications that users recognise and abide by the legal requirements associated with these

- Users may download and print one copy of any publication from the public portal for the purpose of private study or research.

- You may not further distribute the material or use it for any profit-making activity or commercial gain

If the publication is distributed under the terms of Article $25 \mathrm{fa}$ of the Dutch Copyright Act, indicated by the "Taverne" license above, 


\section{LESS THAN MEETS THE EYE}

The functional relevance of early visual cortex across the visual domain 


\section{Colofon}

Cover design by Christianne Jacobs

Production: Datawyse | Universitaire Pers Maastricht

(C) Copyright Christianne Jacobs, Maastricht 2012

ISBN 9789461591746 


\title{
LESS THAN MEETS THE EYE
}

\section{The functional relevance of early visual cortex across the visual domain}

\author{
PROEFSCHRIFT \\ ter verkrijging van de graad van doctor aan de Universiteit Maastricht, \\ op gezag van de Rector Magnificus, prof. dr. L.L.G. Soete \\ volgens het besluit van het College van Decanen, \\ in het openbaar te verdedigen op
}

vrijdag 28 september 2012 om 12.00 uur

door

Christianne Julienne Henriëtte Jacobs

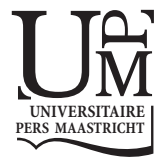




\section{Promotores:}

Prof. dr. A.T. Sack

Prof. dr. R. Goebel

\section{Beoordelingscommissie:}

Prof. dr. P. de Weerd (voorzitter)

Prof. dr. E. Formisano

Dr. V. Goffaux (Université du Luxembourg)

Prof. dr. G. Thut (University of Glasgow)

Dr. K. Uludag

Het in dit proefschrift beschreven onderzoek werd mede mogelijk gemaakt door een subsidie van de Nederlandse organisatie voor Wetenschappelijk Onderzoek (NWO, nr. 400-07-048 toegekend aan Alexander T. Sack) 


\section{CONTENTS}

Chapter 1

General introduction

Chapter 2

Visual awareness suppression by pre-stimulus brain stimulation; a neural effect

Chapter 3

Temporal dynamics of early visual cortex involvement in behavioral priming

Chapter 4

Topographic contribution of early visual cortex to short-term memory consolidation

Chapter 5

Visual evoked potentials under conditions or pre-stimulus TMS

Chapter 6

General discussion

Summary

Samenvatting

Acknowledgments

Curriculum Vitae 


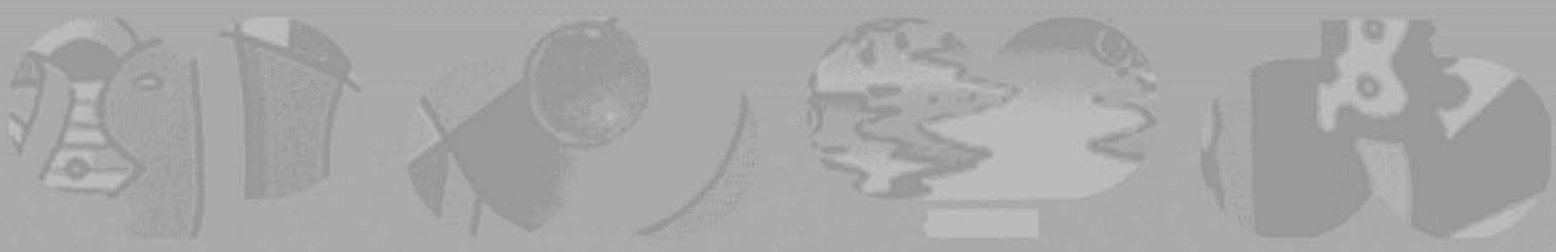

\section{Chapter 1 General introduction}
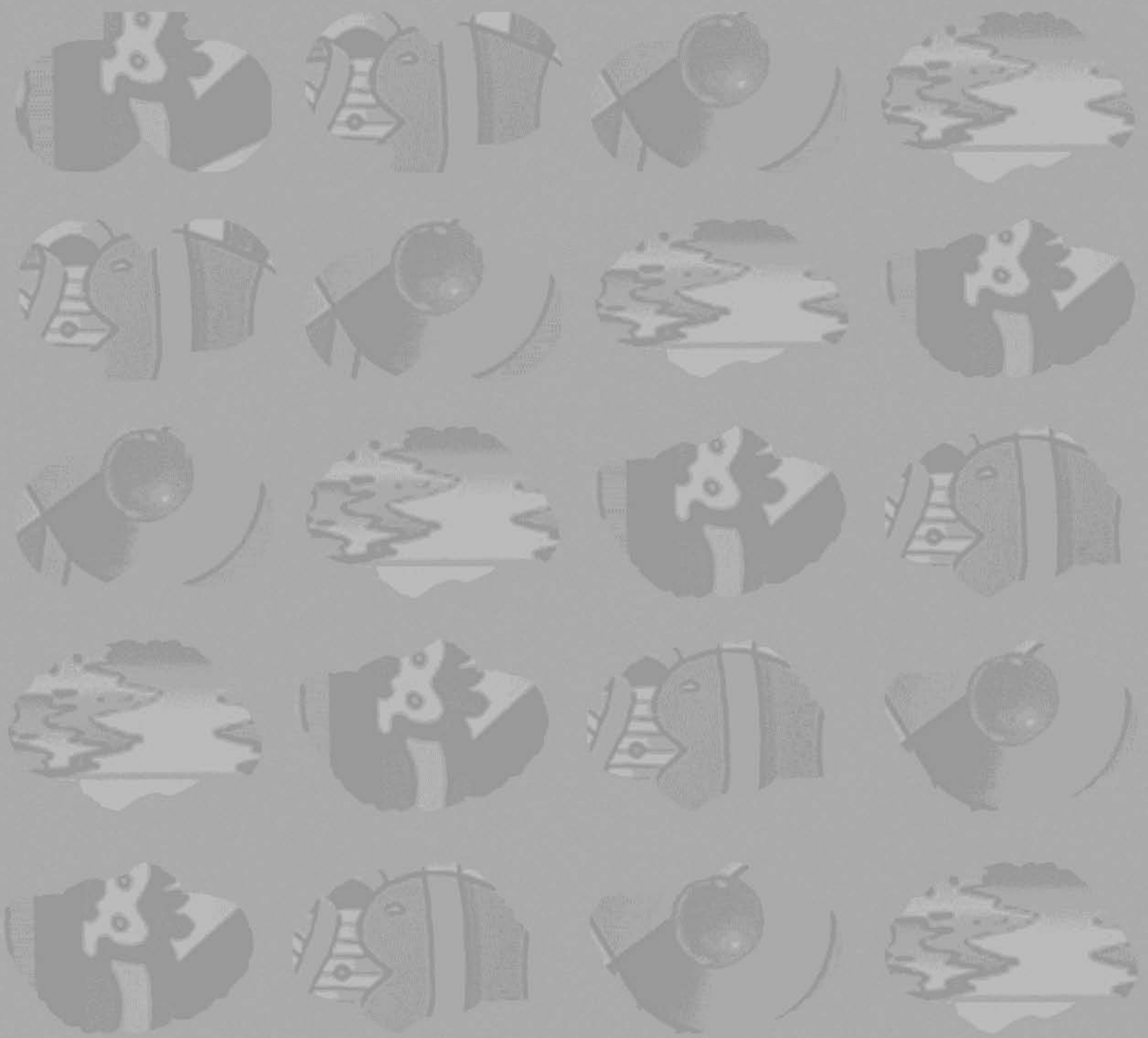
Vision helps us navigate our way through life. Signaling the red sign on a traffic light is as crucial to our survival nowadays as signaling a predator was at earlier stages in our evolution. Besides its functional role, vision enriches our lives in many more ways. Experiencing Vincent van Gogh's Sunflowers or seeing the smiling face of a child are examples of circumstances when vision literally and figuratively adds color to our lives. The capability to see is something we share with many species down the evolutionary ladder. It is amazing that a skill as intricate as eyesight has developed so early on in evolutionary history. The complexity of visual perception becomes clear especially in animals which dedicate large parts of their brains to it. In humans, cognitive skills like language and reasoning developed over the course of evolution. Nevertheless, still a quarter of the most sophisticated part of our brains, i.e. the (neo)cortex, is engaged in the analysis of incoming visual stimuli.

The exact way in which the human brain deals with visual input is a puzzle that neuroscientists have tried to solve over many decades now. In general, one can say that by gaining knowledge on the normal functioning of the brain, we might eventually find a way to deal with the dysfunctioning brain; a statement that is also applicable to visual perception. Losing the ability to perceive the visual richness of our environment is a distressful event. Unfortunately, for some people this is the reality they are confronted with every day. Blindness can have multiple causes, some of which have an origin in the optical organs, the eyes, or in the optical tract transferring information from the eyes to the brain. In these cases, the part of neocortex responsible for processing of visual information is still intact, but the light reflected by the objects in the environment simply cannot reach it. Treatment might be possible, but the success thereof depends on the way the brain handles the renewed visual input. The removal of congenital cataract, for example, might only succeed when the patient is treated at a young age and the brain is still flexible enough to adapt to the new situation (Maurer, Lewis, \& Mondloch, 2005; Ostrovsky, Andalman, \& Sinha, 2006; Thomas, 2011).

Recently, attempts have been made to develop visual prostheses. Analogous to the cochlear implant in hearing, visual cortical implants replace the defected sensory organ, e.g. by an infrared camera that sends its input directly to the visual brain, and so creates a crude visual percept (Merabet, Rizzo, Amedi, Somers, \& Pascual-Leone, 2005; Tehovnik, Slocum, Smirnakis, \& Tolias, 2009). In order to successfully translate the image into a percept comprehensible to the patient, knowledge about the way in which the visual system deals with natural inputs is vital.

Neuroscientific research has taught us much about the workings of the human visual brain. Now science has taken up the challenge of mimicking this by artificially stimulating the brain to create the experience of vision in people for whom this was considered to be lost forever. Even though this example clearly shows what research on visual perception in the brain has brought us so far, we have not yet cracked the visual code altogether. Notwithstanding the fact that we by now have much insight into the parts of the brain involved in visual analysis, and even the particular types of information that these areas process, there is still a lot to learn when it comes to the way the brain executes 
certain visual tasks involving different perceptual or cognitive demands. Research on the normal functioning of the human visual system is crucial in reaching these goals. Nonetheless, also damaged brains have proven to be extremely valuable sources of this knowledge, because the specific behavioral deficits they cause can be linked to the regions of injury. However, there are also numerous drawbacks with regard to lesion studies, among which are the small patient numbers, the absence of pre-lesion data, and the brain's ability to adapt itself to injury, so that the functional impact of the lesion is minimized. Fortunately, the method of transcranial magnetic stimulation (TMS) allows conclusions about functional brain-behavior relationships without suffering the typical drawbacks observed in case studies of lesioned patients.

In this thesis, an attempt will be made to disentangle different visual processes and the way they are embedded in the human visual system. Hereto, TMS has been employed in order to transiently and reversibly perturb the visual system of healthy individuals, and to study the resultant effects on behavior and neural functioning. In the first of the following introductory sections, multiple visual processes are presented that have been dissociated behaviorally, and are thus prone to have diverse underlying neural mechanisms. Secondly, an overview of the various cortical visual pathways is given, and the current views with regards to their functions are evaluated in the light of recent neuroscientific research. Thirdly, the research method of TMS is introduced and the physical and physiological principles thereof are explained, followed by a detailed assessment of the contributions of TMS to the neuroscience of vision. Finally, an overview of the specific research line investigated in this thesis is provided.

\section{PSYCHOPHYSICS OF VISION}

All behavior is grounded in the brain. Consequently, two tasks showing deviant behavioral patterns, most probably have different neural signatures as well. The psychological field of psychophysics investigates the relationship between the physical properties of stimuli and the way, in which they modulate behavior. The precise nature of the psychophysical differences between two tasks can direct the search for their neural underpinnings. Doing the intimate relationship between brain and behavior justice, any comprehensive neural model of visual perception should ultimately explain human behavior as captured in psychophysical results. In the same fashion, the abnormal behavioral profile of patients that suffer from damage to their cortical visual system should be accounted for. To close the circle, a full model of visual perception would ideally lead to new hypotheses, which can subsequently be tested in a psychophysical set-up. 


\section{Unconscious vision}

Over the course of history, a recurrent topic in philosophy, psychology and more recently in neuroscience has been the origin of consciousness. We all continuously experience that we are conscious. When reading this text, for example, you are conscious of the letters on the page, the meaning of the words and perhaps even of other stimuli in your environment, like the sounds of cars passing by. But how or why we are conscious remains puzzling. Is it possible to tear visual awareness apart from other visual processes? If so, how does the brain achieve the generation of visual awareness? The first clue to an answer stems from patients who suffer a lesion to a part of their visual system.

The human cortical visual system is located in the occipital lobe on the posterior side of the brain (see Figure 1). Cortical blindness can occur when the brain's visual system has been damaged, e.g. as a result of trauma. When a lesion befalls the earliest cortical visual area, the primary visual cortex (V1), patients experience a blind spot in their visual field which is called scotoma. A fascinating phenomenon has been discovered in some of these blind patients. As one would expect, when asked whether they perceive any visual stimulation within their scotoma, the patients deny this. However, when forced to make a decision about, for instance the position or direction of motion of a small spot of light, they perform better than to be expected if they were purely guessing (Weiskrantz, 2009); a finding which is as remarkable to the patients themselves as to the experimenter. This neurological syndrome is called 'blindsight'. The name sounds like a contradiction in terms, but in fact it properly reflects the subjective blindness of the patients existing in concert with the objective sight which is still present and is demonstrated by their performance on visual tasks.

The notion that it is feasible to make fairly accurate decisions about stimuli without consciously perceiving them is a counterintuitive one, as we strongly feel that our behavior and our experience go hand-in hand. Nevertheless, under particular experimental conditions, a similar dissociation can also be observed in healthy individuals, which is then referred to as subliminal perception (Lau \& Passingham, 2006). This literally means below-threshold perception; the threshold here being the threshold for visual awareness. Subliminal visual stimuli are either perceptually weakened by decreasing their size, duration or contrast-to-background, or they are actively suppressed by presenting a second, so-called mask stimulus. Most commonly, both strategies of visual suppression are employed together. Within the field of psychophysics, masking paradigms have been utilized to directly investigate matters of visual awareness, such as the minimal requirements for conscious perception (Enns \& Di Lollo, 2000). Next to the perceptual strength of the visual stimulus, stimulus timing appears essential here, because masking effectiveness depends heavily on the relative duration and stimulus-onset asynchrony (SOA) of target and mask stimuli.

The concept of subliminal perception is intriguing, because it reflects the fact that our brains can 'know' more than we consciously experience. This begs to question how our decisions are influenced by these unconscious (neural) 
processes. The extent to which subliminal stimuli are processed is usually measured implicitly as a behavioral effect on a later stimulus.

\section{The behavioral impact of unconscious vision}

James Vicary reported in 1957 that he had made people buy Coca-Cola. He claimed that he had presented cinema audiences with the message "drink CocaCola" so briefly that the words were imperceptible. Amazingly enough, he counted an increase in Coca-Cola sales during the movie break, which he attributed to the unconscious influence of the subliminal message. The discovery of subliminal influences on consumer behavior caused great excitement at the time, and for obvious reasons. Vicary's study turned out to be a hoax, and the experiment had in fact never really been executed. Although replicating Vicary's 'findings' proved difficult, recently new studies have revealed that, for example, consumer's brand choice can in fact be modulated by subliminal messaging (Karremans, Stroebe, \& Claus, 2006).

The general phenomenon of a facilitative or inhibitory effect on the behavioral response to a stimulus by a previously presented stimulus is what psychology calls priming. In the visual domain, priming can be used to test whether visual discrimination is affected by the nature of prior stimuli. In case both stimuli share the to-be-discriminated feature, they are said to be congruent. Otherwise, they are referred to as incongruent with regard to that particular feature. The estimation of the influence of the prime on the target stimulus is quantified by comparing a behavioral measure, usually reaction times, in response to congruent versus incongruent stimuli. The resultant difference score is referred to as the priming effect.

If the prime is rendered invisible, for example through visual masking, the behavioral response to the target stimulus can tell us in how far the prime is still processed. Priming is therefore an ideal manner of studying the behavioral influence of unconscious stimuli, and subliminal priming has indeed been applied in many psychophysical studies (e.g. Eimer \& Schlaghecken, 2003; Enns \& Di Lollo, 2000; Li, Moallem, Paller, \& Gottfried, 2007; Neumann, Esselmann, \& Klotz, 1993; Vorberg, Mattler, Heinecke, Schmidt, \& Schwarzbach, 2003). Similar to Vicary's claims, these studies demonstrate that a subliminal visual stimulus can modulate later behavior, even in these simplified laboratory priming tasks. Thus, it appears that behavioral modulation occurs independently of visual awareness, indicating that priming might be yet another visual process with a unique neural foundation.

Note that the cases of blindsight, subliminal perception, and subliminal priming do not only bear relevance to researchers of visual perception, but also to philosophers studying the nature of (human) consciousness, as the concepts cast doubt on the functional properties of consciousness. If we can fairly adequately act upon unperceived environmental stimuli, then why do we need conscious experience at all? A thorough explanation of the philosophy of consciousness and an interpretation of the results presented in this thesis in a 
philosophical context go beyond the scope of the thesis. Nevertheless, we would like to point out that our findings apply here as well.
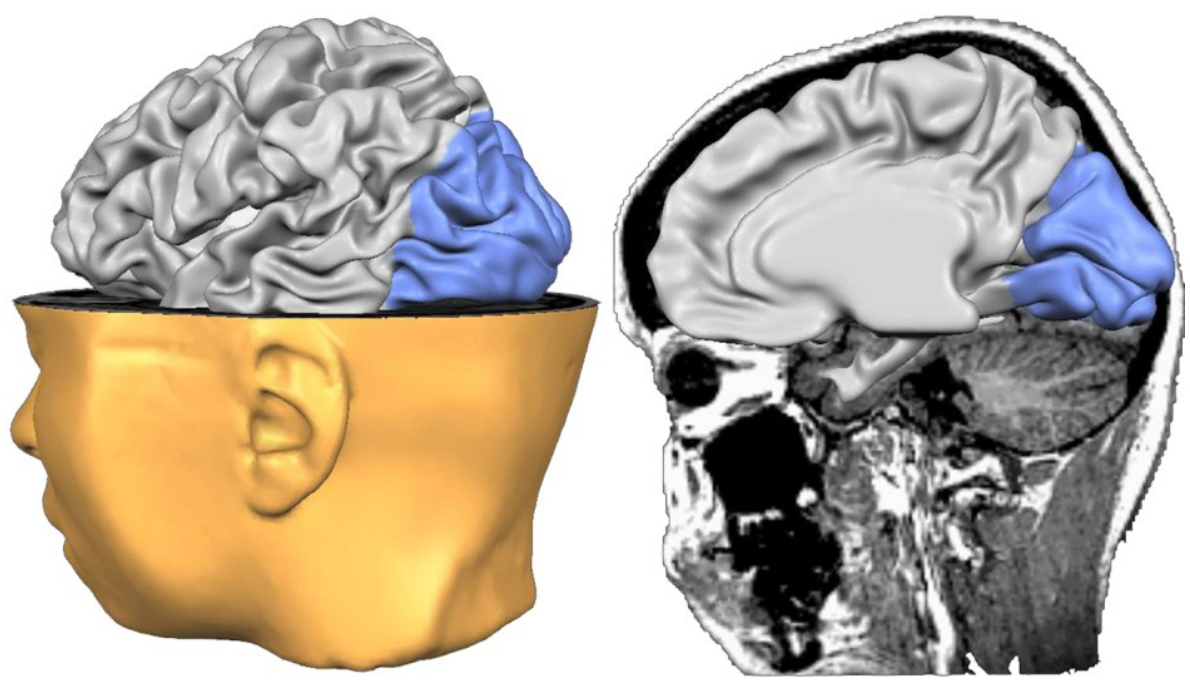

Figure 1. In the occipital lobe (blue), the cortical part of the human visual system is located. Created with BrainTutor (BrainInnovation, Maastricht, the Netherlands)

\section{Visual short-term memory}

Next to visual awareness, visual discrimination and behavioral priming, a final visual process to be introduced here is visual short-term memory (VSTM). Short-term memory is a rather broad concept, but generally it refers to a mental limited-capacity storage, that keeps information in mind for short periods of time. Moreover, any kind of information, that is currently available for cognitive or perceptual operations, is stored in short-term memory, whether it consists of recently acquired information or information which has to be retrieved from long-term memory.

Experimental short-term memory tasks consist of three phases. In the first phase (encoding), the participant is presented with a novel stimulus, which he has been instructed to remember for a short time interval, usually a couple of seconds. Throughout the second phase (consolidation and retention), the participant has to remember the stimulus. Typically, short-term memory tasks are designed in such a way that transfer to long-term memory storage is impossible, and that stimulus information thus has to remain activated in shortterm memory. During the third phase (retrieval), the participant's short-term memory performance is tested, for example by measuring how accurate the participant is, when comparing the stored short-term memory representation to a presented target stimulus. In tasks of VSTM, specifically the visual properties of the stimulus need to be remembered. 
In the case of subliminal priming, visual information is retained and applied to a later stimulus in an implicit way, whereas in tasks of VSTM, there is an explicit demand for stimulus retention. VSTM therefore has more of a cognitive component than behavioral priming, and the neural correlates of short-term memory have been placed in the executive, frontal part of the human brain (Linden, et al., 2003; Mottaghy, Gangitano, Sparing, Krause, \& Pascual-Leone, 2002; Owen, et al., 1998; Todd \& Marois, 2004). Interestingly though, the presentation of a visual mask up to $500 \mathrm{~ms}$ into the retention phase, affects short-term memory performance (Magnussen \& Greenlee, 1999), erasing the retained visual image as it were. The concept of 'memory masking' implies that current visual input can interfere with on-line short-term memory representtations at some point along the visual processing pipe-line. In other words, this psychophysical phenomenon indicates that the part of the human visual brain responsible for stimulus encoding is also employed during consolidation.

In summary, vision has multiple different aspects that are dissociable at the behavioral level, which gives reason to believe that they have different underlying neural mechanisms as well. In the next section, the brain areas implicated in vision and the connectivity between them will be discussed. Special attention is given to the cortical regions that receive the visual information first, because blindsight patients experience damage to these brain areas, and they could therefore be at the heart of the divergence between conscious and unconscious processing.

\section{THE ORGANIZATION OF THE HUMAN VISUAL SYSTEM}

Visual perception is a rich experience, even if it might seem like something so natural to us. In the visual scene depicted in Figure 2, a multitude of colors and forms are present, which make up different objects, that all need to be separated from each other, and from the background. There are people in the image, other natural objects, like trees, but also man-made objects like buildings or cars. The group of protesters in the front immediately draws attention, unlike the trees in the background. And then there is information about the direction of motion of the people in the scene. All these kinds of visual information need to be processed somehow and somewhere. Given that the brain fulfills all these tasks accurately and virtually effortlessly, the need for an intelligently evolved visual system becomes apparent.

In essence, the brain takes a 'divide-and-conquer' approach to the challenge of visual perception. The diverse elements of a visual scene are segregated and processed in particular brain areas, an example being visual motion which is largely processed in the human motion complex (hMT)+ or visual area V5 (see Figure 3). Of course the light that falls on the retina is not directly transmitted to these specialized cortical regions. 


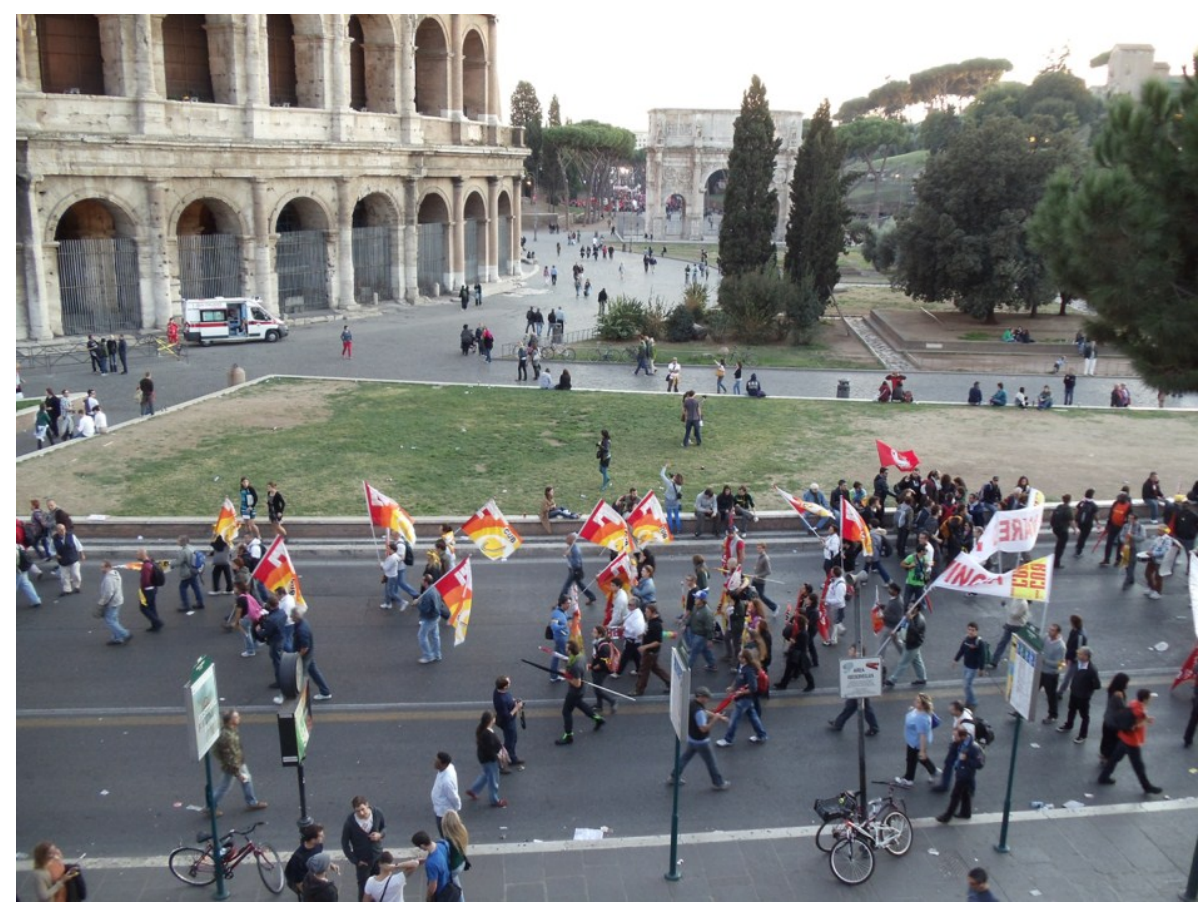

Figure 2. Example of a complex visual scene. Even if the image seems like nothing spectacular, it is quite an ordeal to create a comprehensive visual percept from all the information it contains. There is depth in the image: the people in the front appear closer than the trees and buildings in the background. There is motion in the image: the people in the front appear to be moving in a particular direction rather than to be standing still. And obviously there are all different sorts of colors and shapes in the image that together make up the objects in the picture. The brain has to adequately process all this information and consequently put all the bits of information together to create a unified visual percept.

In the $\sim 100 \mathrm{~ms}$ before the information finally reaches these areas, it has already passed through a number of subcortical and cortical stages. Light is transformed into a neural signal within the retina. The optic nerve then carries this signal to the lateral geniculate nucleus (LGN) of the thalamus. A small number of projections do not lead to LGN, but takes a different subcortical route via the superior colliculi and pulvinar structures. The major pathway continues from LGN to V1, and further to extrastriate areas, i.e. cortical visual areas beyond V1.

The earliest cortical regions of the visual processing stream are characterized by similar properties. Common to visual areas V1, V2 and V3 is the topographic, or retinotopic, representation of the visual field, meaning that adjacent areas in the visual field are processed by cortical neurons which are next to each other. Early visual cortex (EVC), which is the collective term for areas V1, V2 and V3, can be said to contain a photograph of the visual input. The photograph is massively distorted though, because the visual representation is flipped both horizontally and vertically, and it is enlarged in the center of the 
visual field, where vision is the most detailed; a property called cortical magnification. The structure of the cortical pathway is such that successive areas execute processing across a larger area of the visual field, incorporating more information and thus performing increasingly more abstract processing. The pathway is therefore considered hierarchical, and information is said to 'ascend' the visual system (see Figure 3).

In contrast to the hierarchical, or feedforward projections, visual neurons are also connected to areas lower in the visual hierarchy or within the same visual area. These feedback and horizontal connections are referred to as recurrent or re-entrant, and they have been implied in many visual processes, such as figure-ground segregation or perceptual learning (Ahissar \& Hochstein, 2004; Heinen, Jolij, \& Lamme, 2005; Hupe, et al., 1998; Juan \& Walsh, 2003; Koivisto \& Silvanto, 2011; Roelfsema, Lamme, Spekreijse, \& Bosch, 2002; Super, 2002; Super, Spekreijse, \& Lamme, 2003). In addition, theories of visual perception have been proposed that assign great significance to recurrent activity (Bullier, 2001; Hochstein \& Ahissar, 2002; Lamme \& Roelfsema, 2000; Pollen, 1999). In reverse-hierarchy theory (RHT), for example, the role of feedforward activity is the quick transfer of information to downstream visual areas with larger receptive fields, leading to the fast constitution of a global image ('vision at a glance'). Only through feedback activity a more fine-grained image is subsequently created ('vision for scrutiny'). Another purpose ascribed to recurrent activity is the continuous comparison of current input with the visual representation, which is being processed in higher-level visual areas (Di Lollo, Enns, \& Rensink, 2000). Lately, visual awareness has been added to the list of possible functions of recurrent processing. In other words, recurrent processing has been proposed to embody the neural correlate of consciousness (NCC; Edelman, 1992; Lamme \& Roelfsema, 2000; Pollen, 1999; Ro, Breitmeyer, Burton, Singhal, \& Lane, 2003).

Because, in the neurological syndrome of blindsight, damage to V1 causes the abolition of visual awareness, visual awareness and V1 are undoubtedly linked. Proponents of the theory, which assigns the role of NCC to recurrent processing, argue that blindsight occurs because reentry from higher-level visual cortex to V1 is blocked. If they prove to be correct, and recurrent processing is indeed responsible for conscious vision, then what about unconscious vision? There are two possible scenarios: either information of subliminal stimuli is processed by the visual pathway flowing from superior colliculi and pulvinar to extrastriate areas, or the information is processed by LGN and V1, but in a strictly feedforward manner. The occurrence of blindsight obviously speaks in favor of the former option. However, the two alternatives need not be mutually exclusive, and they might in fact both contribute to unconscious visual processing.

Memory masking in short-term memory tasks implies visual cortex contribution during stimulus retention. No analogy with lesion patients can be drawn here, as a lesion in EVC already hampers the earliest stage of short-term memory processing, namely encoding. Therefore, another way of investigating the functional relevance of EVC for VSTM has to be considered. Preferably, it would also show whether the short-term memory representation has a topographical 


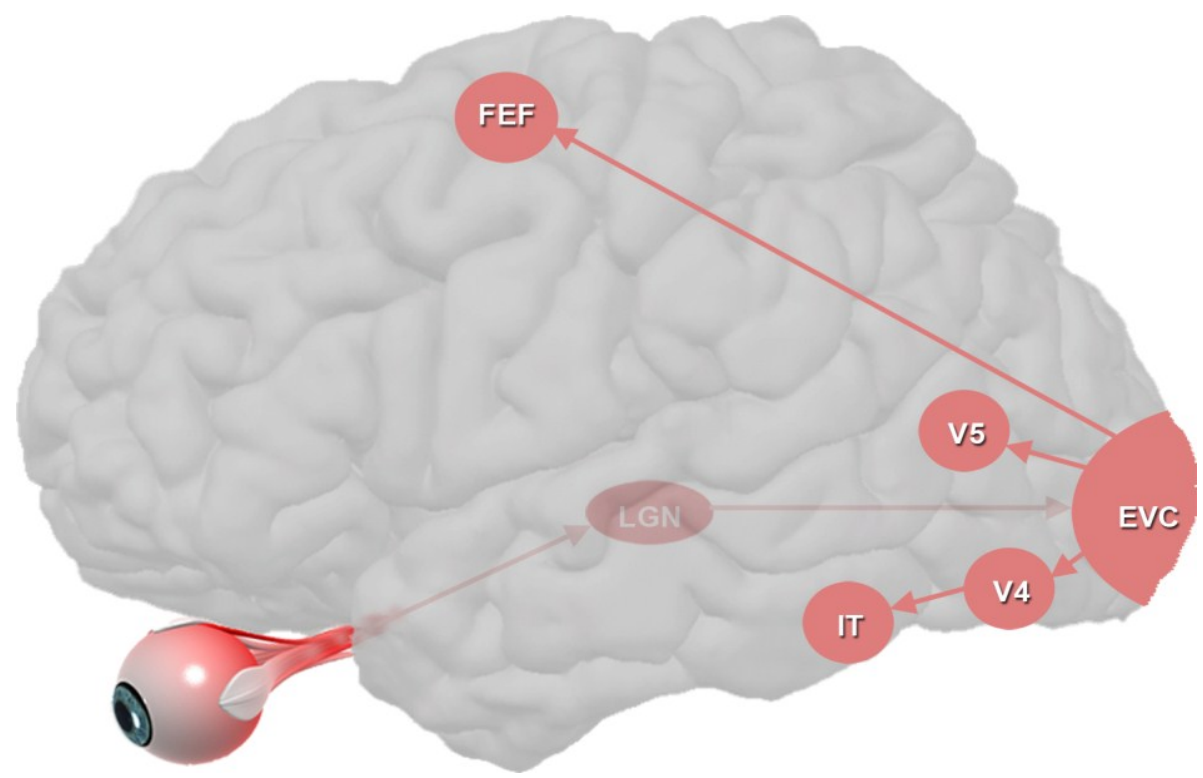

Figure 3. Schematic overview of the human visual hierarchy. Light that falls on the retina is converted into a neural signal, which is subsequently transferred to the lateral geniculate nucleus (LGN) of the thalamus. From there, the signal continues to cortical areas V1, V2 and V3, which are together referred to as early visual cortex (EVC). V4 (color), V5 (motion) and IT (objects) are examples of visual brain areas dedicated to feature processing. Frontal eye fields (FEF) are mainly involved in direction of spatial attention and control of eye movements. Because they become active quite early in the processing stream, they are usually considered early visual areas as well. The visual pathway as presented here is the dominant route. Note, that there is a second pathway via the superior colliculi and pulvinar nucleus of the thalamus, which is not depicted in this figure.

lay-out similar to the original stimulus, indicating that the same neurons responsible for stimulus encoding are also involved in consolidation. Fortunately, TMS offers a way to investigate all these open questions in healthy individuals with a normally functioning visual cortex and under controlled experimental conditions.

\section{TRANSIENT BLINDNESS}

\section{Transcranial Magnetic Stimulation (TMS)}

Since the introduction in 1985 (Barker, Jalinous, \& Freeston, 1985), TMS has developed into a standard and relevant tool to study perception and cognition in the human brain. TMS is a so-called brain interference technique, which refers to a class of neuroscience methods that all share the ability to interfere with the regular processing of the brain. As the name implies, TMS does so by sending a magnetic field into the brain through an intact cranium. 
The TMS stimulator, which is basically a battery of capacitors, sends a very strong alternating current through a copper coil, which is positioned tangential to the skull. Following the fundamental principles of electromagnetic induction, the electrical current within the coil produces a magnetic field in the perpendicular direction. Because a magnetic field can travel through tissue undistorted, it is carried across the skull and penetrates the brain right underneath the surface of the coil; a property which allows TMS to exert its neural effects with certain spatial specificity. The very short duration $(<600 \mu \mathrm{s})$ of the physical TMS pulse, in combination with the short duration of the physiological TMS effect (1-2ms), are the reasons why TMS has an excellent temporal specificity.

The physical properties of TMS bring about its physiological consequences in the following way: within the stimulated region of the brain, the changing magnetic field creates an electric field, which in turn causes depolarization, i.e. a reduction in membrane potential, of the affected neurons. If the amount of depolarization crosses a certain threshold, the neuron will fire, which means that it will send a signal to connected neurons. In essence, TMS thus triggers the normal neuronal communication mechanisms of the brain. However, because it does so to a vast amount of neurons simultaneously, the pattern of neuronal signaling becomes uninformative to other brain regions, and the stimulated region is therefore functionally hampered (Wasserman, et al., 2008).

\section{Advantages of TMS in the study of vision}

Many studies on visual perception have employed TMS. There are a number of advantages which make TMS particularly suited to investigate the workings of the human visual system, and particularly the early visual cortex.

First, TMS can alter brain functioning. This brings the activity of the targeted cortical area under the experimenter's control. The experimental manipulation of brain activity can be linked to consequential changes in behavior, and, in this manner, conclusions about the causal brain-behavior relationship can be drawn. For the visual processes under investigation here, this means that, if EVC is involved in all of them, which is most likely the case, TMS might still be able to tell apart the different functional roles EVC plays in each of them. However, in neuroimaging research, behavior serves as the independent variable, and the covariant brain activity is presumed to play a functional role in the behavior; a presumption that cannot be verified.

Second, TMS has a spatial resolution in the order of centimeters. This is particularly valuable when targeting the early visual cortices, because of their retinotopic organization. The spatial specificity of TMS allows for a topographically specific interference with visual processes. In addition, TMS has a temporal resolution in the order of milliseconds. Hence, besides insight into whether a given brain area functionally contributes to task performance, TMS can also render insight about when that particular brain area is relevant. With regard to visual perception, the role of EVC across multiple visual tasks possibly varies over time. Many TMS studies on visual perception have therefore resorted to chronometric TMS, i.e. the application of TMS across a range of time 
windows, to probe EVC's temporal profile in a given visual task. Given that recurrent cortical loops have been implied in many visual functions, these loops could become apparent in a chronometric TMS study as periodic behavioral impairments.

Third, TMS can induce phosphenes. Phosphenes are phenomenal experiences of visual percepts in the absence of physical light stimulation. Apart from the practical advantage that the position of a phosphene within the visual field can yield information about the corresponding retinal location which is affected by EVC stimulation, phosphenes are themselves a topic worthy of investigation, as they offer an estimate of cortical excitability. The intensity at which occipital TMS induces phosphenes on 50 percent of trials is called the phosphene threshold. The lower the phosphene threshold, the more easily a phosphene can be induced, and thus the more excitable is occipital cortex.

\section{Current state of TMS research on vision}

Since the seminal study of Amassian et al. (1989), who was the first to apply chronometric TMS to the occipital lobe, TMS research on visual perception has taken off. The original findings of this classic study are thus far undisputed, although their interpretation remains a subject of extensive debate. Amassian et al. (1989) found that visual discrimination suffered from a single pulse of occipital TMS, but only when this pulse was applied $\sim 90 \mathrm{~ms}$ after the onset of the visual display. Ample studies have replicated this result since (e.g. Amassian, et al., 1989; Beckers \& Homberg, 1991; Camprodon, Zohary, Brodbeck, \& PascualLeone, 2009; Corthout, Uttl, Juan, Hallett, \& Cowey, 2000; Corthout, Uttl, Walsh, Hallett, \& Cowey, 1999; Corthout, Uttl, Ziemann, Cowey, \& Hallett, 1999; de Graaf, Cornelsen, Jacobs, \& Sack, 2011; Heinen, et al., 2005; Jolij \& Lamme, 2005; Lamme, 2006; Laycock, Crewther, Fitzgerald, \& Crewther, 2007; Overgaard, Nielsen, \& Fuglsang-Frederiksen, 2004; Ro, 2008; Sack, van der Mark, Schuhmann, Schwarzbach, \& Goebel, 2009). There is, however, debate as to whether the $90 \mathrm{~ms}$ post-stimulus TMS time window reflects the feedforward sweep or recurrent activity. Some have found an even earlier time window of TMS-induced masking, and therefore assume that it is feedback activity that TMS interferes with at 90ms (Corthout, Uttl, Walsh, et al., 1999; Corthout, Uttl, Ziemann, et al., 1999; Laycock, et al., 2007; Overgaard, et al., 2004). Others have discovered a later time window, and take the $90 \mathrm{~ms}$ time window to be feedforward activity (Camprodon, et al., 2009; Heinen, et al., 2005). Nonetheless, together these studies have shown that occipital TMS can be used to induce transient scotoma and as such to 'mask' visual stimuli similarly to visual masks in psychophysical research. Hence, the TMS-induced masking approach has been used frequently to disentangle the different roles that EVC plays in a multitude of visual processes ${ }^{1}$.

\footnotetext{
${ }^{1}$ Note, that in many TMS studies phosphene induction is used as a means to localize which coil position on the skull causes stimulation of the topographical target position within the EVC. In the early days, researchers believed that phosphenes always arose from V1, and they therefore inter-
} 
First, consider the dissociation between visual discrimination and visual awareness. Extending the classic paradigm of a visual discrimination task combined with chronometric TMS, a second dependent variable in the form of selfrated awareness has been added to learn about the concurrent conscious experience on a trial-by-trial basis. From here, different approaches have been taken to utilize this information in an attempt to dissociate visual awareness and discrimination in EVC. The first procedure is to simply plot the functional relevance of EVC for both processes over time, and to search time windows at which TMS selectively interferes with visual awareness, but not with discrimination performance (de Graaf, Herring, \& Sack, 2011; Koivisto, Railo, \& Salminen-Vaparanta, 2011). Recurrent theories about visual awareness would for instance predict that there are at least two crucial time windows at which TMS affects visual awareness, whereas there is only one at which discrimination performance suffers. As mentioned earlier, until now multiple time windows of visual suppression by TMS have been reported, although the $\sim 90 \mathrm{~ms}$ time window is the only time window that consistently emerges from the literature on TMS masking. Moreover, many of the studies that found additional time windows did not incorporate a subjective measure of awareness (Camprodon, et al., 2009; Corthout, et al., 2000; Corthout, Uttl, Walsh, et al., 1999; Corthout, Uttl, Ziemann, et al., 1999; Overgaard, et al., 2004), making it impossible to draw the conclusion that any TMS effect is specific to conscious perception.

A different way of approaching the matter is to try and induce blindsight in healthy individuals with TMS. In that case, accuracy scores are calculated for the TMS trials on which subjective awareness was absent, and statistically tested against chance level performance. If performance proves to exceed chance level, this would reflect 'TMS-induced blindsight'. This approach has proven to be more successful (Boyer, Harrison, \& Ro, 2005; Christensen, Kristiansen, Rowe, \& Nielsen, 2008; Jolij \& Lamme, 2005; Ro, Shelton, Lee, \& Chang, 2004), and TMS-induced as well as clinical blindsight have been interpreted as support for a subcortical pathway of unconscious vision that circumvents EVC.

A last approach, specifically suitable to examine whether feedback to EVC is essential to conscious perception, is the comparison of the temporal involvement of multiple connected regions of visual cortex. Pascual-Leone and Walsh (2000) stimulated human visual motion area V5/hMT+ with single pulses of TMS, each of which induces visual awareness in the shape of moving phosphenes. A second (sub threshold) TMS pulse was delivered over V1 to suppress the visual information processing in that area. The temporal delay between these two pulses was variable. Based on recurrent models of visual awareness one would predict that the TMS-induced perceptual motion activity in V5 can only reach conscious awareness after the information has been backprojected to V1. The authors indeed found that the phosphene-related percept was impoverished if a TMS pulse was applied to V1 5-45ms after V5 stimulation. Later,

preted their findings to relate to the role of V1 in vision. Today, we recognize that phosphenes most probably originate from V2 and the dorsal part of V3 (Thielscher, Reichenbach, Ugurbil, \& Uludag, 2009), and that it is very difficult to selectively stimulate V1 due to its anatomical location within the calcarine sulcus (Salminen-Vaparanta, Noreika, Revonsuo, Koivisto, \& Vanni, 2011). Therefore, we choose to refer to early visual cortex instead. 
this result was replicated with real motion stimuli (Silvanto, Lavie, \& Walsh, 2005).

Analogous to behavioral masked priming studies, masking by occipital TMS might be used to investigate the behavioral impact of unconscious visual stimuli. Moreover, TMS masking can be applied to investigate whether subliminal stimuli need processing by EVC in order to influence future behavior. Possibly, the unconscious prime-related processing circumvents EVC altogether, and takes the subcortical route via superior colliculi and pulvinar instead. Using such a TMS-induced masked priming set-up, Sack et al. (2009) discovered that in fact intact EVC is required for behavioral priming. Both priming and visual discrimination were affected by occipital TMS at the same time windows, namely the classic 80 and $100 \mathrm{~ms}$ post-stimulus onset, leaving unanswered the question of what dissociates the two processes.

The classic $\sim 90 \mathrm{~ms}$ time window of TMS masking appears to be central to visual awareness, visual discrimination and behavioral priming. Other poststimulus time windows have been reported, though none of them has been repeatedly established thus far. In the pre-stimulus domain, visual suppression has been demonstrated recurrently, although, yet again, the interpretation of the effect has been contested (Beckers \& Homberg, 1991; Corthout, et al., 2000; de Graaf, Cornelsen, et al., 2011; Laycock, et al., 2007; Sack, Kohler, Linden, Goebel, \& Muckli, 2006; Stevens, McGraw, Ledgeway, \& Schluppeck, 2009). Keeping the physiological workings of TMS in mind, it seems rather counterintuitive that a TMS pulse preceding the visual stimulus can reduce its visibility. After all, there is no visual signal in EVC yet for the TMS pulse to suppress. The prestimulus TMS masking effect has consequently been attributed to eye blinking (Beckers \& Homberg, 1991; Corthout, et al., 2000; Sack, et al., 2006), which is conceptually far more intuitive, because an eye blink would block the visual input at the much earlier, retinal stage. The recent discovery that pre-stimulus waves of neuronal activity (with a frequency of $\sim 10 \mathrm{~Hz}$ ) can predict stimulus visibility could inspire a reconsideration of the eye blink account of pre-stimulus TMS masking (Dugue, Marque, \& VanRullen, 2011; Hanslmayr, et al., 2007; Mathewson, Gratton, Fabiani, Beck, \& Ro, 2009; Romei, et al., 2008; van Dijk, Schoffelen, Oostenveld, \& Jensen, 2008). Assume that pre-stimulus TMS brings about an unreceptive neural state, and EVC is still lingering in this state when the stimulus-related input from LGN enters EVC. Then the pre-stimulus TMS effect would in fact have a neural origin, even if it does not directly obstruct visual processing. If pre-stimulus TMS masking is indeed of neural nature, it would be sensible to broaden our search for the correlates of visual awareness, visual discrimination and priming to the pre-stimulus domain.

TMS studies probing the functional involvement of visual cortex in VSTM rendered mixed results, ranging from no effect during short-term memory retention (Beckers \& Homberg, 1991), to differential effects dependent on the temporal position of the occipital TMS pulse in the retention interval (Cattaneo, Vecchi, Pascual-Leone, \& Silvanto, 2009). VSTM has also been linked to conscious visual perception. Silvanto \& Cattaneo (2010) showed that motion perception of phosphenes elicited over V5/hMT+ was enhanced when the motion direction was identical to that of a visual stimulus currently stored in 
VSTM. However, this was only the case when the spatial location of the memory trace and induced phosphene overlapped. These results were interpreted as identical neuronal populations being involved in VSTM retention and conscious perception.

In summary, occipital TMS has proven to be a valuable instrument to unravel the role EVC plays in a number of (related) visual processes, even if not all questions have been given a definite answer yet. In the next section, the idea of combining TMS with brain imaging methods is introduced. In the context of chronometric TMS and the visual system, the combination of TMS and EEG is of great value.

\section{Combining TMS with other neuroscience techniques}

As the research overview nicely demonstrates, TMS is a powerful research tool to study the visual system. However, like any scientific research method, TMS is limited with regard to the research questions it could answer. In classic TMS experiments, the dependent variable is always some kind of behavioral measure, be it muscular reactions, button presses or verbal reports. Suppose a TMS pulse actually affects the stimulated target area, but simply too weak to cause a behavioral impairment, for example because the task is too easy. Then, the suppressive effect of TMS will not show in the behavioral data. This is a fundamental problem to TMS research, and one of the reasons why null results in TMS research are especially hard to interpret. Instead of measuring behavior, which is the final output of the most complex of systems and which might not be sensitive to disruption at one of its hubs, an alternative would be to actually look into the brain in order to see whether changes occur at a neural level due to TMS.

Moreover, even if a TMS pulse exerts a measurable influence on the dependent variable, the effects need not stem merely from the targeted brain area. Perceptual and cognitive functions are performed by intricate networks of brain regions, consisting of multiple densely connected neural nodes. Disabling a node within such an integrated network can affect multiple brain regions, because the TMS effects may spread to distant nodes within the functional network, connected with the site of TMS stimulation. Perhaps the network is even flexible enough to counteract the effect of the TMS pulse, as a different node might take over the role of the stimulated area. Again, to map these remote or compensatory effects, the concurrent acquisition of behavioral and neural measures in TMS research is required.

Most commonly, TMS is used in combination with either functional magnetic resonance imaging (fMRI) or electroencephalography (EEG), the two most widespread imaging methods in human neuroscience, to map the exact spatial network effects of TMS or chart the neural influences of TMS across time, respectively. Besides the advantages of these kinds of multimodal research, there are limitations that need to be overcome, some of which are inherent to the used technique and therefore principally unsolvable, and others which can be solved through appropriate data pre-processing. 
EEG is a neurophysiological method that measures the tiny electrical currents generated by neuronal populations at the surface of the brain through sensitive electrodes which are positioned on the skull. The recording of this signal happens almost instantaneously, and EEG is therefore suitable to investigate the development of the neural TMS effect over time with very high precision. As mentioned before, most TMS research on vision has focused on the functional relevance of EVC over time, and concurrent EEG acquisition is therefore a logical next step. Whereas EEG has superb temporal resolution, the conductive properties inherent to the different tissue types within the brain, skull, and scalp distort the electrical signal leading to compromised spatial resolution.

Given the electrical properties of the signal measured with EEG, combining the method with TMS is far from trivial. A small amount of data has to be sacrificed following the TMS pulse, either by temporarily halting data acquisition, or simply by disregarding the TMS-distorted samples. The duration of the EEG distortion is a quintessential parameter in simultaneous TMS-EEG experiments on visual perception, because many studies within this field employ TMS chronometrically, leaving only tenths of milliseconds in between occipital TMS pulse and visual stimulus. The electrical TMS artifact in the EEG-signal due to induced currents in the surface electrodes has subsided in a little less than $\sim 6 \mathrm{~ms}$, and a second minor artifact due to the recharging of the TMS stimulator occurs within 70ms after pulse-onset (Veniero, Bortoletto, \& Miniussi, 2009).

Besides the immediate pulse artifact, there are other ways in which TMS affects the EEG. TMS-locked physiological noise can distort the EEG signal beyond the recharge artifact, up until 200ms post pulse-onset (Ilmoniemi \& Kicic, 2010). Cleaning the EEG data of these longer-lasting TMS contaminations is a sensitive matter, because there is a severe risk of removing the neurophysiological effect-of-interest in the process.

Nevertheless, chronometric TMS-EEG studies on vision have been successfully performed (e.g. Reichenbach, Whittingstall, \& Thielscher, 2011; Thut, et al., 2003). In a post-stimulus TMS masking paradigm, the neurophysiological responses to visual stimulation as measured with EEG were found to be enhanced by occipital TMS (Reichenbach, et al., 2011). The TMS-induced enhancement increased with increasing stimulation intensities, but saturated when task performance started to break down. This finding is rather surprising, because the functional impairment in TMS masking was previously linked to neural signal suppression (Harris, Clifford, \& Miniussi, 2008). Reichenbach et al. (2011) interpreted their data as a reflection of the robustness of the neural visual code to heightened noise levels, as inflicted with TMS in their experiment. The study is a perfect example of the merit of combined electrophysiological and behavioral data acquisition in TMS paradigms, because the inverse relation between neural and behavioral responses could only have been established via a multimodal imaging approach. 


\section{THESIS OUTLINE}

The research conducted within the scope of this thesis aimed to investigate the role of EVC in multiple aspects of visual perception. The functional relevance of EVC was assessed by perturbing it with TMS during execution of various visual tasks.

The neuropsychological syndrome of blindsight is characterized by a dissociation between visual awareness and task performance on a range of visual tasks. This phenomenon formed the inspiration for Chapter 2. In this chapter, the dependence of visual awareness and visual discrimination on EVC was assessed through occipital TMS across a broad range of time windows, ranging from $-80 \mathrm{~ms}$ pre-stimulus to $300 \mathrm{~ms}$ post-stimulus. Appropriate control conditions were included to verify that the revealed TMS effects are not due to nonneural influences of TMS. The inclusion of these control conditions allowed, for the first time, a systematic investigation of the (neural) nature of pre-stimulus TMS masking.

In Chapter 3, the behavioral influence of a visual stimulus on later behavior, in other words its priming capacity, and the role of EVC therein was explored. On the basis of theories implying feedback processing in conscious vision and the notion that priming can occur in the absence of conscious vision, we expected an additional time window of EVC relevance for visual awareness, compared to behavioral priming. To test this hypothesis, TMS-induced suppression of self-reported awareness, visual discrimination and priming were measured in identical experimental contexts.

Chapter 4 examines whether EVC processing is also essential for a cognitive operation performed on visual information, namely short-term memory. Topographic TMS was delivered at three different timings into the retention interval of a modified change detection task with two different memory loads. The design allowed conclusions about the temporal and spatial specificity, and load dependence of the TMS effect. In a second experiment, a visual 'memory mask' replaced the TMS pulse, and the two methods of memory masking could subsequently be compared.

In order to investigate the underlying physiology of pre- and post-stimulus TMS masking, the simultaneous acquisition of electrophysiological data is required. In Chapter 5, two TMS systems are tested on their suitability for concurrent TMS-EEG experiments. In the second section of this chapter, evoked responses to visual stimuli, which were preceded by an occipital TMS pulse, were reconstructed from the EEG signal, and their temporal profile was compared to that on trials without TMS.

Finally, Chapter 6 presents an overview of the main findings mentioned in the empirical chapters of the thesis. The meaning of these findings is subsequently evaluated and discussed in the light of current theoretical frameworks. In conclusion, suggestions are made about the direction that future research on the functional role of EVC across the visual domain could take. 


\section{REFERENCES}

Ahissar, M., \& Hochstein, S. (2004). The reverse hierarchy theory of visual perceptual learning. Trends Cogn Sci, 8(10), 457-464.

Amassian, V. E., Cracco, R. Q., Maccabee, P. J., Cracco, J. B., Rudell, A., \& Eberle, L. (1989). Suppression of visual perception by magnetic coil stimulation of human occipital cortex. Electroencephalogr Clin Neurophysiol, 74(6), 458-462.

Barker, A. T., Jalinous, R., \& Freeston, I. L. (1985). Non-invasive magnetic stimulation of human motor cortex. Lancet, 1(8437), 1106-1107.

Beckers, G., \& Homberg, V. (1991). Impairment of visual perception and visual short term memory scanning by transcranial magnetic stimulation of occipital cortex. Exp Brain Res, 87(2), 421432.

Boyer, J. L., Harrison, S., \& Ro, T. (2005). Unconscious processing of orientation and color without primary visual cortex. Proc Natl Acad Sci US A, 102(46), 16875-16879.

Bullier, J. (2001). Feedback connections and conscious vision. Trends Cogn Sci, 5(9), 369-370.

Camprodon, J. A., Zohary, E., Brodbeck, V., \& Pascual-Leone, A. (2009). Two phases of V1 activity for visual recognition of natural images. J Cogn Neurosci, 22(6), 1262-1269.

Cattaneo, Z., Vecchi, T., Pascual-Leone, A., \& Silvanto, J. (2009). Contrasting early visual cortical activation states causally involved in visual imagery and short-term memory. Eur J Neurosci, 30(7), 1393-1400.

Christensen, M. S., Kristiansen, L., Rowe, J. B., \& Nielsen, J. B. (2008). Action-blindsight in healthy subjects after transcranial magnetic stimulation. Proc Natl Acad Sci U S A, 105(4), 1353-1357.

Corthout, E., Uttl, B., Juan, C. H., Hallett, M., \& Cowey, A. (2000). Suppression of vision by transcranial magnetic stimulation: a third mechanism. Neuroreport, 11(11), 2345-2349.

Corthout, E., Uttl, B., Walsh, V., Hallett, M., \& Cowey, A. (1999). Timing of activity in early visual cortex as revealed by transcranial magnetic stimulation. Neuroreport, 10(12), 2631-2634.

Corthout, E., Uttl, B., Ziemann, U., Cowey, A., \& Hallett, M. (1999). Two periods of processing in the (circum)striate visual cortex as revealed by transcranial magnetic stimulation. Neuropsychologia, 37(2), 137-145.

de Graaf, T. A., Cornelsen, S., Jacobs, C., \& Sack, A. T. (2011). TMS effects on subjective and objective measures of vision: Stimulation intensity and pre- versus post-stimulus masking. Conscious Cogn. 20[4], 1244-1255.

de Graaf, T. A., Herring, J., \& Sack, A. T. (2011). A chronometric exploration of high-resolution 'sensitive TMS masking' effects on subjective and objective measures of vision. Exp Brain Res, 209(1), 19-27.

Di Lollo, V., Enns, J. T., \& Rensink, R. A. (2000). Competition for consciousness among visual events: the psychophysics of reentrant visual processes. J Exp Psychol Gen, 129(4), 481-507.

Dugue, L., Marque, P., \& VanRullen, R. (2011). The phase of ongoing oscillations mediates the causal relation between brain excitation and visual perception. J Neurosci, 31(33), 11889-11893.

Edelman, G.M. (1992) Bright Air, Brilliant Fire. On the Matter of Mind, Basic Books, USA

Eimer, M., \& Schlaghecken, F. (2003). Response facilitation and inhibition in subliminal priming. Biol Psychol, 64(1-2), 7-26.

Enns, J. T., \& Di Lollo, V. (2000). What's new in visual masking? Trends Cogn Sci, 4(9), 345-352.

Hanslmayr, S., Aslan, A., Staudigl, T., Klimesch, W., Herrmann, C. S., \& Bauml, K. H. (2007). Prestimulus oscillations predict visual perception performance between and within subjects. Neuroimage., 37(4), 1465-1473.

Harris, J. A., Clifford, C. W., \& Miniussi, C. (2008). The functional effect of transcranial magnetic stimulation: signal suppression or neural noise generation? J Cogn Neurosci, 20(4), 734-740.

Heinen, K., Jolij, J., \& Lamme, V. A. (2005). Figure-ground segregation requires two distinct periods of activity in V1: a transcranial magnetic stimulation study. Neuroreport, 16(13), 1483-1487.

Hochstein, S., \& Ahissar, M. (2002). View from the top: hierarchies and reverse hierarchies in the visual system. Neuron, 36(5), 791-804.

Hupe, J. M., James, A. C., Payne, B. R., Lomber, S. G., Girard, P., \& Bullier, J. (1998). Cortical feedback improves discrimination between figure and background by V1, V2 and V3 neurons. Nature, 394(6695), 784-787.

Ilmoniemi, R. J., \& Kicic, D. (2010). Methodology for combined TMS and EEG. Brain Topogr, 22(4), 233-248 
Jolij, J., \& Lamme, V. A. (2005). Repression of unconscious information by conscious processing: evidence from affective blindsight induced by transcranial magnetic stimulation. Proc Natl Acad Sci U S A, 102(30), 10747-10751.

Juan, C. H., \& Walsh, V. (2003). Feedback to V1: a reverse hierarchy in vision. Exp Brain Res, 150(2), 259-263.

Karremans, J. C., Stroebe, W., \& Claus, J. (2006). Beyond Vicary's fantasies: The impact of subliminal priming and brand choice. Journal of Experimental Social Psychology, 42(6), 792-798.

Koivisto, M., Railo, H., \& Salminen-Vaparanta, N. (2011). Transcranial magnetic stimulation of early visual cortex interferes with subjective visual awareness and objective forced-choice performance. Conscious Cogn, 20(2), 288-298.

Koivisto, M., \& Silvanto, J. (2011). Relationship between visual binding, reentry and awareness. Conscious Cogn. 20[4], 1293-1303.

Lamme, V. A. (2006). Zap! Magnetic tricks on conscious and unconscious vision. Trends Cogn Sci, 10(5), 193-195.

Lamme, V. A., \& Roelfsema, P. R. (2000). The distinct modes of vision offered by feedforward and recurrent processing. Trends Neurosci, 23(11), 571-579.

Lau, H. C., \& Passingham, R. E. (2006). Relative blindsight in normal observers and the neural correlate of visual consciousness. Proc Natl Acad Sci U S A, 103(49), 18763-18768.

Laycock, R., Crewther, D. P., Fitzgerald, P. B., \& Crewther, S. G. (2007). Evidence for fast signals and later processing in human V1/V2 and V5/MT+: A TMS study of motion perception. $J$ Neurophysiol, 98(3), 1253-1262.

Li, W., Moallem, I., Paller, K. A., \& Gottfried, J. A. (2007). Subliminal smells can guide social preferences. Psychol Sci, 18(12), 1044-1049.

Linden, D. E., Bittner, R. A., Muckli, L., Waltz, J. A., Kriegeskorte, N., Goebel, R., et al. (2003). Cortical capacity constraints for visual working memory: dissociation of fMRI load effects in a frontoparietal network. Neuroimage, 20(3), 1518-1530.

Magnussen, S., \& Greenlee, M. W. (1999). The psychophysics of perceptual memory. Psychol Res, 62(2-3), 81-92.

Mathewson, K. E., Gratton, G., Fabiani, M., Beck, D. M., \& Ro, T. (2009). To see or not to see: prestimulus alpha phase predicts visual awareness. J Neurosci, 29(9), 2725-2732.

Maurer, D., Lewis, T. L., \& Mondloch, C. J. (2005). Missing sights: consequences for visual cognitive development. Trends Cogn Sci, 9(3), 144-151.

Merabet, L. B., Rizzo, J. F., Amedi, A., Somers, D. C., \& Pascual-Leone, A. (2005). What blindness can tell us about seeing again: merging neuroplasticity and neuroprostheses. Nat Rev Neurosci, 6(1), 71-77.

Mottaghy, F. M., Gangitano, M., Sparing, R., Krause, B. J., \& Pascual-Leone, A. (2002). Segregation of areas related to visual working memory in the prefrontal cortex revealed by rTMS. Cereb Cortex, 12(4), 369-375.

Neumann, O., Esselmann, U., \& Klotz, W. (1993). Differential effects of visual-spatial attention on response latency and temporal-order judgment. Psychol Res, 56(1), 26-34.

Ostrovsky, Y., Andalman, A., \& Sinha, P. (2006). Vision following extended congenital blindness. Psychol Sci, 17(12), 1009-1014.

Overgaard, M., Nielsen, J. F., \& Fuglsang-Frederiksen, A. (2004). A TMS study of the ventral projections from V1 with implications for the finding of neural correlates of consciousness. Brain Cogn, 54(1), 58-64.

Owen, A. M., Stern, C. E., Look, R. B., Tracey, I., Rosen, B. R., \& Petrides, M. (1998). Functional organization of spatial and nonspatial working memory processing within the human lateral frontal cortex. Proc Natl Acad Sci U S A, 95(13), 7721-7726.

Pascual-Leone, A., \& Walsh, V. (2001). Fast backprojections from the motion to the primary visual area necessary for visual awareness. Science, 292(5516), 510-512.

Pollen, D. A. (1999). On the neural correlates of visual perception. Cereb Cortex, 9(1), 4-19.

Reichenbach, A., Whittingstall, K., \& Thielscher, A. (2011). Effects of transcranial magnetic stimulation on visual evoked potentials in a visual suppression task. Neuroimage, 54(2), 13751384.

Ro, T. (2008). Unconscious vision in action. Neuropsychologia, 46(1), 379-383.

Ro, T., Breitmeyer, B., Burton, P., Singhal, N. S., \& Lane, D. (2003). Feedback contributions to visual awareness in human occipital cortex. Curr Biol, 13(12), 1038-1041. 
Ro, T., Shelton, D., Lee, O. L., \& Chang, E. (2004). Extrageniculate mediation of unconscious vision in transcranial magnetic stimulation-induced blindsight. Proc Natl Acad Sci U S A, 101(26), 99339935.

Roelfsema, P. R., Lamme, V. A., Spekreijse, H., \& Bosch, H. (2002). Figure-ground segregation in a recurrent network architecture. J Cogn Neurosci, 14(4), 525-537.

Romei, V., Brodbeck, V., Michel, C., Amedi, A., Pascual-Leone, A., \& Thut, G. (2008). Spontaneous fluctuations in posterior alpha-band EEG activity reflect variability in excitability of human visual areas. Cereb Cortex, 18(9), 2010-2018.

Sack, A. T., Kohler, A., Linden, D. E., Goebel, R., \& Muckli, L. (2006). The temporal characteristics of motion processing in hMT/V5+: combining fMRI and neuronavigated TMS. Neuroimage, 29(4), 1326-1335.

Sack, A. T., van der Mark, S., Schuhmann, T., Schwarzbach, J., \& Goebel, R. (2009). Symbolic action priming relies on intact neural transmission along the retino-geniculo-striate pathway. Neuroimage, 44(1), 284-293.

Salminen-Vaparanta, N., Noreika, V., Revonsuo, A., Koivisto, M., \& Vanni, S. (2011). Is selective primary visual cortex stimulation achievable with TMS? Hum Brain Mapp. 33[3], 652-665.

Silvanto, J., \& Cattaneo, Z. (2010). Transcranial magnetic stimulation reveals the content of visual short-term memory in the visual cortex. Neuroimage, 50(4), 1683-1689.

Silvanto, J., Lavie, N., \& Walsh, V. (2005). Double dissociation of V1 and V5/MT activity in visual awareness. Cereb Cortex, 15(11), 1736-1741.

Stevens, L. K., McGraw, P. V., Ledgeway, T., \& Schluppeck, D. (2009). Temporal characteristics of global motion processing revealed by transcranial magnetic stimulation. Eur J Neurosci, 30(12), 2415-2426.

Super, H. (2002). Cognitive processing in the primary visual cortex: from perception to memory. Rev Neurosci, 13(4), 287-298.

Super, H., Spekreijse, H., \& Lamme, V. A. (2003). Figure-ground activity in primary visual cortex (V1) of the monkey matches the speed of behavioral response. Neurosci Lett, 344(2), 75-78.

Tehovnik, E. J., Slocum, W. M., Smirnakis, S. M., \& Tolias, A. S. (2009). Microstimulation of visual cortex to restore vision. Prog Brain Res, 175, 347-375.

Thielscher, A., Reichenbach, A., Ugurbil, K., \& Uludag, K. (2009). The cortical site of visual suppression by transcranial magnetic stimulation. Cereb Cortex, 20(2), 328-338.

Thomas, S. (2011). Project Prakash: Challenging the critical period: association of research in vision and ophthalmology national meeting. Yale J Biol Med, 84(4), 483-485.

Thut, G., Northoff, G., Ives, J. R., Kamitani, Y., Pfennig, A., Kampmann, F., et al. (2003). Effects of single-pulse transcranial magnetic stimulation (TMS) on functional brain activity: a combined event-related TMS and evoked potential study. Clin Neurophysiol, 114(11), 2071-2080.

Todd, J. J., \& Marois, R. (2004). Capacity limit of visual short-term memory in human posterior parietal cortex. Nature, 428(6984), 751-754.

van Dijk, H., Schoffelen, J. M., Oostenveld, R., \& Jensen, O. (2008). Prestimulus oscillatory activity in the alpha band predicts visual discrimination ability. J Neurosci., 28(8), 1816-1823.

Veniero, D., Bortoletto, M., \& Miniussi, C. (2009). TMS-EEG co-registration: on TMS-induced artifact. Clin Neurophysiol, 120(7), 1392-1399.

Vorberg, D., Mattler, U., Heinecke, A., Schmidt, T., \& Schwarzbach, J. (2003). Different time courses for visual perception and action priming. Proc Natl Acad Sci U S A, 100(10), 6275-6280.

Wasserman, E. M., Epstein, C. M., Ziemann, U., Walsh, V., Paus, T., \& Lisanby, S. H. (Eds.). (2008). The Oxford Handbook of Transcranial Stimulation (First ed.). Oxford: Oxford University Press.

Weiskrantz, L. (2009). Blindsight: a case study spanning 35 years and new developments (3rd ed.). Oxford Oxford University Press. 


\section{Chapter 2 Visual awareness suppression by pre-stimulus brain stimulation; \\ a neural effect}
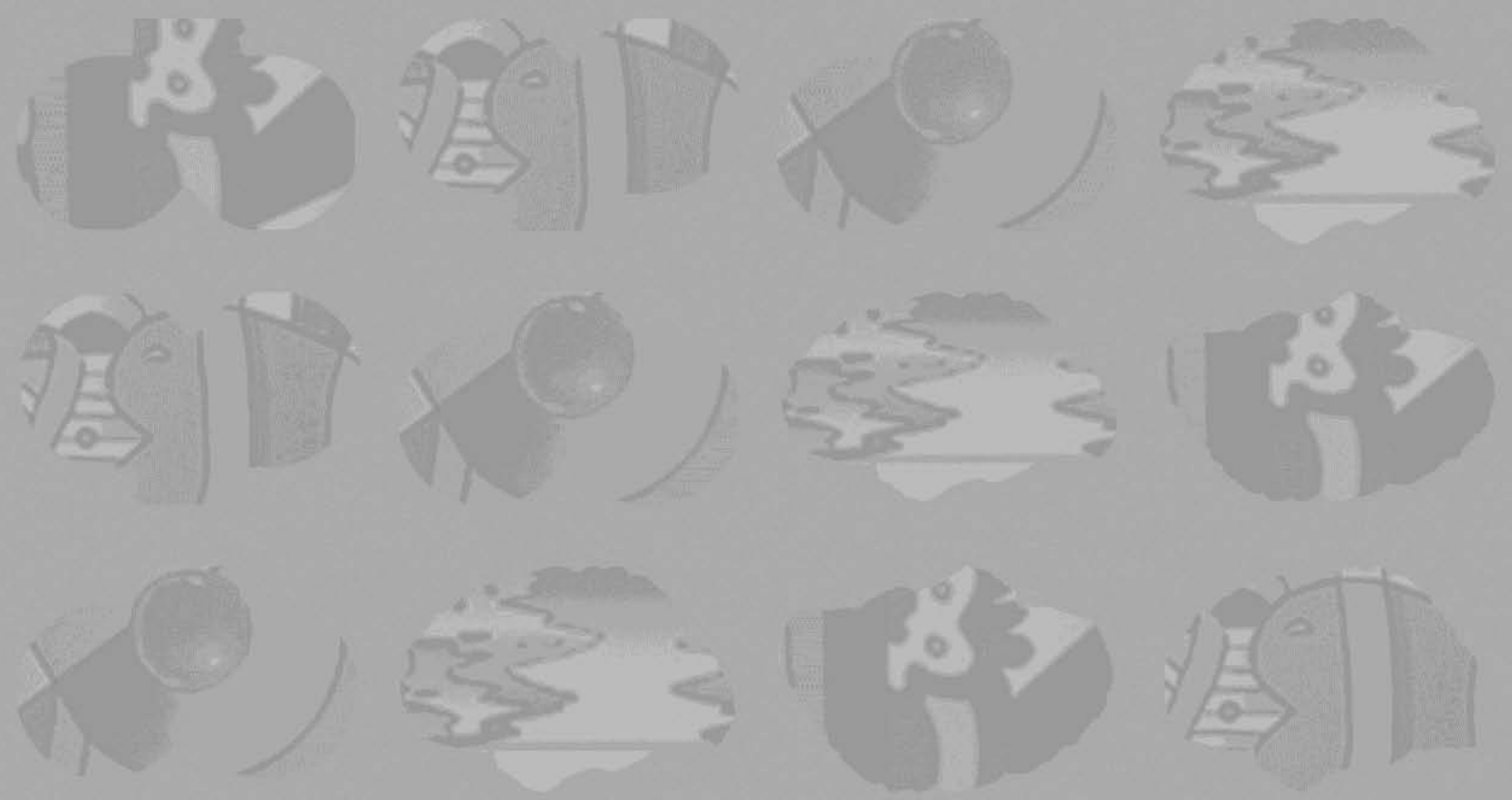

Based on: Jacobs, C., Goebel, R \& Sack, A.T. (2012). Visual awareness suppression by pre-stimulus brain stimulation; a neural effect. Neuroimage, 59(1), 616-24 


\section{ABSTRACT}

Transcranial magnetic stimulation (TMS) has established the functional relevance of early visual cortex (EVC) for visual awareness with great temporal specificity, non-invasively in conscious human volunteers. Many studies have found a suppressive effect when TMS was applied over EVC 80-100ms after the onset of the visual stimulus (post-stimulus TMS time window). Yet, few studies found task performance to also suffer when TMS was applied even before visual stimulus presentation (pre-stimulus TMS time window). This pre-stimulus TMS effect, however, remains controversially debated and its origin had mainly been ascribed to TMS-induced eye blinking artifacts. Here, we applied chronometric TMS over EVC during the execution of a visual discrimination task, covering an exhaustive range of visual stimulus-locked TMS time windows ranging from -80 pre-stimulus to $300 \mathrm{~ms}$ post-stimulus onset. Electrooculographical (EoG) recordings, sham TMS stimulation, and vertex TMS stimulation controlled for different types of non-neural TMS effects. Our findings clearly reveal TMS-induced masking effects for both pre- and post-stimulus time windows, and for both objective visual discrimination performance and subjective visibility. Importantly, all effects proved to be still present after post hoc removal of eye blink trials, suggesting a neural origin for the pre-stimulus TMS suppression effect on visual awareness. We speculate based on our data that TMS exerts its prestimulus effect via generation of a neural state which interacts with subsequent visual input. 


\section{INTRODUCTION}

The neural origin of consciousness is one of the most fundamental and controversially debated topics in cognitive neuroscience at present. Within the domain of vision, much research has centered on the relevance of early visual cortex (EVC), i.e. V1, V2 and V3, for visual awareness, which is still a matter of current debate (Tong, 2003). Several researchers believe EVC to be nothing more than a relay station, passing on the visual information to higher level brain areas (Crick \& Koch, 1995; Zeki \& Bartels, 1998), which they consider the actual loci of visual awareness. However, activity in EVC varies with the nature of the visual percept, as has been shown in fMRI studies on binocular rivalry (Haynes \& Rees, 2005) and apparent motion (Muckli, Kohler, Kriegeskorte, \& Singer, 2005). From these findings, EVC indeed appears to be involved in the initiation of visual awareness. However, correlational measures of brain activity, like fMRI, can never lead to conclusive evidence on matters of functional relevance, as such techniques passively measure brain activity instead of actively manipulating it.

Transcranial magnetic stimulation (TMS) allows the transient disruption of regular neural processing with great temporal as well as spatial resolution. By inducing such a 'virtual lesion' in healthy humans and subsequently investigating behavioral performance, a causal relationship between the stimulated brain area and a cognitive or perceptual faculty can be established; an approach that has already been successfully employed in the study of visual awareness. In this way, an ample number of studies showed that a TMS pulse delivered to EVC roughly 80-100ms post-stimulus onset can effectively impair performance on visual tasks (e.g. Amassian, et al., 1989; Beckers \& Homberg, 1991; Beckers \& Zeki, 1995; Christensen, Kristiansen, Rowe, \& Nielsen, 2008; Corthout, Uttl, Walsh, Hallett, \& Cowey, 1999; Corthout, Uttl, Ziemann, Cowey, \& Hallett, 1999; Sack, van der Mark, Schuhmann, Schwarzbach, \& Goebel, 2009), which is in accord with retinocortical transmission times. Thus, through TMS it was established that in normal vision EVC has a role to play in the generation of visual awareness, be it as the locus of visual awareness or by relaying the information to ascending 'awareness' regions. Moreover, TMS has established that EVC's role in visual awareness is restricted to a certain temporal window at which unperturbed neural activity within EVC is critical for visual awareness.

A remarkable finding was the discovery of an additional time window, when TMS applied prior to the onset of the visual stimulus caused a perceptual impairment (Beckers \& Homberg, 1991; Corthout, Uttl, Juan, Hallett, \& Cowey, 2000; Corthout, Uttl, Ziemann, et al., 1999; Laycock, Crewther, Fitzgerald, \& Crewther, 2007). This pre-stimulus TMS effect caused debate in the literature, because it was not easily explainable based on the assumed mechanism by which TMS induces its suppressive effect in the brain. Presumably, TMS exerts its effect by suppressing the stimulus-related signal in EVC (Harris, Clifford, \& Miniussi, 2008), or by adding random neural noise at the critical moment when a certain brain region attempts to process a given stimulus (Ruzzoli, Marzi, \& Miniussi, 2010). But if visual information has not reached EVC yet, there is no neural signal for TMS to interfere with. Therefore, even those few studies that 
incorporated such pre-stimulus time windows in their experimental design doubt that the effect they find is actually neural in nature. In fact, our own group set aside as eye blink artifacts the pre-stimulus TMS effect found in a study of visual motion discrimination (Sack, Kohler, Linden, Goebel, \& Muckli, 2006).

Recently, however, the idea has been put forward that awareness depends on the neural state that the cortex is in when the visual signal arrives (Mathewson, Gratton, Fabiani, Beck, \& Ro, 2009; Thut, Nietzel, Brandt, \& Pascual-Leone, 2006). The initiation of certain brain states is a conceivable mechanism of action of TMS. Generating a brain state that interacts with subsequent visual input might be a way in which TMS can influence awareness, even if the visual stimulus is not presented yet. These recent additions to the possible neural mechanisms of TMS led us to develop a renewed interest in the pre-stimulus TMS effect, and we wondered whether any empirical evidence exists that convincingly links it to eye blinks, as previously suggested, or to other non-neural effects TMS might have, such as multisensory integration of the visual stimulus with the clicking sound produced by TMS or an alteration in arousal or attention caused by the mildly aversive somatosensory sensations on the skull. It is reasonable to suggest that the auditory and somatosensory experiences that go along with TMS exert their effect in a time-dependent manner. They seem specifically relevant in the pre-stimulus period because they herald the appearance of the visual stimulus.

Although paramount for a better and appropriate understanding and interpretation of TMS-induced masking effects and the respective conclusions drawn for the neural mechanism underlying visual perception and awareness, only two studies have hitherto employed a measure of eye blink behavior during perceptual task execution. They demonstrated an early eye blink with an onset of approximately $10 \mathrm{~ms}$ post-TMS (Corthout, et al., 2000) and a late eye blink delayed by around 35ms (Beckers \& Homberg, 1991; Corthout, et al., 2000). Thus, a TMS pulse preceding the visual stimulus with roughly $10 \mathrm{~ms}$ or $35 \mathrm{~ms}$ can disturb perception by producing an eye blink at the moment of stimulus presentation. Unfortunately, the timing of the pre-stimulus masking effect is not consistent over studies, though in most cases it emerges in a rather narrow time window, particularly within single participants. This makes it hard to fit the eye blink data of Corthout et al. (2000) to the empirical results on perceptual tasks. Moreover, taking into account that an eye blink can take a full $200 \mathrm{~ms}$ (VanderWerf, Brassinga, Reits, Aramideh, \& Ongerboer de Visser, 2003) from initiation to end, any pulse as early as $200 \mathrm{~ms}$ pre-stimulus could hinder normal visual processing. Still, as yet, no studies have implemented eye tracking to identify specific blinks on a trial-by-trial basis to evaluate the 'clean' trials in isolation, even though this would allow the disentanglement of the eye blinks from neural TMS consequences. If the trials, in which TMS did not elicit an eye blink, still show a relative decrease in visual performance, the effect can no longer be attributed to eye blinks as such.

Performance deterioration due to occipital TMS has been taken as a marker for the breakdown of visual awareness. However, behavior does not necessarily reflect subjective experience and measures of the two might show divergent 
results. Therefore, studies of visual awareness do not limit themselves any longer to the investigation of merely behavioral measures. Lately, ratings of subjective awareness have been included as dependent variable (Boyer, Harrison, \& Ro, 2005; Christensen, et al., 2008; Koivisto, Mantyla, \& Silvanto, 2010) leading to intriguing outcomes. Boyer et al. (2005) describe a dissociation between visual discrimination performance and awareness, which they named TMS-induced 'blindsight'. It matches the symptoms of this neurological phenomenon in the sense that, even though people report to have no conscious percept, they are nevertheless able to identify the visual stimulus with accuracy levels surpassing chance. As mentioned before, a possible modus operandi of TMS might be the generation of particular cortical states. If the pre-stimulus TMS effect turns out to have a neural origin, it is conceivable that the induced state of the occipital cortex determines whether a stimulus reaches awareness or not. This process might be independent from the stimulus' behavioral consequences. Moreover, knowing that TMS can induce virtual blindsight, the effect TMS has on visual awareness might not show in the accuracy data. In this case, one needs a subjective measure of awareness to visualize the effect.

In the current study, we measured the effect of occipital TMS on a visual discrimination task over a wide range of time windows. We expected to find a breakdown in performance if magnetic stimulation followed visual presentation with an approximate delay of $80-100 \mathrm{~ms}$. Moreover, we tried to replicate the much less established pre-stimulus TMS effect in the same participant sample. We gathered electrophysiological data on participants' eye blinking behavior using electrooculographical recordings (EoG), to allow the post-hoc selection of trials without eye blinks. This way, we could systematically investigate whether TMS over EVC impairs visual performance both when applied at the critical time point after and before presentation of the visual stimulus, and whether these effects still hold after removal of eye blink trials. However, merely excluding eye blinks as causal to the pre-stimulus TMS effect is insufficient to ascribe it a neural nature. To be able to claim this, we included two control experiments. First, to control for the effects of auditory stimulation we included a sham TMS session. Second, in a new group of subjects the full experiment was repeated, but this time, we stimulated a site irrelevant to the visual discrimination task, namely vertex. This second control experiment allowed us to rule out other non-neural side effects of TMS, like somatosensation. To allow the comparison of objective versus subjective measures of visual awareness, introspective visibility ratings were acquired in addition to the objective accuracy data in the experimental sessions, as well as in the sham control and vertex control experiments. 


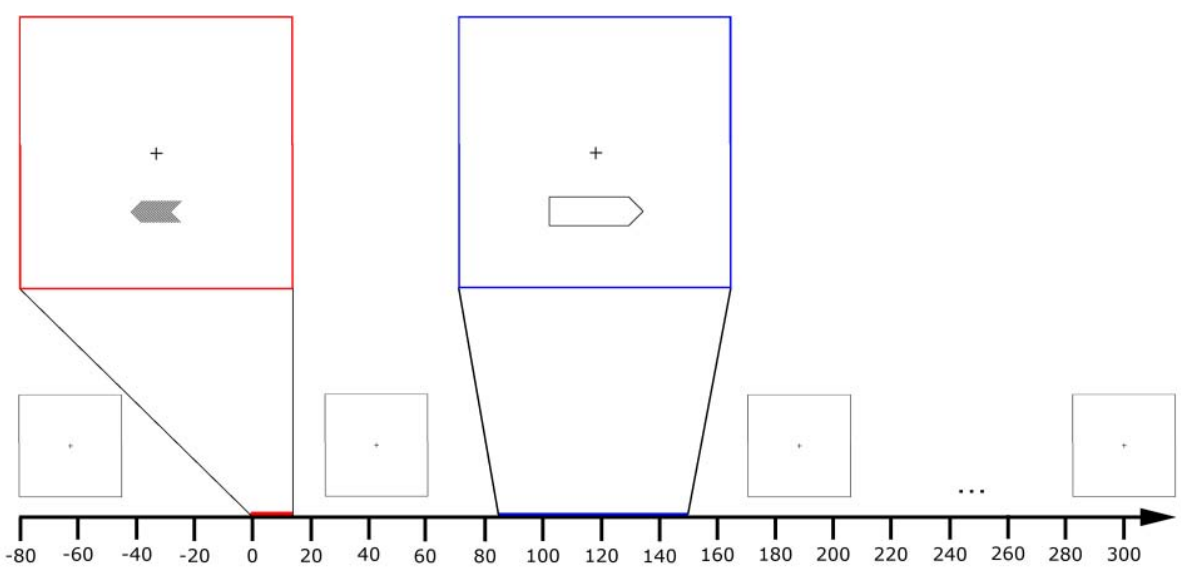

Time (ms)

Figure 1. Trial time line. A horizontal black arrow (S1) was presented below fixation for $16.7 \mathrm{~ms}$, followed by a larger horizontal arrow (S2) for $66.7 \mathrm{~ms}$ with an SOA of $83.3 \mathrm{~ms}$. During each trial a single TMS pulse was delivered over EVC at any of 20 different time points time-locked to S1 (range from -80 to $200 \mathrm{~ms}$ in steps of $20 \mathrm{~ms}$ ). Participants were to indicate via button press whether they consciously perceived S1 and in which direction, left- or rightward, they believed it to point.

\section{MATERIALS AND METHODS}

\section{Experiment 1: Early visual cortex stimulation}

\section{Participants}

Ten healthy participants (two males, mean age 22.1y, range 19-26) including one of the authors (CJ) with normal or corrected-to-normal vision participated in this study. All participants were screened by the medical supervisor prior to participation. They gave their written informed consent and were compensated financially. The study was approved of by the Medical Ethics Committee of the University Medical Center, Maastricht, the Netherlands.

Two participants were excluded from further analysis, when their baseline accuracy proved to be below 75\% correct responses. Such a low baseline score might cause floor effects, which could mask any effects due to magnetic stimulation. 


\section{Stimuli}

On each trial, two consecutive stimuli (S1 and S2) were presented in the horizontal center of the visual field, slightly $\left(0.8^{\circ}\right.$ visual angle) below fixation. $\mathrm{S} 1\left(120 \mathrm{~cd} / \mathrm{m}^{2}\right.$; contrast $\left.68 \%\right)$ was a small $\left(0.8^{\circ}\right.$ by $\left.1.86^{\circ}\right)$ arrow stimulus, S2 a larger $\left(1.09^{\circ}\right.$ by $\left.3.47^{\circ}\right)$ stimulus made up of outer contours only (see Figure 1$)$. Both stimuli were oriented horizontally and pointed either in leftward or rightward direction. S1 was presented for $16.7 \mathrm{~ms}$, S2 for $66.7 \mathrm{~ms}$ with a stimulusonset asynchrony of $83.3 \mathrm{~ms}$. S1 and S2 were congruent, i.e. had an identical orientation, on half of the trials. The presentation of S2 was included, because at a later stage, the results of this task will be compared to another task, data of which were collected in a separate session (see Chapter 3). In order to keep both set-ups as identical as possible we decided to include S2, even though it is not relevant to the task under discussion here.

Stimuli appeared on a 17" TFT, Samsung SyncMaster 931 DF with a refresh rate of $60 \mathrm{~Hz}$ at a viewing distance of $61 \mathrm{~cm}$. Calibration measurements using a photodiode on the monitor and concurrent measurement of the external TMS triggering signal leaving the parallel port of the stimulus-PC showed a constant, stable and reliable offset of $2 \mathrm{~ms}$ for all stimulus-TMS SOAs. When we discuss SOAs in the remainder of the chapter, we refer to the SOAs as requested of our presentation software. All stimuli were presented and behavioral responses recorded via the Presentation software package (Neurobehavioral Systems, Inc., Albany, CA).

\section{Task}

Participants performed a two alternative forced choice (2AFC) visual discrimination task. They were comfortably seated in front of the computer screen with their heads fixed in a chin-rest. Participants were instructed to fixate throughout the experiment and to respond on each trial as accurately as possible.

First, participants were to state via button press whether they saw S1 consciously or not. All participants were instructed to respond positively to this question when their percept entailed some informational content, even if they would still be unsure on stimulus direction. With their second response, participants indicated of which orientation, i.e. left or right, they believed S1 to be. Participants were explicitly informed that the two responses were independent, in the sense that by indicating that S1 was perceived, they were not necessarily expected to give a correct answer on its direction, or vice versa. The program did not proceed to the next trial unless both responses were given by the participant. A single TMS pulse was delivered to the participant's occipital cortex on each trial, the timing of which varied from -80 to $300 \mathrm{~ms}$ post S1onset in steps of $20 \mathrm{~ms}$ (see TMS protocol).

Data were acquired in two separate sessions to prevent fatigue, as a total of 840 trials were collected per participant. Before the start of the experiment, participants were asked to train with the task until they scored an average correct percentage of over $80 \%$ on 3 consecutive blocks of 20 trials with a 
maximum of 15 blocks. If participants failed to reach this accuracy threshold within 15 blocks, they were not included in the rest of the study. During training, single trial performance was fed back to the participants by means of the fixation cross turning green (i.e. correct response) or red (i.e. incorrect response). At the end of each block summary feedback was presented on screen.

Following training, participants executed a No TMS baseline measurement. Participants executed 20 trials without feedback and without magnetic stimulation. The next run consisted of 400 TMS trials, split into 20 blocks of 20 trials. For each TMS time window, including the No TMS baseline, data on 20 trials were collected in each of two sessions, accumulating to a total of 40 trials per time window. Trial order was randomized and inter-trial interval was jittered with an average duration of $5000 \mathrm{~ms}$.

\section{TMS Protocol}

Before the start of the experiment, the site of TMS stimulation was determined by means of phosphene localization. The TMS coil was placed against the back of the participant's head, approximately $1 \mathrm{~cm}$ above the inion. While fixating on the center of a computer screen, the participant was asked to indicate whether phosphenes were induced by single TMS pulses and if so, where these phosphenes appeared in the visual field. Coil position was varied until perceived phosphenes overlapped with the location in the visual field where the experimental stimuli were later presented. If full overlap could not be achieved, the coil position optimal for inducing phosphenes closest to the target location was chosen. If phosphene localization is used as a procedure to determine the coil position, V2 and V3d have been found to be the brain areas mostly affected by magnetic stimulation (Thielscher, Reichenbach, Ugurbil, \& Uludag, 2009).

During the actual experiment, a single TMS pulse was delivered on each trial at any of 20 different time points. The moment of stimulation was time-locked to $\mathrm{S} 1$ and ranged from -80 to $300 \mathrm{~ms}$ post-S1 onset in steps of $20 \mathrm{~ms}$. All pulses were delivered with a figure-of-eight coil (MC-B70, the inner and outer radii of the two coil loops are 1.2 and $5.4 \mathrm{~cm}$, respectively). Pulse intensity was set to a fixed value of $70 \%$ of the maximum output of the Medtronic MagPro X100 stimulator (Medtronic Functional Diagnostics A/S, Skovlunde, Denmark; maximum stimulator output $=1.9 \mathrm{~T}$ ). This intensity was chosen because it much exceeds the average phosphene threshold (PT) measured with this type of stimulator. Because we know that TMS induced masking occurs with much higher stimulation intensities than phosphene generation (Kammer, 2007; Kammer, Puls, Erb, \& Grodd, 2005), this intensity is likely to induce visual suppression without generating phosphene perception in our participants. The average PT of four of our participants is $51 \%$ maximum stimulator output. Afterwards, participants were asked whether they experienced any effects of the TMS pulse when performing the task. All participants reported that they did not perceive the stimulus accurately on part of the trials, which they attributed to the TMS pulse, but none of them reported seeing phosphenes. 


\section{Electrooculography}

Electrooculographical (EoG) data were recorded via two $\mathrm{Ag}$ - $\mathrm{AgCl}$ electrodes attached above and below the right eye and a BrainAmp ExG amplifier (BrainProducts GmbH, Munich, Germany) with a sampling rate of $1000 \mathrm{~Hz}$. A third electrode was placed on the mastoid behind the right ear and served as a reference electrode. Data were acquired with BrainVision Recorder software (BrainProducts GmBh, Munich, Germany). Data were filtered online with a high-pass filter (cut-off frequency $0.1 \mathrm{~Hz}$ ) and a low-pass filter of $10 \mathrm{~Hz}$. Offline data analyses were performed using BrainVision Analyzer v2.0 (BrainProducts GmBh, Munich, Germany) and Matlab R2007a (MathWorks, Natick, Massachusetts, U.S.A) software. Epochs of $300 \mathrm{~ms}$ (-200 to 100 post S1-onset) were created and baseline corrected relative to the preceding $200 \mathrm{~ms}$ (i.e. -400 to -200 $\mathrm{ms}$ post S1-onset). Threshold values were taken to be 0.33 times the median of the 10 highest and lowest values in the recording. If any of the 300 selected data points in an epoch exceeded either of the two thresholds, that trial was classified as a 'blink' trial. This manner of EoG data analysis has proven to correctly identify eye blinks on $94.3 \%$ of the cases (Skotte, Nojgaard, Jorgensen, Christensen, \& Sjogaard, 2007). As a conservative approach, we decided to look $200 \mathrm{~ms}$ back from S1 onset, because eye blinks have been shown to have a depressant effect on visual cortex for about $150 \mathrm{~ms}$, so any prior eye blinks may still exert an effect on current $\mathrm{S} 1$ processing.

\section{Experiment 2: Sham TMS Control}

TMS can lead to multisensory enhancement due to the clicking sound. To control for this potential confounder, an additional control in the form of sham TMS was included. Six out of ten (one male, mean age 22.8y, range 20-27) original participants participated once more. Participants' performance level was assessed by having them go through a similar training protocol as in the experimental sessions. After training, they completed a No TMS baseline run of 40 trials. In the final TMS run, the sham TMS coil (MC-P-B70 Placebo) was placed over the backside of the participants' head, and during each trial the participants received one sham TMS pulse. To optimally match the sound properties of real and sham TMS, the TMS intensity was set to a level at which the volume in decibels of the sham TMS pulse corresponded with the intensity used in the original experimental sessions. The sham TMS pulse was randomly delivered at any of the original pre-stimulus time windows $(-80,-60,-40,-20,0 \mathrm{~ms}) .40$ trials per time window were collected.

\section{Experiment 3: Vertex TMS control}

A second control experiment was executed to control for the somatosensory sensations participants experience on their scalp as a result of skin and tiny muscle stimulation by the TMS pulse. A viable control for this type of TMS by- 
product is magnetic stimulation at a non-relevant location. During sham TMS the scalp of the participants is not subjected to a magnetic field of any kind and therefore, sham TMS does not elicit the same sensations as real TMS.

A group of eight new participants (four males, mean age 25.5y, range 22-32) was recruited. Stimuli, task and TMS protocol were identical to Experiment 1, with the exception of TMS stimulation site. Vertex was determined as the electrode position $\mathrm{Cz}$ in the 10-20 EEG positioning system. In a small number of participants, this induced muscle twitches in their leg, probably due to stimulation of the leg area of the primary motor cortex, which is located on the dorsal part of the pre-central gyrus. In these cases the TMS coil was moved in posterior direction until the twitching in leg muscle ceased.

One participant was excluded from further analysis, because her baseline accuracy proved to be below $75 \%$ correct responses. Such a low baseline score might cause floor effects, which could mask any effects due to magnetic stimulation.

\section{RESULTS}

\section{EoG}

A time interval ranging from $200 \mathrm{~ms}$ prior until 100 post S1-onset was screened for eye blinks, as any blink within this interval might hinder visual perception of the target stimulus. In Experiment 1, participants blinked on $8 \%$ of the trials in this crucial $300 \mathrm{~ms}$ epoch on average, which might have prevented them from consciously perceiving the target stimulus. Across participants, the frequency of eye blinks tended to vary considerably, ranging from 1.1 to $33.3 \%$ of the total number of trials. As expected, eye blink frequency turned out to be higher for trials in which TMS was applied in the early time windows (i.e. -80 to $20 \mathrm{~ms}$, mean $=21.9 \%$, see Figure 2.B) than in the later ones. A Greenhouse-Geisser corrected repeated-measured one-way ANOVA of the factor TMS time window on eye blink frequency did not prove significant, although it revealed a trend $(\mathrm{F}(1.778, .492)=3.615, \mathrm{p}=.094)$.

In Figure 2.A an average EoG waveform is plotted for 'blink' and 'no blink' trials in a single participant and for a single TMS time window $(-60 \mathrm{~ms})$. Even in the 'no blink' trials some muscle twitching takes place, though this cannot be considered a full-fledged eye blink. Moreover, in this participant, it takes over $150 \mathrm{~ms}$ for the signal to get back to baseline following a TMS pulse. Hence, one would predict that if TMS-induced eye blinks cause visual suppression, they do so when TMS is applied over a wide temporal range. 

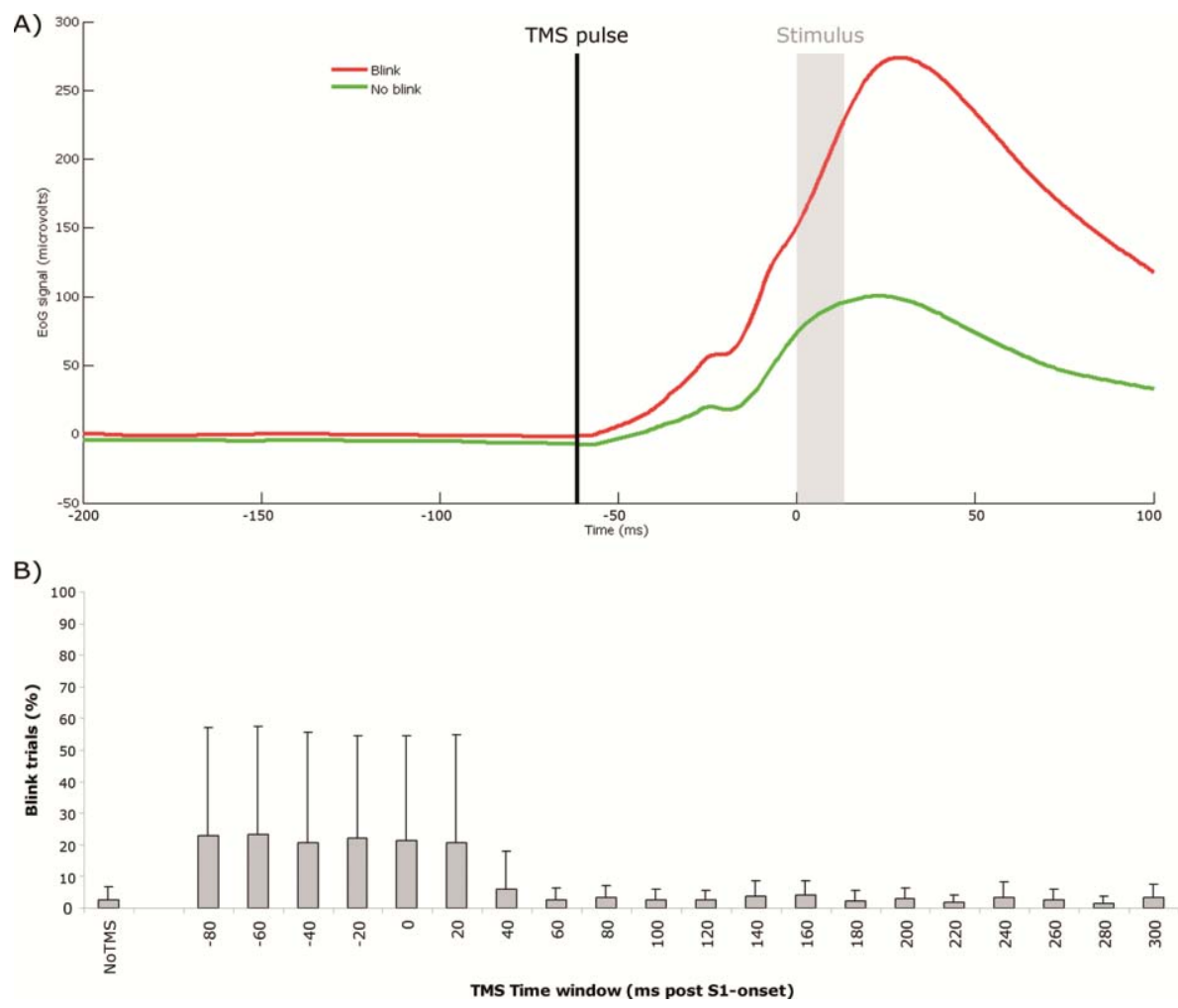

Figure 2. EoG example wave forms and eye blink frequency. A) Average EoG waveforms for the TMS $-60 \mathrm{~ms}$ condition in a single participant. The black line indicates the moment of TMS pulse delivery. The shade covers the period of stimulus presence. The participant blinked (red line) on 13 of 40 trials, with a peak amplitude of $273.8 \mu \mathrm{V}$. Peak amplitude for 'no blink' trials (green line) was $100.4 \mu \mathrm{V}$ (difference $=173.4 \mu \mathrm{V})$. B) Average percentage of 'blink' trials per TMS time window over all participants. Error bars indicate standard deviations. A trial was considered a 'blink' trial if the EoG signal crossed a certain threshold (see Methods) in a time frame of -200 to $100 \mathrm{~ms}$ post S1onset. Eye blink frequency was highest for pre-stimulus time windows. This was to be expected, since TMS is known to induce eye blinks.

\section{Behavioral data}

\section{Accuracy}

A one-way repeated-measures ANOVA was performed over the average percentage correct responses per TMS time window for the remaining 8 subjects of Experiment 1 . Since equal variances could not be assumed, Greenhouse-Geisser correction was applied. The factor TMS time window showed a significant effect $(\mathrm{F}(3.468,24.273)=4.446, \mathrm{p}=.01)$. Post hoc analysis, comparing all TMS time windows to baseline (No TMS) revealed a decrease in performance for the classical $80 \mathrm{~ms}(\mathrm{p}=.039), 100 \mathrm{~ms}(\mathrm{p}=.011)$ and $120 \mathrm{~ms}(\mathrm{p}=.031)$ post-stimulus 
time windows. Furthermore, performance was significantly decreased for 3 out of 4 pre-stimulus time windows, i.e. $-80 \mathrm{~ms}(\mathrm{p}=.010), 60 \mathrm{~ms}(\mathrm{p}=.012)$, and $40 \mathrm{~ms}(\mathrm{p}=.021)$. Note that alpha values of all post-hoc comparisons reported here are uncorrected for multiple comparisons.

Next, data were filtered based on whether subjects blinked in the selected $300 \mathrm{~ms}$ around S1. A paired-samples t-test on the average number of correctly performed trials pooled over time windows showed that participants on average scored 9.7\% lower on 'blink' trials (mean $=76.1 \%$ ) versus 'no blink' trials (mean $=85.8 \%, t(7)=-2.644, p=.033$ ) (see Figure 3.A).

After removal of all eye blink trials from the Experiment 1 dataset, the main effect of TMS time window on accuracy was still significant $(F(3.376,20.256)=$ 4.083, $\mathrm{p}=.017)$. The mean accuracy for post-stimulus time windows $80 \mathrm{~ms}(\mathrm{p}=$ $.009)$ and $100 \mathrm{~ms}(\mathrm{p}=.010)$ differed significantly from baseline. TMS time window $120 \mathrm{~ms}$ did not prove significant any longer, probably due to a slight loss of power resulting from the exclusion of eye blink trials. However, TMS administered $120 \mathrm{~ms}$ after stimulus presentation still showed a strong trend towards decreased performance $(\mathrm{p}=.053)$. Moreover, applying TMS $60 \mathrm{~ms}(\mathrm{p}=$ $.023)$ or $80 \mathrm{~ms}(\mathrm{p}=.042)$ before stimulus-onset led to a significant decrease in performance. The critical pre-stimulus TMS time window tended to shift somewhat over participants (see Figure 4), which caused the group data to show a rather broad time window of TMS suppression.

To investigate whether any sub threshold EoG activity in the 'no blink' trials influenced task performance, we split the pre-stimulus TMS trials into 4 bins based on EoG peak amplitude, and then plotted performance as a factor of bin. For the critical TMS time windows $-80,-60$ and $-40 \mathrm{~ms}$, visual discrimination performance did not linearly depend on EoG amplitude, but rather showed an asymptotic trend. The bin of highest EoG amplitude included all trials classified as 'blink' trials. Performance in these trials was significantly worse $(p=.019)$ than for those in the second bin. Performance then reached a plateau and no further performance increase occurred from bin 2 to bin 3 or 4 . This analysis rules out that sub threshold EoG activity accounts for some of our pre-stimulus TMS effects on visual perception.

A Greenhouse-Geisser corrected one-way repeated-measures ANOVA on the data of Experiment 2 did not reveal a significant effect of the factor sham TMS time window (6 levels) on accuracy scores $(\mathrm{F}(1.195,5.977)=2.080 ; \mathrm{p}=.203)$, indicating that TMS-related auditory stimulation does not explain the prestimulus TMS effect found in Experiment 1.

Analysis of the vertex TMS data (Experiment 3) excluded further nonneural TMS effects. Again, Greenhouse-Geisser corrected repeated-measures ANOVA did not reveal any significant effect of TMS time window (F(4.119, $24.715)=1.906, p=.140$ ) (see Figure 5.B). The disappearance of the poststimulus TMS effect from the EVC to vertex stimulation data validates vertex as a task-irrelevant control site that does not interfere with processing of visual information. 
A)

Accuracy
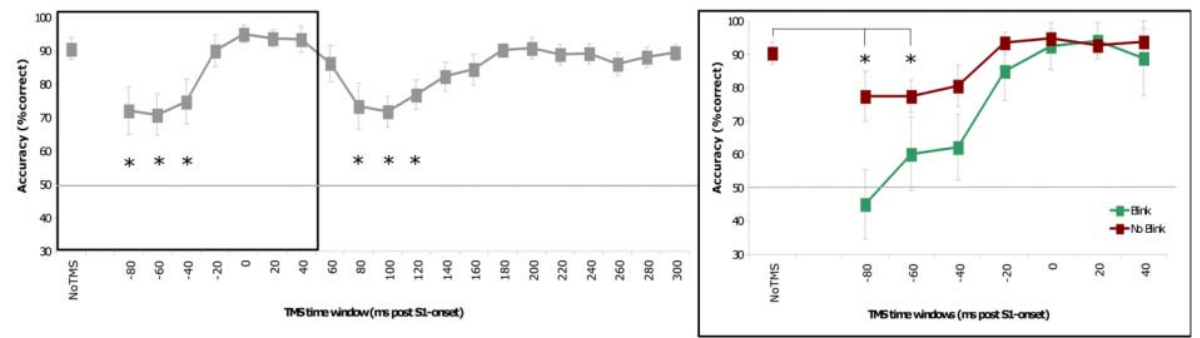

B)

Awareness

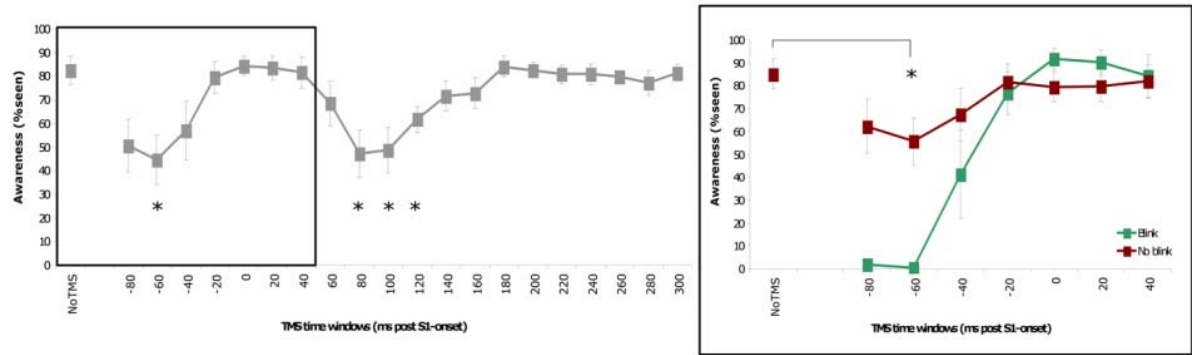

Figure 3. Behavioral results for 'blink' and 'no blink' trials A) Average accuracy scores per time window expressed in percentage of correct responses. The left panel shows data of all trials. Trials were sorted post hoc based on the presence or absence of eye blinks. Sorted data are presented in the right panel: the green line represents the data of all trials which contain eye blinks; the red line represents the data of all trials which did not contain eye blinks. B) Average awareness per time window expressed in percentage seen stimuli. The left panel shows data of all trials. Trials were sorted post hoc based on the presence or absence of eye blinks. Sorted data are presented in the right panel: the green line represents the data of all trials which contain eye blinks; the red line represents the data of all trials which did not contain eye blinks.

\section{Awareness}

For Experiment 1, a one-way repeated-measures ANOVA was performed over the average subjective awareness responses per TMS time window, again Greenhouse-Geisser corrected. As on accuracy, TMS time window had an effect on subjective awareness rating $(\mathrm{F}(2.603,18.219)=4.750, \mathrm{p}=.016)$. Awareness was significantly impaired when TMS was applied $80 \mathrm{~ms}(\mathrm{p}=.016), 100 \mathrm{~ms}(\mathrm{p}=$ $.014)$, and $120 \mathrm{~ms}(\mathrm{p}=.034)$ post-stimulus. In addition, subjective awareness was significantly suppressed when TMS was applied 60ms ( $\mathrm{p}=.016)$ before presentation of the visual stimulus.

Participants were unaware of the visual stimulus on a relatively larger part of the 'blink' trials (mean $=51.7 \%$ ) than on the 'no blink' trials (mean $=73.4 \%$ ), as a paired-samples t-test showed $(t(7)=-5.131, p=.001$ ) (see Figure 3.B). 


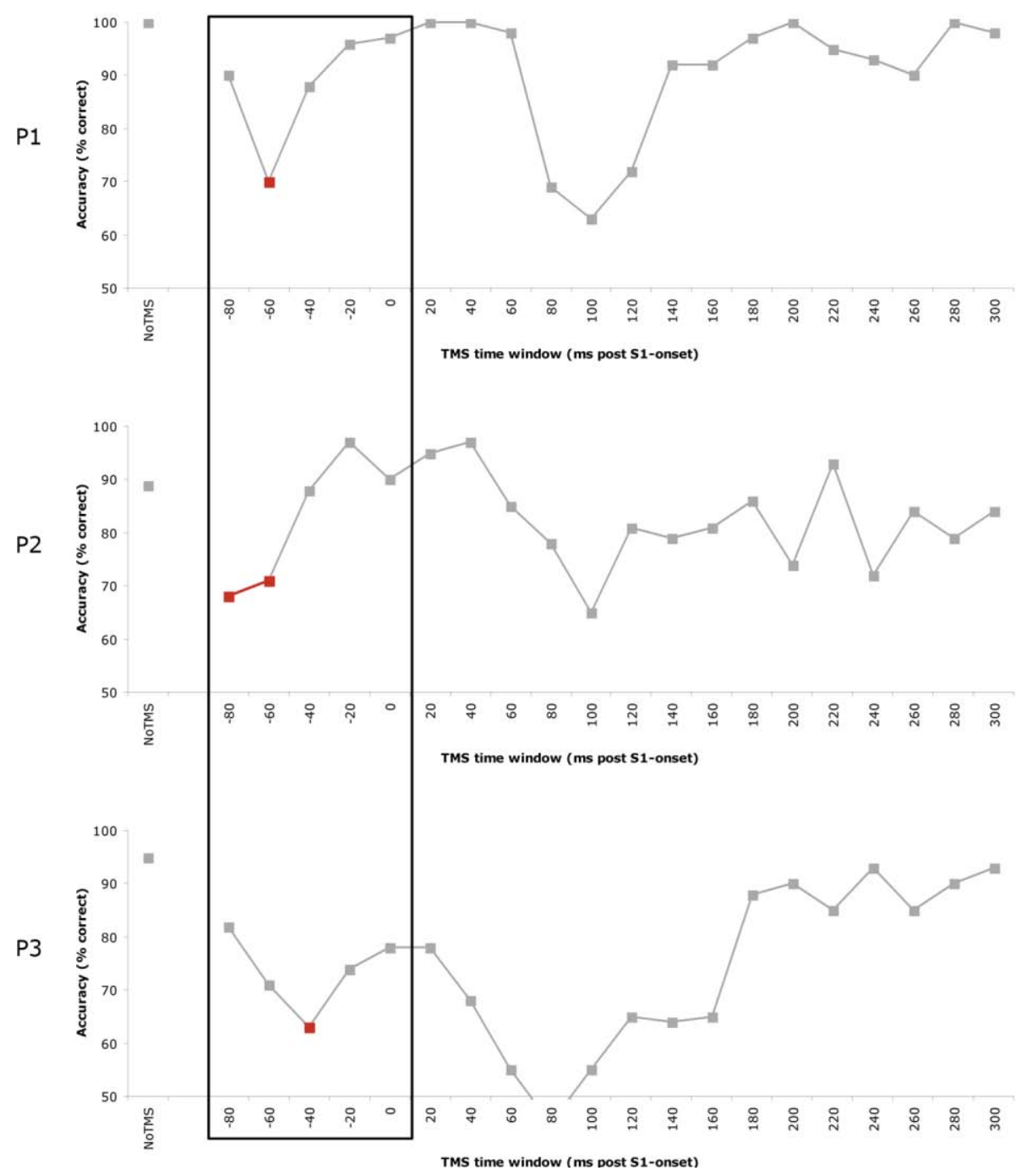

Figure 4. Individual data of three participants. These data show the temporal specificity of the prestimulus TMS effect. P1 shows the greatest performance dip at $-60 \mathrm{~ms}$, P2 at -80 and $-60 \mathrm{~ms}$, and P3 at $-40 \mathrm{~ms}$. This shift in pre-stimulus time window over participants can explain why we find a rather broad effective TMS time window (i.e. -80 to $-40 \mathrm{~ms}$ ) when only considering the group data.

After removal of all eye blink trials from the Experiment 1 dataset, the ANOVA main effect of TMS time window on subjective awareness ratings was still significant $(\mathrm{F}(2.334,16.273)=4.525, \mathrm{p}=.02)$ with a significant decrease in the average number of consciously perceived stimuli for the $80 \mathrm{~ms}(\mathrm{p}=.006), 100$ $\mathrm{ms}(\mathrm{p}=.005)$, and $120 \mathrm{~ms}(\mathrm{p}=.023)$ post-stimulus time windows, respectively. 


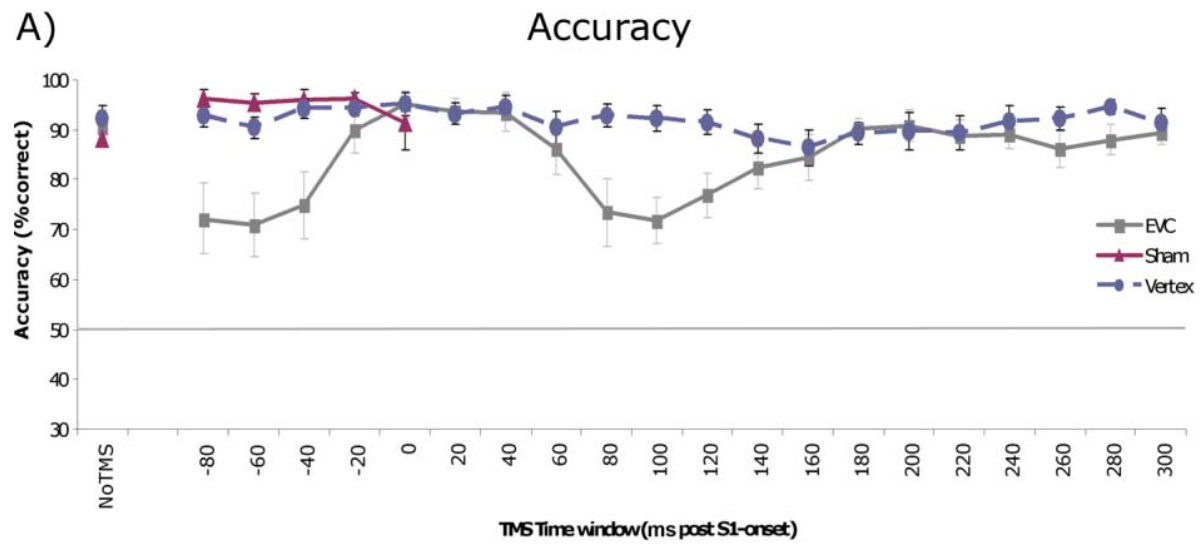

B)

Awareness

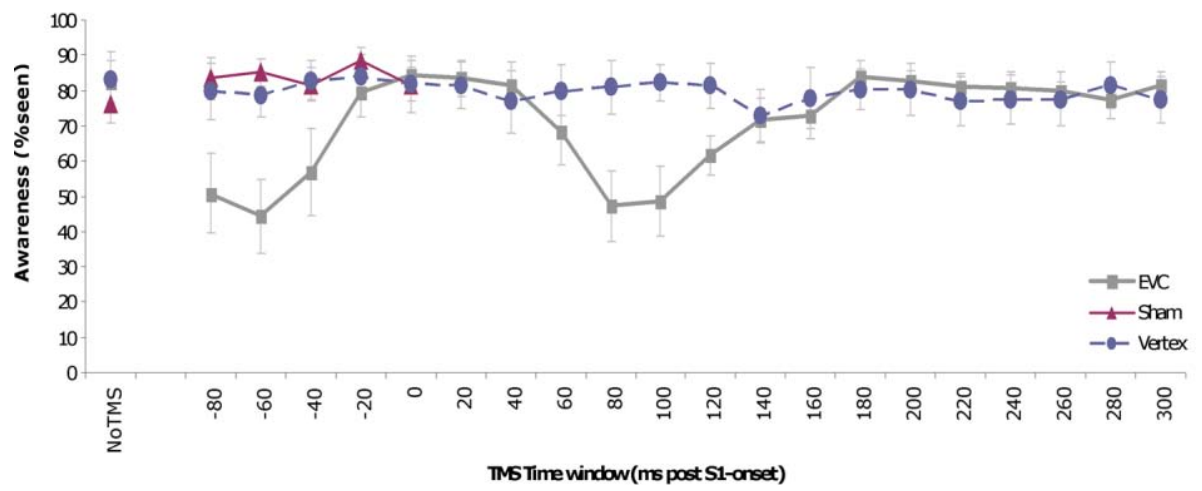

Figure 5. Control data. A) Average accuracy scores per time window expressed in percentage of correct responses. Lines represent the experimental data (EVC stimulation; grey line) and the data of both control experiments (sham TMS and vertex TMS; purple and dark blue lines, respectively). Contrary to the results of EVC stimulation, TMS pulse timing did not affect accuracy in either of the two control experiments. B) Average awareness per time window expressed in percentage seen stimuli. Lines represent the experimental data (EVC stimulation; grey line) and the data of both control experiments (sham TMS and vertex TMS; purple and dark blue lines, respectively). Contrary to the results of EVC stimulation, TMS pulse timing did not affect subjective awareness in either of the two control experiments.

The same effect was also still significant for the pre-stimulus time window at $60 \mathrm{~ms}$ before visual stimulus onset $(\mathrm{p}=.043)$.

An additional analysis was performed on the 'no blink' data of Experiment 1. For all the time windows in which participants reported to be unaware of the stimulus on approximately 50 percent of the trials $(-80,-60,-40,80,100$, $120 \mathrm{~ms}$ ), one-tailed one sample t-test with chance level, i.e. 50, as test value was performed on the average accuracy data of the unaware trials. The average accuracy proved significantly higher than $50 \%$ for the $-40 \mathrm{~ms}(\mathrm{t}(6)=2.159, \mathrm{p}=$ .037 ) pre-stimulus time window, as well as for the $80(\mathrm{t}(6)=2.071, \mathrm{p}=.042)$ 


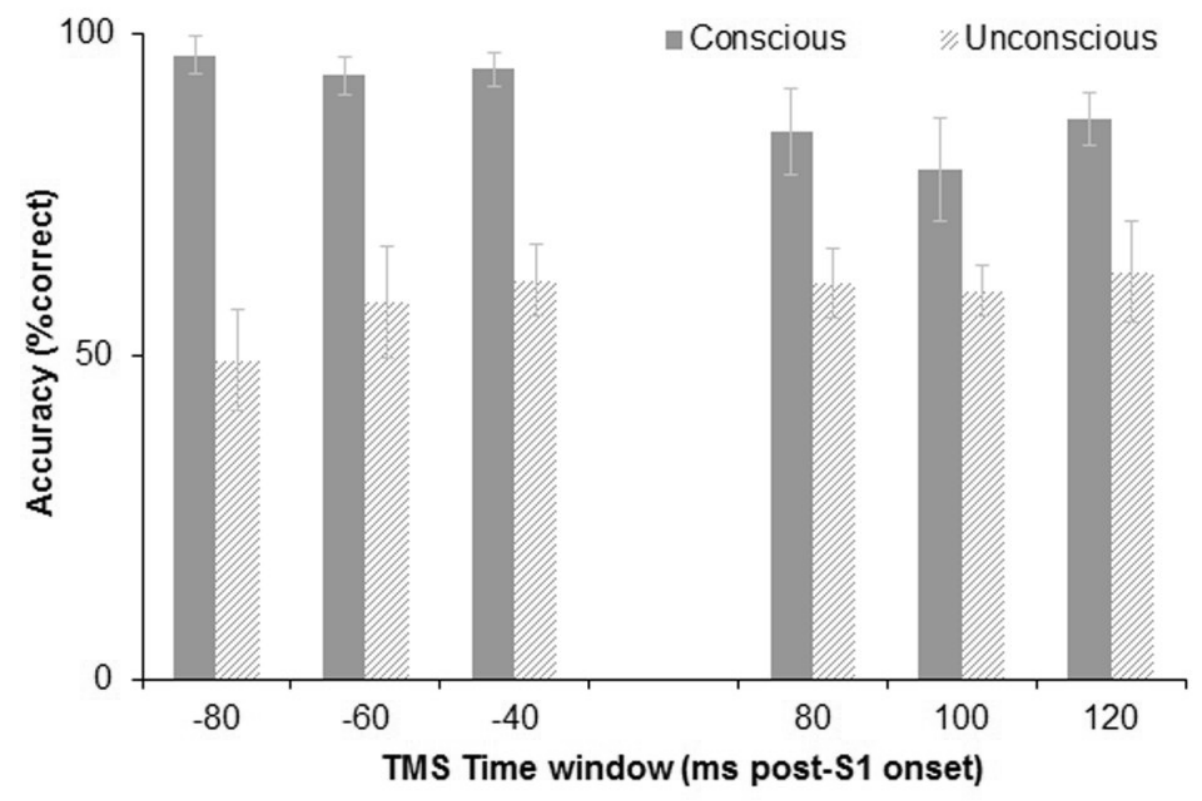

Figure 6. Performance scores on aware and unaware trials. Data were split based on participants' awareness report for three pre- and three post-stimulus time windows in which participants reported awareness on approximately half of the cases. Solid bars reflect average accuracy scores when participants reported to be aware of the target stimulus, striped bars reflect average accuracy scores when participants reported to be unaware of the target stimulus. Participants were more accurate on aware versus unaware trials, even though performance remained above chance level for the $-40,80$ and $100 \mathrm{~ms}$ TMS time windows.

and $100 \mathrm{~ms}(\mathrm{t}(6)=2.676, \mathrm{p}=.019)$ post-stimulus time windows, whereas the $120 \mathrm{~ms}$ time window showed a similar trend $(\mathrm{t}(6)=1.656, \mathrm{p}=.075)$ (see Figure $6)$.

A one-way repeated-measures ANOVA on the data of Experiment 2 did not reveal a significant effect of the factor sham TMS time window (6 levels) on subjective awareness $(F(5,25)=2.009 ; p=.112)$. Moreover, Greenhouse-Geisser corrected repeated-measures ANOVA on the data of Experiment 3 did not reveal any significant effect of vertex TMS $(F(2.801,16.804)=1.249, p=.322)$ (see Figure 5.B).

\section{DISCUSSION}

The current study reliably reports a suppressive TMS effect in a visual discrimination task at the classical (i.e. $80-100 \mathrm{~ms}$ ) time window post-stimulus onset as well as at the much less established pre-stimulus time window (-60 ms). These results add evidence to the reality of such a pre-stimulus effect and 
validate the necessity of carefully looking into pre-stimulus time windows when investigating visual awareness with TMS. A very small number of studies so far have indeed looked into pre-stimulus effects (Beckers \& Homberg, 1991; Corthout, et al., 2000; Corthout, Uttl, Walsh, et al., 1999; Corthout, Uttl, Ziemann, et al., 1999; Laycock, et al., 2007; Sack, et al., 2006; Stevens, McGraw, Ledgeway, \& Schluppeck, 2009), although most of them have disregarded the effect as eye blink related. In fact, in a recent study published by our own group (Sack et al., 2006), TMS was found to impair visual discrimination performance when applied over V5/hMT+ $40 \mathrm{~ms}$ prior to motion onset; a result we considered to be most likely due to eye blink artifacts. In the current study, we tested this interpretation directly by incorporating an electrophysiological measure of eye blinking during TMS-induced visual awareness suppression. Even if we are not the first to do so (Beckers \& Homberg, 1991; Corthout, et al., 2000), none of these earlier studies sorted the trials based on the eye blink data and analyzed the eye blink-free trials in isolation. By doing exactly this, we were able to conclude that the pre-stimulus TMS effect is still present after all trials with eye blinks were removed from the data. Therefore, eye blinks simply cannot account for the pre-stimulus effect reported here.

Eye blinks are not the only by-product of magnetic brain stimulation. Changes in arousal levels or attentional focus as well as multisensory interaction can occur due to the somatosensory skull sensation and clicking sound accompanying the TMS pulse. To exclude the possibility that any of these phenomena caused the pre-stimulus suppression we uncovered for both measures of visual awareness, we conducted two additional control experiments; a sham stimulation experiment that controlled for any non-neural effects related to the clicking of the TMS coil and a vertex stimulation experiment that controlled for any non-neural effects related to the TMS-induced somatosensory experiences. Both experiments failed to show an effect of TMS pulse timing. Thus, we can safely conclude that neither eye blinks, multisensory integration, nor somatosensory stimulation can account for the pre-stimulus effect of EVC TMS.

Which alternative, neural, mechanism underlies this early TMS effect? Corthout et al. (2000) pose a number of possible mechanisms of which they deem a post hoc neural suppression, which eye blinks are known to have on visual cortex (Volkmann, Riggs, \& Moore, 1980), most plausible. This would mean, however, that they still assume the occurrence of an eye blink on most, if not all, trials. The current results do not support this assumption. For the pre-stimulus time windows, we find an eye blink on approximately 25 percent of the trials.

Laycock et al. (2007) found that TMS over V1/V2 applied 42ms prior to stimulus onset negatively affects performance on a motion detection task. They explained their results as an inhibition of expectation. A frontoparietal attentional network might already bias lower level visual cortex in favor of an anticipated visual stimulus. Supposedly, it is this neuronal bias that pre-stimulus TMS interferes with. One would expect magnetic stimulation to show an effect in a rather broad range of time prior to target onset. However, as depicted in Figure 4, this effect is rather time-specific and therefore, difficult to reconcile with the concept of attentional disruption. 
Another neural account was put forward by Stevens et al. (2009). They stimulated $\mathrm{V} 5 / \mathrm{hMT}+$ while participants were performing a task of global motion processing and found performance decrease when TMS was applied $60 \mathrm{~ms}$ before the stimulus appeared on screen. The authors attribute this to the feedback connections running from V5 towards V1 and propagating the TMS effect induced in V5/hMT+. According to this line of reasoning, TMS exerts its disruptive influence on signal processing not in the stimulated area, since the signal has not arrived there yet. Rather, the TMS effect travels backward in the processing hierarchy where it meets the neural signal in lower level cortex and disturbs its processing in this remote brain area. Although V1 was not the stimulated brain area in this particular study, the idea of backprojections transporting the neural effects of TMS to lower level areas, is applicable to V1, and thus our current study, as well. Massive bundles of feedback connections run from V1 back to the thalamus, where they terminate in lateral geniculate nucleus (LGN). Alternatively, instead of via these backpropagations, the TMS effect might travel anthrodromically through the axons connecting LGN to striate cortex in a feedforward fashion (Cowey, 2008).

A number of neuroscientific studies have led to the emerging idea that processing of neural input interacts with the state the cortex is in (Busch, Dubois, \& VanRullen, 2009; Mathewson, et al., 2009; van Dijk, Schoffelen, Oostenveld, \& Jensen, 2008). The state-dependency concept has even led to the development of new applications of TMS in cognition research, which exploit the fact that the state of the cortical target area influences the neurophysiological effect of magnetic stimulation (Cattaneo \& Silvanto, 2008a, 2008b; Silvanto \& Muggleton, 2008; Silvanto, Muggleton, Cowey, \& Walsh, 2007; Silvanto \& Pascual-Leone, 2008). If we view the current experiment in this state-dependent light, an additional account of the results comes into sight. Supposing the prestimulus TMS pulse induces a certain state in EVC, which hinders the processing of the afterward presented visual stimulus, we wondered about the nature of this TMS-evoked neural state. Based on a number of studies combining TMS with electrophysiological measures, we speculate that the pre-stimulus TMS may exert some local oscillation rhythm which frequency and phase may directly interfere with post-TMS processing of incoming visual input, with respective consequences on behavior. Because visual awareness depends on the state occipital cortex is in, when it receives the sensory information, it is plausible that pre-stimulus TMS affects visual awareness by inducing such a local oscillation rhythm that hinders perception of later presented visual stimuli. More concretely, EEG studies have detected an inverse correlation between power in the alpha frequency band $(8-14 \mathrm{~Hz})$ and visual awareness (Thut, et al., 2006). Thus, the more reduced the alpha power in the contralateral hemisphere, the more accurate the detection of a visual stimulus. Alpha in the ipsilateral hemisphere correlates positively with performance. Moreover, it has recently been shown that occipital TMS in fact evokes posterior alpha activity (Rosanova, et al., 2009) and that any occipital cortical excitability changes due to TMS interact with alpha power at the moment of pulse delivery (Romei, et al., 2008). Mathewson et al. (2009) have even found that, if occipital alpha power is high, the wave phase is predictive of subsequent visual awareness, which could 
nicely account for the temporal discreteness of the pre-stimulus TMS effect we report here. Hence, TMS might specifically exert an effect, if the visual stimulus is presented in the inhibitory phase of the consequential alpha wave. Our current results nicely fit this line of reasoning, because they show the prestimulus TMS effect to be both time-specific and of neural origin.

Awareness ratings as well as discrimination performance seemed to behave strikingly similar over the whole range of tested TMS time windows. However, if we consider the accuracy scores per level of awareness per time window, it turns out that even if TMS reduced awareness, this does not mean that accuracy dropped to chance level altogether. These data are nicely in line with the original study by Boyer et al. (2005) uncovering 'TMS-induced blindsight'. Moreover, the phenomenon proved to behave in a time-specific manner, especially in the pre-stimulus period, when it only appeared if the TMS pulse preceded the visual stimulus by $40 \mathrm{~ms}$. However, instead of a genuine dissociation between self-reported awareness and performance, it could rather reflect a difference in sensitivity between both subjective and objective awareness measures, especially considering that participants had to make a binary judgment on their subjective awareness.

Koivisto, Railo, \& Salminen-Vaparanta (2011) had participants discriminate symbolic arrow stimuli comparable to the ones of the current study, but their results do not support pre-stimulus TMS masking. However, only a single prestimulus TMS time window was tested. Moreover, the data displayed a prestimulus performance dip that failed to reach significance, raising statistical power concerns. Our post-stimulus TMS masking effect is nicely in line with the performance decrease Koivisto et al. (2011) found, when they applied TMS $90 \mathrm{~ms}$ post-stimulus onset. Because the authors also included a subjective awareness measure in their design, they were able to investigate the average discrimination accuracy for the unseen arrows in this particular time window. Contrary to our findings and those of Boyer et al. (2005), they did not find above chance level performance, possibly due to their measure of subjective awareness being more sensitive, as participants were asked to indicate visual awareness on a 4-point scale.

The current body of research on the interaction of self-reported visual awareness and performance focuses on the one hand on temporally dissociating the two by finding a TMS time window that differentially affects them and on the other hand on TMS-induced blindsight, which essentially reflects a difference in their respective effect size. Visual awareness is annihilated by TMS, while stimulus-related behavioral performance remains possible to some extent. Future research should aim to investigate the relationship between these two phenomena in order to integrate both in a general theory on the role of early visual cortex in visual awareness. 


\section{ACKNOWLEDGMENTS}

This study was supported by a grant to A.T.S. from the Dutch Organization for Scientific Research (NWO; grant number 400-07-048 and 452-06-003). We thank our medical supervisor Cees van Leeuwen, and our independent physician Martin van Boxtel. We also thank Annette Giani for her assistance with the psychophysical testing of the visual stimuli. 


\section{REFERENCES}

Amassian, V. E., Cracco, R. Q., Maccabee, P. J., Cracco, J. B., Rudell, A., \& Eberle, L. (1989). Suppression of visual perception by magnetic coil stimulation of human occipital cortex. Electroencephalogr Clin Neurophysiol, 74(6), 458-462.

Beckers, G., \& Homberg, V. (1991). Impairment of visual perception and visual short term memory scanning by transcranial magnetic stimulation of occipital cortex. Exp Brain Res, 87(2), 421432.

Beckers, G., \& Zeki, S. (1995). The consequences of inactivating areas V1 and V5 on visual motion perception. Brain, 118 ( Pt 1), 49-60.

Boyer, J. L., Harrison, S., \& Ro, T. (2005). Unconscious processing of orientation and color without primary visual cortex. Proc Natl Acad Sci U S A, 102(46), 16875-16879.

Busch, N. A., Dubois, J., \& VanRullen, R. (2009). The phase of ongoing EEG oscillations predicts visual perception. J Neurosci., 29(24), 7869-7876.

Cattaneo, Z., \& Silvanto, J. (2008a). Investigating visual motion perception using the transcranial magnetic stimulation-a daptation paradigm. Neuroreport, 19(14), 1423-1427.

Cattaneo, Z., \& Silvanto, J. (2008b). Time course of the state-dependent effect of transcranial magnetic stimulation in the TMS-adaptation paradigm. Neurosci Lett, 443(2), 82-85.

Christensen, M. S., Kristiansen, L., Rowe, J. B., \& Nielsen, J. B. (2008). Action-blindsight in healthy subjects after transcranial magnetic stimulation. Proc Natl Acad Sci U S A, 105(4), 1353-1357.

Corthout, E., Uttl, B., Juan, C. H., Hallett, M., \& Cowey, A. (2000). Suppression of vision by transcranial magnetic stimulation: a third mechanism. Neuroreport, 11(11), 2345-2349.

Corthout, E., Uttl, B., Walsh, V., Hallett, M., \& Cowey, A. (1999). Timing of activity in early visual cortex as revealed by transcranial magnetic stimulation. Neuroreport, 10(12), 2631-2634.

Corthout, E., Uttl, B., Ziemann, U., Cowey, A., \& Hallett, M. (1999). Two periods of processing in the (circum)striate visual cortex as revealed by transcranial magnetic stimulation. Neuropsychologia, 37(2), 137-145.

Cowey, A. (2008). TMS and visual awareness. In E. Wasserman (Ed.), Oxford handbook of transcranial stimulation. Oxford: Oxford University Press.

Crick, F., \& Koch, C. (1995). Are we aware of neural activity in primary visual cortex? Nature, 375(6527), 121-123.

Harris, J. A., Clifford, C. W., \& Miniussi, C. (2008). The functional effect of transcranial magnetic stimulation: signal suppression or neural noise generation? J Cogn Neurosci, 20(4), 734-740.

Haynes, J. D., \& Rees, G. (2005). Predicting the stream of consciousness from activity in human visual cortex. Curr Biol, 15(14), 1301-1307.

Kammer, T. (2007). Masking visual stimuli by transcranial magnetic stimulation. Psychol Res, 71(6), 659-666.

Kammer, T., Puls, K., Erb, M., \& Grodd, W. (2005). Transcranial magnetic stimulation in the visual system. II. Characterization of induced phosphenes and scotomas. Exp Brain Res, 160(1), 129140.

Koivisto, M., Mantyla, T., \& Silvanto, J. (2010). The role of early visual cortex (V1/V2) in conscious and unconscious visual perception. Neuroimage, 51(2), 828-834.

Koivisto, M., Railo, H., \& Salminen-Vaparanta, N. (2011). Transcranial magnetic stimulation of early visual cortex interferes with subjective visual awareness and objective forced-choice performance. Conscious Cogn, 20(2), 288-298.

Laycock, R., Crewther, D. P., Fitzgerald, P. B., \& Crewther, S. G. (2007). Evidence for fast signals and later processing in human V1/V2 and V5/MT+: A TMS study of motion perception. $J$ Neurophysiol, 98(3), 1253-1262.

Mathewson, K. E., Gratton, G., Fabiani, M., Beck, D. M., \& Ro, T. (2009). To see or not to see: prestimulus alpha phase predicts visual awareness. J Neurosci, 29(9), 2725-2732.

Muckli, L., Kohler, A., Kriegeskorte, N., \& Singer, W. (2005). Primary visual cortex activity along the apparent-motion trace reflects illusory perception. PLoS Biol, 3(8), e265.

Romei, V., Brodbeck, V., Michel, C., Amedi, A., Pascual-Leone, A., \& Thut, G. (2008). Spontaneous fluctuations in posterior alpha-band EEG activity reflect variability in excitability of human visual areas. Cereb Cortex, 18(9), 2010-2018.

Rosanova, M., Casali, A., Bellina, V., Resta, F., Mariotti, M., \& Massimini, M. (2009). Natural frequencies of human corticothalamic circuits. J Neurosci, 29(24), 7679-7685. 
Ruzzoli, M., Marzi, C. A., \& Miniussi, C. (2010). The neural mechanisms of the effects of transcranial magnetic stimulation on perception. J, 103(6), 2982-2989.

Sack, A. T., Kohler, A., Linden, D. E., Goebel, R., \& Muckli, L. (2006). The temporal characteristics of motion processing in hMT/V5+: combining fMRI and neuronavigated TMS. Neuroimage, 29(4), 1326-1335.

Sack, A. T., van der Mark, S., Schuhmann, T., Schwarzbach, J., \& Goebel, R. (2009). Symbolic action priming relies on intact neural transmission along the retino-geniculo-striate pathway. Neuroimage, 44(1), 284-293.

Silvanto, J., \& Muggleton, N. G. (2008). Testing the validity of the TMS state-dependency approach: targeting functionally distinct motion-selective neural populations in visual areas V1/V2 and V5/MT+. Neuroimage, 40(4), 1841-1848.

Silvanto, J., Muggleton, N. G., Cowey, A., \& Walsh, V. (2007). Neural adaptation reveals statedependent effects of transcranial magnetic stimulation. Eur J Neurosci, 25(6), 1874-1881.

Silvanto, J., \& Pascual-Leone, A. (2008). State-dependency of transcranial magnetic stimulation. Brain Topogr, 21(1), 1-10.

Skotte, J. H., Nojgaard, J. K., Jorgensen, L. V., Christensen, K. B., \& Sjogaard, G. (2007). Eye blink frequency during different computer tasks quantified by electrooculography. Eur J Appl Physiol, 99(2), 113-119.

Stevens, L. K., McGraw, P. V., Ledgeway, T., \& Schluppeck, D. (2009). Temporal characteristics of global motion processing revealed by transcranial magnetic stimulation. Eur J Neurosci, 30(12), 2415-2426.

Thielscher, A., Reichenbach, A., Ugurbil, K., \& Uludag, K. (2009). The cortical site of visual suppression by transcranial magnetic stimulation. Cereb Cortex, 20(2), 328-338.

Thut, G., Nietzel, A., Brandt, S. A., \& Pascual-Leone, A. (2006). Alpha-band electroencephalographic activity over occipital cortex indexes visuospatial attention bias and predicts visual target detection. J Neurosci, 26(37), 9494-9502.

Tong, F. (2003). Primary visual cortex and visual awareness. Nat Rev Neurosci, 4(3), 219-229.

van Dijk, H., Schoffelen, J. M., Oostenveld, R., \& Jensen, O. (2008). Prestimulus oscillatory activity in the alpha band predicts visual discrimination ability. J Neurosci., 28(8), 1816-1823.

VanderWerf, F., Brassinga, P., Reits, D., Aramideh, M., \& Ongerboer de Visser, B. (2003). Eyelid movements: behavioral studies of blinking in humans under different stimulus conditions. $J$ Neurophysiol, 89(5), 2784-2796.

Volkmann, F. C., Riggs, L. A., \& Moore, R. K. (1980). Eyeblinks and visual suppression. Science, 207(4433), 900-902.

Zeki, S., \& Bartels, A. (1998). The autonomy of the visual systems and the modularity of conscious vision. Philos Trans R Soc Lond B Biol Sci, 353(1377), 1911-1914. 


\section{Chapter 3 The temporal dynamics of early visual cortex involvement in behavioral priming}
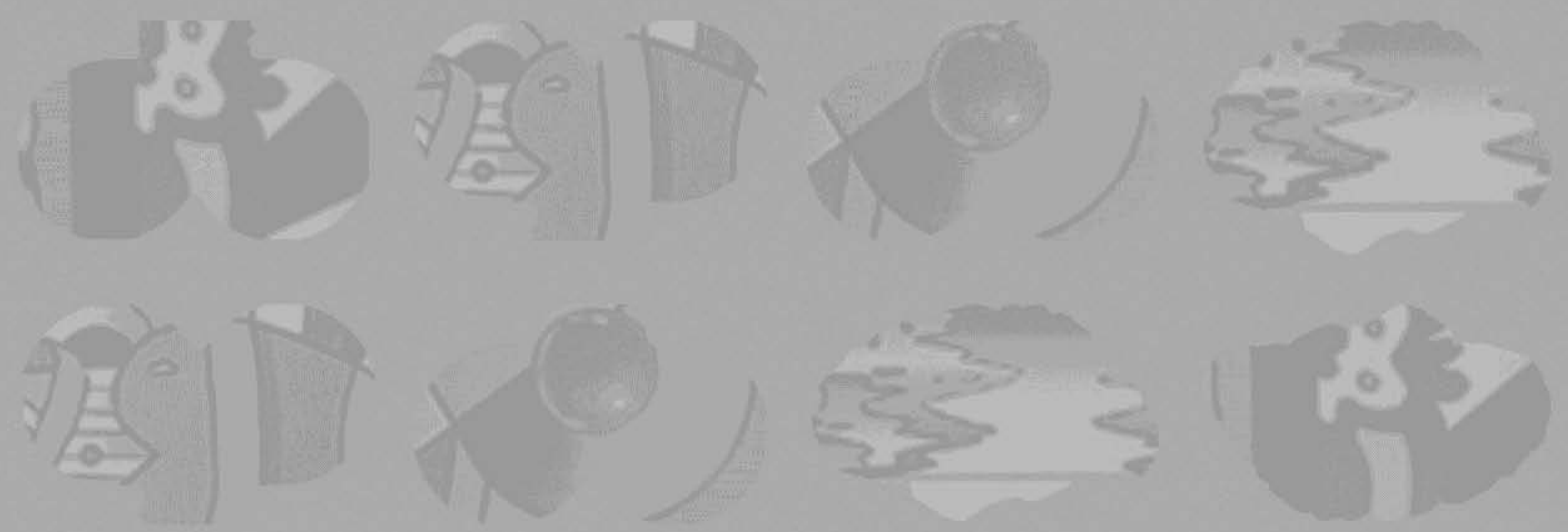

Based on: Jacobs, C, de Graaf, T:A, Goebel, R \& Sack, A.T. (2012) The temporal dynamics of early visual cortex involvement in behavioral priming, In revision

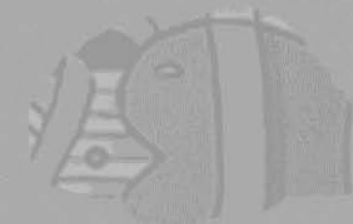




\section{ABSTRACT}

Subliminal priming studies have shown that the behavioral impact of a visual stimulus can be independent from its conscious perception. This dissociability of visual awareness and behavioral priming is likely mediated by two independent underlying neural signatures. Transcranial magnetic stimulation (TMS) allows for non-invasive interference with ongoing neural processing, and when applied in a chronometric design over early visual cortex (EVC), TMS has proved valuable in indicating at which particular time point EVC must remain unperturbed for conscious vision to be established. In the current study, we set out to examine the effect of chronometric EVC TMS across a broad range of time points, both before (pre-stimulus) and after (post-stimulus) the onset of symbolic visual stimuli in order to assess whether TMS-induced suppression of visual awareness can be dissociated from behavioral priming in the temporal domain. To this end, we evaluated chronometric TMS effects on three different measures of visual processing, namely performance on a standard visual discrimination task, a subjective rating of stimulus visibility, and a visual priming task. To control for non-neural TMS effects, we incorporated electrooculographical recordings, placebo TMS (sham), and control site TMS (vertex) in our design. Our results suggest that, when considering the appropriate control data, the temporal pattern of EVC TMS disruption on visual discrimination, subjective awareness and behavioral priming are not dissociable. Instead, our data suggest that unperturbed EVC activity at temporally specific time points is required for visual perception holistically - i.e. for visual discrimination, awareness, and priming -, and that this is the case both before and after the onset of a visual stimulus. The current findings are discussed in light of their implications on models of visual awareness and subliminal priming. 


\section{INTRODUCTION}

Subliminal perception refers to perception of a stimulus without accompanying conscious awareness. The neuropsychological phenomenon of blindsight is an example of subliminal perception (Weiskrantz, 2009), as blindsight patients notoriously claim not to experience vision in a part of their visual field, although they score above chance when asked to judge visual stimuli presented in their blind spot. Under experimental conditions it is possible to evaluate subliminal perception in healthy observers as well (Lau \& Passingham, 2006). The existence of subliminal perception raises the intriguing question to what extent the content of subliminal stimuli can steer later behavior, even if the perceiver does not report any conscious awareness of the stimulus. This issue has driven much psychophysical research on subliminal perception. Masked priming studies showed that a visual stimulus can indeed modulate subsequent behavior in the absence of visual awareness, and that this can occur at different levels of the visual hierarchy, from low-level color and form priming (Breitmeyer, Ogmen, \& Chen, 2004; Ro, Singhal, Breitmeyer, \& Garcia, 2009; Vorberg, Mattler, Heinecke, Schmidt, \& Schwarzbach, 2003) all the way up to semantic priming (Kouider \& Dehaene, 2009; Kouider, Dehaene, Jobert, \& Le Bihan, 2007; Van den Bussche, Notebaert, \& Reynvoet, 2009). The dissociation between awareness and behavioral influence, as apparent in all these psychophysical studies, triggered the search for the independent neural mechanisms underlying visual awareness and behavioral priming, which many suspect to involve early visual cortex (EVC; Breitmeyer, Ro, \& Ogmen, 2004; Koivisto, Henriksson, Revonsuo, \& Railo, 2012; Ro, 2008; Sack, van der Mark, Schuhmann, Schwarzbach, \& Goebel, 2009).

Since the pioneering work of Amassian in 1989 (Amassian et al., 1989), many researchers have reverted to chronometric Transcranial Magnetic Stimulation (TMS) in order to directly test the stimulus-locked functional relevance of early visual cortex ( i.e. V1, V2, V3) for visual perception over time. This elegant experimental approach allows for the spatially and temporally specific interference with regular neural processing in healthy human individuals, and all of these studies robustly showed that visual discrimination is impaired when a single TMS pulse is delivered to EVC approximately $90 \mathrm{~ms}$ after the onset of the visual stimulus (e.g. Amassian, et al., 1989; Beckers \& Homberg, 1991; Camprodon, Zohary, Brodbeck, \& Pascual-Leone, 2009; Corthout, Uttl, Juan, Hallett, \& Cowey, 2000; Corthout, Uttl, Walsh, Hallett, \& Cowey, 1999; Corthout, Uttl, Ziemann, Cowey, \& Hallett, 1999; de Graaf, Herring, \& Sack, 2011; Jolij \& Lamme, 2005; Lamme, 2006b; Laycock, Crewther, Fitzgerald, \& Crewther, 2007; Ro, 2008; Sack, et al., 2009). In other words, visual discrimination relies on intact EVC activity at $\sim 90 \mathrm{~ms}$ after the visual stimulus is presented.

Later studies included a direct, subjective measure of conscious perception in the form of self-reported awareness (de Graaf, Cornelsen, Jacobs, \& Sack, 2011; de Graaf, Goebel, \& Sack, 2011; de Graaf, Herring, et al., 2011; Koivisto, 
Mantyla, \& Silvanto, 2010; Koivisto, Railo, \& Salminen-Vaparanta, 2011; Overgaard, Nielsen, \& Fuglsang-Frederiksen, 2004), and showed that EVC TMS similarly affects subjective visual awareness and discrimination performance (de Graaf, Cornelsen, et al., 2011; de Graaf, Goebel, et al., 2011; Koivisto, et al., 2010; Koivisto, et al., 2011). Thus, visual processing in EVC 90ms after the presentation of a visual stimulus can be said to contribute to both visual discrimination and constitution of stimulus awareness, as reflected in both objective and subjective measures of visual perception.

Our group recently conducted a chronometric TMS study to investigate whether the visual suppression caused by disrupting EVC at the classical poststimulus time window leaves the behavioral impact of this stimulus, that had previously been shown not to require visual awareness (Vorberg, et al., 2003), on a second stimulus unaffected. The study revealed that both masking and priming functions break down when EVC TMS is applied $80-100 \mathrm{~ms}$ after the onset of the first visual stimulus in a combined masking-priming experiment (Sack, et al., 2009), suggesting that both discrimination performance and priming rely on intact processing in EVC around $90 \mathrm{~ms}$ post-stimulus onset. This conclusion was further corroborated by a study which examined priming of metacontrast masked stimuli after chronometric EVC TMS and also reported a reduction in priming $30-90 \mathrm{~ms}$ post-prime (Koivisto, Henriksson, Revonsuo, \& Railo, 2012). Even though the latter study had a slightly broader temporal scope $(30-180 \mathrm{~ms}$ post-stimulus), these findings do not rule out the possibility that a dissociation between visual awareness and behavioral priming might still exist in the temporal domain at other critical time periods of stimulus-related activity within EVC. This becomes particularly important when considering the recently established additional time windows of relevant EVC activity for visual processing, including time points later than $180 \mathrm{~ms}$ post-stimulus, and even time points prior to visual stimulus onset.

Concretely, in addition to the long established post-stimulus TMS time window of $\sim 90 \mathrm{~ms}$ for visual suppression, EVC TMS has been shown to interfere with visual discrimination performance at an additional time point around 200ms post-stimulus (Camprodon, et al., 2009; Volmer et al., 2011). Conscious perception has been repeatedly linked to feedback processing (Bullier, 2001; Koivisto, et al., 2011; Lamme \& Roelfsema, 2000; Pascual-Leone \& Walsh, 2001; Shulman, Hyder, \& Rothman, 2003), and the late effective TMS time window has been suggested to reflect recurrent processing in EVC (Camprodon, et al., 2009). Assuming that activity in EVC at this stage indeed dissociates between conscious and unconscious perception, subliminal priming should remain unaffected. To our knowledge, so far none of the studies employing chronometric EVC TMS in a (masked) priming paradigm was able to address this idea, generally not systematically testing such late TMS time windows for different measures of visual processing.

Another time frame, in which EVC TMS was found to disrupt normal visual perception, is a remarkable one, because it precedes the onset of the visual stimulus (Beckers \& Homberg, 1991; Corthout, et al., 2000; Corthout, Uttl, Zie- 
mann, et al., 1999; de Graaf, Cornelsen, et al., 2011; Jacobs, Goebel, \& Sack, 2012; Laycock, et al., 2007). The neural mechanisms underlying such a potential pre-stimulus EVC TMS suppression effect have to be fundamentally different from those underlying post-stimulus TMS. The post-stimulus TMS suppression effect has been claimed to probably exert its perceptual impairment by decreasing the signal-to-noise ratio exactly at that critical time period when the stimulus-related signal is being processed in EVC (Harris, Clifford, \& Miniussi, 2008; Silvanto \& Rees, 2011). However, at any pre-stimulus TMS time window affecting visual perception, the stimulus-related signal has not even reached EVC yet to be interfered with by TMS. Therefore, some of the few studies that revealed a pre-stimulus TMS visual suppression effect attributed this effect to TMS-induced eye blinking (Amassian et al., 1998; Corthout, et al., 2000; Sack, Kohler, Linden, Goebel, \& Muckli, 2006). Recently, however, we presented first empirical evidence that the suppressive influence of pre-stimulus TMS on visual perception remained present even after controlling for eye blinks or other non-specific TMS effects, suggesting that the prestimulus TMS effect on visual perception may after all be of neural origin (Jacobs, et al., 2012). This raises the question whether the pattern of behavioral consequences of pre-stimulus TMS is different to the pattern of behavioral consequences of post-stimulus EVC TMS, or in other words: are visual awareness and behavioral priming two dissociative neural processes in the pre-stimulus temporal domain?

The current study aimed to address these questions by testing whether EVC TMS at any pre- or post-stimulus time window selectively hinders the constitution of visual awareness while, e.g., leaving a potential subliminal behavioral priming effect intact, or whether it affects visual processing holistically, including objective recognition, subjective awareness, and behavioral priming. To this end, we went beyond previous work by employing a rigorous, systematic experimental paradigm covering an unprecedented range of TMS time windows, a wide range of behavioral measures, and multiple forms of TMS control. Thus, we measured both task performance and self-reported visual awareness in the context of a visual discrimination task, and we measured the behavioral impact of identical visual stimuli in a symbolic behavioral priming task. On each trial, a single TMS pulse was delivered over EVC at one of 20 different time points time-locked to the first visual stimulus and ranging from -80 to $300 \mathrm{~ms}$ in steps of 20ms. This extensive chronometric TMS design ensured to test several time windows post- as well as pre- visual stimulus onset. In addition, by broadening our temporal scope further into the post-stimulus domain than previous work, we could explore whether EVC selectively affects visual awareness at any time point beyond the classical TMS-induced masking time window of $80-100 \mathrm{~ms}$. Throughout the experimental sessions, electrooculographical (EoG) data were recorded to control for eye blinks. Additionally, in a separate sham TMS session, EVC was stimulated with a placebo TMS coil to account for the influence of TMS-related auditory stimulation. As a final control 
or non-specific TMS effects, a non-relevant site (vertex) was stimulated with genuine TMS using the identical chronometric design as applied over EVC.

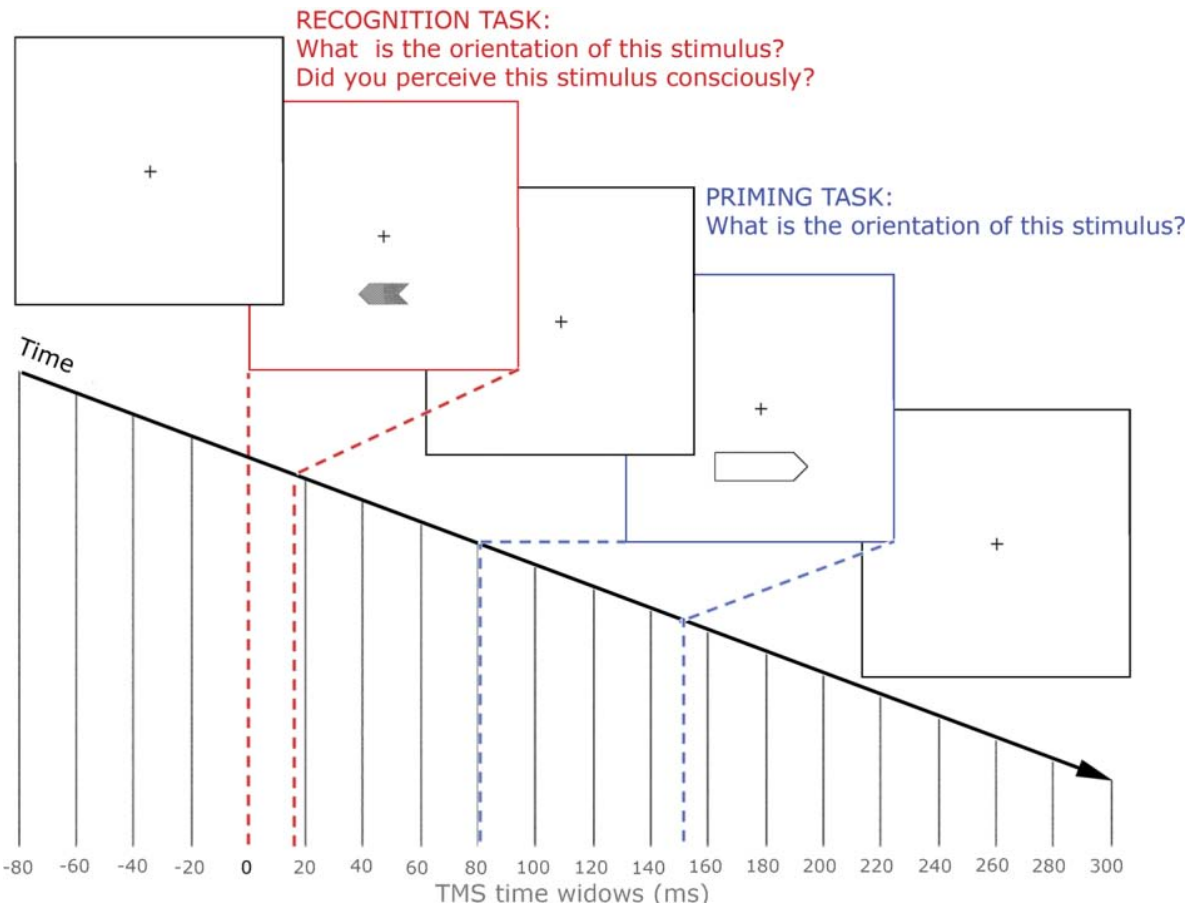

Figure 1. Trial time line. A horizontal black arrow (S1) was presented below fixation for $16.7 \mathrm{~ms}$, followed by a larger horizontal arrow (S2) for $66.7 \mathrm{~ms}$ with an SOA of $83.3 \mathrm{~ms}$. During each trial a single TMS pulse was delivered over EVC at any of 20 different time points time-locked to S1 (range from -80 to $200 \mathrm{~ms}$ in steps of $20 \mathrm{~ms}$ ). In the recognition task, participants were required to indicate via button press whether they had perceived the first arrow consciously or not. By a second button press they indicated whether they thought the first arrow pointed leftward or rightward. In the priming task, participants were required to indicate via button press whether they thought the second arrow pointed leftward or rightward.

\section{MATERIALS AND METHODS}

\section{Participants}

18 healthy participants (6 males, mean age $23.6 y$, range $19-32$ ) with normal or corrected-to-normal vision participated in this study, including one of the authors (CJ). 10 Participants received TMS at the experimental site EVC, whereas the other 8 received TMS at a control site (vertex). 
Three participants were excluded from further analysis (two in main experiment and one in the vertex control experiment), when their baseline accuracy in the recognition task proved to be below 75\% correct responses.

The study was approved by the Medical Ethics committee of the University Medical Center, Maastricht, the Netherlands. Prior to the experiment all participants were requested to fill out a medical questionnaire, which was screened and approved by a medical supervisor. At the start of each experimental session, participants filled out an additional questionnaire to check whether current circumstances allowed TMS application. All participants gave written informed consent at the start of each session and were compensated financially for their participation.

\section{Stimuli}

Two schematic drawings of horizontal arrows on a white background served as stimuli. They were presented serially on each trial of the recognition as well as the priming task, and were located in the horizontal center of the lower visual field, $0.8^{\circ}$ visual angle below fixation. The first stimulus (S1) was a small $\left(0.8^{\circ}\right.$ by $1.86^{\circ}$ ) arrow presented for $16.7 \mathrm{~ms}$. It was followed by a larger arrow stimulus (S2) which consisted of black outer contours only, and was on screen for $66.7 \mathrm{~ms}$. Both arrow stimuli pointed in either leftward or rightward direction and their relative stimulus-onset asynchrony was fixed at $83.3 \mathrm{~ms}$. Stimulus presentation and recording of behavioral responses was accomplished through the Presentation software package (Neurobehavioral Systems, Inc., Albany, CA).

Stimuli were shown on a 17" TFT, Samsung SyncMaster 931 DF computer monitor with a refresh rate of $60 \mathrm{~Hz}$. Calibration measurements using a photodiode on the monitor and concurrent measurement of the external TMS triggering signal leaving the parallel port of the stimulus pc showed a constant, stable and reliable offset of $2 \mathrm{~ms}$ for all stimulus-TMS SOAs. When we discuss SOAs in the remainder of the paper, we refer to the SOAs as requested of our presentation software.

\section{Experiment}

A within-subject design was employed in which participants performed two different two alternative forced-choice (2AFC) visual discrimination tasks. The complete study consisted of four experimental sessions: in two out of four sessions, the recognition task was performed; in the other two, the priming task was performed. The study asked for an extensive dataset, because of the large amount of TMS time windows under investigation here. To prevent participant fatigue, data collection was therefore spread over four two-hour sessions per participant. Session order was counterbalanced across participants. All stimulus and TMS parameters were identical for both tasks, only task instruction 
differed. At the start of each session, participants were comfortably seated in front of the monitor, and their heads were stabilized in a chin-rest.

In the recognition task, participants covertly paid attention to the direction of the first symbolic arrow, S1. They were instructed to retain fixation throughout the experiment and to be as accurate as possible in their responses. To measure their subjective visual awareness, they were asked to indicate via button press whether they consciously perceived S1 or not. All participants were instructed to respond positively to this question when their percept entailed some informational content, even if they would still be unsure on stimulus direction. By their second key press, participants indicated which direction, i.e. left or right, they believed S1 pointed in. The task was set up as a 2AFC, so it was not possible for participants to continue the experiment unless two responses were given on each trial. Participants were explicitly informed that the two responses were independent, i.e. indicating that $\mathrm{S} 1$ was consciously perceived did not mean that a correct answer on its direction was required.

Participants were trained on the task until they reached an average accuracy of at least $80 \%$ correct responses on three consecutive blocks of 20 trials with a maximum of 15 blocks. If the accuracy threshold was not reached within these 15 blocks, the participant was not included in the study. At single trial level, performance was indicated by the fixation cross, which would turn green after correct responses and red after incorrect responses. During breaks, summary feedback was given to the participants about their performance on the previous block.

Each session included a No TMS baseline measurement. This measurement consisted of 20 trials during which no feedback was given and no TMS pulses were delivered. Participants then completed 400 TMS trials, divided over 20 blocks. On each of these trials a single TMS pulse was randomly delivered at one of 20 TMS time windows ranging from -80 to $300 \mathrm{~ms}$ post $\mathrm{S} 1$-onset in $20 \mathrm{~ms}$ steps (see Figure 1). Inter-trial-interval was jittered and had an average duration of $5000 \mathrm{~ms}$. In each session 20 trials per time window were collected leading to a total number of 40 trials per time window.

In the behavioral priming task participants responded to the direction of $\mathrm{S} 2$, via button press and as quickly as possible. In this task, the main goal was to measure the behavioral priming effect of S1 on the reaction times (RTs) to S2. RTs to $\mathrm{S} 2$ are expected to be shorter in case S1 pointed in the identical direction (i.e. congruent trial) than when it pointed in opposite direction (i.e. incongruent trial). No measure of subjective awareness was implemented here since visual awareness of S2 was not deemed relevant to the research question. No training was offered to participants, since the task was fairly easy and our dependent variable in this task does not depend on any prior training. 


\section{TMS protocol}

Phosphene localization was used to determine coil position. The initial position of the TMS coil was approximately $1 \mathrm{~cm}$ above the inion. While the participants were fixating, they received single pulses of TMS. Participants reported whether they perceived TMS-induced phosphenes, and if so, where these were located within their visual field. Coil position was then systematically varied until the induced phosphenes overlapped with the visual field location of the experimental stimuli. If complete overlap could not be achieved, the coil position was chosen at which induced phosphenes were closest to the desired location. TMS applied over stimulation sites based on phosphene localization have been shown to most strongly target V2/V3 (Salminen-Vaparanta, Noreika, Revonsuo, Koivisto, \& Vanni, 2011; Thielscher, Reichenbach, Ugurbil, \& Uludag, 2009).

During each trial a single biphasic TMS pulse was administered at any of 20 TMS time windows. TMS time windows were all time-locked to S1 ranging from -80 to $300 \mathrm{~ms}$ post-S1 onset in $20 \mathrm{~ms}$ steps. The stimulation intensity was fixed across participants and set to $70 \%$ of maximal stimulator output (Medtronic Functional Diagnostics A/S, Skovlunde, Denmark; maximum stimulator output $=1.9 \mathrm{~T}$ ). Given this intensity of stimulation, any visual suppression effects are unlikely to originate from visual masking through TMS-induced phosphenes, because phosphene thresholds usually are far below 70\% maximum output for the used stimulator type. Phosphene thresholds in a subset of our participants were 51\% maximum stimulator output on average. An increase of the intensity above this threshold (as in our case) has been shown not to result in an increased visibility of phosphenes, but in a transition of a TMS-induced phosphene into a TMS-induced masking effect (Kammer, Puls, Erb, \& Grodd, 2005; Kastner, Demmer, \& Ziemann, 1998). All pulses were administered with a figure-of-eight coil (MC-B70, the inner and outer radii of the two coil loops are 1.2 and $5.4 \mathrm{~cm}$, respectively). The TMS coil was positioned horizontally, with the coil handle pointing rightwards.

\section{Control measures for non-neural TMS effects}

TMS is known to also induce non-neural side-effects due to the confounding acoustical and sensorimotor stimulation inherent to any TMS protocol. Therefore, we included a number of complementary control measures (de Graaf \& Sack, 2011) to account for such possible non-neural effects of TMS on our visual awareness and behavioral priming measures.

First, EoG data were recorded to collect information on the participants' vertical eye movements. Trials containing an eye blink in the time interval -200 to $100 \mathrm{~ms}$ relative to $\mathrm{S} 1$ onset were labeled 'blink' trials, and excluded from the subsequent analyses on time-specific TMS-induced masking or priming (for a detailed description and comparison of the data before and after eye blinks 
removal and their putative role in pre-stimulus masking, please consult Chapter 2 Materials and Methods section or Jacobs, et al., 2012).

Second, to control for the clicking sound accompanying TMS pulses, data of the experimental sessions were compared to data acquired in a sham TMS session using a specific TMS placebo coil (MC-P-B70 Placebo) with 6 participants of the original sample. The sham TMS pulse was randomly delivered at any of the original pre-stimulus time windows (i.e. $-80,-60,-40,-20$, and $0 \mathrm{~ms}$ ). The measured time windows were limited to the pre-stimulus domain, because it would be too strenuous on the participants to undergo another four sessions of data acquisition, especially since we anyway expected the auditory click to have its biggest alerting influence when it precedes the visual stimulus.

Third, to also control for the aversive sensations on the head resulting from magnetic stimulation of the scalp that the placebo TMS coil does not mimic, we decided to re-run the entire experiment with a different group of subjects, this time stimulating vertex as control site with real TMS instead of EVC. Vertex was defined as $\mathrm{Cz}$ in the 10-20 electrode positioning system.

For direct comparison to the EVC experimental data, acquiring the vertex data in the original participant sample would have been optimal. However, because we failed to find all original participants able and willing to invest in the study for another four sessions, and since we were cautious for a lack of motivation in participants when having to perform too many task repetitions, we decided to acquire the vertex data in a new participant sample as a betweensubject factor.

\section{Analyses}

On the basis of the EoG data, trials were classified as 'blink' or 'no blink' trials. Trials were considered blink trials if an eye blink was detected anytime between $-200 \mathrm{~ms}$ prior and $100 \mathrm{~ms}$ post S1-onset (for details on the analysis of the EoG data, see Skotte, Nojgaard, Jorgensen, Christensen, \& Sjogaard, 2007). After analyses of the complete EVC TMS dataset, the eye blink trials were removed, and the analyses were repeated for residual data.

In the recognition task, we measured both subjective visual awareness and recognition accuracy. Subjective visual awareness was indicated by participants on a two-point scale, corresponding to whether they regarded S1 as 'seen' or 'unseen'. The percentage of 'seen' responses was then calculated per condition, i.e. per TMS time window. In a similar fashion, the percentage of correctly identified trials per time window served as our measure of accuracy. We calculated these values for each subject individually and performed one-way repeatedmeasures analysis-of-variance (RM-ANOVA) of the factor Time Window separately on both dependent variables. Post-hoc analyses comprised of comparisons of all 20 experimental levels of the factor Time Window to the baseline condition No TMS. The alpha values for these pairwise comparisons were least-square difference (LSD) corrected. 
In the behavioral priming task, reaction times (RTs) were taken as a dependent variable. Outlier analysis consisted of excluding those trials on which RTs exceeded the 1.5xIQR criterion per participant. Only RTs of correct trials were analyzed. Average RTs were calculated for each TMS time window and for congruent (Con) and incongruent (INCon) trials separately. The average RTs on congruent trials were subtracted from the average RTs on incongruent trials resulting in a measure of behavioral priming which we termed the priming effect (PE). PE scores were calculated for each TMS time window in each individual. On the priming data a two-way RM-ANOVA was conducted with factors Time Window (21 levels) and Congruency (2 levels). The factor Congruency was then collapsed into the single measure of the PE and PE data was submitted to a one-way RM-ANOVA with Time Window as the single factor. Again, post-hoc analyses consisted of comparisons of all 20 experimental levels of the factor Time Window to the baseline condition No TMS, and alpha values for these pair wise comparisons were LSD corrected.

The data of the vertex control experiment were analyzed in the same way as described for the experimental data. To compare the vertex and EVC TMS data a two-way mixed ANOVA was performed with Time Window (21 levels) as within-subject factor and Site (2 levels) as between-subjects factor. Significant interactions were further investigated by means of independent samples t-test comparing the group data per time window.

For the recognition part of the sham TMS session, average accuracy and awareness scores per participant and per sham TMS time window were calculated. These two measures served as input for a two-way RM-ANOVA with factor TMS Type (2 levels: Sham versus EVC TMS) and Time Window (21 levels), followed by a one-way RM-ANOVA on the sham data with single factor Time Window. For the priming part of the sham TMS session, PEs were calculated per participant and per sham time window (see above). Again, a two-way RMANOVA including factors Type and Time Window and a one-way RM-ANOVA on the sham TMS data were then conducted. Post hoc analyses consisted of pair wise comparisons of the 5 sham TMS time windows to No TMS baseline with LSD-corrected alphas.

For all ANOVAs conducted, the Huyn-Feldt correction for degrees of freedom was applied, if equal variances could not be assumed. 


\section{RESULTS}

\section{Recognition task}

\section{Accuracy}

The one-way RM-ANOVA showed a main effect of TMS Time Window on the average percentage correct responses $(\mathrm{F}(8.244,49.463)=4.083, \mathrm{p}=.001)$. Post hoc analysis comparing all TMS time windows to baseline revealed that recognition accuracy dropped significantly compared to No TMS (mean $=91.9$ $\%$ correct) when a TMS pulse was delivered at 80 (mean $=70.0 \%$ correct, $\mathrm{p}=$ .009 ) or $100 \mathrm{~ms}$ (mean $=71.2 \%$ correct, $\mathrm{p}=.01$ ) after S1 onset, and at -80 (mean $=77.5 \%$ correct, $\mathrm{p}=.042)$ or $-60 \mathrm{~ms}($ mean $=77.5 \%, \mathrm{p}=.023)$ prior to $\mathrm{S} 1$ onset (see Chapter 2, Figure 3.A).

In contrast to the time-specific effects of EVC TMS, sham TMS using a placebo TMS coil did not have a significant effect of Time Window on accuracy scores $(\mathrm{F}(1.360,6.798)=2.009 ; \mathrm{p}=.197)$. This was confirmed by the significant interaction effect $(\mathrm{F}(5,25)=7.986 ; \mathrm{p}<.001)$ between TMS Type and Time Window as was found in the conjoined two-way RM-ANOVA on the sham and EVC data (see Chapter 2, Figure 5.A and Jacobs, et al., 2012).

The two-way mixed ANOVA demonstrated a main effect of Time Window $(\mathrm{F}(4.92,59.09)=4.36 ; \mathrm{p}<.001)$ and a significant interaction effect of TMS Time Window x Site (EVC vs. vertex) $(\mathrm{F}(4.92,59.09)=4.65 ; \mathrm{p}<.001)$. Independent samples t-tests comparing EVC to vertex groups for each time window revealed that accuracy differed between groups when TMS was applied $80 \mathrm{~ms}(\mathrm{p}=.01)$, $100 \mathrm{~ms}(\mathrm{p}=.01)$ and $120 \mathrm{~ms}(\mathrm{p}=.03)$ post-stimulus. In the pre-stimulus domain a significant effect of stimulation site was apparent in the $-60 \mathrm{~ms}$ time window $(p=.03)$. Furthermore, trends were observed $-40 \mathrm{~ms}(\mathrm{p}=.06)$ and $-80 \mathrm{~ms}(\mathrm{p}=$ .07) pre-stimulus time windows (see Figure 2.A).

The one-way RM-ANOVA on the vertex stimulation data in isolation revealed a significant effect of Time Window $(\mathrm{F}(14.268,85.607)=1.906, \mathrm{p}=$ .036), but post hoc comparisons did not show significant changes in accuracy of TMS applied at any tested time window compared to baseline (see Chapter 2, Figure 5.A).

Together, these results clearly indicate that time-specific pre- and a poststimulus TMS pulses applied to EVC (and not vertex) lead to reduced visual discrimination ability. 

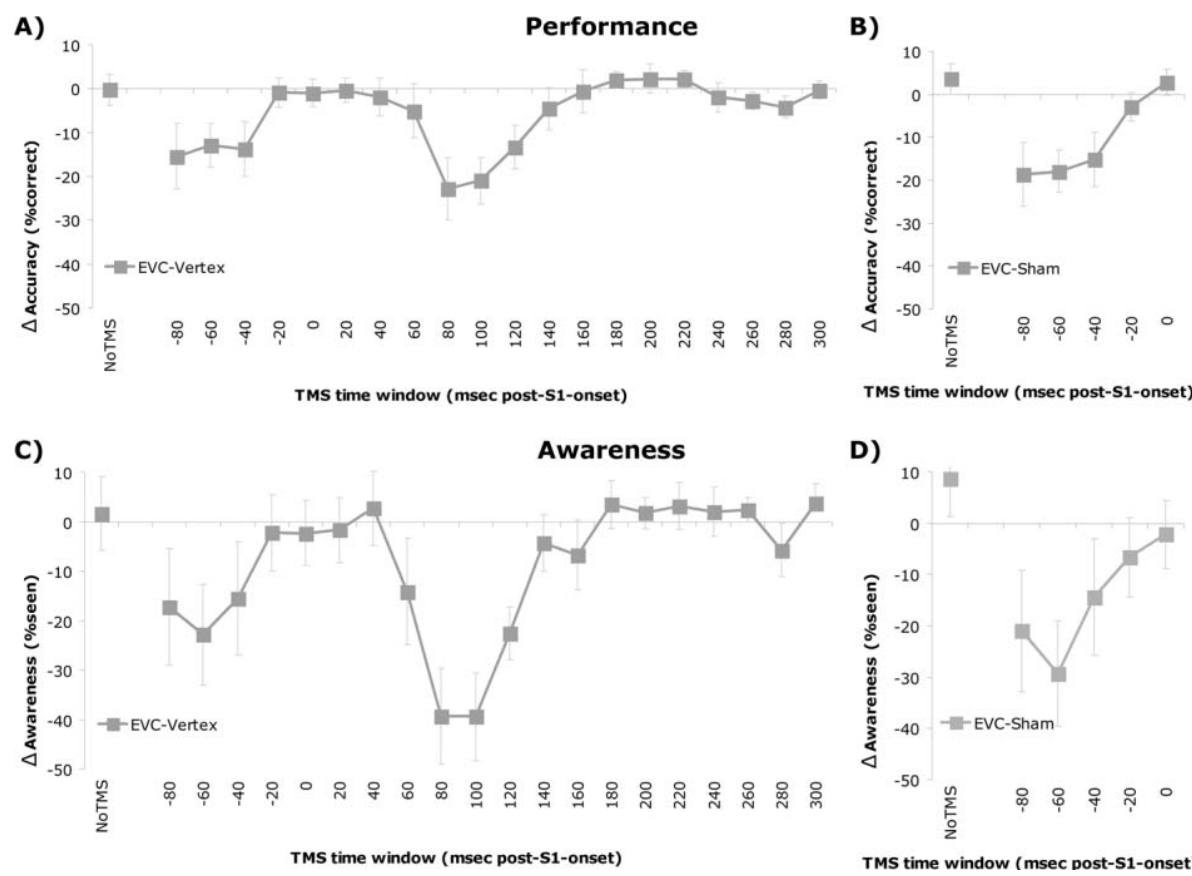

TMS time window (msec post-S1-onset]

Figure 2. Recognition task data. A) Difference scores between accuracies, defined as the percentage correct responses, in the EVC TMS sessions versus the average accuracy in the vertex TMS sessions per TMS time window. Error bars represent standard errors of the mean (SEM). B) Difference scores between accuracies, defined as the percentage correct responses, in the EVC TMS sessions versus the average accuracy in the sham TMS session per TMS time window. Error bars represent SEM. C) Difference scores between awareness, defined as the percentage of trials indicated as seen, in the EVC TMS sessions versus the average awareness in the vertex TMS sessions per TMS time window. Error bars represent SEM. D) Difference scores between awareness, defined as the percentage of trials indicated as seen, in the EVC TMS sessions versus the average awareness in the sham TMS session per TMS time window. Error bars represent SEM.

\section{Self-reported awareness}

As on accuracy, TMS time window had an effect on subjective awareness rating $(\mathrm{F}(5.166,30.9995)=4.525, \mathrm{p}=.003)$. Compared to No $\mathrm{TMS}($ mean $=84.9 \%$ seen), awareness was significantly reduced when EVC TMS was applied at -60 ms pre-stimulus (mean $=55.7 \%$ seen, $\mathrm{p}=.043)$, and 80 (mean $=41.5 \%$ seen, $\mathrm{p}$ $=.006), 100$ (mean $=42.7 \%$ seen, $\mathrm{p}=.005)$, and $120 \mathrm{~ms}$ (mean $=58.8 \%$ seen, $\mathrm{p}$ $=.023$ ) post-stimulus time windows (see Chapter 2, Figure 3.B and Jacobs et al., 2012).

Both sham TMS $(\mathrm{F}(5,25)=2.009 ; \mathrm{p}=.112)$, as well as vertex TMS $(\mathrm{F}(5.503$, $33.015)=1.249, \mathrm{p}=.308$ ) did not lead to a significant effect of Time Window on self-reported visual awareness (see Chapter 2, Figure 5.B). Again, the two-way 
RM-ANOVA revealed a significant interaction effect $(F(5,25)=3.440 ; p=.017)$ of Type and Time Window (see Figure 2.D).

As was the case for the accuracy data, the mixed ANOVA demonstrated a main effect of Time Window $(\mathrm{F}(5.03,60.31)=3.93 ; \mathrm{p}<.001)$ and a Time Window x Site interaction effect $(\mathrm{F}(5.03,60.31)=4.23 ; \mathrm{p}<.001)$. Post-hoc analyses showed that TMS applied at $80 \mathrm{~ms}(\mathrm{p}=.01), 100 \mathrm{~ms}(\mathrm{p}<.001)$ and 120 $\mathrm{ms}(\mathrm{p}=.02)$ post-stimulus affected the experimental and the control group differently, and a trend towards the same effect was observed for $-60 \mathrm{~ms}(\mathrm{p}=.08)$ TMS (see Figure 2.C).

\section{Behavioral priming task}

During the preprocessing stages, $6.2 \%$ blink trials, $4.5 \%$ outliers and $3.8 \%$ incorrect responses were removed from the dataset. The two-way RM-ANOVA conducted on the cleaned EVC data revealed a significant main effect of Congruency $(\mathrm{F}(1,7)=284.43, \mathrm{p}<.001)$, with higher estimated average reaction times for incongruent $($ mean $=408.85 \mathrm{~ms}$ ) than for congruent trials $($ mean $=$ $372.5 \mathrm{~ms}$ ). Moreover, a significant interaction between the factors Congruency and Time Window $(\mathrm{F}(5.42,37.94)=2.97, \mathrm{p}=.02)$ (see Figure 3.A) was revealed.

The subsequent one-way RM-ANOVA on the PE including post hoc comparisons of the different levels of Time Window was performed to quantify the effect of chronometric TMS on the behavioral impact of the stimulus. Reflecting the dependence of the prime's behavioral impact on timing of the TMS pulse, as was already demonstrated by the interaction effect in the two-way RM-ANOVA, this analysis showed a main effect of Time Window $(\mathrm{F}(5.42,37.94)=2.97, \mathrm{p}=$ .02)

Post hoc analyses demonstrated that the priming effect decreased significantly compared to No TMS (mean PE $=45.5 \mathrm{~ms}(\mathrm{Con}=360.7 \mathrm{~ms}$; $\mathrm{INCon}=406.2$ $\mathrm{ms}$ )) when single-pulse TMS was applied $60 \mathrm{~ms}$ (mean PE $=11.1 \mathrm{~ms}(\mathrm{Con}=$ $386.6 \mathrm{~ms}$; INCon $=397.7 \mathrm{~ms}), \mathrm{p}=.039), 80 \mathrm{~ms}$ (mean PE $=8.0 \mathrm{~ms}(\mathrm{Con}=385.3 \mathrm{~ms}$; INCon $=393.3 \mathrm{~ms}$ ), $\mathrm{p}=.041), 100 \mathrm{~ms}$ (mean PE $=11.0 \mathrm{~ms}$ (Con $=379.0 \mathrm{~ms}$; INCon $=390.0 \mathrm{~ms}), \mathrm{p}=.011)$ and $280 \mathrm{~ms}($ mean PE $=17.9 \mathrm{~ms}$ (Con= $385.5 \mathrm{~ms}$; INCon $=$ $403.4 \mathrm{~ms}), \mathrm{p}=.030$ ) post $\mathrm{S} 1$-onset. The pre-stimulus time windows at which we find visual suppression in the recognition task, did not lead to any significant decrease in priming effect (time window $-80 \mathrm{~ms}: \mathrm{p}=.91$; time window $-60: \mathrm{p}=$ .33).

The two-way RM-ANOVA with factors Type and Time Window showed a main effect of Time Window $(\mathrm{F}(5,25)=2.808 ; \mathrm{p}=.038)$, as well as a significant interaction between both factors $(F(5,25)=5.459 ; \mathrm{p}=.002)$.

However, the sham TMS data did not show an effect of Time Window on priming $(F(5,25)=1.332, p=.283)$, as shown in the one-way RM-ANOVA, suggesting that sham TMS did not affect priming in a temporally specific fashion, or in comparison to baseline performance (see Figure 3.B). 

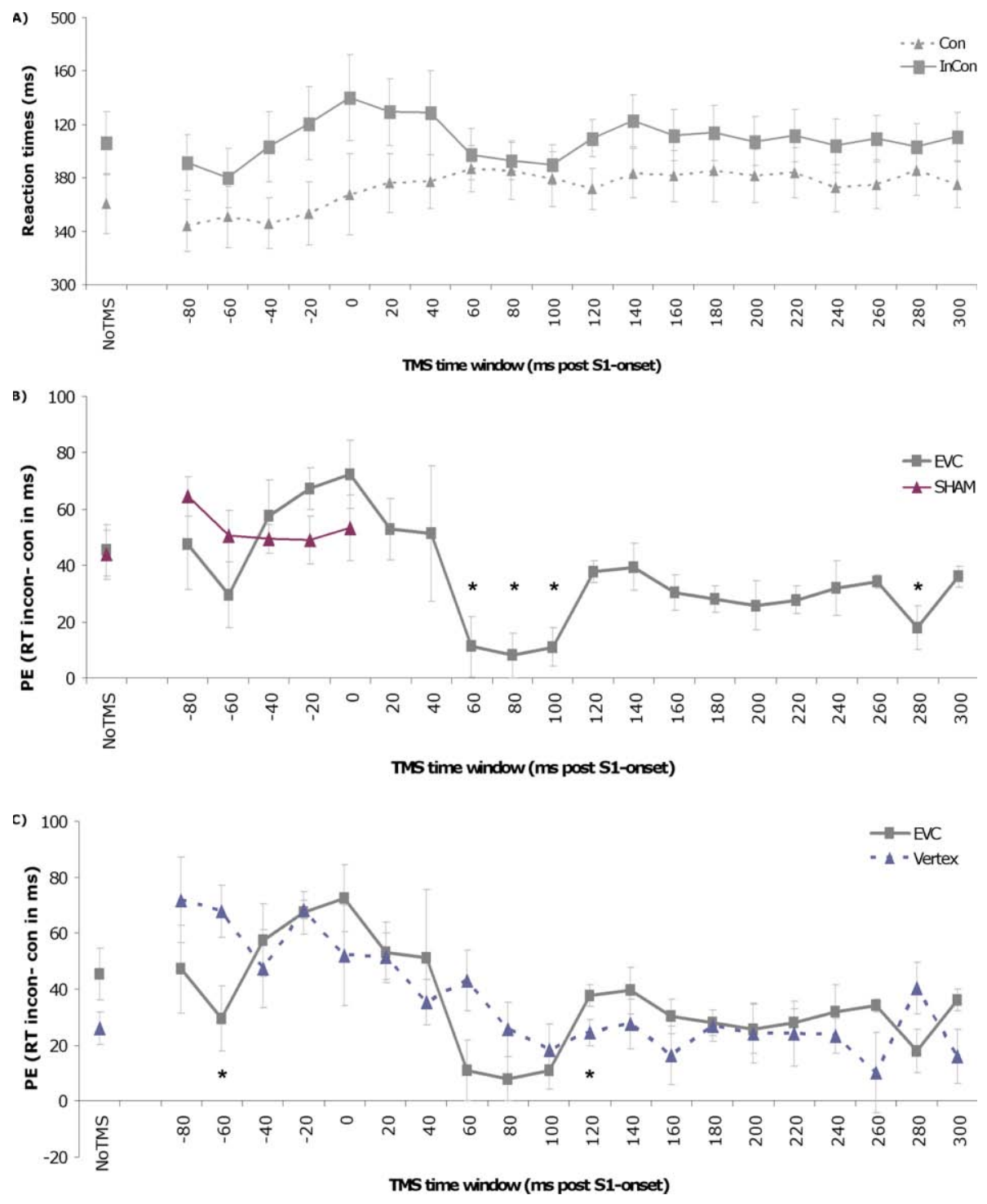

Figure 3. Behavioral priming data. A) Reaction times (RT) to congruent (solid line) and incongruent (dashed line) S2 stimuli per TMS time window. Only experimental (i.e. EVC) data are depicted here. Error bars represent standard errors of the mean (SEM). B) The average priming effect (PE), defined as the reaction times (RTs) in milliseconds on incongruent trials minus the RTs on congruent trials, across TMS time windows is plotted for both the EVC TMS data (grey line) and the placebo (sham) TMS data (purple line). Error bars represent standard errors of the mean. Significant differences relative to No TMS are indicated with an asterisk. C) The average priming effect (PE), defined as the reaction times (RTs) in milliseconds on incongruent trials minus the RTs on congruent trials, across TMS time windows for both the EVC and vertex TMS data. Significant differences between the two groups are indicated with an asterisk. 


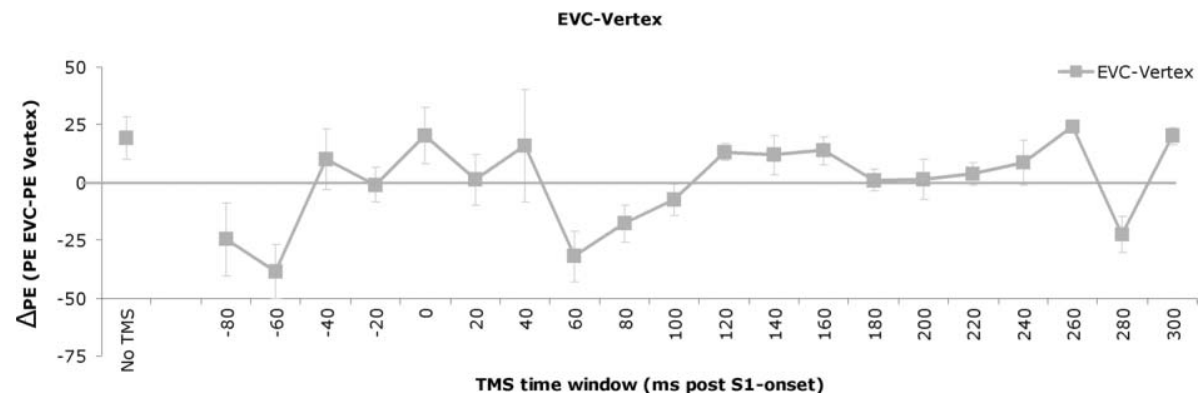

Figure 4. Estimation of the net neural TMS effect. Average priming effect (PE) in the EVC TMS experiment per TMS time window after subtraction of the average PE across participants in the vertex TMS experiment. Error bars represent standard errors of the mean (SEM).

The two-way mixed ANOVA with Time Window as within-subject factor and Site as between-subject factor showed a significant main effect of Time Window and a trend towards an interaction effect of Time Window $\times$ Site $(\mathrm{F}(8.83,114.78)=$ 1.88; $\mathrm{p}=.063)$. The independent samples t-tests comparing the EVC and vertex data per time window revealed a group difference for the $120 \mathrm{~ms}(\mathrm{p}=.05)$ poststimulus and $-60 \mathrm{~ms}(\mathrm{p}=.03)$ pre-stimulus time windows, and trends for the $60 \mathrm{~ms}(\mathrm{p}=.06), 280 \mathrm{~ms}(\mathrm{p}=.08)$ and $300 \mathrm{~ms}(\mathrm{p}=.06)$ post-stimulus time windows. These data indicate that the trend towards interaction is not solely driven by the different in post-stimulus PE, but also by the smaller pre-stimulus (-60ms) PE in the EVC versus the vertex group.

This set of findings now seemed contradictory: the EVC results in themselves did not show any pre-stimulus TMS effect on PE, yet when taking into account the vertex control data the group analysis suggested a pre-stimulus TMS effect on PE after all. The situation is resolved by considering the vertex data in isolation: the factor Time Window proved to affect behavioral priming $(\mathrm{F}(14.25,85.49)=5.12, \mathrm{p}<.001)$. Post hoc comparisons revealed an increase in priming compared to baseline (mean $\mathrm{PE}=26.0 \mathrm{~ms}$ ) for the pre-stimulus time period, which reached significance for the $-80 \mathrm{~ms}$ (mean $\mathrm{PE}=71.93 \mathrm{~ms}, \mathrm{p}=$ .011 ), $-60 \mathrm{~ms}$ (mean PE $=67.82 \mathrm{~ms}, \mathrm{p}=.001$ ), and $-20 \mathrm{~ms}$ (mean PE $=68.36 \mathrm{~ms}, \mathrm{p}$ $<.001$ ) time windows. The $20 \mathrm{~ms}$ (mean $\mathrm{PE}=48.4 \mathrm{~ms}, \mathrm{p}=.061$ ) and $60 \mathrm{~ms}$ (mean $\mathrm{PE}=40.6 \mathrm{~ms}, \mathrm{p}=.07$ ) post-stimulus time windows demonstrated a trend in the same direction (see Figure 3.C). Together, these results indicate that prestimulus and early post-stimulus TMS over vertex lead to an enhancement of behavioral priming. As vertex was chosen as an irrelevant control site, we presume that this enhancement is a consequence of non-neural side effects of TMS stimulation.

This finding thus suggests an interesting situation: the non-neural effects of TMS at pre-stimulus windows seem to have an enhancing effect on PE (as shown by increased PEs by vertex stimulation). Yet, this enhancing effect is not apparent in the EVC condition, thus likely counteracted by the neural suppres- 
sive effects of pre-stimulus TMS over occipital cortex. This would suggest that pre-stimulus TMS over EVC indeed suppresses the priming effect (analogous to its suppressive effects on recognition accuracy and visual awareness), but that this effect was counteracted by the enhancing non-neural effects of TMS pulses. To visualize the suggested veridical time course of neural TMS effects on PE, we subtracted the EVC results from the vertex results in Figure 4.

\section{DISCUSSION}

The current study employed an extensive chronometric TMS design including several complementary control measures in order to reveal the temporal pattern of early visual cortex (EVC) involvement in conscious visual perception and its relation to symbolic behavioral priming. To this end, single pulses of EVC TMS stimulation at 20 time points ranging from -80 to $300 \mathrm{~ms}$ poststimulus onset were applied in steps of $20 \mathrm{~ms}$ while participants performed two different two alternative forced choice (2AFC) tasks, i.e. visual discrimination and behavioral priming tasks, assessing objective discrimination, subjective awareness, and behavioral priming of visual stimuli. After controlling for eye blinks, EVC TMS led to a diminished discrimination performance, when applied 80 and $100 \mathrm{~ms}$ post-stimulus onset, and when applied -60 and $-80 \mathrm{~ms}$ prior to stimulus onset. Self-reported visual awareness was impaired by 80, 100 and $120 \mathrm{~ms}$ post-stimulus TMS, and by $-60 \mathrm{~ms}$ pre-stimulus TMS. Behavioral priming was affected by 80 and $100 \mathrm{~ms}$ post-stimulus TMS but at first glance not by prestimulus TMS. However, the vertex TMS data (in contrast to the sham TMS data), representing both the acoustic and sensorimotor side effects of TMS, also showed a time-specific effect on behavioral priming. Priming was increased by vertex TMS, particularly when the TMS pulse preceded the prime stimulus. Compared to vertex TMS, EVC TMS diminished priming, also in the pre-stimulus domain.

\section{Post-stimulus effects of EVC TMS on visual perception}

Taken together, we here revealed two distinct time periods at which an intact EVC is necessary for the generation of visual awareness. First, TMS successfully masks the visual stimulus when applied at the time windows $80-120$ ms poststimulus onset, a result in line with the vast amount of studies that already established EVC's role in visual processing within this time period (e.g Amassian, et al., 1989; Beckers \& Homberg, 1991; Camprodon, et al., 2009; Corthout, et al., 2000; Corthout, Uttl, Walsh, et al., 1999; Corthout, Uttl, Ziemann, et al., 1999; de Graaf, Herring, et al., 2011; Jolij \& Lamme, 2005; Lamme, 2006b; Laycock, et al., 2007; Ro, 2008; Sack, et al., 2009). Both objective and subjective measures of visual awareness, i.e. task performance and selfreported awareness, respectively, captured this perceptual deficit, as both 
dependent variables suffered when the TMS pulse followed the visual stimulus by $80-100 \mathrm{~ms}$. Behavioral priming is also affected by EVC TMS when applied 60 , $80,100 \mathrm{~ms}$ post-stimulus onset, indicating decreased impact of the visual stimulus on subsequent behavior, and suggesting that at least for the post-stimulus temporal domain, behavioral priming and visual awareness are not dissociable within EVC. This finding is in excellent accordance with our and other's previous findings on the interdependence of awareness and priming at poststimulus EVC TMS time windows (Koivisto, et al., 2012; Sack, et al., 2009).

In addition to our previous findings, our design now enabled us to broaden our temporal scope further into the post-stimulus domain. The later TMS time window of visual suppression, potentially reflecting feedback from higher (extrastriate) cortical areas was not replicated in this study. Therefore, the hypothesized dissociation of priming and visual awareness was not found for post-stimulus timings later than the classical masking time window. We did reveal an unexpected effect on priming at $280 \mathrm{~ms}$ post-stimulus onset. However, we believe that this very late time window of $280 \mathrm{~ms}$ reflects an effect TMS had on the perception of $\mathrm{S} 2$, the target stimulus in the priming task, because it is specific to the priming task and does not reveal itself in the recognition task at all.

From psychophysical studies, we know the concept of subliminal priming, i.e. a stimulus that does not cross the threshold for conscious awareness can still influence subsequent behavior, which can be instantiated through visual masking (Eimer \& Schlaghecken, 2003; Ogmen, Breitmeyer, \& Melvin, 2003; Schacter \& Buckner, 1998; Vorberg, et al., 2003). In this light, it is striking that no such dissociation appears in our data. There are theoretical reasons to expect the two processes to be dissociable in the temporal domain.

First, based on the idea that the recurrent activity looping between striate and extrastriate areas is a prerequisite for visual awareness, at least two poststimulus time windows would be predicted to show visual awareness impairment; an early one that reflects the interruption of the feedforward stream and a later one that reflects the prevention of feedback. Behavioral priming also depends on an intact geniculo-striate pathway, as we have demonstrated in an earlier study (Sack, et al., 2009). But, because priming does not require a conscious percept of the prime stimulus, this process should only rely on EVC at the early, feedforward stage. Although data from both functions overlap at 80$100 \mathrm{~ms}$ post-stimulus onset, we do not find a late time window at which TMS selectively interferes with visual awareness. (Note that on the basis of these data we cannot exclude the possibility that the priming task relies on both conscious and unconscious prime perception and that we only interfered with the conscious component by EVC TMS, while leaving unconscious processing of the prime stimulus intact.)

Second, the fast, transient magnocellular visual pathway has been suggested to carry sufficient information for behavioral priming, but visual awareness does not arise until the slow, sustained parvocellular activity has reached the visual cortex (Paulus, Korinth, Wischer, \& Tergau, 1999). Visual masking ex- 
ploits the different dynamics of these two pathways, and occurs when the prime-mask SOA is chosen such that, through interchannel connections, the magnocellular (M) activity related to the mask can suppress the parvocellular (P) activity belonging to the prime stimulus (Breitmeyer and Ogmen, 2000, Breitmeyer et al., 2004, Ogmen et al., 2003). Accordingly, EVC TMS would affect behavioral priming and visual awareness at two distinct functional time points. Indeed, we find a shift in the critical time windows of $20 \mathrm{~ms}$ as behavioral priming was affected by EVC TMS $60-100 \mathrm{~ms}$ after prime onset, and visual awareness after $80-120 \mathrm{~ms}$. The transient nature of the magnocellular pathway would however predict a narrower time span of priming reduction.

In a recent study on masked priming with stimuli of varied contrast, Tapia and Breitmeyer (2011) showed that the size of the priming effect resembled the contrast-response function of M-neurons during conscious vision, and the contrast-response function of P-neurons during unconscious vision. Based on these results, the authors put forward a model that assigns the magnocellular pathway a critical role in conscious vision: it projects to the dorsal visual processing stream and pre-frontal cortex, which in turn potentiate the stimulusrelated recurrent activity instantiated in the ventral visual stream by the activation of the parvocellular channel. They propose that the feedforward activity in the parvocellular pathway and cortical ventral stream pathways might cause the behavioral priming effect. This would mean that visual awareness and priming both rely on processing in the parvocellular stream. The model would concretely predict that the functional relevance of EVC for behavioral priming and visual awareness overlap, at the moment when EVC receives the slow parvocellular input. Moreover, the temporal window at which EVC TMS is detrimental should be broader in the case of visual awareness, because this process depends on both the early input of the faster magnocellular channel, and on the late recurrent activity in the ventral visual stream.

Our results indicate that EVC is indeed functionally relevant for both priming and visual awareness at overlapping post-stimulus time points. However, this interval is not broader for visual awareness compared to priming, and we did not find a second, discrete time period at which only visual awareness is hindered. On the other hand, we found the time period of TMSinduced impairment to be shifted $20 \mathrm{~ms}$ forward for subjective awareness, running from 80 to $120 \mathrm{~ms}$ instead of the 60 to $100 \mathrm{~ms}$ post-stimulus onset at which priming is impaired. These data seem to best fit the model of Koivisto et al. (2011) that considers the last phase of the subjective awareness effect, when task performance is no longer hindered by EVC TMS, as representative of local recurrent activity responsible for the generation of visual awareness (Koivisto, et al., 2011; Koivisto \& Silvanto, 2011). The idea that recurrent processing in EVC is a precondition for visual awareness has been raised before (Bullier, 2001; Lamme, 2001; Lamme \& Roelfsema, 2000; Tong, 2003), but it was suggested to consist of backprojections from distant cortical areas (Lamme, 2006a) feeding the information back to EVC at a later time point, namely around $200 \mathrm{~ms}$ 
post-stimulus (Camprodon, et al., 2009). As we do not find a second poststimulus time window of TMS-induced masking around this latency, but we do find a dissociation between behavioral priming and visual awareness for the $120 \mathrm{~ms}$ post-stimulus TMS time window, our data seem to render support for local recurrent activity as a prerequisite for visual awareness. Prime awareness is most probably not required for behavioral priming, so the impairment is not hindered by EVC TMS around $120 \mathrm{~ms}$, when it interferes with local recurrent processes. Nevertheless, this does still not explain why TMS already affects behavioral priming $60 \mathrm{~ms}$ after prime onset, a time point at which EVC apparently does not play a role for visual awareness yet.

\section{Pre-stimulus effects of EVC TMS on visual perception}

The second time period of successful TMS-induced masking occurred when the magnetic pulse preceded the visual stimulus by 40 to $80 \mathrm{~ms}$. This pre-stimulus TMS effect is much less established, and though it has been reported in previous studies (Beckers \& Homberg, 1991; Corthout, et al., 2000; Corthout, Uttl, Ziemann, et al., 1999; de Graaf, Cornelsen, et al., 2011; Jacobs, et al., 2012; Laycock, et al., 2007), it remains controversial. Its neural origin has particularly been called into question. Recently, we have provided evidence against nonneural accounts by showing that eye blinking, multisensory enhancement, or heightened levels of attention do not suffice to explain the pre-stimulus TMS effect (see Chapter 2 or Jacobs, et al., 2012), and we thus concluded that the pre-stimulus TMS effect likely has a neural basis in early visual cortex.

The skepticism regarding the neural nature of pre-stimulus TMS was probably inspired by the fact that a TMS pulse hindering the perception of a not-yetpresented stimulus is very counterintuitive. In fact, immediate signal suppression, the proposed post-stimulus TMS mechanism of action (Harris, et al., 2008), cannot underlie pre-stimulus TMS effects, since there is no cortical signal to suppress yet. However, because alpha power has been shown to correlate negatively with visual awareness (Mathewson, Gratton, Fabiani, Beck, \& Ro, 2009; Thut, Nietzel, Brandt, \& Pascual-Leone, 2006; Toscani, Marzi, Righi, Viggiano, \& Baldassi, 2010; van Dijk, Schoffelen, Oostenveld, \& Jensen, 2008), and the phase of ongoing alpha oscillations was shown to predict whether or not a visual stimulus reaches awareness, we have proposed that a pre-stimulus TMS pulse might evoke neuronal activity in the alpha frequency band $(\sim 10 \mathrm{~Hz})$, that is unfavorable to later visual input (de Graaf, Cornelsen, et al., 2011; Jacobs, et al., 2012). The absence of any suppressive TMS effects in the vertex or sham data for the pre- and post-stimulus domains provide further evidence for the notion that both the pre- and post-stimulus TMS-induced masking effect reflect neural mechanisms in EVC (see also Jacobs, et al., 2012).

At first glance, a pre-stimulus dissociation between awareness and behavioral priming seemed to be revealed, in the sense that pre-stimulus TMS selectively interfered with visual awareness and objective discrimination at -60 
ms prior to visual stimulus onset, whereas behavioral priming remained unaffected. The current data seemed to indicate that this behavioral pattern can be achieved by TMS-induced masking. Concretely, this would mean that the processing routes of priming and visual awareness could be made to diverge by interfering with EVC prior to prime onset. Possibly, the brain state evoked by pre-stimulus TMS still allowed some sort of shallow processing of the stimulus, which might suffice for priming. Visual awareness might require a deeper form of processing, and would therefore be more susceptible to the dominant, disturbing influence of the evoked brain state. The sham control data fitted nicely into this line of reasoning, because they did not show any effects of TMS time window, and we could thus conclude that the revealed pre-stimulus dissociation is not linked to the auditory stimulation that comes with the TMS pulse. However, after controlling for the non-neural TMS effects of sensorimotor stimulation using vertex TMS, the priming data of the pre-stimulus time windows showed to be systematically affected in terms of a vertex TMSinduced increase of the priming effect. This non-neural TMS enhancement of priming is mixed with its neural EVC TMS suppression effect leading to an absence of priming decrease in time window -80 and $-60 \mathrm{~ms}$. Because the TMS effects in the experimental data were composed of a non-neural and a neural process counteracting each other, we estimated the size of the neural component by subtracting the vertex data for visualization purposes in Figure 4. This revealed decreased behavioral priming in the -80 and $-60 \mathrm{~ms}$ pre-stimulus TMS time windows, hence, the same time windows at which visual awareness is impaired. In other words, after taking the vertex data into consideration, we conclude that both visual awareness and behavioral priming rely on intact EVC at the exact same time periods, both pre- and post-stimulus.

\section{Methodological considerations}

Aside from these conceptual implications of our findings, we would like to also stress an interesting and remarkable methodological outcome of our study: as reported in the current study, we revealed that TMS over vertex resulted in a time-specific increase, i.e. enhancement, of the priming effect of S1 on S2. Interestingly, this baseline increase due to pre-stimulus vertex TMS proved to be task-specific. This means, while vertex TMS time-specifically enhanced the priming effect of S1 on S2, it did not affect recognition accuracy or subjective awareness of S1. The lack of any effect on recognition accuracy at any of the tested vertex TMS time windows cannot be ascribed to a ceiling effect, because baseline accuracy was not perfect (i.e. $\sim 90 \%$ correct on average), leaving room for potential improvement.

We suggest that the vulnerability of the tasks to attentional modulation might differ. In the priming task, participants were explicitly instructed to pay attention to, and respond to, S2. Because S1 did not carry any task-relevant information ( $50 \%$ of trials were congruent, $50 \%$ were incongruent), we can 
safely assume that they did not direct attention towards S1 in any top-down fashion. Any cue drawing bottom-up attention to S1, such as the TMS pulse, could therefore have a significant beneficial influence on stimulus processing. The elevated level of attention in the early TMS time windows due to the alerting TMS pulse indeed caused a baseline increase in priming, reflecting a bigger behavioral impact of S1 on S2. In the recognition task, participants were already attentive towards S1, since they were required to respond to it. Thus, less gain was to be expected from the non-neural alerting aspects of the TMS pulse, and, in accordance with this expectation, accuracy did not improve.

Sham TMS does not have the attention drawing effect that we see in the vertex data, which asks for a comparison of the two types of TMS control. Both sham and vertex TMS share the clicking sound generated in the (placebo) coil. We can rule out the auditory stimulation per se as the source of the prestimulus alerting effect, because the effect does not show in the sham TMS data. Sham TMS and vertex data differ when it comes to the sensory stimulation of the scalp, which is present in real TMS, and therefore also in control site TMS, but which is absent in the case of sham stimulation. The mildly aversive somatosensory experience, rather than the auditory experience, appears to be responsible for the alerting effect present in the pre-stimulus vertex data.

Generalizing, we conclude that if the task under investigation requires little top-down attention, the alerting influence of skull sensations accompanying pre-stimulus (or even post-stimulus) TMS is higher, and consequently, extra care should be taken when choosing the appropriate control conditions.

\section{ACKNOWLEDGEMENTS}

The research leading to these results has received funding from the Netherlands Organization for Scientific Research (NWO; grant number 400-07048 to A.T.S. and 021-002-087 to T.A.G.), and the European Research Council under the European Union's Seventh Framework Programme (FP7/20072013) / ERC Grant agreement $n^{\circ}$ [263472]).

We thank our medical supervisor Cees van Leeuwen, and our independent physician Martin van Boxtel. We also thank Annette Giani for her assistance with the psychophysical testing of the visual stimuli and Jeroen Mulders for assistance during data acquisition. 


\section{REFERENCES}

Amassian, V. E., Cracco, R. Q., Maccabee, P. J., Cracco, J. B., Rudell, A., \& Eberle, L. (1989). Suppression of visual perception by magnetic coil stimulation of human occipital cortex. Electroencephalogr Clin Neurophysiol, 74(6), 458-462.

Amassian, V. E., Cracco, R. Q., Maccabee, P. J., Cracco, J. B., Rudell, A. P., \& Eberle, L. (1998). Transcranial magnetic stimulation in study of the visual pathway. [Review]. Journal of clinical neurophysiology : official publication of the American Electroencephalographic Society, 15(4), 288-304.

Beckers, G., \& Homberg, V. (1991). Impairment of visual perception and visual short term memory scanning by transcranial magnetic stimulation of occipital cortex. Exp Brain Res, 87(2), 421432.

Breitmeyer, B. G., Ogmen, H., \& Chen, J. (2004). Unconscious priming by color and form: different processes and levels. Conscious Cogn., 13(1), 138-157.

Breitmeyer, B. G., Ro, T., \& Ogmen, H. (2004). A comparison of masking by visual and transcranial magnetic stimulation: implications for the study of conscious and unconscious visual processing. Conscious Cogn., 13(4), 829-843.

Bullier, J. (2001). Feedback connections and conscious vision. Trends Cogn Sci, 5(9), 369-370.

Camprodon, J. A., Zohary, E., Brodbeck, V., \& Pascual-Leone, A. (2009). Two phases of V1 activity for visual recognition of natural images. J Cogn Neurosci, 22(6), 1262-1269.

Corthout, E., Uttl, B., Juan, C. H., Hallett, M., \& Cowey, A. (2000). Suppression of vision by transcranial magnetic stimulation: a third mechanism. Neuroreport, 11(11), 2345-2349.

Corthout, E., Uttl, B., Walsh, V., Hallett, M., \& Cowey, A. (1999). Timing of activity in early visual cortex as revealed by transcranial magnetic stimulation. Neuroreport, 10(12), 2631-2634.

Corthout, E., Uttl, B., Ziemann, U., Cowey, A., \& Hallett, M. (1999). Two periods of processing in the (circum)striate visual cortex as revealed by transcranial magnetic stimulation. Neuropsychologia, 37(2), 137-145.

de Graaf, T. A., Cornelsen, S., Jacobs, C., \& Sack, A. T. (2011). TMS effects on subjective and objective measures of vision: Stimulation intensity and pre- versus post-stimulus masking. Conscious Cogn, 20[4], 1244-1255.

de Graaf, T. A., Goebel, R., \& Sack, A. T. (2011). Feedforward and quick recurrent processes in early visual cortex revealed by TMS? Neuroimage, 61[3], 651-659.

de Graaf, T. A., Herring, J., \& Sack, A. T. (2011). A chronometric exploration of high-resolution 'sensitive TMS masking' effects on subjective and objective measures of vision. Exp Brain Res, 209(1), 19-27.

de Graaf, T. A., \& Sack, A. T. (2011). Null results in TMS: from absence of evidence to evidence of absence. Neurosci Biobehav Rev, 35(3), 871-877.

Eimer, M., \& Schlaghecken, F. (2003). Response facilitation and inhibition in subliminal priming. Biol Psychol, 64(1-2), 7-26.

Harris, J. A., Clifford, C. W., \& Miniussi, C. (2008). The functional effect of transcranial magnetic stimulation: signal suppression or neural noise generation? J Cogn Neurosci, 20(4), 734-740.

Jacobs, C., Goebel, R., \& Sack, A. T. (2012). Visual awareness suppression by pre-stimulus brain stimulation; a neural effect. Neuroimage, 59(1), 616-624.

Jolij, J., \& Lamme, V. A. (2005). Repression of unconscious information by conscious processing: evidence from affective blindsight induced by transcranial magnetic stimulation. Proc Natl Acad Sci U S A, 102(30), 10747-10751.

Kammer, T., Puls, K., Erb, M., \& Grodd, W. (2005). Transcranial magnetic stimulation in the visual system. II. Characterization of induced phosphenes and scotomas. Exp Brain Res, 160(1), 129 140.

Kastner, S., Demmer, I., \& Ziemann, U. (1998). Transient visual field defects induced by transcranial magnetic stimulation over human occipital pole. Exp Brain Res, 118(1), 19-26.

Koivisto, M., Henriksson, L., Revonsuo, A., \& Railo, H. (2012). Unconscious response priming by shape depends on geniculostriate visual projection. EurJ Neurosci, 35(4), 623-633.

Koivisto, M., Mantyla, T., \& Silvanto, J. (2010). The role of early visual cortex (V1/V2) in conscious and unconscious visual perception. Neuroimage, 51(2), 828-834. 
Koivisto, M., Railo, H., \& Salminen-Vaparanta, N. (2011). Transcranial magnetic stimulation of early visual cortex interferes with subjective visual awareness and objective forced-choice performance. Conscious Cogn, 20(2), 288-298.

Koivisto, M., \& Silvanto, J. (2011). Relationship between visual binding, reentry and awareness. Conscious Cogn.

Kouider, S., \& Dehaene, S. (2009). Subliminal number priming within and across the visual and auditory modalities. Exp Psychol., 56(6), 418-433.

Kouider, S., Dehaene, S., Jobert, A., \& Le Bihan, D. (2007). Cerebral bases of subliminal and supraliminal priming during reading. Cereb Cortex, 17(9), 2019-2029.

Lamme, V. A. (2001). Blindsight: the role of feedforward and feedback corticocortical connections. Acta Psychol (Amst). 107(1-3), 209-228.

Lamme, V. A. (2006a). Towards a true neural stance on consciousness. Trends Cogn Sci, 10(11), 494501.

Lamme, V. A. (2006b). Zap! Magnetic tricks on conscious and unconscious vision. Trends Cogn Sci, 10(5), 193-195.

Lamme, V. A., \& Roelfsema, P. R. (2000). The distinct modes of vision offered by feedforward and recurrent processing. Trends Neurosci, 23(11), 571-579.

Lau, H. C., \& Passingham, R. E. (2006). Relative blindsight in normal observers and the neural correlate of visual consciousness. Proc Natl Acad Sci U S A, 103(49), 18763-18768.

Laycock, R., Crewther, D. P., Fitzgerald, P. B., \& Crewther, S. G. (2007). Evidence for fast signals and later processing in human V1/V2 and V5/MT+: A TMS study of motion perception. $J$ Neurophysiol, 98(3), 1253-1262.

Mathewson, K. E., Gratton, G., Fabiani, M., Beck, D. M., \& Ro, T. (2009). To see or not to see: prestimulus alpha phase predicts visual awareness. J Neurosci, 29(9), 2725-2732.

Ogmen, H., Breitmeyer, B. G., \& Melvin, R. (2003). The what and where in visual masking. Vision Res, 43(12), 1337-1350.

Overgaard, M., Nielsen, J. F., \& Fuglsang-Frederiksen, A. (2004). A TMS study of the ventral projections from V1 with implications for the finding of neural correlates of consciousness. Brain Cogn, 54(1), 58-64.

Pascual-Leone, A., \& Walsh, V. (2001). Fast backprojections from the motion to the primary visual area necessary for visual awareness. Science, 292(5516), 510-512.

Paulus, W., Korinth, S., Wischer, S., \& Tergau, F. (1999). Differential inhibition of chromatic and achromatic perception by transcranial magnetic stimulation of the human visual cortex. Neuroreport., $10(6), 1245-1248$.

Ro, T. (2008). Unconscious vision in action. Neuropsychologia, 46(1), 379-383.

Ro, T., Singhal, N. S., Breitmeyer, B. G., \& Garcia, J. O. (2009). Unconscious processing of color and form in metacontrast masking. Atten Percept Psychophys., 71(1), 95-103.

Sack, A. T., Kohler, A., Linden, D. E., Goebel, R., \& Muckli, L. (2006). The temporal characteristics of motion processing in hMT/V5+: combining fMRI and neuronavigated TMS. Neuroimage, 29(4), 1326-1335.

Sack, A. T., van der Mark, S., Schuhmann, T., Schwarzbach, J., \& Goebel, R. (2009). Symbolic action priming relies on intact neural transmission along the retino-geniculo-striate pathway. Neuroimage, 44(1), 284-293.

Salminen-Vaparanta, N., Noreika, V., Revonsuo, A., Koivisto, M., \& Vanni, S. (2011). Is selective primary visual cortex stimulation achievable with TMS? Hum Brain Mapp, 33[3], 652-665.

Schacter, D. L., \& Buckner, R. L. (1998). Priming and the brain. Neuron, 20(2), 185-195.

Shulman, R. G., Hyder, F., \& Rothman, D. L. (2003). Cerebral metabolism and consciousness. C R Biol, 326(3), 253-273.

Silvanto, J., \& Rees, G. (2011). What does Neural Plasticity Tell us about Role of Primary Visual Cortex (V1) in Visual Awareness? Front Psychol, 2, 6.

Skotte, J. H., Nojgaard, J. K., Jorgensen, L. V., Christensen, K. B., \& Sjogaard, G. (2007). Eye blink frequency during different computer tasks quantified by electrooculography. Eur J Appl Physiol, 99(2), 113-119.

Tapia, E., \& Breitmeyer, B. G. (2011). Visual consciousness revisited: magnocellular and parvocellular contributions to conscious and nonconscious vision. Psychol Sci, 22(7), 934-942.

Thielscher, A., Reichenbach, A., Ugurbil, K., \& Uludag, K. (2009). The cortical site of visual suppression by transcranial magnetic stimulation. Cereb Cortex, 20(2), 328-338. 
Thut, G., Nietzel, A., Brandt, S. A., \& Pascual-Leone, A. (2006). Alpha-band electroencephalographic activity over occipital cortex indexes visuospatial attention bias and predicts visual target detection. J Neurosci, 26(37), 9494-9502.

Tong, F. (2003). Primary visual cortex and visual awareness. Nat Rev Neurosci, 4(3), 219-229.

Toscani, M., Marzi, T., Righi, S., Viggiano, M. P., \& Baldassi, S. (2010). Alpha waves: a neural signature of visual suppression. Exp Brain Res, 207[3-4], 213-219.

Van den Bussche, E., Notebaert, K., \& Reynvoet, B. (2009). Masked primes can be genuinely semantically processed: a picture prime study. Exp Psychol., 56(5), 295-300.

van Dijk, H., Schoffelen, J. M., Oostenveld, R., \& Jensen, O. (2008). Prestimulus oscillatory activity in the alpha band predicts visual discrimination ability. J Neurosci., 28(8), 1816-1823.

Vorberg, D., Mattler, U., Heinecke, A., Schmidt, T., \& Schwarzbach, J. (2003). Different time courses for visual perception and action priming. Proc Natl Acad Sci U S A, 100(10), 6275-6280.

Weiskrantz, L. (2009). Blindsight: a case study spanning 35 years and new developments (3rd ed.). Oxford Oxford University Press. 



\section{Chapter 4 Topographic contribution of early visual cortex to short-term memory consolidation}

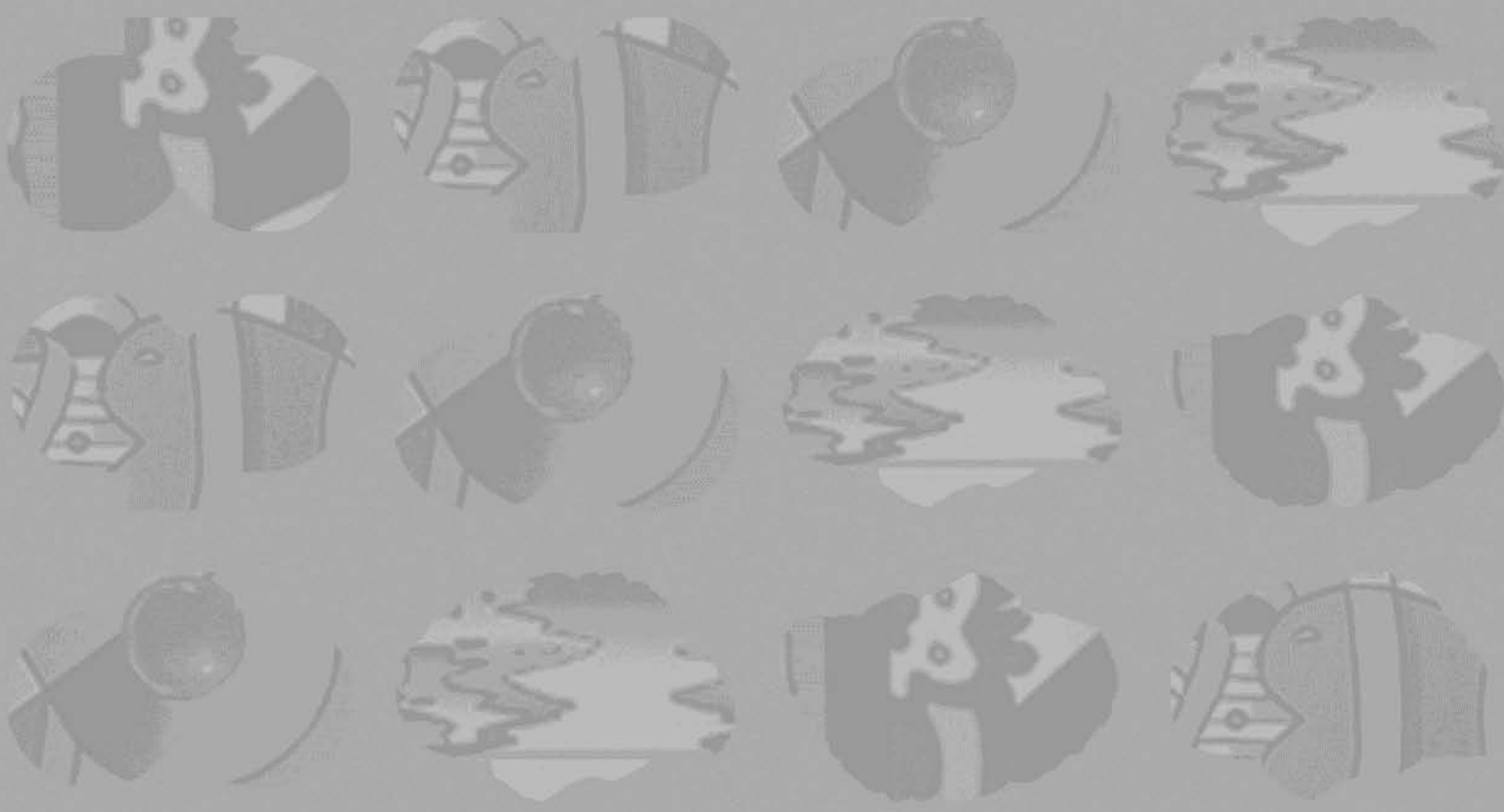

Based on: Van de Ven, V., Jacobs, C. \& Sack, A.T. (2012)

Topographic contribution of early visual cortex to short-term memory consolidation: a transcranial magnetic stimulation study, Journal of Neuroscience, 32(1), 4-11. 


\section{ABSTRACT}

The neural correlates for retention of visual information in VSTM are considered separate from those of sensory encoding. However, recent findings suggest that sensory areas may play a role also in short-term memory. We investigated the functional relevance, spatial specificity and temporal characteristics of human early visual cortex in the consolidation of capacity-limited topographic visual memory using transcranial magnetic stimulation (TMS). Topographically-specific TMS pulses were delivered over lateralized occipital cortex at 100,200 or $400 \mathrm{~ms}$ into the retention phase of a modified change detection task with low or high memory loads. We found decreased memory performance for memory trials in the visual field contralateral, but not ipsilateral to the side of TMS when pulses were delivered at $200 \mathrm{~ms}$ into the retention interval, for the high but not the low memory load. A behavioral version of the TMS experiment, in which a distractor stimulus (memory mask) replaced the TMS pulses, further corroborated these findings. Our findings suggest that retinotopic visual cortex contributes to the short-term consolidation of topographic visual memory at early stages of the retention of visual information. Further, TMS-induced interference decreased the strength or amplitude of the memory representation, which most strongly affected the high memory load trials. 


\section{INTRODUCTION}

The visual short-term memory (VSTM) system effectively retains visual sensory information well after the sensory stimulation has ended. Short-term retention may include the consolidation of visual information into the neural system, in which the information can be manipulated and used to guide our future behavior (Jonides, et al., 2008). Neurophysiological and brain imaging studies suggest that the neural correlates of VSTM are separate from those that encode the sensory information (Courtney, Ungerleider, Keil, \& Haxby, 1997; D'Esposito, Postle, \& Rypma, 2000; Goldman-Rakic, 1995; Linden, et al., 2003; Miller, Erickson, \& Desimone, 1996; Munk, et al., 2002). However, there is increasing evidence that sensory cortex may play an important role in short-term memory (Fuster, 1995; Pasternak \& Greenlee, 2005). Several animal neurophysiology studies reported increased activity in higher-level (Fuster \& Jervey, 1982; Miyashita \& Chang, 1988), as well as low-level visual areas (Super, Spekreijse, \& Lamme, 2001) during visual short-term retention. Further, while most functional magnetic resonance imaging (fMRI) studies of VSTM did not reveal increased activity in sensory brain areas during retention (Courtney, et al., 1997; Linden, et al., 2003; Munk, et al., 2002), recent functional magnetic resonance imaging (fMRI) studies showed that visual cortex may contribute to retention of visual information in other ways than increased activity (Harrison \& Tong, 2009; Tambini, Ketz, \& Davachi, 2010).

The temporal window for visual short-term consolidation may last about 500ms into the retention interval (Chun \& Potter, 1995; Giesbrecht \& Di Lollo, 1998; Jolicoeur \& Dell'Acqua, 1998; Lalonde \& Chaudhuri, 2002; Shapiro, Raymond, \& Arnell, 1994; Vogel, Woodman, \& Luck, 2006). Earlier studies using the attentional blink paradigm showed that identification of a target stimulus is impaired if it is presented $200-500 \mathrm{~ms}$ after an initial stimulus (Chun \& Potter, 1995; Shapiro, et al., 1994). More recent behavioral experiments showed impaired memory performance if a distractor or 'memory mask' is presented up to 500ms after presentation of the memory item (Jolicoeur \& Dell'Acqua, 1998; Magnussen, Greenlee, \& Thomas, 1996; Vogel, et al., 2006). The distractor may interfere with the memory representation of the target stimulus, thereby assessing the window of consolidation more directly (Enns \& Di Lollo, 2000; Magnussen \& Greenlee, 1999).

It remains unclear how sensory cortex contributes to this consolidation window. Here, we investigated the functional role of human early visual areas in VSTM consolidation using transcranial magnetic stimulation (TMS). TMS can be used to manipulate activity in retinotopic visual cortex (e.g. Amassian, et al., 1989; Kammer, Puls, Erb, \& Grodd, 2005; Kammer, Puls, Strasburger, Hill, \& Wichmann, 2005; Kastner, Demmer, \& Ziemann, 1998; Sack, van der Mark, Schuhmann, Schwarzbach, \& Goebel, 2009; Thielscher, Reichenbach, Ugurbil, \& Uludag, 2010) and chart the time course of behavioral relevance of the stimulated area (Pascual-Leone, Walsh, \& Rothwell, 2000). In our study, participants saw a visual stimulus array in either their left or right visual field, and 
had to report if a subsequent test array differed from the previously presented memory array. We administered TMS to one visual field (spatial specificity) at different time points (temporal specificity) during the consolidation window, which allowed us to directly test the functional relevance, topography and temporal characteristics of early visual cortex (EVC) contribution to VSTM.

\section{MATERIALS AND METHODS}

\section{Participants}

We ran a behavioral pilot experiment, for which we recruited eleven participants. Nine out of the eleven participants reached the performance threshold during training (five females; one left-handed; mean (SD) age of 24.8 (2.2) years).

For the TMS experiment we recruited nineteen participants. For fourteen participants, we positioned the TMS coil over the scalp using phosphene localization, of which six participants were excluded (two because of failing to reach the performance threshold during training and four because phosphenes could not be elicited reliably). For five others, we used fMRI-based neuronavigation to position the coil, of which one participant was excluded because of seeing phosphenes during the main part of the experiment. The remaining twelve participants (six females; one left-handed) had a mean (SD) age of 25.3 (3.8) years. The TMS protocol followed published safety guidelines (Rossi, Hallett, Rossini, \& Pascual-Leone, 2009; Wassermann, 1998), and was approved by the Medical Ethics Committee of the University Hospital of Maastricht.

For the memory masking experiment we recruited twelve participants. All participants reached the required performance threshold during training (seven females; four left-handed; mean (SD) age of 20.8 (1.3) years). Participants were recruited from the academic environment of Maastricht University, The Netherlands. All participants provided written informed consent prior to participation, and TMS participants also filled out and signed health screening forms that were approved by a medical supervisor. All participants received financial compensation or course credits for their participation. 


\section{Experimental design}

\section{Stimulus material and presentation}

Pictures were grey surfaces (luminance, $68.7 \mathrm{~cd} / \mathrm{m}^{2}$ ) of non-natural shapes (BORTS: blurred outlines of random Tetris shapes; courtesy of N. Kriegeskorte), which could not be easily verbalized or conceptualized (Linden, et al., 2003; Peters, Goebel, \& Roelfsema, 2009). Pictures spanned $1.5^{\circ}$ in width and height, and were drawn on a white background $\left(227 \mathrm{~cd} / \mathrm{m}^{2}\right)$ that spanned the entire computer screen $\left(34^{\circ} \times 27^{\circ}\right.$ visual angle). For the pilot and memory masking experiments (see below), we created a distractor stimulus (memory mask) from the average of all the abstract shapes used in the experiments. Participants were seated in front of a PC-controlled computer screen (refresh rate, $60 \mathrm{~Hz}$ ) at an eye-screen distance of $\sim 57 \mathrm{~cm}$. Stimulus presentation and timing, and logging of individual responses were controlled using the Presentation software (Neurobehavioral Systems Inc.).

The experimental task was an adapted version of the change detection task. A memory trial (Figure 1) started with a brief presentation $(500 \mathrm{~ms})$ of an arrow at the fixation location to indicate in which visual field the trial would be presented. The arrow was briefly replaced by a fixation symbol ++ sign, duration $500 \mathrm{~ms}$ ), after which the remainder of the trial started, including i) presentation of the sample stimulus array, ii) retention of the sample in working memory, iii) presentation of the test stimulus array and iv) the participant's response.

The sample array contained one or three grey-scale abstract pictures, which were presented simultaneously. Pictures were centered at an eccentricity of $6^{\circ}$ visual angle in the lower visual fields on either the left or right side of the fixation symbol. The respective polar angle of the pictures were $25^{\circ}, 45^{\circ}$ and $65^{\circ}$ from the horizontal meridian in the left and right lower quadrants (Figure 1.A shows stimulus presentation for the lower left visual field, LVF). The sample array was presented for $150 \mathrm{~ms}$, and was followed by a blank screen and superimposed fixation symbol for $1500 \mathrm{~ms}$, during which the sample array had to be retained in memory. A test array followed the retention period, at the same location as the sample array. The test array contained either the same pictures as the sample array or was different from the sample array in one picture. For the stimulus arrays of three pictures the altered picture had an equal probability to be presented at any of the three positions. Participants were required to judge if the test array was the same as the sample array (i.e., SAME response, pressing the ' $\mathrm{Z}$ ' key with the left hand) or not (DIFFERENT response, pressing the ' $/$ ' key with the right hand). Participants were instructed that accuracy was more important than response speed, but that there was a time limit for response of 5 seconds. 

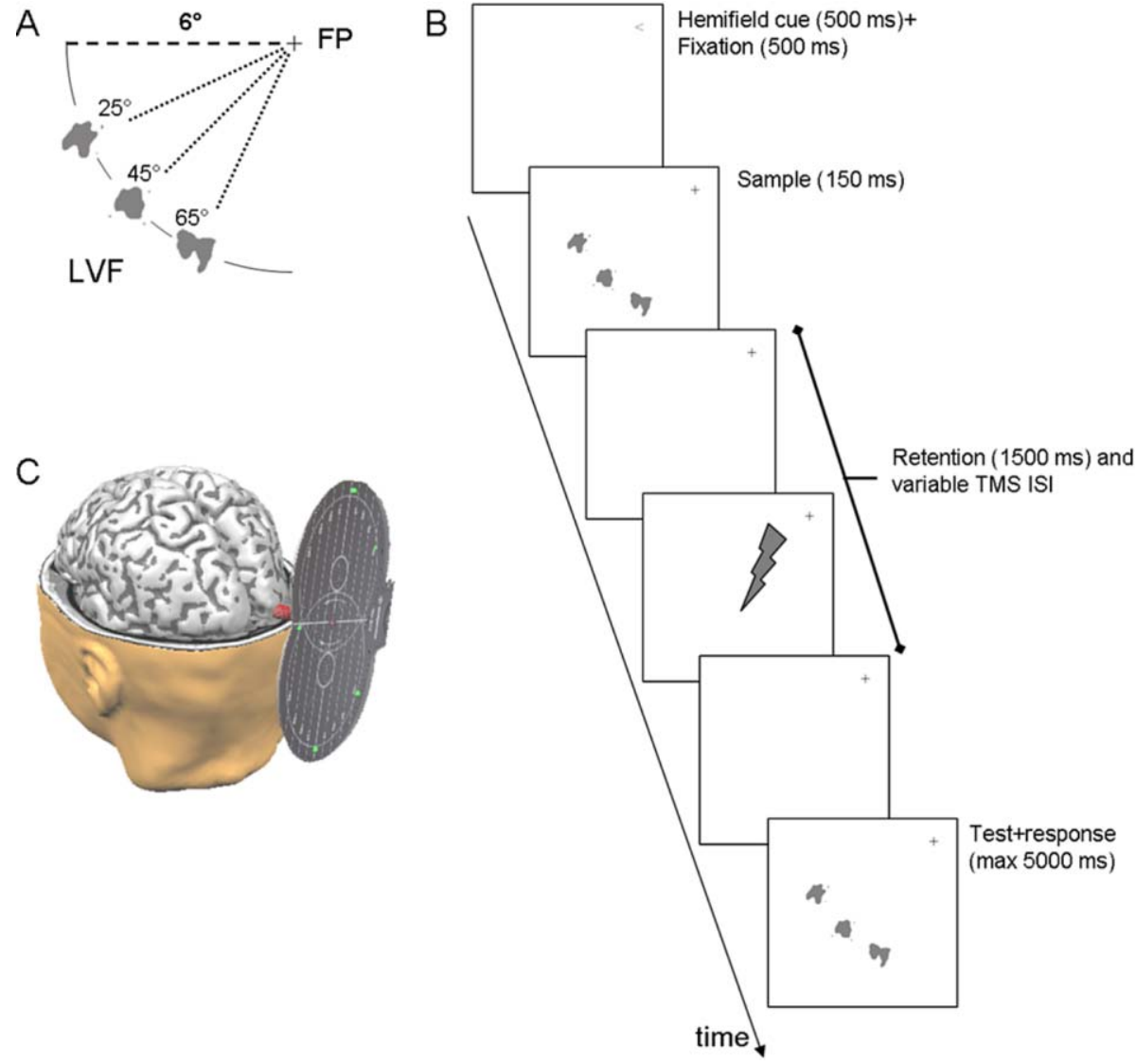

Figure 1. Experimental design. The memory task was a modified version of the change detection task that contained a sample presentation phase, a retention phase and a test phase. A) Sample and test stimulus arrays contained sample and test arrays of one (low memory load) or three (high load) simultaneously presented abstract shapes, which were always presented at either the left or the right side of the fixation point (FP), at $6^{\circ}$ visual angle, and dispersed at equal distances of $20^{\circ}$ polar angle steps. B) A memory trial started with a short presentation of a visual field cue (' $<$ ' or ' $>$ ') that designated the visual field in which the trial would be presented (cue validity $=100 \%$ ). Afterwards, the sample array was briefly shown, and was followed by a retention interval of fixed duration $(1500 \mathrm{~ms})$. Finally, the retention interval was followed by presentation of the test array and participants were required to judge if the test array was the same as the sample array (i.e., match) or not (non-match). For TMS trials, a single TMS pulse was delivered at 100, 200 or $400 \mathrm{~ms}$ into the retention interval (inter-stimulus-interval [ISI]). For baseline measurements, no pulses were delivered. C) TMS pulses were delivered over lateral occipital cortex, using the ipsilateral visual field as TMS-naive control. 


\section{General procedure}

Participants first completed a number of practice sessions, in which twentyfour change detection trials were presented (4 blocks of 6 trials, 2 blocks in each visual field). Participants were required to attain a minimum accuracy of $20 / 24$ trials, which was commonly attained within two to five sessions. For each practice trial, participants received feedback about their performance (green [red] fixation cross indicated correct [false] judgment). Afterwards, participants completed a baseline session (60 trials across 2 hemifields and 2 memory load conditions; 6 blocks of 10 trials each) during which no TMS pulses or masking stimuli were delivered. Finally, participants completed the main memory experiment, in which a TMS pulse or memory mask was presented at one of three time points during the retention interval (i.e., after offset of the sample array; inter-stimulus-interval [ISI] of 100, 200 and $400 \mathrm{~ms}$; 60 trials per time point; 18 blocks of 10 trials each). Consecutive trials in each block were separated by $3000 \mathrm{~ms}$ during which participants only saw the fixation cross at the center of the screen. Visual field position of the memory trials varied in a block-by-block fashion (i.e., left-right-left). During the training and baseline experiments Memory load was randomized within and across blocks. During the TMS and memory masking experiments Memory load and Time point of interference (TMS pulse or memory mask) were randomized within and across blocks, with equal probability for each Load $\mathrm{x}$ Time point combination. In the pilot experiment, the location of the memory mask was always spatially congruent with the memory trial. In the TMS and the memory masking experiment, the pulse or memory mask was presented at a fixed visual field location, so that it was spatially congruent to $50 \%$ of the memory trials. Specifically, in the TMS experiment, pulses were delivered to the left (right) visual field in eleven (one) participants. In the memory masking experiment, distractor stimuli were presented in the left (right) visual field in eight (four) participants.

\section{Transcranial magnetic stimulation}

Biphasic TMS pulses were delivered over lateral visual cortex using a figure-ofeight coil (MC-B70, the inner and outer radii of the two coil loops are 1.2 and $5.4 \mathrm{~cm}$, respectively) (Figure 1.C) and a Medtronic MagPro R30 stimulator (Medtronic Functional Diagnostics A/S, Skovlunde, Denmark; maximum stimulator output, 1.9T).

We used phosphene localization to determine the coil position over the scalp in eight participants (Campana, Cowey, \& Walsh, 2002; Cattaneo, Vecchi, Pascual-Leone, \& Silvanto, 2009; Pascual-Leone \& Walsh, 2001; Romei, et al., 2008; Sack, van der Mark, et al., 2009; Silvanto, Cowey, Lavie, \& Walsh, 2005). Here, the location and intensity of pulse delivery was determined by localizing TMS-induced phosphenes as close as possible to the visual field location of the 
center stimulus of the sample array. TMS-induced phosphenes have been associated with local transient changes in activity of early visual areas (Amassian, et al., 1989; Kammer, Puls, Erb, et al., 2005; Meyer, Diehl, Steinmetz, Britton, \& Benecke, 1991).

Phosphene localization was performed with a black computer screen, which facilitated phosphene detection and localization. Phosphene localization started with $60 \%$ of maximum stimulator output intensity, the center of the coil was positioned about $2 \mathrm{~cm}$ above the inion. The coil was then moved laterally and single TMS pulses were applied until a clear phosphene was perceived in the contralateral visual field. Phosphene perception had to depend on stimulated hemisphere and retinotopic organization in order to be considered a valid TMSinduced percept (Meyer, et al., 1991; Sack, van der Mark, et al., 2009). This procedure aimed at positioning the TMS coil in such a way that the induced phosphenes overlapped with or were in close proximity to the spatial location of the sample and test arrays. We used custom-written software to allow participants to monitor and register phosphene locations in the visual field. After localization, the TMS coil was fixed in a mechanical arm coil holder and placed tangentially on the skull. Three phosphenes were then elicited to ensure that phosphene location was not altered. In seven of the eight participants we administered TMS pulses to the right hemisphere (left visual field), thereby using the left hemisphere as control. In one participant, reliable phosphenes could only be elicited in the left hemisphere (right visual field), with the right hemisphere used as control.

Phosphene localization is inherently based on subjective reports of phosphene perceptions, in which phosphenes may not be reliably produced in some participants. Therefore, we used fMRI-based neuronavigation (Sack, Cohen Kadosh, et al., 2009) to position the coil over the V1/V2 complex of the upper convex of the calcarine fissure (lower visual field) of the right hemisphere in four other participants. Neuronavigation was based on the results of an fMRI localizer of the stimulus position in the lower left visual field ( $6^{\circ}$ eccentricity), which was acquired in a separate, unrelated study (De Weerd, et al., 2012). Note that for these participants the coil position was independent of whether, or how reliable phosphenes could be induced. TMS neuronavigation followed procedures described elsewhere (Sack, Cohen Kadosh, et al., 2009). After coil positioning we attempted to induce phosphenes. Two participants saw phosphenes in the same part of the visual field as did the participants in whom coil position was based on phosphene localization. We could not elicit phosphenes in the other two participants.

During the main experiment single-pulse TMS was applied at stimulator output intensity equal to $110 \%$ of phosphene threshold to correct for the white background of the screen during presentation of the memory experiment. Visual exposure to dimmed or dark luminance markedly decreases phosphene thresholds (Boroojerdi, et al., 2000). Stimulus presentation and pulse triggering were controlled using Presentation (Neurobehavioral Systems, Inc., Albany, 
CA). For the two participants who did not see phosphenes, stimulation intensity during the remainder of the experiment was set at 45\% machine output.

\section{Analysis}

The dependent variable in all experiments was accuracy, which was estimated according to signal detection theory (SDT) rationale that a decision criterion to correctly judge the presence of a signal results from the deviation between signal and noise distributions (MacMillan \& Creelman, 2005). Hit rate (H) was calculated as the number of hits (correctly judging that sample and probe were the same) divided by the sum of number of hits and misses (i.e., $\mathrm{H}=$ hits / (hits + misses)). False alarm rate (FA) was calculated as the number of false alarms (erroneously judging that sample and probe were the same) divided by the sum of the number of false alarms and correct rejections (i.e., FA $=$ false alarms / (false alarms + correct rejections)). We used the non-parametric A' (A-prime) index (MacMillan \& Creelman, 2005; Snodgrass \& Corwin, 1988; Stanislaw \& Todorov, 1999) as a measure of accuracy independent of response bias. Originally, $A^{\prime}$ is estimated using two formulas:

$$
\begin{aligned}
& A^{\prime}=.5+\frac{(H-F A)(1+H-F A)}{4 H(1-F A)}, \text { when } \mathrm{H}>\mathrm{FA} \text {, and } \\
& A^{\prime}=.5-\frac{(F A-H)(1-F A+H)}{4 F A(1-H)}, \text { when } \mathrm{FA}>\mathrm{H} .
\end{aligned}
$$

These equations can be rewritten into a single formula, as follows (Stanislaw \& Todorov, 1999):

$$
A^{\prime}=.5+\left[\operatorname{sign}(H-F A) \frac{(H-F A)^{2}+|H-F A|}{4 \max (H, F A)-4 H F A}\right]
$$

$A^{\prime}$ presents a non-parametric alternative to the more often used d' (d-prime) when assumptions of normality that are required for d' are suspect to violation (MacMillan \& Creelman, 2005; Stanislaw \& Todorov, 1999). In our case, we used A' rather than d' because we obtained a relatively small sample of responses per cell, and because some participants showed perfect performance (i.e. H $=1$ ) in some conditions. We also ran the main analyses using d', and results were similar.

Accuracy was analyzed using repeated-measures analysis-of-variance (RMANOVA) models. For the pilot experiment, we first verified that responses in the two visual fields were not significantly different, using a Field (left, right) $\mathrm{x}$ 
Load $(1,3)$ x ISI $(100,200,400)$ RM-ANOVA model with first and second order interaction terms. We then proceeded with a $\operatorname{Load}(1,3)$ x Mask $(100,200,400)$ RM-ANOVA model that also included the interaction term. For the TMS experiment, we used a Field (TMS, Control) x Load $(1,3) \times$ TMS $(100,200,400)$ RM-ANOVA model with first and second order interaction terms. We used a similar model for analysis of the memory masking experiment [i.e., Field (Mask, Control) x Load $(1,3) \times$ ISI $(100,200,400)$, with first and second order interaction terms]. Effects with p-values at or below the alpha level of 0.05 were considered significant. Post hoc comparisons of mean differences were performed using two-tailed one-sample t-tests (Holm-Bonferroni corrected for multiple comparisons). Effect sizes for RM-ANOVA were further estimated for significant effects (partial eta squared, $\eta^{2}$, for ANOVA effects and Cohen's $d$ for t-test effects).

\section{RESULTS}

\section{Pilot experiment of short-term memory and visual masking}

In this experiment, we presented lateralized short-term memory trials and memory masks that were always spatially congruent with the memory trials, in order to investigate the effect of memory masking independent of visual field. In an initial analysis we verified that memory masks presented in each visual field provided similar response patterns. We analyzed percentage accuracy of memory performance using a 2 × 2 × 3 RM-ANOVA with factors Visual Field (left or right), Load (1 or 3) and ISI $(100,200$ or 400$)$. We found a significant main effect of Load $[F(1,8)=238.2, p<0.001]$, but no significant effects related to Field $[\mathrm{F}(1,8)=1.02, \mathrm{p}=0.34]$, which confirmed that memory masking did not differentially affect performance of the two visual fields. Further, we found trend effects for ISI $[\mathrm{F}(1,8)=3.0, \mathrm{p}=0.079]$ and for the Load $\mathrm{x} I S I$ interaction $[\mathrm{F}(2,16)=3.1, \mathrm{p}=0.073]$.

To obtain more power, we pooled the results across the two visual fields, and recalculated a $2 \times 3$ RM-ANOVA (Load x ISI). We found a significant main effect of Load $[\mathrm{F}(1,8)=241.0, \mathrm{p}<0.001, \eta 2=0.97]$, and a significant Load $\mathrm{x}$ ISI interaction effect $[F(2,16)=3.6, p=0.052, \eta 2=0.31]$. Figure 2 shows the mean accuracies across all conditions (data pooled across Field; see also Table 1). The interaction effect was based on a decrease of accuracy when the mask was presented $200 \mathrm{~ms}$ into the retention interval [mean $\mathrm{A}^{\prime}=0.71$ ], compared to the other two time points [mean A' ISI $100=0.78$; ISI $400=0.78$ ], only during high memory load trials (see Table 1). These results indicated that presentation of a memory mask around $200 \mathrm{~ms}$ into the retention interval interfered with subsequent recognition in high memory load trials. 

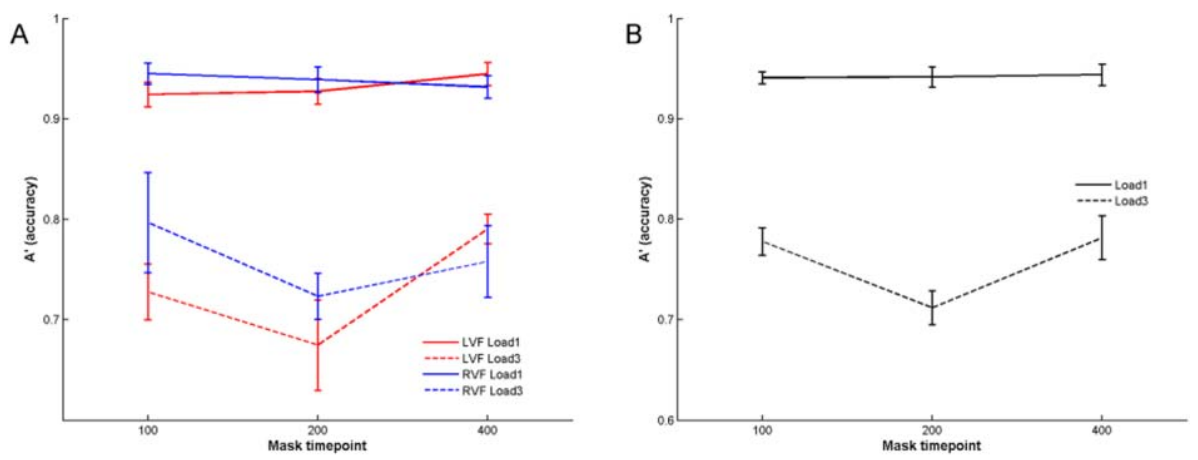

Figure 2. Pilot results. Plots show accuracy (A') of memory trials with memory masks at 100, 200 or $400 \mathrm{~ms}$ into the retention interval. Here, memory masks were always spatially congruent to the memory trial. A) Mean accuracies are shown for the two memory loads (load 1, solid lines; load 3, broken lines) and for the two visual fields (left visual field [LVF], red; right visual field [RVF], blue). Analysis-of-variance showed that responses were similar across the two visual fields. B) Mean accuracies are shown for the two memory loads collapsed across visual fields. Error bars represent one standard error of the mean (1 SEM).

\section{Short-term memory and TMS}

In this experiment, we presented lateralized short-term memory trials while TMS pulses were always delivered over retinotopic occipital cortex (affecting one visual field location). Thus, TMS pulses were spatially congruent with $50 \%$ of the memory trials.

Accuracy was analyzed using a $2 \times 2 \times 3$ RM-ANOVA model (Field $\mathrm{x}$ Load $\mathrm{x}$ TMS). To verify that the results were not biased by the two different methods of coil positioning, we added Positioning Method (phosphenes, neuronavigation) as between-subject factor.

With respect to Positioning Method, we found a significant Load x Method interaction effect $[F(1,10)=5.2, p=0.046, \eta 2=0.34]$. Participants of the neuronavigation method showed slightly better performance for low memory load trials [mean (SD) $\left.A^{\prime}=0.94(0.04)\right]$ compared to those of phosphene localization [mean (SD) $\left.A^{\prime}=0.89(0.04)\right]$, but slightly worse performance for high memory load trials [mean (SD) $\left.A^{\prime}=0.64(0.11)\right]$ than those of phosphene localization [mean (SD) $\left.A^{\prime}=0.71(0.12)\right]$. All other interactions with Method were not significant (Ps > 0.22). Thus, localization method did not affect the pattern of results. We pooled the data of participants from both positioning methods in the following analyses. 


\begin{tabular}{|c|c|c|c|c|c|c|}
\hline \multirow[b]{2}{*}{ Pilot } & \multicolumn{3}{|c|}{ Load 1} & \multicolumn{3}{|c|}{ Load 3} \\
\hline & 100 & 200 & 400 & 100 & 200 & 400 \\
\hline $\begin{array}{l}\text { Mask } \\
{[\mathrm{L}+\mathrm{R}]}\end{array}$ & $\begin{array}{l}0.94 \\
{[0.03]}\end{array}$ & $\begin{array}{l}0.94 \\
{[0.03]}\end{array}$ & $\begin{array}{l}0.94 \\
{[0.02]}\end{array}$ & $\begin{array}{l}0.78 \\
{[0.05]}\end{array}$ & $\begin{array}{l}0.71 \\
{[0.06]}\end{array}$ & $0.78[0.08]$ \\
\hline TMS & $\begin{array}{l}0.89 \\
{[0.09]}\end{array}$ & $\begin{array}{l}0.90 \\
{[0.13]}\end{array}$ & $\begin{array}{l}0.93 \\
{[0.05]}\end{array}$ & $\begin{array}{l}0.70 \\
{[0.20]}\end{array}$ & $\begin{array}{l}0.54 \\
{[0.26]}\end{array}$ & $0.74[0.18]$ \\
\hline Control & $\begin{array}{l}0.92 \\
{[0.07]}\end{array}$ & $\begin{array}{l}0.90 \\
{[0.11]}\end{array}$ & $\begin{array}{l}0.91 \\
{[0.06]}\end{array}$ & $\begin{array}{l}0.75 \\
{[0.22]}\end{array}$ & $\begin{array}{l}0.76 \\
{[0.17]}\end{array}$ & $0.63[0.21]$ \\
\hline Mask & $\begin{array}{l}0.95 \\
{[0.11]}\end{array}$ & $\begin{array}{l}0.94 \\
{[0.13]}\end{array}$ & $\begin{array}{l}0.95 \\
{[0.09]}\end{array}$ & $\begin{array}{l}0.75 \\
{[0.13]}\end{array}$ & $\begin{array}{l}0.68 \\
{[0.10]}\end{array}$ & $0.77[0.14]$ \\
\hline Control & $\begin{array}{l}0.94 \\
{[0.05]}\end{array}$ & $\begin{array}{l}0.96 \\
{[0.13]}\end{array}$ & $\begin{array}{l}0.94 \\
{[0.14]}\end{array}$ & $\begin{array}{l}0.79 \\
{[0.16]}\end{array}$ & $\begin{array}{l}0.79 \\
{[0.14]}\end{array}$ & $0.74[0.14]$ \\
\hline
\end{tabular}

Table 1. Memory performance. Mean [SD] accuracies (A') of the three experiments according to memory load (one or three items) and TMS or masking time point (100, 200 or $400 \mathrm{~ms}$ into the retention interval). For the pilot study, performance in the left (L) and right (R) visual fields are pooled because memory masks were always spatially congruent with the memory trials.

For the within-subject factors, we found a significant main effect of Load $[\mathrm{F}(1,11)=57.8, \mathrm{p}<0.001, \eta 2=0.84]$, a significant Field $\mathrm{x}$ TMS interaction effect $[\mathrm{F}(2,22)=3.9, \mathrm{p}=0.037, \eta 2=0.26]$ and a significant Field $\mathrm{x}$ Load $\mathrm{x}$ TMS interaction effect $[F(2,22)=4.3, p=0.026, \eta 2=0.28]$. To parcel out these effects, we calculated $2 \times 3$ RM-ANOVAs for each of the two memory loads. For Load 1 we found no significant main or interaction effects (Ps $>0.59$ ). For Load 3 we found a significant Field $x$ TMS interaction effect $[\mathrm{F}(2,22)=5.0, \mathrm{p}=0.016, \eta 2=0.31]$. Figure 3 shows the mean accuracies across the conditions (see also Table 1).

These results corroborated and extended the pilot memory masking results in the following way. The interactions were based on a decrease of accuracy when the spatially congruent TMS pulse was presented $200 \mathrm{~ms}$ into the retention interval, compared to the spatially incongruent pulse, only for the high memory load (see Table 1). Post hoc contrasts between TMS and control visual fields for the time points during high memory load trials showed a significant TMS-induced impairment in performance at the $200 \mathrm{~ms}$ time point [mean decrease $A^{\prime}=-0.21 ; t(11)=-2.9, p=0.013$, Cohen's $\left.d=0.85\right]$, but not at the other two time points. Further post hoc comparisons in the TMS condition of the decrease in accuracy at $200 \mathrm{~ms}$ compared to the other two time points were significant $\left(\left[\mathrm{A}^{\prime}{ }_{100}>\mathrm{A}^{\prime}{ }_{200}\right]: \mathrm{t}(11)=-1.9, \mathrm{p}=0.044 ;\left[\mathrm{A}_{400}^{\prime}>\mathrm{A}_{200}^{\prime}\right]: \mathrm{t}(11)=-2.3, \mathrm{p}=\right.$ $0.021)$. We found no significant differences within the control condition. 

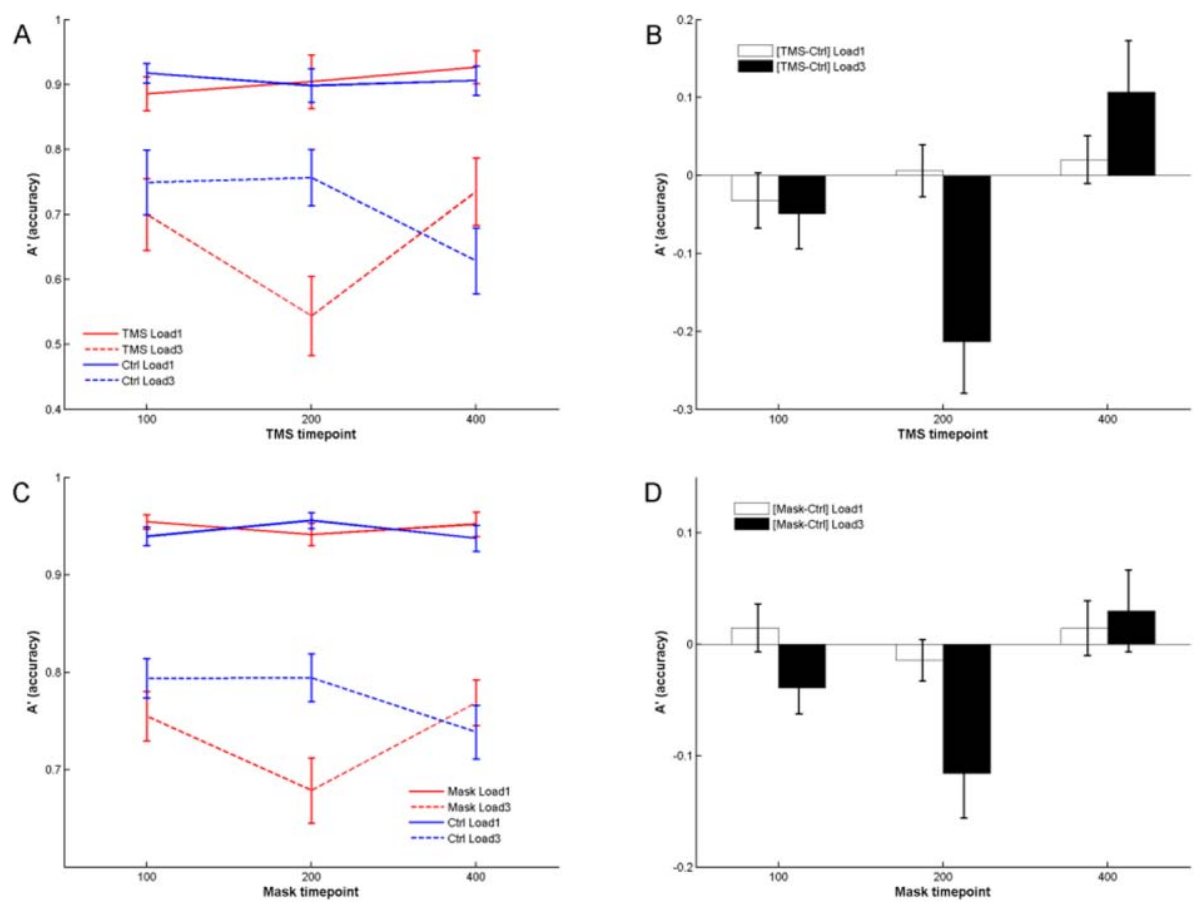

Figure 3. TMS and memory masking results. Plots show accuracy (A') of memory trials with TMS pulses (A,B) or memory masks (C,D) at 100, 200 or $400 \mathrm{~ms}$ into the retention interval. Here, TMS pulses and memory masks were spatially congruent to the memory trial in $50 \%$ of the trials. A, C) Mean accuracies are shown for the two memory loads and two visual fields after presentation of a TMS pulse (A) or memory mask (C). B, D) Mean interference effect of accuracy after presentation of a TMS pulse (B: TMS visual field - Control visual field) or a memory mask (D: Mask visual field Control visual field). Error bars represent 1 SEM. TMS/Mask, TMS or memory mask visual field; Ctrl, control visual field.

\section{Short-term memory and visual masking}

In this experiment, we presented lateralized short-term memory trials while memory masks were always presented in one visual field location. Thus, memory masks were spatially congruent with $50 \%$ of the trials. Accuracy was analyzed using a $2 \times 2 \times 3$ RM-ANOVA model (Field x Load x ISI). We verified that the choice of control visual field (right in eight participants, left in four) did not affect the results by adding Mask Location (left, right) as between-subject factor. Mask Location did not show interaction effects with any of the within-subject factors (Ps > 0.32), which further corroborated the finding from the pilot study that memory performance did not differ between visual fields.

For the within-subject factors, we found a significant main effect of Load $[\mathrm{F}(1,11)=239.1, \mathrm{p}<0.001, \eta 2=0.96]$ and a significant Field $\mathrm{x}$ ISI interaction effect $[F(2,22)=5.5, p=0.012, \eta 2=33]$. To parcel out these effects, we recalcu- 
lated $2 \times 3$ RM-ANOVAs for each of the two memory loads (i.e., Field $\times$ ISI). For Load 1 we found no significant main or interaction effects (Ps > 0.31). For Load 3 we found a significant Field $x$ TMS interaction effect $[\mathrm{F}(2,22)=4.1, \mathrm{p}=0.031$, $\eta 2=0.27$ ]. Figure 3 shows the mean accuracies across the conditions (see also Table 1).

These results corroborated and extended the pilot and TMS results in the following way. The interactions were based on a decrease of accuracy when the spatially congruent mask was presented $200 \mathrm{~ms}$ into the retention interval, compared to the spatially incongruent mask (see Table 1). This effect was present only for the high memory load. Post hoc contrasts between mask and control visual fields for the $200 \mathrm{~ms}$ time point showed that the mask-induced performance impairment was marginally significant at the uncorrected P-value [mean decrease $\mathrm{A}^{\prime}=-0.12 ; \mathrm{t}(11)=-2.1, \mathrm{p}=0.056$, Cohen's $\mathrm{d}=0.62$ ], but not at the corrected P-value. The other two masking time points showed no significant differences (Ps > 0.7). Further post hoc comparisons in the TMS condition of the decrease in accuracy at $200 \mathrm{~ms}$ compared to the other two time points were significant $\left(\left[\mathrm{A}^{\prime}{ }_{100}>\mathrm{A}^{\prime}{ }_{200}\right]: \mathrm{t}(11)=-2.1, \mathrm{p}=0.028 ;\left[\mathrm{A}^{\prime}{ }_{400}>\mathrm{A}^{\prime}{ }_{200}\right]: \mathrm{t}(11)=-2.4, \mathrm{p}=\right.$ $0.016)$. We found no significant differences within the control condition. These results suggest that participants showed a similar pattern of interference at 200 $\mathrm{ms}$, compared to the TMS experiment.

\section{DISCUSSION}

To investigate the functional relevance, topography and temporal characteristics of early visual cortex (EVC) contribution to VSTM consolidation, we presented memory masks or administered topographically-specific TMS pulses affecting the visual field contralateral to the side of TMS at different time points within $100-400 \mathrm{~ms}$ into the retention interval of a change detection task with low and high memory loads, and used the other visual field as within-subject control. We found a very strong correspondence in memory interference between the TMS and masking experiments. Specifically, memory masks and TMS pulses decreased accuracy only in the targeted visual field, revealing the topographic specificity of our TMS-induced memory consolidation interference in EVC. Furthermore, accuracy in the targeted visual field decreased only when the memory mask or TMS pulse was administered at $200 \mathrm{~ms}$ into the retention window (temporal specificity), and most strongly for the high memory load (capacity limit). These findings not only provide direct evidence for the functional necessity of intact neural processing in EVC at 200ms into the retention phase, but also suggest a load dependence of this functional relevance in memory consolidation. The similar findings of both experiments suggest that interference of activity in visual cortex reliably impaired memory consolidation, thereby affecting memory performance. Our findings are in line with previous interference studies that showed a critical window for short-term consolidation up to 500ms after stimulus offset (Chun \& Potter, 1995; Giesbrecht \& 
Di Lollo, 1998; Jolicoeur \& Dell'Acqua, 1998; Lalonde \& Chaudhuri, 2002; Magnussen, et al., 1996; Vogel, et al., 2006), and reveal a neurophysiological correlate of this consolidation window that includes activation states of topographic EVC, which suggests that VSTM representations may be formed or maintained in sensory brain areas that encoded the stimuli. To our knowledge, we are the first to show that topographic EVC is functionally relevant for shortterm consolidation of sensory visual information.

Up to date, very few studies have investigated the role of occipital cortex in VSTM. In an early study (Beckers \& Homberg, 1991), participants completed a delayed match-to-sample (DMS) task of face stimuli, during which TMS was applied over the occipital pole before (i.e., during the retention phase) or after presentation of the memory probe (i.e., during memory scanning). The authors found no significant change in reaction times after pulses were delivered during the memory retention phase. However, pulses delivered during the memory scanning phase significantly decreased the scanning rate. A more recent TMS study found that TMS administered at the onset of the DMS retention phase increased reaction times, whereas TMS at the end of the retention phase decreased reaction times (Cattaneo, et al., 2009). Crucially, in these studies effects of TMS on memory performance were found in altered reaction times, but not in accuracy, which suggests that their effects may be mediated by other neural mechanisms as compared to our study.

TMS may interfere with signal processing by decreasing signal strength, rather than adding random noise (Harris, Clifford, \& Miniussi, 2008). Following, TMS may decrease the strength of, or overwrite the neural representation of the memory trace in visual cortex. This suggestion is in line with propositions of object substitution in visual memory after presentation of a competing stimulus (Enns \& Di Lollo, 2000; Giesbrecht \& Di Lollo, 1998). Furthermore, our finding of a lateralized TMS effect on memory performance suggested that the neural memory representation was retinotopically organized, with limited transfer of memorized information to other parts of the visual field (Dill \& Fahle, 1997; Karni \& Sagi, 1993; Schoups, Vogels, \& Orban, 1995). This spatial specificity of our findings further supports the notion of a local neural representation of memory in visual cortex. Importantly, the interference of TMS on memory performance was not due to impaired visual awareness of the to-be memorized items. Visual awareness is commonly impaired if a visual mask is presented at $60-120 \mathrm{~ms}$ after a very brief presentation of the target stimulus (Breitmeyer \& Ogmen, 2000). A number of TMS studies that presented pulses in a similar temporal window reported similar impairments of awareness (Amassian, et al., 1989; Beckers \& Homberg, 1991; Kammer, 2007; Sack, van der Mark, et al., 2009). In our study, participants were not impaired in their memory performance if TMS or the mask was presented at $100 \mathrm{~ms}$ into the retention interval. Instead, TMS impaired memory performance at a later time window, and only for the high memory load, which suggests that TMS pulses interfered with post-stimulus processes beyond those of initial encoding. 
Also, it is unlikely that TMS decreased accuracy through phosphenes acting as visual masks. Most participants reported seeing no or few phosphenes during the memory experiment (see also Cattaneo, et al., 2009; Silvanto, et al., 2005). Further, even if participants saw phosphenes but failed to report them, 'phosphene masking' cannot explain the larger TMS-induced impairment, compared to the smaller impairment in the memory masking experiment. Finally, TMSinduced phosphenes could perhaps increase memory performance. Silvanto and colleagues showed that TMS-induced phosphenes may contain previously learned or memorized information (Silvanto \& Cattaneo, 2010; Silvanto, Muggleton, Cowey, \& Walsh, 2007). Participants could possibly use such phosphenes to rehearse the information during retention and increase recognition accuracy.

Alternatively, TMS may have interfered with ongoing communication between visual cortex and higher-level areas that may occur during periods of consolidation, as is shown in recent fMRI studies (Lewis, Baldassarre, Committeri, Romani, \& Corbetta, 2009; Tambini, et al., 2010). Furthermore, the time point of impairment may be associated with dynamically altering states of brain activity in sensory and higher-level areas. For example, Lamme and Roelfsema (2000) proposed that visual perception is the result of sweeps of activity running up and down the visual hierarchy. Incoming visual sensory information is initially processed in a feedforward sweep up the hierarchy that occurs within 40 to $70 \mathrm{~ms}$ after stimulus onset. Higher level brain areas then feedback information to early visual areas within 80 to $120 \mathrm{~ms}$ after stimulus onset. The feedforward sweep may be associated with initial stimulus encoding, while the feedback sweep may be associated with visual awareness or attention. In our study, the time point of interference may indicate that alteration of activity in EVC may have affected a later feedback sweep to EVC during short-term memory consolidation, suggesting that memory consolidation requires additional forward and backward sweeps beyond those needed for visual awareness.

Finally, we found that the functional relevance of EVC for memory consolidation depended on the capacity constraints of VSTM (Cowan, 2000; Luck \& Vogel, 1997). Retaining memory loads that approach the capacity limit is more easily or more profoundly impaired by the processing of distractors (Cowan, 2000; Vogel, et al., 2006). Previous fMRI studies showed a neural correlate for the capacity limitation of VSTM in frontal and parietal cortex (Linden, et al., 2003; Todd \& Marois, 2004), in which brain activity increased monotonically with higher memory loads until the capacity limit was reached. Furthermore, retention of higher memory loads may be associated with increased functional coupling between these brain areas (Honey, et al., 2002; Woodward, et al., 2006). Thus, higher memory loads may require more neural resources for memory retention, which may leave limited resources available to protect against interfering signals. This suggestion may further be relevant when considering that we used complex memory items. Previous studies have shown that increased object complexity may constitute a higher information 
load, which thereby limits the object capacity of short-term memory (Alvarez \& Cavanagh, 2004; Eng, Chen, \& Jiang, 2005; Luria, Sessa, Gotler, Jolicoeur, \& Dell'Acqua, 2010). Following, complexity-imposed shrinkage in VSTM capacity may be particularly costly when the number of memory items approaches the capacity limit. In this sense, trials of high memory and information load should be more prone to interfering signals, which is exactly what we found. However, the nature of the TMS-induced interference on the memory representation in EVC may be different for simple stimuli, such as color or gratings.

The larger TMS-induced impairment compared to that after memory masking may indicate that the nature of the TMS interference may be different from that of memory masking. TMS pulses may have directly affected relevant neural populations, interfering with cortical activity as the memory was processed. In contrast, the memory mask was presented to the retina, and traversed the same neural pathways as did the to-be remembered stimuli. Here, interference effects may be much more subtle. Alternatively, the memory mask may not have provided enough overlap with relevant stimulus dimensions of the abstract shapes, thereby limiting its interference on memory-related processing of the target stimuli (Lalonde \& Chaudhuri, 2002; Magnussen \& Greenlee, 1999).

In conclusion, we showed that EVC topographically contributes to consolidation of visual information in VSTM rather early within the retention window. Furthermore, TMS likely decreased the signal strength of the memory representation, which affects performance most strongly when the memory load approaches the capacity limit of VSTM. Finally, the specific time point of interference appears in line with a sequential pattern of discrete temporal windows during which visual cortex contributes to visual perception.

\section{ACKNOWLEDGMENTS}

The authors kindly thank drs. David E.J. Linden and Christoph Bledowski for very helpful comments on a previous version of the manuscript, and Peter Schubert and Liselot Kerpershoek for assistance in data acquisition. V.V. and A.T.S. were supported by grants from the Netherlands Organization for Scientific Research (NWO; respective grant numbers 451-07-014 and 452-06-003). We thank our medical supervisor Cees van Leeuwen and independent physician Martin van Boxtel. 


\section{REFERENCES}

Alvarez, G. A., \& Cavanagh, P. (2004). The capacity of visual short-term memory is set both by visual information load and by number of objects. Psychol Sci, 15(2), 106-111.

Amassian, V. E., Cracco, R. Q., Maccabee, P. J., Cracco, J. B., Rudell, A., \& Eberle, L. (1989). Suppression of visual perception by magnetic coil stimulation of human occipital cortex. Electroencephalogr Clin Neurophysiol, 74(6), 458-462.

Beckers, G., \& Homberg, V. (1991). Impairment of visual perception and visual short term memory scanning by transcranial magnetic stimulation of occipital cortex. Exp Brain Res, 87(2), 421432.

Boroojerdi, B., Bushara, K. O., Corwell, B., Immisch, I., Battaglia, F., Muellbacher, W., et al. (2000). Enhanced excitability of the human visual cortex induced by short-term light deprivation. Cereb Cortex, 10(5), 529-534.

Breitmeyer, B. G., \& Ogmen, H. (2000). Recent models and findings in visual backward masking: a comparison, review, and update. Percept Psychophys, 62(8), 1572-1595.

Campana, G., Cowey, A., \& Walsh, V. (2002). Priming of motion direction and area V5/MT: a test of perceptual memory. Cereb Cortex, 12(6), 663-669.

Cattaneo, Z., Vecchi, T., Pascual-Leone, A., \& Silvanto, J. (2009). Contrasting early visual cortical activation states causally involved in visual imagery and short-term memory. Eur J Neurosci, 30(7), 1393-1400.

Chun, M. M., \& Potter, M. C. (1995). A two-stage model for multiple target detection in rapid serial visual presentation. J Exp Psychol Hum Percept Perform, 21(1), 109-127.

Courtney, S. M., Ungerleider, L. G., Keil, K., \& Haxby, J. V. (1997). Transient and sustained activity in a distributed neural system for human working memory. Nature, 386(6625), 608-611.

Cowan, N. (2000). The magical number 4 in short-term memory: a reconsideration of mental storage capacity. Behav Brain Sci, 24(1), 87-114; discussion 114-185.

D'Esposito, M., Postle, B. R., \& Rypma, B. (2000). Prefrontal cortical contributions to working memory: evidence from event-related fMRI studies. Exp Brain Res, 133(1), 3-11.

De Weerd, P., Reithler, J., van de Ven, V., Been, M., Jacobs, C., \& Sack, A. T. (2012). Posttraining transcranial magnetic stimulation of striate cortex disrupts consolidation early in visual skill learning. J Neurosci, 32(6), 1981-1988.

Dill, M., \& Fahle, M. (1997). The role of visual field position in pattern-discrimination learning. Proc Biol Sci, 264(1384), 1031-1036.

Eng, H. Y., Chen, D., \& Jiang, Y. (2005). Visual working memory for simple and complex visual stimuli. Psychon Bull Rev, 12(6), 1127-1133.

Enns, J. T., \& Di Lollo, V. (2000). What's new in visual masking? Trends Cogn Sci, 4(9), 345-352.

Fuster, J. M. (1995). Memory in the cerebral cortex: an empirical approach to neural networks in the human and nonhuman primate (First ed.). Cambridge, Massachusetts: MIT Press.

Fuster, J. M., \& Jervey, J. P. (1982). Neuronal firing in the inferotemporal cortex of the monkey in a visual memory task. J Neurosci, 2(3), 361-375.

Giesbrecht, B., \& Di Lollo, V. (1998). Beyond the attentional blink: visual masking by object substitution. J Exp Psychol Hum Percept Perform, 24(5), 1454-1466.

Goldman-Rakic, P. S. (1995). Cellular basis of working memory. Neuron, 14(3), 477-485.

Harris, J. A., Clifford, C. W., \& Miniussi, C. (2008). The functional effect of transcranial magnetic stimulation: signal suppression or neural noise generation? J Cogn Neurosci, 20(4), 734-740.

Harrison, S. A., \& Tong, F. (2009). Decoding reveals the contents of visual working memory in early visual areas. Nature, 458(7238), 632-635.

Honey, G. D., Fu, C. H., Kim, J., Brammer, M. J., Croudace, T. J., Suckling, J., et al. (2002). Effects of verbal working memory load on corticocortical connectivity modeled by path analysis of functional magnetic resonance imaging data. Neuroimage, 17(2), 573-582.

Jolicoeur, P., \& Dell'Acqua, R. (1998). The demonstration of short-term consolidation. Cogn Psychol, 36(2), 138-202.

Jonides, J., Lewis, R. L., Nee, D. E., Lustig, C. A., Berman, M. G., \& Moore, K. S. (2008). The mind and brain of short-term memory. Annu Rev Psychol, 59, 193-224.

Kammer, T. (2007). Masking visual stimuli by transcranial magnetic stimulation. Psychol Res, 71(6), 659-666. 
Kammer, T., Puls, K., Erb, M., \& Grodd, W. (2005). Transcranial magnetic stimulation in the visual system. II. Characterization of induced phosphenes and scotomas. Exp Brain Res, 160(1), 129140.

Kammer, T., Puls, K., Strasburger, H., Hill, N. J., \& Wichmann, F. A. (2005). Transcranial magnetic stimulation in the visual system. I. The psychophysics of visual suppression. Exp Brain Res, 160(1), 118-128.

Karni, A., \& Sagi, D. (1993). The time course of learning a visual skill. Nature, 365(6443), 250-252.

Kastner, S., Demmer, I., \& Ziemann, U. (1998). Transient visual field defects induced by transcranial magnetic stimulation over human occipital pole. Exp Brain Res, 118(1), 19-26.

Lalonde, J., \& Chaudhuri, A. (2002). Task-dependent transfer of perceptual to memory representations during delayed spatial frequency discrimination. Vision Res, 42(14), 17591769.

Lamme, V. A., \& Roelfsema, P. R. (2000). The distinct modes of vision offered by feedforward and recurrent processing. Trends Neurosci, 23(11), 571-579.

Lewis, C. M., Baldassarre, A., Committeri, G., Romani, G. L., \& Corbetta, M. (2009). Learning sculpts the spontaneous activity of the resting human brain. Proc Natl Acad Sci U S A, 106(41), 1755817563.

Linden, D. E., Bittner, R. A., Muckli, L., Waltz, J. A., Kriegeskorte, N., Goebel, R., et al. (2003). Cortical capacity constraints for visual working memory: dissociation of fMRI load effects in a frontoparietal network. Neuroimage, 20(3), 1518-1530.

Luck, S. J., \& Vogel, E. K. (1997). The capacity of visual working memory for features and conjunctions. Nature, 390(6657), 279-281.

Luria, R., Sessa, P., Gotler, A., Jolicoeur, P., \& Dell'Acqua, R. (2010). Visual short-term memory capacity for simple and complex objects. J Cogn Neurosci, 22(3), 496-512.

MacMillan, N., \& Creelman, C. (2005). Detection theory: A user's guide (2nd edition ed.). New Jersey: Psychology Press.

Magnussen, S., \& Greenlee, M. W. (1999). The psychophysics of perceptual memory. Psychol Res, 62(2-3), 81-92.

Magnussen, S., Greenlee, M. W., \& Thomas, J. P. (1996). Parallel processing in visual short-term memory. J Exp Psychol Hum Percept Perform, 22(1), 202-212.

Meyer, B. U., Diehl, R., Steinmetz, H., Britton, T. C., \& Benecke, R. (1991). Magnetic stimuli applied over motor and visual cortex: influence of coil position and field polarity on motor responses, phosphenes, and eye movements. Electroencephalogr Clin Neurophysiol Suppl, 43, 121-134.

Miller, E. K., Erickson, C. A., \& Desimone, R. (1996). Neural mechanisms of visual working memory in prefrontal cortex of the macaque. J Neurosci, 16(16), 5154-5167.

Miyashita, Y., \& Chang, H. S. (1988). Neuronal correlate of pictorial short-term memory in the primate temporal cortex. Nature, 331(6151), 68-70.

Munk, M. H., Linden, D. E., Muckli, L., Lanfermann, H., Zanella, F. E., Singer, W., et al. (2002). Distributed cortical systems in visual short-term memory revealed by event-related functional magnetic resonance imaging. Cereb Cortex, 12(8), 866-876.

Pascual-Leone, A., \& Walsh, V. (2001). Fast backprojections from the motion to the primary visual area necessary for visual awareness. Science, 292(5516), 510-512.

Pascual-Leone, A., Walsh, V., \& Rothwell, J. (2000). Transcranial magnetic stimulation in cognitive neuroscience--virtual lesion, chronometry, and functional connectivity. Curr Opin Neurobiol, 10(2), 232-237.

Pasternak, T., \& Greenlee, M. W. (2005). Working memory in primate sensory systems. Nat Rev Neurosci, 6(2), 97-107.

Peters, J. C., Goebel, R., \& Roelfsema, P. R. (2009). Remembered but unused: the accessory items in working memory that do not guide attention. J Cogn Neurosci, 21(6), 1081-1091.

Romei, V., Brodbeck, V., Michel, C., Amedi, A., Pascual-Leone, A., \& Thut, G. (2008). Spontaneous fluctuations in posterior alpha-band EEG activity reflect variability in excitability of human visual areas. Cereb Cortex, 18(9), 2010-2018.

Rossi, S., Hallett, M., Rossini, P. M., \& Pascual-Leone, A. (2009). Safety, ethical considerations, and application guidelines for the use of transcranial magnetic stimulation in clinical practice and research. Clin Neurophysiol, 120(12), 2008-2039. 
Sack, A. T., Cohen Kadosh, R., Schuhmann, T., Moerel, M., Walsh, V., \& Goebel, R. (2009). Optimizing functional accuracy of TMS in cognitive studies: a comparison of methods. J Cogn Neurosci, 21(2), 207-221.

Sack, A. T., van der Mark, S., Schuhmann, T., Schwarzbach, J., \& Goebel, R. (2009). Symbolic action priming relies on intact neural transmission along the retino-geniculo-striate pathway. Neuroimage, 44(1), 284-293.

Schoups, A. A., Vogels, R., \& Orban, G. A. (1995). Human perceptual learning in identifying the oblique orientation: retinotopy, orientation specificity and monocularity. J Physiol, 483 ( Pt 3), 797-810.

Shapiro, K. L., Raymond, J. E., \& Arnell, K. M. (1994). Attention to visual pattern information produces the attentional blink in rapid serial visual presentation. J Exp Psychol Hum Percept Perform, 20(2), 357-371.

Silvanto, J., \& Cattaneo, Z. (2010). Transcranial magnetic stimulation reveals the content of visual short-term memory in the visual cortex. Neuroimage, 50(4), 1683-1689.

Silvanto, J., Cowey, A., Lavie, N., \& Walsh, V. (2005). Striate cortex (V1) activity gates awareness of motion. Nat Neurosci, 8(2), 143-144.

Silvanto, J., Muggleton, N. G., Cowey, A., \& Walsh, V. (2007). Neural adaptation reveals statedependent effects of transcranial magnetic stimulation. Eur J Neurosci, 25(6), 1874-1881.

Snodgrass, J. G., \& Corwin, J. (1988). Pragmatics of measuring recognition memory: applications to dementia and amnesia. J Exp Psychol Gen, 117(1), 34-50.

Stanislaw, H., \& Todorov, N. (1999). Calculation of signal detection theory measures. Behav Res Methods Instrum Comput, 31(1), 137-149.

Super, H., Spekreijse, H., \& Lamme, V. A. (2001). A neural correlate of working memory in the monkey primary visual cortex. Science, 293(5527), 120-124.

Tambini, A., Ketz, N., \& Davachi, L. (2010). Enhanced brain correlations during rest are related to memory for recent experiences. Neuron, 65(2), 280-290.

Thielscher, A., Reichenbach, A., Ugurbil, K., \& Uludag, K. (2010). The cortical site of visual suppression by transcranial magnetic stimulation. Cereb Cortex, 20(2), 328-338.

Todd, J. J., \& Marois, R. (2004). Capacity limit of visual short-term memory in human posterior parietal cortex. Nature, 428(6984), 751-754.

Vogel, E. K., Woodman, G. F., \& Luck, S. J. (2006). The time course of consolidation in visual working memory. J Exp Psychol Hum Percept Perform, 32(6), 1436-1451.

Wassermann, E. M. (1998). Risk and safety of repetitive transcranial magnetic stimulation: report and suggested guidelines from the International Workshop on the Safety of Repetitive Transcranial Magnetic Stimulation, June 5-7, 1996. Electroencephalogr Clin Neurophysiol, 108(1), 1-16.

Woodward, T. S., Cairo, T. A., Ruff, C. C., Takane, Y., Hunter, M. A., \& Ngan, E. T. (2006). Functional connectivity reveals load dependent neural systems underlying encoding and maintenance in verbal working memory. Neuroscience, 139(1), 317-325. 


\section{Chapter 5 Visual evoked potentials under conditions of pre-stimulus occipital T'MS}
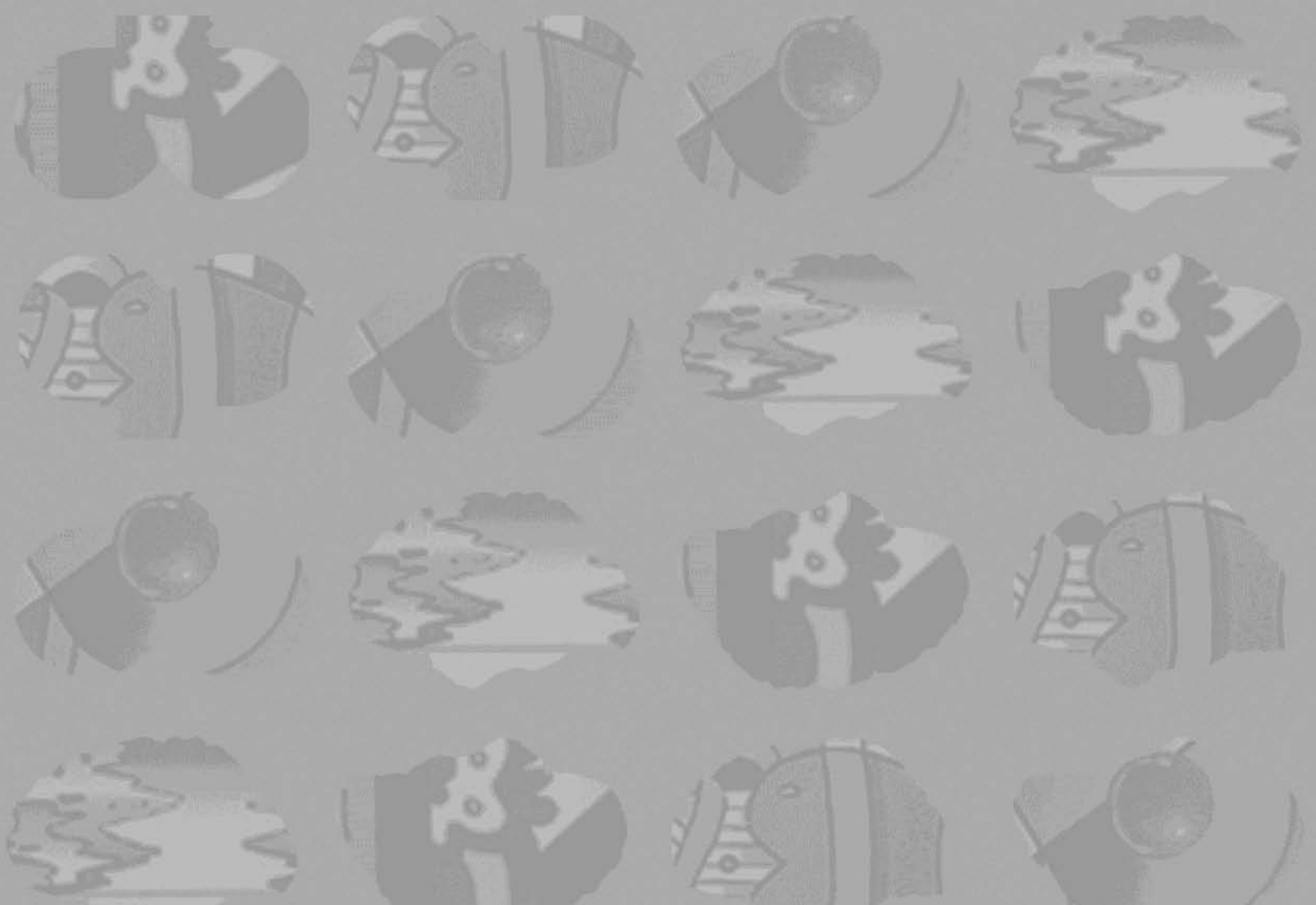


\section{ABSTRACT}

In the current study, we aimed to detect visual evoked potentials (VEPs) timelocked to the onset of a visual stimulus, when it was preceded by a single pulse of transcranial magnetic stimulation (TMS) over contralateral early visual cortex. The high-voltage TMS-induced artifact masks the small modulations due to the visual stimulus, making the retrieval of VEPs complex. First, we assessed two EEG-compatible TMS systems on the duration of the immediate TMS artifact. Based on system-dependent differences in the signal recovery latency, we selected one of the TMS systems for application in the second part of the study. In this part, we applied single pulses of TMS $\sim 50 \mathrm{~ms}$ prior to visual stimulusonset, while recording electroencephalography (EEG), and calculated the VEPs at different stages of data cleaning. TMS template subtraction reduced the signal to proportions in the order of standard VEPs, allowing the comparison of VEPs with and without prior TMS application. Analogues of classical VEPS components (P1, N180 and N200) were detected, although the earliest C1 component did not show in the VEPs because the EEG signal at these early latencies was still much distorted due to the TMS pulse. Data pruning through manual ICA component rejection further improved data quality, but mainly in the prestimulus domain. During the next stages of processing, the EEG data will be linked to behavioral responses and compared to conditions of post-stimulus occipital TMS. 


\section{INTRODUCTION}

In cognitive neuroscience, transcranial magnetic stimulation (TMS) is applied as a non-invasive brain interference technique that allows the temporal disruption of neural processing in healthy individuals through an intact skull. If TMS delivered to a given brain area causes a behavioral impairment, e.g. increased reaction times, the area is believed to be task-relevant and the neural activity within the area to bear a causal relation with the cognitive function under study. By these means, TMS has proven a valuable tool in mapping cognitive and perceptual functions onto the human cortex. However, the mere recording of behavioral reactions is not very informative with regards to the direct changes in neurophysiology underlying the behavioral TMS effects. Moreover, the influences of TMS on neural activity at distant, connected brain areas, and the way in which these contribute to the observed behavior remain concealed. The combination of TMS with neuroimaging methods therefore has become increasingly more established lately. Dependent on whether the research question benefits more from high temporal or spatial resolution, the concurrent application of electroencephalography (EEG) or functional magnetic resonance imaging (fMRI) are most common, respectively.

If the observed TMS effects show high temporal specificity, and the neural correlate of interest is therefore suspected to be best captured when measured with high temporal resolution, the additional acquisition of EEG data is the most obvious approach. EEG directly measures small potential differences on the scalp caused by the electrical signaling of large neuron populations, and it therefore has an almost instantaneous temporal resolution. However, the duration of the EEG artifact induced by the TMS pulse still limits the temporal resolution of the EEG in a multimodal set-up somewhat, because a short interval of data acquired immediately following the TMS pulse is lost, which is potentially challenging to any chronometric TMS experiment.

Of course the tiny voltage differences across the brain registered with EEG are severely disturbed by the strong magnetic field released in the form of a TMS pulse. The duration of a biphasic TMS pulse is less than $\sim 600 \mu$ s (Julkunen, et al., 2008; Litvak, et al., 2007), which is negligible given the usual sampling rate in EEG studies. If the immediate pulse release were the only source of distortion, just a single EEG sample would be contaminated by TMS-related artifact. Unfortunately, in the literature prolonged TMS artifacts have been reported (see Ilmoniemi \& Kicic, 2010). Note that we here consider anything in the EEG-signal that is not of interest to the research question and that can be said to be a consequence of magnetic pulse release to be artifactual. An immediate high-amplitude artifact with maximal deflections of several $\mathrm{mV}$ has been shown to be constrained to the first $6 \mathrm{~ms}$ post-TMS pulse (Veniero, Bortoletto, \& Miniussi, 2009), which is still entirely acceptable. But there are reports of still longer-lasting TMS-induced data contamination up to as much as several hundred milliseconds post-pulse (Ilmoniemi \& Kicic, 2010). 
There are many potential causes to this prolonged TMS artifact, both physical and physiological. Most likely, the eventually recorded EEG signal forms an interplay of multiple of these factors. Suggested physical causes of prolonged TMS artifact are eddy currents induced within the measuring electrodes (Veniero, et al., 2009), induced scalp charge (Julkunen, et al., 2008), and electrode movement caused by the TMS pulse (Virtanen, Ruohonen, Naatanen, \& Ilmoniemi, 1999). Muscle contraction, and auditory evoked potentials due to the clicking sound of TMS (Nikouline, Ruohonen, \& Ilmoniemi, 1999) are examples of suggested physiological artifact sources. Whatever the nature of the TMS artifact, in all reported cases, it is of much higher amplitude than the cortical signal of interest.

There are multiple ways of dealing with the TMS-induced artifact. In the earliest concurrent TMS-EEG studies, an online strategy was applied. EEG data acquisition was temporarily halted (clamping), to avoid permanent amplifier saturation caused by the immediate high-amplitude TMS-evoked signal (Ilmoniemi, et al., 1997; Virtanen, et al., 1999). This strategy proved successful in preventing amplifier saturation, but nevertheless did not rid the EEG signal of TMS-induced artifact altogether, and offline strategies to remove the residual artifacts were still required (Komssi, Kahkonen, \& Ilmoniemi, 2004; Litvak, et al., 2007). Newer TMS-compatible EEG systems allow the continued recording of EEG data without the need for sample-and-hold strategies (Bonato, Miniussi, \& Rossini, 2006; Ilmoniemi \& Kicic, 2010; Taylor, Walsh, \& Eimer, 2008; Thut, et al., 2003). In practice, the EEG signal within at least 5-10ms after TMS pulse release is still disregarded, because, during the immediate high-amplitude part of the TMS artifact, retrieval of the neural, low-amplitude EEG signal is virtually impossible.

The most straight-forward approach of dealing with the TMS-induced EEG artifact is just to ignore the artifactual data, and to only consider the signal starting from a time point after the TMS artifact has subsided (Dugue, Marque, \& VanRullen, 2011; Taylor, Walsh, \& Eimer, 2010). Typically, the data during this interval is replaced by interpolated data to allow subsequent data filtering without the introduction of ringing artifacts, which would further stretch the length of the affected EEG signal (Taylor, et al., 2008; Thut, et al., 2011). Obviously, these approaches require an accurate estimation of the period of data contamination, for example by the inclusion of an adequate control condition.

Instead of neglecting the TMS artifact, different methods of offline artifact removal have been explored until now. A couple of studies used subtraction logic as a means to clean the EEG signal from TMS-locked activity (Reichenbach, Whittingstall, \& Thielscher, 2011; Thut, et al., 2003). During TMS only trials, TMS pulses were applied to the experimental stimulation site, and the average EEG signal on these trials was subsequently subtracted from the averaged evoked responses on TMS trials which included an additional (visual) stimulus. This method of artifact removal has proven fairly successful in studies investigating the suppressive effects of occipital TMS (Reichenbach, et al., 2011; Thut, et al., 2003). 
Subsequently, computational ways of dealing with the TMS-induced artifacts have been suggested. Different filtering methods have been tested (Morbidi, et al., 2007), computational models based on independent component analyses (ICA; Korhonen et al., 2011) and source modeling (Litvak, et al., 2007) have been applied. All these methodologies have been tested on frontal stimulation sites only, and all have shown their merit in the removal of the reasonably large muscle artifact resulting from TMS stimulation at these locations. However, the question is whether these computational methods are successful in cleaning the EEG signal to a sufficient extent, when TMS is applied at a different cortical location. Event-related potentials (ERPs) typically are low amplitude modulations of the EEG signal in the order of microvolts, which can only be traced by averaging across large numbers of trials. Any residual TMS artifact of higher amplitude will mask these small evoked potentials, even if it is already much reduced compared to the high-voltage artifact which has a magnitude in the order of the millivolts.

The aim of the current study was twofold. First, two different TMS stimulator types were compared with regard to their suitability for concurrent EEG recording. They were specifically tested on the duration of the immediate highvoltage TMS-induced EEG artifact. On this basis, we selected one of the tested systems and applied this system in the second part of the study. In this part, a combination of TMS template subtraction and subsequent ICA component rejection was performed on a real dataset to assess this combined approach of TMS artifact removal. Visual evoked potentials (VEPs) were calculated after each of the processing steps and the results were compared to VEPs acquired without preceding occipital TMS.

\section{PART 1: SYSTEMS COMPARISON}

\section{MATERIALS AND METHODS}

\section{Head models}

The TMS-induced EEG artifact was measured on three different head models. The first model consisted of a Styrofoam dummy head. This model was used in a set-up identical to a measurement with a real individual, keeping the electrode cap lay-out and TMS coil orientation the same. Unfortunately, the noise levels in the signal obtained with this head model made the data incomparable to the data obtained on the other two surfaces, and these data are therefore not discussed in the remainder of the chapter. Secondly, the signal resulting from TMS stimulation of a Cucumis Melo (Galia melon) was tested, because the difference in dielectrical constant between skin and pulp of this species are comparable to the differences in dielectrical constant between 
human skin and grey matter (see Veniero et al., 2009 for details). Because of the relatively small size of the melon in comparison with a real head, we did not manage to acquire fairly noise-free signal in all electrodes, and we had to move the site of stimulation to a more frontal location (see also TMS Stimulation). Finally, recordings were performed on the real head of a healthy individual.

\section{TMS stimulation}

TMS artifacts of two different TMS stimulator brands were measured: 1) Magstim Rapid ${ }^{2}$ stimulator with a first generation figure-of-eight coil (double 70mm; The Magstim Company, Whitland, UK), and 2) the Magpro x100 stimulator with a MC-B70 figure-of-eight coil (Medtronic Functional Diagnostics A/S, Skovlunde, Denmark).

Biphasic TMS pulses of either high or low stimulation intensity were applied. For the Magpro system the high intensity was set to $80 \%$ maximum stimulator output, whereas for the Magstim system an intensity of $90 \%$ maximum stimulator output was chosen because of the lower maximal field strength achievable with the Magstim system. We deliberately tested at these very high intensities, because of our particular interest in occipital TMS on the EEG signal, and because TMS masking studies usually employ high intensity occipital stimulation (see Part 2). The low intensity conditions consisted of 10\% maximum stimulator output for both TMS systems. In contrast to the Magstim setup, the Magpro x100 stimulator offers the option to delay the stimulator recharge. We made use of this option and set the recharge delay to $1000 \mathrm{~ms}$.

The TMS coil was always held in horizontal position with the coil handle pointing towards the right. The melon was only subjected to high intensity stimulation. The stimulated electrode on the Styrofoam head model was 02. Because it proved difficult to stimulate this electrode on the melon surface, a different stimulation electrode was chosen here, namely Pz. On the real head, TMS was applied over 7 different electrode locations (01, 02, P03, P04, C3, C4 and $\mathrm{Cz}$ ). Per condition between 16 and 50 trials were collected. Inter-trial-interval jittered around $5000 \mathrm{~ms}$. The EEG data were acquired in three separate sessions. In each of these sessions both systems were used for TMS stimulation on a single model.

\section{EEG recording and analysis}

EEG data acquisition was performed with TMS-compatible EEG equipment (BrainAmp MR Plus amplifier; operating range $=3.227 \mu \mathrm{V}$; (BrainProducts $\mathrm{GmbH}$, Munich, Germany)). A 64-electrode TMS-compatible EEG cap ('Fast ' $n$ Easy', BrainProducts GmbH, Munich, Germany) was positioned on each of the three surfaces. Electrode positions on the cap are based on the international 10-20 system and are thus equidistant. The $\mathrm{Ag}-\mathrm{AgCl}$ electrodes are fixed in 
plastic sockets on the cap. Data acquisition was achieved through the BrainVision Recorder software (BrainProducts GmbH, Munich, Germany). Abrasive EEG-gel was applied to the electrodes to aid the conductivity of the signal. Contact impedance was reduced to $<\sim 5 \mathrm{kOhm}$. The data were collected with a sampling rate of $5 \mathrm{kHz}$ and no online filtering was performed during data acquisition. All recordings were referenced online to the $\mathrm{FCz}$ electrode. Offline, all raw data were epoched from -200 to $500 \mathrm{~ms}$ time-locked to TMS pulse-onset. The baseline as calculated on the $200 \mathrm{~ms}$ prior to TMS pulse onset was subsequently subtracted. Data processing was performed with BrainVision Analyzer 2.0 (BrainProducts GmbH, Munich, Germany), and Matlab R2007a (MathWorks, Natick, Massachusetts, U.S.A) software.

\section{RESULTS}

\section{Reproducibility}

The TMS artifact proved to be highly stable across trials, in shape, size and in duration. Figure 1 shows the TMS-locked EEG signal for 45 trials (cortical stimulation, stimulated electrode). Because no synchronization box was employed to perfectly align the TMS-pulse onset with EEG-sampling, there are minor offset differences. The use of an extremely high sampling rate (i.e. $5000 \mathrm{~Hz}$ ) partially compensates by limiting the maximal TMS latency difference to $0.2 \mathrm{~ms}$. Overall, the artifact shape is very reproducible, a pattern which we observed for all head models and all stimulator types. Therefore, we decided it was justifiable to further report on the TMS-induced artifacts averaged across trials.

\section{Duration}

The immediate high-voltage artifact as measured on the surface of a Galia melon lasted approximately $5.4 \mathrm{~ms}$. These data were acquired with the Magstim system and the data are therefore directly comparable to the results of Veniero et al. (2009). Using the same TMS system, these authors recorded an induced artifact that lasted maximally $5.6 \mathrm{~ms}$, and hence our data prove to be in line. Furthermore, the Magstim artifact shapes in both studies look highly similar (compare Figure 1 to figures 1, 2 and 4 in Veniero et al., 2009), and very stable in shape and duration across different stimulation intensities and stimulated surfaces (Figure 2; Panel 1), although we also replicated the additional deflections, at 6 and $8 \mathrm{~ms}$ post-pulse, which were specific to cortical stimulation.

Veniero et al. (2009) reported a second, low amplitude artifact some milliseconds after pulse onset, resulting from stimulator recharge. The latency of the recharge artifact proved dependent on stimulator type and stimulation intensity. Here, the low intensity condition did not reveal any recharge artifact. In 
the high intensity condition, a recharge artifact showed after $27.9 \mathrm{~ms}$ with a duration of $2.1 \mathrm{~ms}$ and a peak-to-peak amplitude of $47.5 \mu \mathrm{V}$ (see Figure 2; zoom). These values conflict with those of Veniero et al. (2009), because the authors reported a smaller recharge artifact with a stable amplitude of $\sim 12 \mu \mathrm{V}$, and a flexible latency predicted to be $\sim 60 \mathrm{~ms}$ post-pulse given the stimulator type and stimulation intensity employed in the current study.

Figure 3 also includes the TMS-locked EEG signal acquired with the Magpro system. Similar to the Magstim system, artifact duration remains rather short, at least for the low intensity conditions (see Intensity), as the main high-voltage TMS artifact seems to have subsided within $6 \mathrm{~ms}$. As we set a recharge delay to $1000 \mathrm{~ms}$ no recharge-related fluctuations of the EEG signal are apparent within the temporal scope of Figure 2.

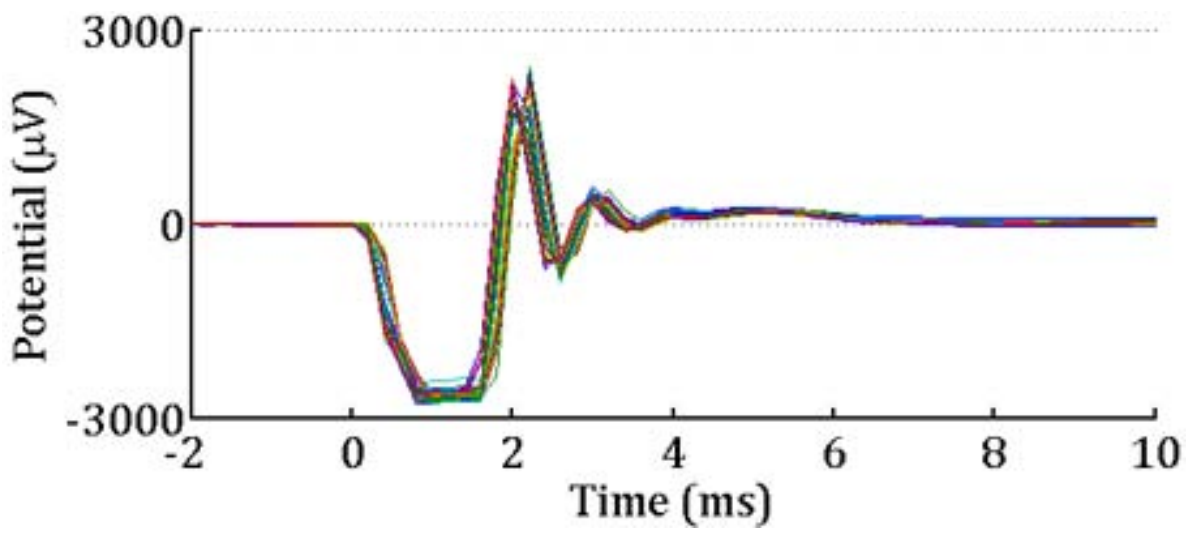

Figure 1. Shape of the immediate TMS artifact within the EEG signal. Lines represent the immediate EEG response locked to the onset of the TMS pulse on various trials $(n=45)$. The data are recorded from the electrode under the TMS coil (02), and were acquired on the head of a healthy volunteer.

\section{Intensity}

The Magstim and Magpro systems produce artifacts with a very similar shape and amplitude across intensities. The similarity in artifact magnitude is remarkable. TMS pulses were applied with intensities at the two extreme ends of the spectrum, and the physical properties of the pulse, i.e. magnetic field change and induced electric field, deviate to a large extent. But even following low intensity stimulation, large TMS-induced peaks in the EEG signal occur. This finding is particularly relevant, because placebo TMS coils do not shield the magnetic field altogether, but usually a magnetic field with the strength of a small percentage of the tuned intensity still passes through the coil casing. Hence, on the basis of these data, placebo TMS stimulation would be expected to also induce large EEG signal distortions. In a study, employing a Magstim Placebo 
coil as sham control, similar artifacts to those elicited with a standard TMS coil, were indeed detected (Bonato, et al., 2006).

However, the most important parameter to confine is artifact duration, in order to lose as little of temporal resolution as possible. Both systems show longer TMS-induced artifacts when stimulating with higher intensities, but the systems vary in their absolute artifact durations. The difference is particularly apparent for the Magpro system. The low intensity stimulation data return back to baseline immediately after the direct effect of the TMS pulse on the EEG signal has elapsed. In contrast, in the high intensity condition there is a drift in the signal for at least $30 \mathrm{~ms}$ post-pulse, and the signal does not completely return to baseline within $50 \mathrm{~ms}$. Inspection of the data beyond the interval depicted in Figure 2 reveals that the signal reaches baseline only $\sim 200 \mathrm{~ms}$ after the TMS pulse was delivered.
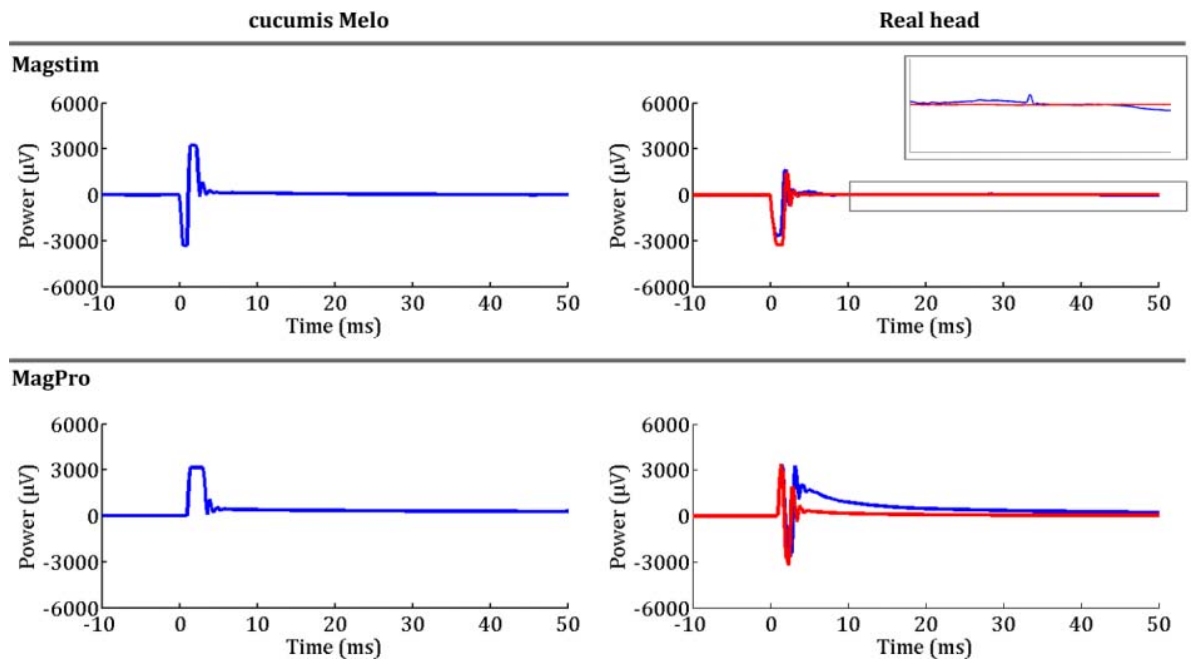

Figure 2. Overview of TMS artifacts for two different stimulator types and two different head models $(\mathrm{N}=16)$. Lines represent different TMS intensities (blue= high intensity; red=low intensity). The right top panel zooms in on the recharge artifact, which occurs $\sim 28 \mathrm{~ms}$ after pulse release. On the cucumis Melo (Galia melon) electrode Pz was stimulated. Real head data for electrode 02 are shown here.

\section{Conclusion}

Part 1 of the study consisted of an evaluation of two different TMS set-ups for EEG compatibility. Based on the outcome, the Magstim system was considered most suitable for the purpose of Part 2, which is adequate extraction of VEPs following single pulses of pre-stimulus TMS. Therefore, the temporal properties of the TMS-induced electrophysiological distortion were emphasized. Even though the Magpro system elegantly circumvents the problem of the recharge 
artifact by offering the option to delay stimulator recharge, the slow recovery of the signal after high intensity stimulation discouraged usage of the system in chronometric TMS designs, and we therefore selected the Magstim system to perform the TMS stimulation in the remainder of the study.

\section{PART2: ARTIFACT REMOVAL}

\section{MATERIALS AND METHODS}

\section{Participants}

After considering our results of Part 1, we decided to collect an experimental dataset with seven healthy participants (seven females; mean age 23 (range 2126)), with normal or corrected-to-normal visual ability. All participants were psychology students from Maastricht University, and they were financially compensated for participation. Before taking part, all participants filled out a health questionnaire to screen them for potential TMS risk factors (such as a history of epilepsy, drug use etc.). Only after approval by our medical supervisor were they invited for participation. All participants signed a written informed consent at the start of each session. The study was approved by the Medical Ethics Committee of the University Medical Center, Maastricht, the Netherlands.

\section{Procedure}

After the EEG-cap had been mounted on the head, the participant was comfortably positioned in front of a computer monitor at an eye-to-screen distance of $57 \mathrm{~cm}$. The head of the participant was stabilized in a chin-rest, and the participant was provided with ear plugs for hearing protection.

The session commenced with a short training run to acquaint the participant with the visual discrimination task. The participant was required to judge whether a Landolt $C$ stimulus, briefly presented in the left lower visual field at an eccentricity of $4^{\circ}$ (size of $1.0 \times 1.0^{\circ}$ ) visual angle, was mirror-imaged or not. In other words, they were to judge whether the gap was on the left or right side of the stimulus. Via a second button press, participants indicated on a 4-point scale whether they had a conscious percept of the visual stimulus ("I saw the stimulus really well"; "I saw the stimulus fairly well"; "I did not see the stimulus so well"; "I did not see the stimulus at all"). The training run consisted of two blocks of 16 trials each. During training, participants were given online feedback on their discrimination performance.

Thereafter, participants performed four experimental runs. Halfway through the session, a half hour break was given to participants to reduce fati- 
gue. The first and second half of the session were identical and consisted both of a baseline (No TMS) run and a TMS run. At the end of the session, electrode positions were localized by means of the Zebris hardware and accompanying ElGuide software package (Zebris Medical GmbH, Isny im Allgäu, Germany).

Two blocks of 30 trials without feedback or TMS were collected during the No TMS runs. The TMS runs consisted of 240 trials each, divided over 15 blocks of 16 trials. A single TMS pulse was applied over occipital cortex (see TMS Stimulation), which in 50 percent of the cases preceded the visual stimulus (prestimulus TMS) and in the other 50 percent followed it (post-stimulus TMS).

In between the experimental runs, short sequences of 25 TMS pulses (TMS ${ }_{\text {only }}$ ) separated by 4-6 seconds were delivered to EVC while the concomitant EEG signal was recorded. During these 'template' runs, the participants' only task was to maintain fixation.

\section{TMS stimulation}

Based on the results of Part 1, the Magstim system (Rapid ${ }^{2}$ stimulator and first generation 70mm double coil; The Magstim Company, Whitland, UK) was selected as the most optimal system, rendering the most stable artifacts of shortest duration, and it was hence employed in the second part of the study as well.

The individual TMS target site was based on fMRI-data acquired with a visual localizer in an earlier session $(128 \times 128$ matrix, $2 \mathrm{~mm}$ isovoxel, TE $=30 \mathrm{~ms}$, $\mathrm{TR}=2000 \mathrm{~ms}$ ). While participants were scanned, they were presented with alternating blocks of colored, flickering circles in left and right lower visual field, or rings adjacent to the outer contours of the circle stimulus. The position of the stimuli was chosen such that the inner circle of the localizer overlapped with the location of visual stimulation during the later TMS-EEG session. Full-model General Linear Model analysis was performed in each individual (Brainvoyager QX v2.3.0, BrainInnovation, Maastricht, the Netherlands). The left circle and ring predictors were subsequently contrasted, resulting in a map of voxels in right occipital cortex, which showed a higher activation for the center of the circle compared to its borders. In each individual, the region that survived the contrast, and that was closest to the calcarine sulcus was selected as TMS target site (see Figure 3). During the experimental TMS-EEG session, neuronavigation (TMS Neuronavigator, BrainInnovation, Maastricht, the Netherlands) was employed to accurately position the TMS coil over the selected target site. In practice, this meant that in most cases the TMS coil was positioned approximately in between electrodes $\mathrm{O2}, \mathrm{Oz}$ and $\mathrm{PO}$. 


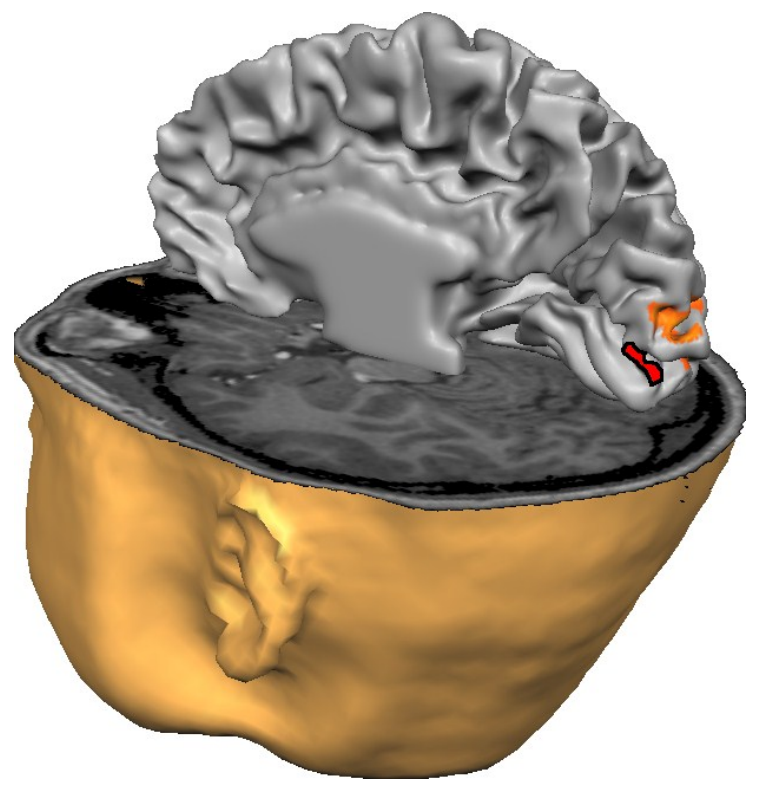

Figure 3. TMS stimulation site in a single subject. The red piece of cortex represents the stimulation site selected in this participant. Orange patches represent other brain areas activated by the stimulus localizer.

Single TMS pulses were delivered to EVC at one of two time windows: in the pre-stimulus, or in the post-stimulus temporal domain. EEG signal processing, as described in the next section, was only performed on pre-stimulus TMS trials to get a clear estimation whether the prolonged TMS artifact interferes with the evoked response to a later visual stimulus, and if so, in which time frame this interference is resolved. Time windows were selected on the basis of a preexperimental TMS session. In this session, participants performed the experimental task (see Procedure), while they received single pulses of occipital TMS across a range of pre-stimulus time windows (16 trials/time window). Reaction times (RTs), accuracy and awareness were inspected in each individual and the combined behavioral pattern of these three measures resulted in the selection of the optimal TMS suppression time window per individual (see Figure 4). In five participants, the selected time window of TMS application was $-50 \mathrm{~ms}$, in one participant $-45 \mathrm{~ms}$ and in one participant $-30 \mathrm{~ms}$. This approach was taken because, at a later stage, the EEG responses per level of visibility will be investigated. Statistical analyses on the data demonstrated that our visual stimulus was indeed suppressed by pre-stimulus TMS. Separate univariate GLM analyses revealed main effects of pre-stimulus TMS on all three measured variables. Post hoc comparisons of No TMS to the different TMS time windows revealed significant differences for the $-30,-45,-50$ and -55 time windows on RTs $(\mathrm{p}<.05)$. Accuracy was significantly impaired when TMS was applied $50 \mathrm{~ms}$ prior to the visual stimulus ( $\mathrm{p}<.05)$, and subjective awareness was significantly reduced across all pre-stimulus time windows $(\mathrm{p}<.05)$. 
The used TMS intensity was identical to that used in Part 1 (90\% maximum stimulator output), unless muscle twitches or other discomforts to the participants were noticed. In these cases, the highest endurable TMS intensity was used for stimulation (average intensity $=88.5 \%$ ).

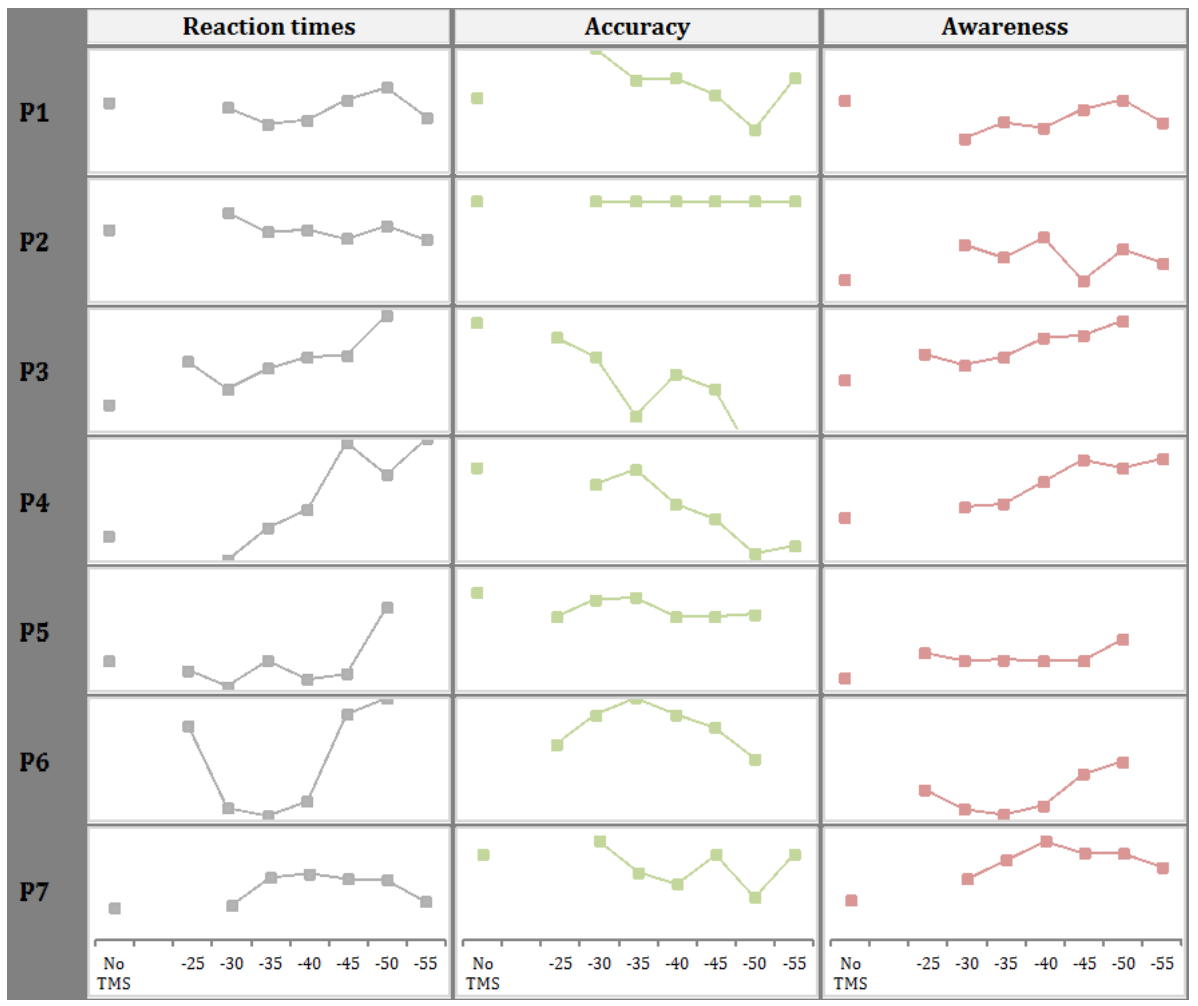

Figure 4. Behavioral data per participant for the pre-experimental TMS session. Left column represents reaction times (Y-axis spans $400 \mathrm{~ms}$ ). Middle column represents accuracy (Y-axis in all graphs runs from 30 to $100 \%$ correct responses). Right column represents awareness ratings (Yaxis runs from 1 ('perfect awareness') to 4 ('no awareness')). Based on these three measures, we selected a pre-stimulus time window per individual at which visual suppression was optimal.

\section{EEG recording and analyses}

For details on EEG equipment and recording, see the section on EEG recording in Part 1. Off-line analysis of the No TMS trials commenced with re-referencing to common electrode, and re-sampling to $500 \mathrm{~Hz}$. Data were filtered with a band-pass filter of $2-100 \mathrm{~Hz}$, and the data were epoched around visual stimulus 
A)
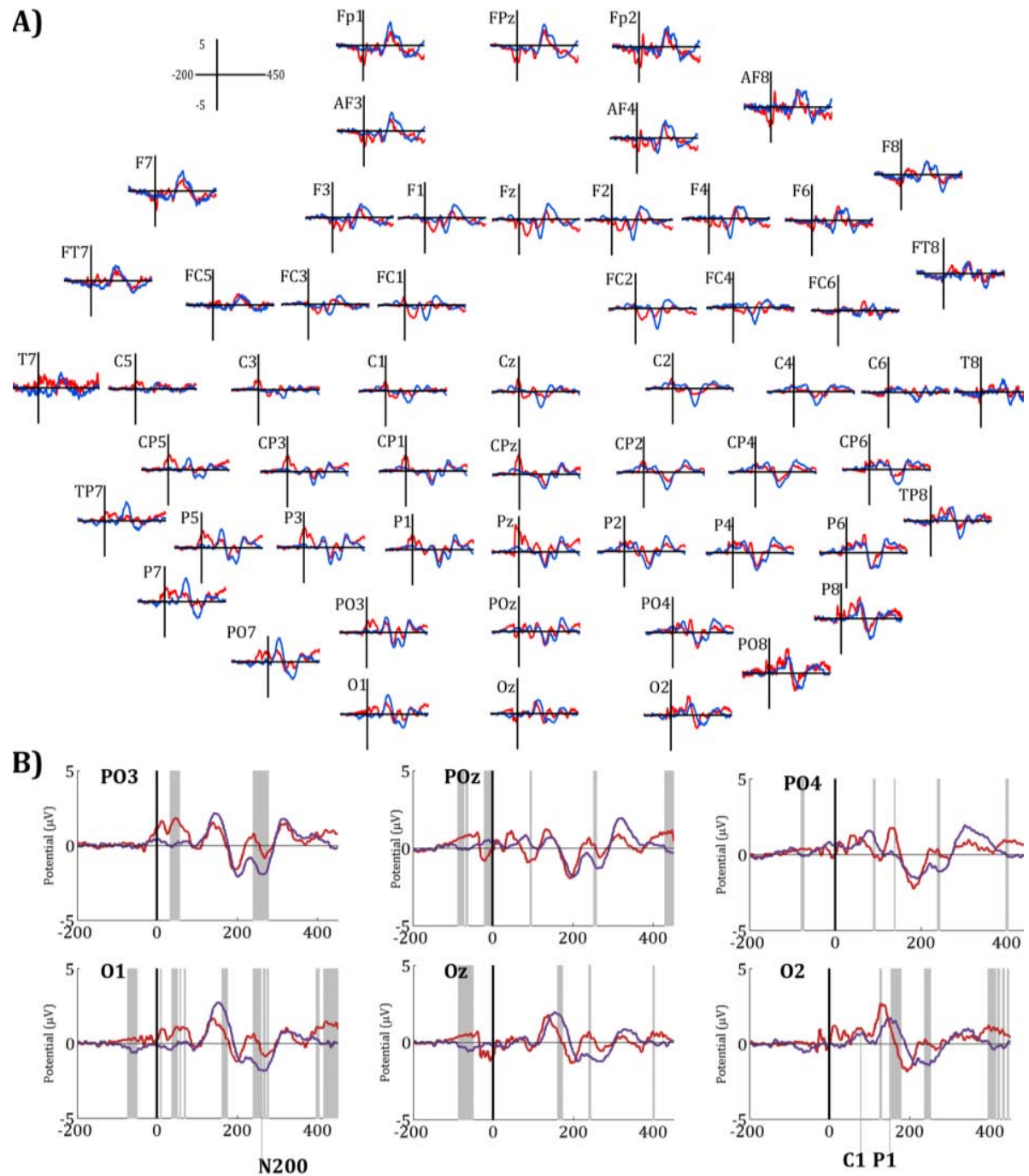

Figure 5. A) Grand averages of 57 electrodes which rendered proper signal in all seven subjects. The vertical axes represent visual stimulus onset. Blue lines represent the averaged recorded signal on trials without TMS (No TMS), and red lines on trials with TMS. B) Zoom on six (parieto)occipital electrodes: central $(\mathrm{Oz}, \mathrm{POz})$, contralateral $(02, \mathrm{P0} 4)$ and ipsilateral $(01, \mathrm{PO})$ to the side of visual stimulation (lower left visual field). Grey shades represent intervals at which the two conditions are significantly different. 
onset by -200 to $450 \mathrm{~ms}$. Baselines were calculated on the interval -200 to -150 ms. For each experimental TMS run, offline data processing first involved TMS template subtraction. Data from the two most proximal $\mathrm{TMS}_{\text {only }}$ runs were epoched around TMS pulse onset (-200 to $450 \mathrm{~ms})$ and subsequently averaged and baseline corrected in order to create a template of TMS evoked EEG-signal in absence of visual stimulation. This template was calculated and subsequently subtracted channel wise from the experimental data on a single trial basis. The data in the $15 \mathrm{~ms}$ immediate following the TMS pulse were interpolated. Data were then downsampled to $500 \mathrm{~Hz}$, re-referenced to common electrode, and band-pass filtered $(2-100 \mathrm{~Hz})$. Epochs were created from -200 to $450 \mathrm{~ms}$ timelocked to visual stimulus onset. The baseline as calculated from the -200 to $150 \mathrm{~ms}$ interval was subsequently subtracted.

The final analysis step consisted of data decomposition in multiple independent components using the logistic infomax ICA of Bell \& Sejnowski (1995). The number of resultant independent components equaled the number of input channels. On the basis of component ERPs and topographic representations, seven to nine artifactual components were selected and removed from the dataset. No TMS data were cleaned from eye blink artifacts using the same approach (maximal rejection of two artifactual components). Visual evoked potentials (VEPs) were subsequently calculated by averaging the pruned EEG data time-locked to visual stimulus onset.

To evaluate whether the VEPs calculated on the cleaned EEG data resemble standard VEPs, pairwise t-tests with were performed comparing the two conditions for each time point, both pre- and post-stimulus. Critical $\alpha$-level was set to $5 \%$, and p-values were not corrected for multiple comparisons. We deliberately performed very lenient statistical tests, because we particularly wanted to prevent TypeII errors (i.e. not detecting an actual difference) rather than TypeI errors. A similar comparison was performed for the VEPs resultant from the data after TMS template subtraction, and the VEPs resultant from the data further pruned by means of ICA. This analysis served to evaluate the added value of ICA pruning on top of EEG data cleaning with TMS template subtraction in isolation.

All offline analyses were performed with Matlab R2007a (MathWorks, Natick, Massachusetts, U.S.A) software, and the EEGLab Matlab Toolbox v.8.0 (MathWorks, Natick, Massachusetts, U.S.A (Delorme \& Makeig, 2004)).

\section{RESULTS}

The visual stimulus successfully evoked stable electrophysiological responses (see Figure 5.A, blue lines). The time course of the VEPs showed a first positive deflection in contralateral (right) occipital channels with an onset latency of $55 \mathrm{~ms}$ and a peak latency of $80 \mathrm{~ms}$, corresponding to the classic $\mathrm{C} 1$ component. The polarity of this component has been shown to depend on the position of the 


\section{A) C1: $60-85 \mathrm{~ms}$}

TMS

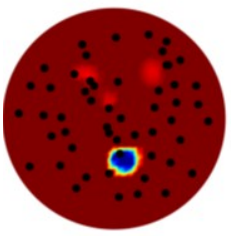

\section{TMS-template}

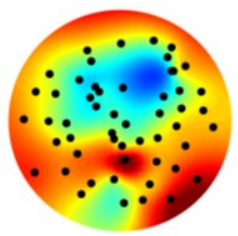

TMS-template (ICA)

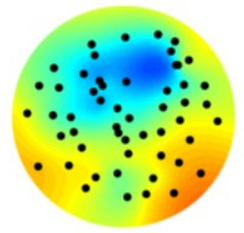

\section{No TMS}

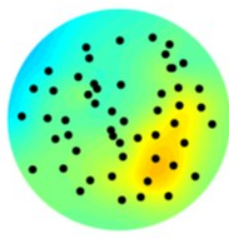

B) P1: 130-150ms

TMS

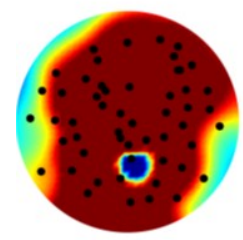

C) N180-N200: 170-210ms

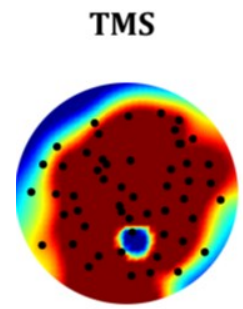

TMS-template

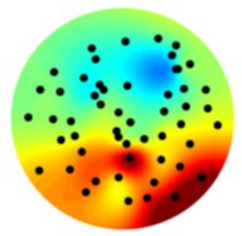

TMS-template

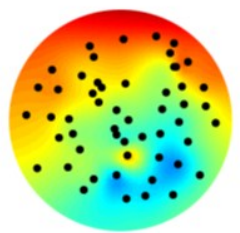

TMS-template (ICA)

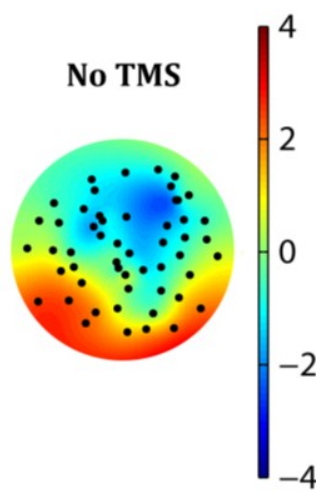

No TMS
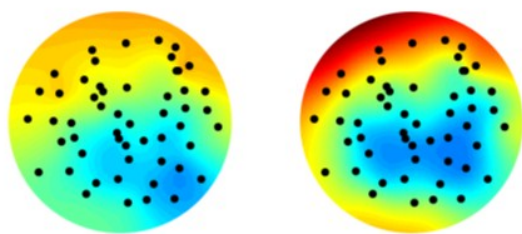

Figure 6. Topographies for three visual components: C1, P1 and N200. Black dots represent (average) channel locations. The left three panels all show data acquired in the TMS run. The outer left column are the topographies of the raw data. The inner left column contains topographies after TMS template subtraction, and the inner right column after additional data pruning with ICA. The far right column contains topographies of these classical VEP components in the absence of TMS application. Colors code for potential differences relative to baseline in $\mu \mathrm{V}$.

visual stimulus (lower versus upper visual field). In cases of lower visual field stimulation, the $\mathrm{C} 1$ turns out positive. A second positive component (onset latency $110 \mathrm{~ms}$, peak latency $150 \mathrm{~ms}$ ) occurred bilaterally over (parieto-) occipital sites, and corresponds to the P1 component. In the same time period right lateralized frontocentral negativity emerged (onset latency $\sim 115 \mathrm{~ms}$, peak latency $\sim 155 \mathrm{~ms}$ ). Finally, we find a bilateral negativity at parieto-occipital 

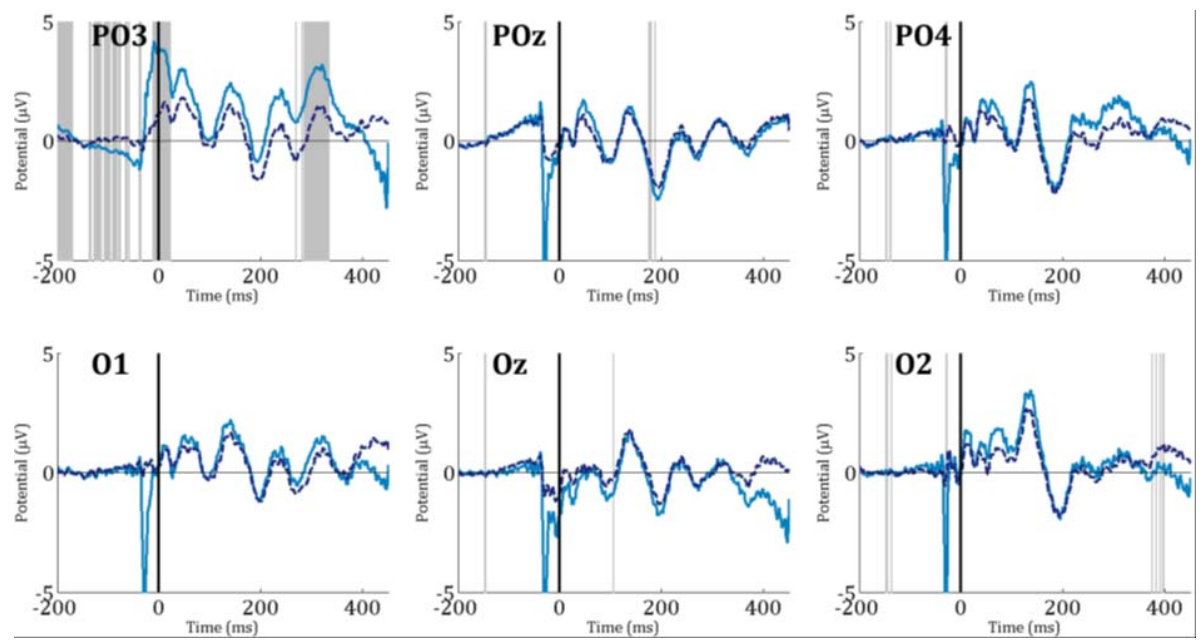

Figure 7. Evoked responses of six (parieto)-occipital electrodes: central (Oz, POz), contralateral (02, P04) and ipsilateral (01, P03) to the visual stimulation (lower left visual field). Lines represent analysis stages: solid lines are calculated evoked responses after the data are cleaned by means of template subtraction; dotted lines are calculated evoked responses after additional ICA pruning. Grey shades represent intervals at which the potential difference between the two conditions is significantly different.

channels (onset latency $\sim 175 \mathrm{~ms}$, peak latency $\sim 205 \mathrm{~ms}$ ), which is referred to as N180-N200 (for a general overview of VEPs and their neural sources, see Di Russo, Martinez, Sereno, Pitzalis, \& Hillyard, 2002).

The first, important point to make about the TMS results is that, after EEG data preprocessing, the variance in the electrophysiological signal locked to visual stimulus-onset is of a magnitude comparable to the actual VEPs under regular circumstances. When comparing the topographies in Figure 6 of uncorrected TMS-EEG data to the other two TMS preprocessing stages, it becomes clear that no stimulus-locked modulation appears in the raw data because the overall electrophysiological signal is still elevated compared to baseline, and to such an extent that the stimulus-locked responses are invisible.

The difference in VEPs between the completely pre-processed (template subtraction + ICA pruning) TMS data and the No TMS data is variable across the scalp (Figure 5.A), but visual inspection reveals corresponding waveforms, at least at contralateral posterior scalp locations. The first $100 \mathrm{~ms}$ after stimulus onset nevertheless remain rather noisy. The topographies show large frontal negativity in this time frame, which most probably reflects the complementary pole to the posterior positivity resulting from the TMS pulse. In Figure 5.B, the time points at which the TMS and No TMS conditions are significantly different are indicated by grey shading. In the pre-stimulus domain, significant differences occur due to the residual signal distortions in the TMS condition, which were not removed by template subtraction. The more interesting effects 
occur in the post-stimulus domain. VEP component peaks of posterior channels are larger over the right hemisphere, thus contralateral to visual stimulation and ipsilateral to TMS stimulation. The left hemisphere shows the reversed pattern with enlarged P1-component peaks for visual stimuli not preceded by TMS. Furthermore, a latency difference can explain part of the results for the right hemisphere, as P1 and N180-200 peak 20ms earlier on TMS compared to No TMS trials.

Finally, the added value of manual component rejection following template subtraction was assessed. The results are presented in Figure 7. Only channel PO3 showed substantial signal differences after ICA pruning, although mainly in the pre-stimulus domain. The later interval of significant amplitude difference (latency $\sim 325 \mathrm{~ms}$ ) can be explained by a reduction in VEP intercept, which again results from the removal of TMS noise in the pre-stimulus domain. Even if statistically insignificant, the effect of ICA pruning on the immediate TMS artifact is apparent in all six occipital electrodes as the sharp spike in the VEPs $\sim 35 \mathrm{~ms}$ prior to stimulus onset has disappeared.

\section{DISCUSSION}

In Part 1 of the study, two TMS systems were evaluated with regard to the duration of the TMS artifact they produce in the EEG data. In all the testing conditions, the immediate high-voltage TMS artifact was high reproducible across trials. The TMS-induced EEG artifact had been investigated before (Veniero, et al., 2009), though our conclusions are not altogether similar. Here, we replicate the shape and the duration of the main electrode-skin artifact, which was $<\sim 6$ ms. The delayed recharge artifact occurred $\sim 30 \mathrm{~ms}$ after high intensity stimulation, which is $\sim 30 \mathrm{~ms}$ earlier than the predicted latency on the basis of the previous study. In comparison, the Magpro system does not suffer from a recharge artifact at all, as the stimulator offers the additional option to delay recharge with a modifiable amount of time. We set the delay to $1000 \mathrm{~ms}$, and indeed no recharge artifact surfaced in the EEG data. However, there is an even larger concern with regard to the Magpro system, namely the slow return of the EEG signal to baseline after high intensity TMS stimulation.

Earlier studies reported that the duration of the high-voltage artifact is not dependent on stimulation intensity, even if the amplitude of the EEG signal is enlarged with higher intensities (Litvak, et al., 2007; Veniero, et al., 2009). Indeed, this seems to be the case for the Magstim system, but not for the Magpro system. The unstable duration of EEG data contamination across TMS intensities might prove problematic for chronometric TMS designs comparing conditions of variable pulse intensity. Overall, we concluded that the Magstim system appears most suitable for high resolution on-line TMS-EEG experiments.

In the second part of the study, VEPs in response to small, briefly presented visual stimuli were investigated, and the effect of a pre-stimulus TMS pulse on VEPs was assessed. The extraction of interpretable VEPs from the EEG signal 
when a TMS pulse is released in close temporal proximity to the visual stimulus proved challenging. But after the creation and subtraction of a TMS 'template', which models the typical EEG response to a TMS pulse, fluctuations in the EEG signal were reduced to the VEP order of magnitude. The early post-stimulus EEG signal still proved to be affected by the TMS pulse, though. Pruning of the data by rejection of artifactual ICA components did not lead to much improvement in this respect. The bilateral P1 and the N180-200 component were retrieved from the data successfully though. Topographically specific differences in their amplitude and peak latencies were detected when comparing the data to standard VEPs without preceding TMS.

The forward shift of contralateral occipital VEPs could reflect a cortical response to TMS-induced phosphenes. Phosphenes are experiences of vision in the absence of physical light, and these sensations can be induced through stimulation of early visual cortex. If these visual experiences evoke cortical responses similar to normal VEPs, the components in our TMS data are potentially time-locked to phosphene-onset rather than visual stimulus-onset. On the basis of the current dataset, we cannot exclude this option. Electrophysiological investigations into the neural responses to phosphene perception have been conducted (Dugue, et al., 2011; Taylor, et al., 2010). Unfortunately, these studies did not attempt to rid their electrophysiological data of prolonged TMS-induced artifacts. Therefore, the absolute response profile to TMS-induced phosphene perception remains unknown.

Taylor et al. (2010) compared the TMS-evoked potentials (TEPs) to TMS pulses that successfully induced phosphenes versus TMS pulses that did not result in the phosphene perception. TMS intensities were individually determined so that on average in 50 percent of the cases participants would experience phosphenes. Thus, all physical parameters across the conditions of interest were exactly identical. The TEPs of both trial types started to deviate approximately $160 \mathrm{~ms}$ after TMS pulse onset and the authors therefore concluded that recurrent posterior activity constitutes the conscious perception of a phosphene. Because the authors were interested in the modulation of TEPs by phosphene perception, the potential presence of residual artifact (demonstrated in the TEPs to control site TMS) became less harmful. Of course, the success of this strategy depends on the relative amplitude difference between the TMS-induced artifact and the EEG response under investigation. As we have shown here, template subtraction successfully reduces the amplitude of the EEG signal in the time period following the TMS pulse, and it thus allows adequate statistical comparison of the response modulations resulting from experimental manipulations.

A major caveat of all EEG data cleaning methods is that some of the neurophysiological consequences of TMS are removed together with the unwelcome artifactual data. This problem is inherent to the combination of TMS with any research method, data quality of which suffers from TMS pulse release. Because both physiological and artifactual TMS effects are dependent on, and thus time-locked to, pulse-onset, these two elements cannot be teased 
apart. Investigating the modulation of the EEG signal over different experimental conditions is the adequate work-around here. Even if some of the relevant neurophysiological data is eliminated together with the irrelevant TMSinduced artifact, this is unproblematic as long as the factor is kept constant over experimental conditions.

An example of an unresolved matter, which solution might lie in a similar approach, is the neurophysiology underlying post- and pre-stimulus TMS masking. TMS masking refers to visual suppression occurring as a consequence of occipital TMS. Amassian et al. (1989) were the first to show TMS masking in a chronometric TMS study, and they concluded that (early) visual cortex is relevant for visual discrimination particularly $80-100 \mathrm{~ms}$ after the onset of the visual display, a result that has been replicated on numerous occasions since (Beckers \& Homberg, 1991; Beckers \& Zeki, 1995; Camprodon, Zohary, Brodbeck, \& Pascual-Leone, 2009; Corthout, Uttl, Juan, Hallett, \& Cowey, 2000; Corthout, Uttl, Walsh, Hallett, \& Cowey, 1999; Corthout, Uttl, Ziemann, Cowey, \& Hallett, 1999; de Graaf, Cornelsen, Jacobs, \& Sack, 2011; Jacobs, Goebel, \& Sack, 2012; Overgaard, Nielsen, \& Fuglsang-Frederiksen, 2004; Sack, van der Mark, Schuhmann, Schwarzbach, \& Goebel, 2009). Later studies revealed that occipital TMS prior to stimulus onset (pre-stimulus TMS) also has a detrimental effect on visual perception (Beckers \& Homberg, 1991; Corthout, et al., 2000; de Graaf, et al., 2011; Jacobs, et al., 2012; Laycock, Crewther, Fitzgerald, \& Crewther, 2007). We recently demonstrated that this pre-stimulus TMS masking is of neural origin (see Chapter 2 or Jacobs et al., 2012) by falsifying alternative accounts attributing it either to eye blinking, or other non-specific TMS effects (Beckers \& Homberg, 1991; Corthout, et al., 2000; Sack, Kohler, Linden, Goebel, \& Muckli, 2006). However, we could not draw any definite conclusion concerning the related neural mechanisms, because our study lacked the neurophysiological data required to do so.

Reichenbach et al. (2011) applied occipital TMS pulses at different stimulusonset asynchronies time-locked to a flickering checkerboard, while concurrently recording EEG. The averaged VEPs showed a systematic enhancement of the P1 component from TMS to No TMS trials. This P1 enhancement gradually increased with stimulation intensity, but saturated at those TMS intensities that caused a severe behavioral impairment on a visual recognition task. The authors interpreted their findings as a reflection of the robustness of the signal in early occipital areas to noise inflicted for example by a TMS pulse. Thut et al. (2003) also reported TMS-related enhancement of VEPs, but in this case of the more frontal N150 component. Together, these findings contradict the conclusion of Harris, Clifford \& Miniussi (2008) that TMS exerts its neural effect through signal suppression rather than through noise induction. However, this conclusion was based on the behavioral effects of post-stimulus occipital TMS as acquired in a psychophysical framework, and, as Reichenbach et al. (2011) rightfully point out, the functional consequences of TMS might not directly transfer to the underlying neurophysiology in a one-to-one fashion. Moreover, the suppressive effects of pre- and post-stimulus TMS most likely have a devi- 
ant neural signature. Here, pre- and post-stimulus TMS pulses were applied to occipital cortex at titrated time windows in order to induce a maximal suppressive effect on visual perception. Because we found that our method of TMS template subtraction rendered interpretable VEPs, we plan follow-up analyses of the current dataset that will link the detected VEPs to behavior. We particularly hope to unravel the different neurophysiological responses to perceived versus unperceived visual stimuli. Furthermore, similar analyses will be performed on the post-stimulus TMS runs and these data will be contrasted with the physiological profile underlying pre-stimulus TMS masking.

\section{ACKNOWLEDGMENTS}

The research leading to these results has received funding from the Netherlands Organization for Scientific Research (NWO; grant number 400-07-048 to A.T.), and the European Research Council under the European Union's Seventh Framework Programme (FP7/2007-2013)/ERC Grant agreement $n^{\circ}$ [263472]).

We thank our medical supervisor Cees van Leeuwen, and our independent physician Martin van Boxtel. We also thank Sonja Cornelsen for discussions on the experimental design, Lars Hausfeld for assistance with EEG data preprocessing and Tahnée Engelen for assistance during data acquisition. 


\section{REFERENCES}

Amassian, V. E., Cracco, R. Q., Maccabee, P. J., Cracco, J. B., Rudell, A., \& Eberle, L. (1989). Suppression of visual perception by magnetic coil stimulation of human occipital cortex. Electroencephalogr Clin Neurophysiol, 74(6), 458-462.

Beckers, G., \& Homberg, V. (1991). Impairment of visual perception and visual short term memory scanning by transcranial magnetic stimulation of occipital cortex. Exp Brain Res, 87(2), 421432.

Beckers, G., \& Zeki, S. (1995). The consequences of inactivating areas V1 and V5 on visual motion perception. Brain, 118 ( Pt 1), 49-60.

Bell, A. J., \& Sejnowski, T. J. (1995). An information-maximization approach to blind separation and blind deconvolution. Neural Comput, 7(6), 1129-1159.

Bonato, C., Miniussi, C., \& Rossini, P. M. (2006). Transcranial magnetic stimulation and cortical evoked potentials: a TMS/EEG co-registration study. Clin Neurophysiol, 117(8), 1699-1707.

Camprodon, J. A., Zohary, E., Brodbeck, V., \& Pascual-Leone, A. (2009). Two phases of V1 activity for visual recognition of natural images. J Cogn Neurosci, 22(6), 1262-1269.

Corthout, E., Uttl, B., Juan, C. H., Hallett, M., \& Cowey, A. (2000). Suppression of vision by transcranial magnetic stimulation: a third mechanism. Neuroreport, 11(11), 2345-2349.

Corthout, E., Uttl, B., Walsh, V., Hallett, M., \& Cowey, A. (1999). Timing of activity in early visual cortex as revealed by transcranial magnetic stimulation. Neuroreport, 10(12), 2631-2634.

Corthout, E., Uttl, B., Ziemann, U., Cowey, A., \& Hallett, M. (1999). Two periods of processing in the (circum)striate visual cortex as revealed by transcranial magnetic stimulation. Neuropsychologia, 37(2), 137-145.

de Graaf, T. A., Cornelsen, S., Jacobs, C., \& Sack, A. T. (2011). TMS effects on subjective and objective measures of vision: Stimulation intensity and pre- versus post-stimulus masking. Conscious Cogn, 20[4], 1244-1255.

Delorme, A., \& Makeig, S. (2004). EEGLAB: an open source toolbox for analysis of single-trial EEG dynamics including independent component analysis. J Neurosci Methods, 134(1), 9-21.

Di Russo, F., Martinez, A., Sereno, M. I., Pitzalis, S., \& Hillyard, S. A. (2002). Cortical sources of the early components of the visual evoked potential. Hum Brain Mapp, 15(2), 95-111.

Dugue, L., Marque, P., \& VanRullen, R. (2011). The phase of ongoing oscillations mediates the causal relation between brain excitation and visual perception. J Neurosci, 31(33), 11889-11893.

Harris, J. A., Clifford, C. W., \& Miniussi, C. (2008). The functional effect of transcranial magnetic stimulation: signal suppression or neural noise generation? J Cogn Neurosci, 20(4), 734-740.

Ilmoniemi, R. J., \& Kicic, D. (2010). Methodology for combined TMS and EEG. Brain Topogr, 22(4), 233-248.

Ilmoniemi, R. J., Virtanen, J., Ruohonen, J., Karhu, J., Aronen, H. J., Naatanen, R., et al. (1997). Neuronal responses to magnetic stimulation reveal cortical reactivity and connectivity. Neuroreport, 8(16), 3537-3540.

Jacobs, C., Goebel, R., \& Sack, A. T. (2012). Visual awareness suppression by pre-stimulus brain stimulation; a neural effect. Neuroimage, 59[1], 616-624.

Julkunen, P., Paakkonen, A., Hukkanen, T., Kononen, M., Tiihonen, P., Vanhatalo, S., et al. (2008). Efficient reduction of stimulus artefact in TMS-EEG by epithelial short-circuiting by minipunctures. Clin Neurophysiol, 119(2), 475-481.

Komssi, S., Kahkonen, S., \& Ilmoniemi, R. J. (2004). The effect of stimulus intensity on brain responses evoked by transcranial magnetic stimulation. Hum Brain Mapp, 21(3), 154-164.

Korhonen, R. J., Hernandez-Pavon, J. C., Metsomaa, J., Maki, H., Ilmoniemi, R. J., \& Sarvas, J. (2011). Removal of large muscle artifacts from transcranial magnetic stimulation-evoked EEG by independent component analysis. Med Biol Eng Comput, 49(4), 397-407.

Laycock, R., Crewther, D. P., Fitzgerald, P. B., \& Crewther, S. G. (2007). Evidence for fast signals and later processing in human V1/V2 and V5/MT+: A TMS study of motion perception. J Neurophysiol, 98(3), 1253-1262.

Litvak, V., Komssi, S., Scherg, M., Hoechstetter, K., Classen, J., Zaaroor, M., et al. (2007). Artifact correction and source analysis of early electroencephalographic responses evoked by transcranial magnetic stimulation over primary motor cortex. Neuroimage, 37(1), 56-70. 
Morbidi, F., Garulli, A., Prattichizzo, D., Rizzo, C., Manganotti, P., \& Rossi, S. (2007). Off-line removal of TMS-induced artifacts on human electroencephalography by Kalman filter. J Neurosci Methods, 162(1-2), 293-302.

Nikouline, V., Ruohonen, J., \& Ilmoniemi, R. J. (1999). The role of the coil click in TMS assessed with simultaneous EEG. Clin Neurophysiol, 110(8), 1325-1328.

Overgaard, M., Nielsen, J. F., \& Fuglsang-Frederiksen, A. (2004). A TMS study of the ventral projections from V1 with implications for the finding of neural correlates of consciousness. Brain Cogn, 54(1), 58-64.

Reichenbach, A., Whittingstall, K., \& Thielscher, A. (2011). Effects of transcranial magnetic stimulation on visual evoked potentials in a visual suppression task. Neuroimage, 54(2), 13751384.

Sack, A. T., Kohler, A., Linden, D. E., Goebel, R., \& Muckli, L. (2006). The temporal characteristics of motion processing in hMT/V5+: combining fMRI and neuronavigated TMS. Neuroimage, 29(4), 1326-1335.

Sack, A. T., van der Mark, S., Schuhmann, T., Schwarzbach, J., \& Goebel, R. (2009). Symbolic action priming relies on intact neural transmission along the retino-geniculo-striate pathway. Neuroimage, 44(1), 284-293.

Taylor, P. C., Walsh, V., \& Eimer, M. (2008). Combining TMS and EEG to study cognitive function and cortico-cortico interactions. Behav Brain Res, 191(2), 141-147.

Taylor, P. C., Walsh, V., \& Eimer, M. (2010). The neural signature of phosphene perception. Hum Brain Mapp, 31(9), 1408-1417.

Thut, G., Northoff, G., Ives, J. R., Kamitani, Y., Pfennig, A., Kampmann, F., et al. (2003). Effects of single-pulse transcranial magnetic stimulation (TMS) on functional brain activity: a combined event-related TMS and evoked potential study. Clin Neurophysiol, 114(11), 2071-2080.

Thut, G., Veniero, D., Romei, V., Miniussi, C., Schyns, P., \& Gross, J. (2011). Rhythmic TMS causes local entrainment of natural oscillatory signatures. Curr Biol, 21(14), 1176-1185.

Veniero, D., Bortoletto, M., \& Miniussi, C. (2009). TMS-EEG co-registration: on TMS-induced artifact. Clin Neurophysiol, 120(7), 1392-1399.

Virtanen, J., Ruohonen, J., Naatanen, R., \& Ilmoniemi, R. J. (1999). Instrumentation for the measurement of electric brain responses to transcranial magnetic stimulation. Med Biol Eng Comput, 37(3), 322-326. 


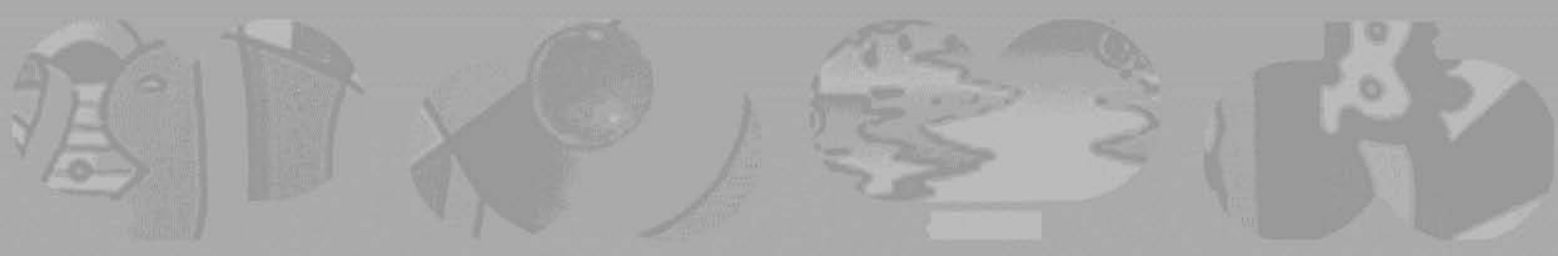

\section{Chapter 6 GeneraI discussion}
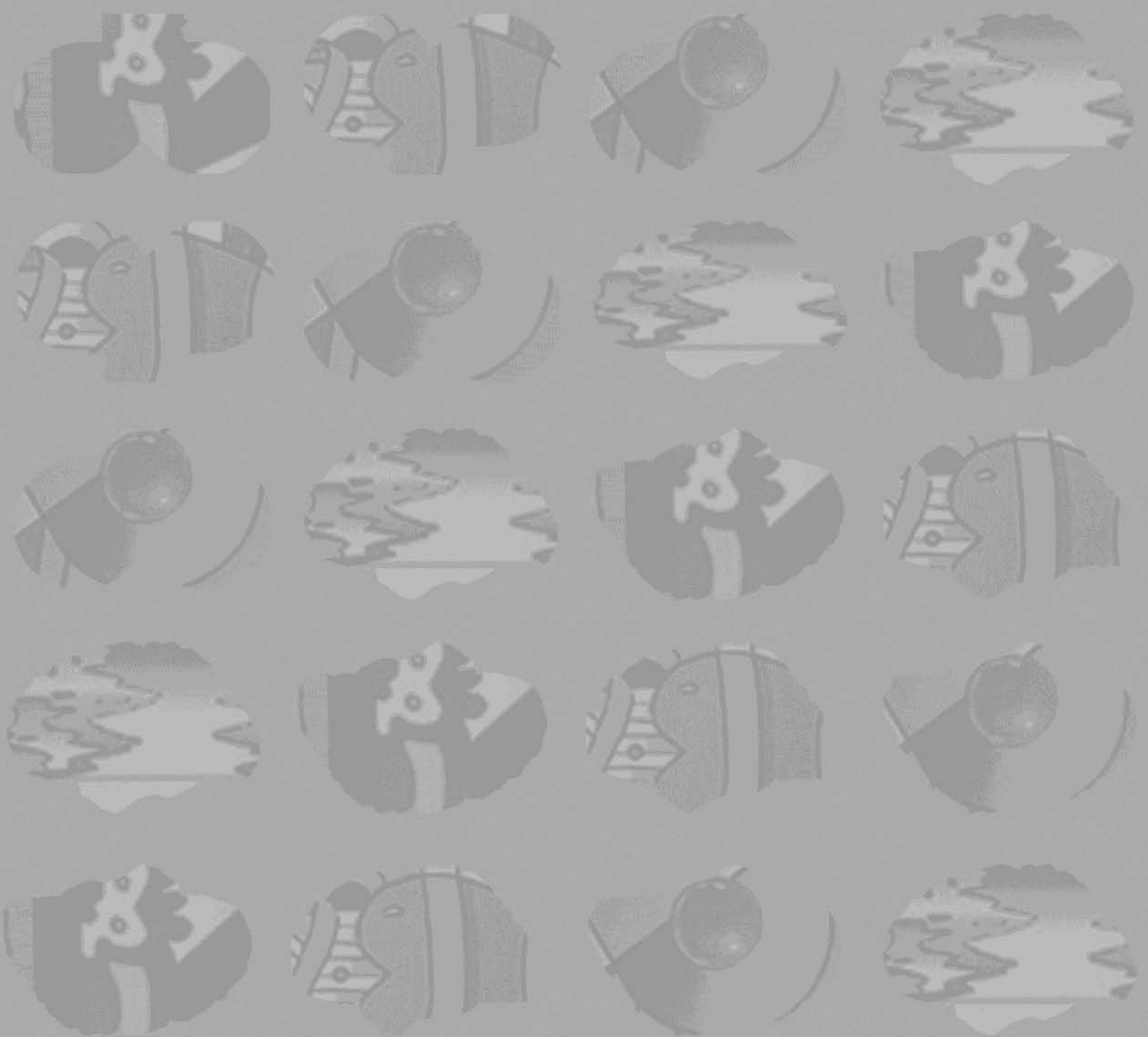
Visual perception is mesmerizing. To all of us who enjoy the blessing of visual experience every day, but also in particular to neuroscientists who are engaged in mapping our many cognitive and perceptual capacities to the human brain. The heavy dependence of the human race on vision, a trait we share with many living creatures, inspired numerous researchers to study human visual perception, and they have taken up the challenge to locate the many aspects of this sensation in the brain. So far, this has rendered a great deal of knowledge on the workings of the human visual system and the brain areas that are involved. The visual pathway running from the eyes, via the thalamus, to the cortical visual system has been traced. Within the cortex, specialized modules dedicated to the processing of single visual features have been identified, and one can thus conclude that the visual hierarchy has been successfully mapped.

At the early stages of this hierarchy however, the visual signal is not parceled out yet, and information about all features is still present in the neural code. Therefore, it makes sense to assume that the function of these cortical areas is one of a more general nature. From the hierarchical perspective on the organization of the visual brain, the relay of information to ascending brain regions has been assigned to early visual cortex (EVC). But lately EVC has been implied in more than the mere passive transfer of information to higher-order brain areas, and the existence of massive feedback connections within the visual system contribute to the idea that visual information processing does not terminate in the extrastriate visual 'modules'. By now, many visual processes have been suggested to be modulated by recurrent processing (Bullier, 2001; Hochstein \& Ahissar, 2002; Lamme \& Roelfsema, 2000; Pollen, 1999), most notably the initiation of visual awareness.

The research conducted within the framework of this thesis aimed at disentangling the role of EVC across the visual domain. Hereto, chronometric transcranial magnetic stimulation (TMS) was employed to momentarily disrupt the functioning of EVC during the execution of a range of visual tasks. To get a grasp on the physiological mechanisms by which TMS can modulate vision, concurrent TMS-EEG data acquisition is required. The suitability of two different TMS systems for simultaneous EEG recording was assessed, and the visual evoked responses (VEPs) in a pre-stimulus TMS masking paradigm were evaluated. In the following sections, the obtained results will be recapitulated and their significance will be evaluated. Furthermore, recommendations for future research will be provided.

\section{Main results}

When perturbing EVC of healthy individuals with single pulses of TMS during execution of a visual discrimination task, out of the broad range of tested time windows none appeared at which performance remained intact, while awareness was suppressed. Post-hoc selection of the trials on the basis of awareness ratings revealed three time window $(-40 \mathrm{~ms}, 80$ and $100 \mathrm{~ms})$ at which discri- 
mination accuracy exceeded chance level for the trials rated as 'unseen'. These results are in line with earlier accounts of TMS-induced blindsight, all of which were brought about by post-stimulus EVC TMS (Boyer, Harrison, \& Ro, 2005; Christensen, Kristiansen, Rowe, \& Nielsen, 2008; Jolij \& Lamme, 2005), although it might rather reflect a difference in sensitivity between the subjective and objective measures, than genuine TMS-induced blindsight.

The classic $80-100 \mathrm{~ms}$ post-stimulus time window of effective masking, which was already reported in the earliest of occipital TMS studies and has been consistently demonstrated ever since, revealed itself here again, both for self-reported visual awareness and discrimination performance. Occipital TMS at none of the later time windows impaired either subjective awareness or task performance.

The most remarkable finding is the pre-stimulus TMS masking effect. When the TMS pulses preceded the visual stimulus by 80 to $40 \mathrm{~ms}$, both self-reported visual awareness as well as discrimination performance dropped significantly. Although pre-stimulus visual suppression with TMS had been discovered before, it had been predominantly attributed to eye blinking (Beckers \& Homberg, 1991; Corthout, Uttl, Juan, Hallett, \& Cowey, 2000; Sack, Kohler, Linden, Goebel, \& Muckli, 2006). However, post hoc sorting of trials and subsequent analyses of eye blink free trials in isolation demonstrated residual significant pre-stimulus decreases of both awareness and performance. In contrast, placebo (sham) and control site (vertex) TMS did not reveal any pre-stimulus TMS effect. Together, the included control conditions ruled out eye blinking, and other non-neural TMS effects, like aversive scalp sensation and clicking sounds, as the causes of pre-stimulus TMS masking and thus falsified interpretations that dismiss the effect as a non-neural TMS confound.

In a subsequent study, the same participant sample performed a priming task. The experimental set-up was completely identical, apart from the task instructions. Participants were now to report the orientation of the second stimulus, which they were previously instructed to ignore. The priming effect, defined as the difference in reaction times between incongruent and congruent trials, was reduced when EVC was stimulated by means of TMS $60-100 \mathrm{~ms}$ postprime. The conclusion of Sack et al., (2009) that behavioral priming relies on an intact geniculostriate pathway, was thus corroborated by these results. Moreover, pre-stimulus occipital TMS negatively affected the behavioral influence of the prime. Interestingly, this conclusion could only be drawn after comparing the experimental results with the results of both control conditions: placebo and control site TMS. The non-specific effects of pre-stimulus TMS apparently cause increased priming and this baseline increase cancels out the depressant neural effect of TMS.

Finally, functional relevance of EVC for visual short-term memory (VSTM) was established. EVC was again stimulated by single pulses of TMS at multiple different time points, but this time during execution of a modified change detection task. The detrimental effect of EVC TMS on short-term memory consolidation proved 1) spatially specific, because only contralateral TMS affected 
memory performance, 2) temporally specific, because only TMS delivered 200 $\mathrm{ms}$ in the retention interval affected memory performance, and 3) load-dependent, because only trials in which the memory load was high were affected. An additional visual memory masking experiment used the exact same paradigm, except for the type of memory mask, which now consisted of a visual mask instead of a TMS pulse, and confirmed the temporal and spatial specificity, and load-dependence of the TMS results.

\section{Early visual cortex: relay station or recurrently relevant?}

All visual processes under study have been shown to depend on EVC. Even though this might not appear to be a revelation, this finding is informative because of the existence of an alternative subcortical route from optical tract to higher-level (visual) cortex. Supposedly, unconscious visual input reaches extrastriate areas through this pathway in blindsight patients (Goebel, Muckli, Zanella, Singer, \& Stoerig, 2001). If vision in healthy individuals were to function in the same manner, then visual discrimination and priming did not necessarily have to suffer from temporary EVC interference. However, the current results indicate otherwise.

There are two possible explanations for these findings. First, the permanent damage to primary visual cortex (V1) in blindsight might not be comparable to the temporal 'virtual lesion' induced with TMS. The plasticity of the human brain has been demonstrated on many occasions, and in the case of V1 trauma, the visual system might adapt to the new situation by an increased dependence on processing through the subcortical route. Second, it is important to note that, even if behavioral measures were negatively affected by EVC TMS, neither visual awareness, discrimination nor priming was ever fully obliterated. There are various likely causes for the residual performance observed here. Inadequate fixation by participants, for example, would cause TMS suppression of irrelevant visual field locations. Nevertheless, there is the possibility that part of the stimulus-related signal reaches higher-level visual areas via a processing pathway that circumvents $\mathrm{EVC}$, and that performance is therefore not altogether abolished. A recent study has rendered further support for the conclusion. When the conscious awareness of a prime stimulus was completely abolished by a visual mask stimulus, as in a classical masked priming paradigm, EVC TMS proved to also interfere with priming (Koivisto, Henriksson, Revonsuo, \& Railo, 2012).

Given the fact that EVC is involved in a broad range of visual processes, the next question in need of an answer concerns the functionality of EVC. The temporal patterns of visual awareness, discrimination and behavioral priming are remarkably similar, all three showing EVC involvement in a pre-stimulus time period revolving around $-60 \mathrm{~ms}$ and a post-stimulus time period revolving around $100 \mathrm{~ms}$. Regardless of whether this post-stimulus time window reflects feedforward or feedback activity, under the assumption that recurrent connec- 
tivity constitutes visual awareness, one would at least expect to see two (poststimulus) time windows of EVC involvement in visual awareness. Here, only one such time window was identified, and the results therefore do not support theories implying recurrent processing in visual awareness. At the same time, it would be too early to write off models of visual awareness that involve recurrent processing, only on the basis of these results.

A recent model of recurrent processing has been put forward that attributes visual awareness to local recurrent processing and figure-ground segregation to global recurrent processing (Koivisto, Railo, Revonsuo, Vanni, \& SalminenVaparanta, 2011; Koivisto, Railo, \& Salminen-Vaparanta, 2011). If recurrent processing within visual cortex establishes conscious perception, then the timescale of EVC's sequential involvement in feedforward and feedback processing might be very small (Boehler, Schoenfeld, Heinze, \& Hopf, 2008; Fahrenfort, Scholte, \& Lamme, 2007). Possibly, the current chronometric design did not have sufficient temporal resolution to pick up on the temporal separation between feedforward and feedback processing. The first part of the broad poststimulus TMS masking interval would then correspond to the feedforward sweep and the second part would correspond to feedback processing. Still, it remains conceivable that feedforward and feedback activity within EVC are inherently not dissociable with TMS. If feedback commences the instant the first feedforward signals arrive, stable local loops of recurrent activity are instantiated, and the two forms of processing continuously interact. In these circumstances, no discrete time points of feedforward and feedback activity are to be detected, and increasing the temporal resolution of the TMS design will not be an effective improvement in experimental design. TMS alone is not a suitable method to detect such an interwoven version of recurrent processing, because it does not allow any conclusion about activity at the neuronal level.

Summarizing, EVC is functionally relevant for visual perception, whether the processed visual information is utilized to create a phenomenal, conscious experience, to pass judgment on the nature of the visual percept, or to prime the cortical system towards a future response. The time window of EVC relevance for all these processes is $\sim 90 \mathrm{~ms}$ post-stimulus. Theories assigning visual awareness to recurrent processing cannot be supported based on the current data, because a second post-stimulus time window of EVC relevance for visual awareness is lacking.

Although not specific to visual awareness, the here presented data demonstrate a later ( $>90 \mathrm{~ms}$ ) time window at which EVC processing contributes to task performance. Visual short-term memory is affected by occipital TMS when applied $200 \mathrm{~ms}$ into the retention interval. Obviously, at this time the initial perceptual stage of visual processing has ceased, and the TMS mask interacts with a different kind of visual process, namely memory consolidation. The occipital TMS pulse probably interferes with the neural representation of the visual memory trace, an interesting finding because fMRI studies have challenged the involvement of EVC in visual short-term memory (Courtney, 
Ungerleider, Keil, \& Haxby, 1997; Munk, et al., 2002; Tambini, Ketz, \& Davachi, 2010).

Moreover, the TMS pulse does not have this effect at any given time window, but only specifically when delivered $200 \mathrm{~ms}$ after stimulus offset. The temporal specificity of the TMS memory masking effect implies that short-term memory consolidation is a dynamic process, rather than a matter of a stable visual representation constructed during the encoding phase and retained in EVC over time, until action is taken upon it, or until the signal decays. Recurrent processing comes back into play here. As the pulse arrives too late to interfere with feedforward processing, occipital TMS 200ms post-stimulus offset must interfere with a feedback loop from higher-level (visual) brain areas back to EVC. It has been proposed that recurrent processing occurs as a way to enable the system to continuously compare the information processed in extrastriate cortex to the current visual representation in EVC (Di Lollo, Enns, \& Rensink, 2000). A visual memory mask or an occipital TMS pulse alters the visual representation in EVC such that it conflicts with the memory representation being processed further along the visual hierarchy, leading to a decrease in memory performance.

In conclusion, EVC appears to be more than merely a relay station of visual information: EVC plays a role in visual short-term memory beyond the encoding of the visual input into the retention of the visual representation. Recurrent processing sweeps probably are the basis for this longer-lasting EVC involvement.

\section{Pre-stimulus TMS masking}

Pre-stimulus visual suppression is much less established than the classic $\sim 90$ ms time window of TMS masking, let alone that there is any consensus about the underlying neural mechanism. Until now, many researchers believed pre-stimulus TMS masking to be caused by eye blinking, or other non-neural TMS effects (Beckers \& Homberg, 1991; Corthout, et al., 2000; Sack, et al., 2006). The few authors, who considered their pre-stimulus findings of neural origin, gave various interpretations as to what that origin may be (Cowey, 2008; Laycock, Crewther, Fitzgerald, \& Crewther, 2007; Stevens, McGraw, Ledgeway, \& Schluppeck, 2009). The current results exclude non-neural TMS effects as the cause of pre-stimulus masking, but the underlying neural processes remain elusive. The combined empirical evidence from a number of recent studies on TMS-induced phosphene perception (Dugue, Marque, \& VanRullen, 2011; Romei, et al., 2008) and neural correlates of visual perception (Busch, Dubois, \& VanRullen, 2009; Mathewson, Gratton, Fabiani, Beck, \& Ro, 2009; Romei, Gross, \& Thut, 2010; Thut, Nietzel, Brandt, \& Pascual-Leone, 2006) points in the direction of TMS-induced alpha oscillations, which in turn make EVC unreceptive to subsequent visual input. 
The neural mechanism underlying the post-stimulus TMS masking effect has been suggested to be the direct suppression of the stimulus-related signal within EVC rather than an increase in noise (Harris, Clifford, \& Miniussi, 2008). This idea cannot be transferred to pre-stimulus occipital TMS as there is no signal to meddle with at the moment of EVC TMS. So, there must be an alternative way in which TMS can influence (later) stimulus processing. Possibly, the application of TMS brings EVC in a certain 'state', in which it lingers until the stimulus-related input arrives. The incompatibility of EVC state and stimulus processing demands could then be the reason why stimulus-related behavior is impaired, as revealed by lower performance on a visual discrimination task, for example.

This model relies on two assumptions, each of which have been supported by empirical evidence: 1) TMS can put a brain region in a certain state; and 2) the brain state induced in EVC by occipital TMS is incompatible with stimulus processing. Oscillatory neuronal activity in the alpha frequency range $(\sim 10 \mathrm{~Hz})$ is generally associated with neural inhibition (Klimesch, Sauseng, \& Hanslmayr, 2007), but it has particularly been linked to occipital cortex (Busch, et al., 2009; Toscani, Marzi, Righi, Viggiano, \& Baldassi, 2010). Occipital cortical excitability changes due to TMS interact with alpha power at the moment of pulse delivery (Romei, et al., 2008). A visually masked stimulus is detected less often in the presence of alpha oscillations in occipital cortex prior to stimulus presentation. When pre-stimulus alpha power is high, the phase that the alpha wave is in at the time of stimulus onset matters as well (Mathewson, et al., 2009). Recently, phosphene perception induced by TMS was revealed to correlate with the phase of alpha oscillations in the $400 \mathrm{~ms}$ preceding the TMS pulse (Dugue, et al., 2011), another indication that alpha phase modulates visual perception. Given these recent findings, it is plausible that pre-stimulus TMS might exert its effect by the induction of oscillatory alpha activity in occipital cortex that, in turn, inhibits signal processing. And there is reason to believe that probing occipital cortex with TMS in fact causes rhythmic oscillations of neuronal firing in the alpha frequency range (Rosanova, et al., 2009). Taken together, these findings link TMS to alpha, and alpha to visual suppression. Therefore, we consider TMS-induced rhythmic alpha activity a plausible candidate brain state to bridge the temporal gap between TMS pulse and visual stimulus.

The optimal way to test this idea is via the simultaneous acquisition of neurophysiological data during TMS-induced suppression, because they encompass a direct measure of the behavior of neuronal populations in the cortex. Moreover, the pre- and post-stimulus TMS masking effect have both been found to be highly temporally specific, and the neural mechanism underlying these TMS effects are therefore expected to be of a dynamic nature. Concurrent EEG recording with its excellent temporal resolution is therefore the preferred method when it comes to disentangling the neural underpinnings of pre- and post-stimulus TMS masking.

Whatever the neural mechanism of the pre-stimulus TMS effect will prove to be, the results of the research presented here indicate that it interferes with 
visual perception holistically, because visual awareness, visual discrimination and priming all suffer from pre-stimulus TMS stimulation of EVC.

\section{The Neural Correlates of Subliminal Priming}

The priming effect displayed the identical temporal pattern of EVC dependence as visual awareness and discrimination performance. However, at the behavioral level these processes have been dissociated through the demonstration of subliminal priming. Different accounts on the processing routes of unconscious vision have been suggested. As mentioned before, the idea that unconscious visual information takes a detour via subcortical structures is not in line with our findings. In contrast, theories about recurrent processing and its crucial role in visual awareness would predict that we are not aware of information that is processed by our brains in a strictly feedforward manner. On the basis of the current results, this suggestion seems more plausible. After all, there was only one post-stimulus time period of TMS interference with behavioral priming, and it is conceivable that this represents the feedforward sweep. However, the failure to detect a second, later time window of selective visual awareness suppression casts doubt on this interpretation, because it means that a neural dissociation in EVC, comparable to the behavioral dissociation in psychophysical studies on subliminal priming, was not achieved.

In general, it is unfortunate that subliminal priming has been considered mainly a tool in the search for the neural correlates of consciousness (NCC), which is supposedly captured in the different neural response to supraliminal versus subliminal priming conditions. What is absent during subliminal priming, i.e. the NCC, has been the center of focus and the neural activity coding for the unconscious processing that takes place has been mainly ignored. Analogous to the subtraction logic employed in search of the NCC, one could subtract the neural response to stimuli that evoke a behavioral response from those that do not, and define the Neural Correlates of Subliminal Priming (NCSP) as that particular neural activity that survived this subtraction. Studying the NCSP in this way can lead to important insights as to what neural activity underlies the behavioral influence that environmental stimuli can have.

Proponents of the Global Neuronal Workspace (GNW) model of visual awareness have mainly employed subliminal priming paradigms in their search for the NCC (Dehaene, et al., 2001; Dehaene, et al., 1998; Kouider \& Dehaene, 2009; Kouider, Dehaene, Jobert, \& Le Bihan, 2007; Kouider, Eger, Dolan, \& Henson, 2009). Nonetheless, the GNW model also makes some clear predictions when it comes to the NCSP (Dehaene, Changeux, Naccache, Sackur, \& Sergent, 2006). If a physical stimulus is not strong enough or when focused attention is directed elsewhere, the information is not globally accessible, and the stimulus is thus not consciously perceived. But priming on the basis of this information can still occur, if the bottom-up strength of the prime stimulus is strong enough and it will initiate local neuronal synchrony. Therefore, within the GNW frame- 
work, the strength of information processing restricted to the sensory cortex can be said to be the NCSP. As mentioned before though, to prove the claim that the bottom-up strength of the visual stimulus determines whether a stimulus has any behavioral impact, conditions of successful and unsuccessful priming should be compared, and should result in a higher activation amplitude in extrastriate areas during successful priming. So far, the no-priming condition has not been incorporated in the design of subliminal priming experiments and this crucial comparison therefore has not been performed.

\section{Methodological suggestions}

The here presented studies supply interesting new insights concerning EVC functioning. In addition, they also carry a number of methodological implications that are relevant to TMS research in general. The results from Chapter 3 emphasize the significance of selecting appropriate control conditions in order to successfully isolate the process that is under investigation. There are two major points that deserve particular interest.

First, the attentional demands of the task interact with the non-specific arousing or attention-drawing effects of TMS. The behavioral influence of the prime stimulus increased when TMS was applied over a non-relevant control site (vertex) prior to stimulus onset, but no increase in accuracy during visual discrimination was observed ceteris paribus. A plausible explanation for this discrepancy is the difference in top-down attention in the two situations. During stimulus discrimination, attention is already directed at the first, to-bediscriminated stimulus. On the contrary, during priming participants are required to discriminate a second stimulus, and their attention is most likely directed towards this target stimulus instead of the prime. In this case, the bottom-up attention grasped by the TMS pulse just before prime-onset can be beneficial to prime perception, and consequently to the behavioral influence the prime exerts.

Second, placebo (sham) TMS and control site TMS are not equally suitable to control for the non-neural side effects of TMS. Pre-stimulus vertex TMS revealed an increase in behavioral priming, whereas sham TMS did not. There are obvious differences between the two control measures. The absence of a real magnetic field stimulating the scalp makes sham TMS much more agreeable than real or control site TMS, and sham TMS therefore only serves as a control for the clicking sound of TMS. The obvious conclusion would be to always opt for control site TMS instead of sham TMS. However, it might not be easy to identify a control site that is irrelevant to task execution. These problems easily arise with complex task, or with tasks for which the brain shows a high degree of lateralization. Finding a superfluous brain area within the same hemisphere might prove difficult. Moreover, to optimally control for the arousing or multisensory integrative effects of TMS, such a control site would need to be similar in laterality to the experimental stimulation site. 
In general, the two points mentioned above emphasize the importance of selecting appropriate control conditions in TMS research. The impact of the control data for the studies presented in this thesis is enormous. Without these, the main conclusion about pre-stimulus EVC involvement in behavioral priming would have been completely reversed. In particular, these findings suggest that timing of the TMS pulse, attentional demands of the task and the experimental stimulation site, all need to be considered, because the non-specific effects of TMS appear to depend on these variables, and therefore, so should the control conditions.

Concerning the concurrent combination of TMS and EEG, we preferred the Magstim over the Magpro set-up, because the return to baseline of the EEG signal after TMS pulse release proved much prolonged with the latter system. The Magpro system has the advantage that stimulator recharge can be delayed and the EEG signal is thus not further contaminated by any recharge artifacts, but this did not outweigh the disadvantage of the prolonged post-pulse recovery. However, combined TMS-EEG studies have been successfully executed with this TMS system (Reichenbach, Whittingstall, \& Thielscher, 2011).

Regarding EEG data analysis, we found that with our set-up the TMS template subtraction approach worked properly and rendered a cleaned EEG signal within the range of normal VEPs, even if the earliest component (C1) was not recovered. This means that modulations of the EEG signal across experimental conditions are at least no longer masked by the high amplitudes of the TMS-induced artifacts. Further cleaning of the EEG data by rejection of artifactual independent components mainly improved the signal within $50 \mathrm{~ms}$ after the TMS pulse. Here, this time interval was part of the pre-stimulus domain and thus not relevant to the generated VEPs. However, research on VEPs in poststimulus TMS masking paradigms might benefit from additional signal cleaning through independent component analysis (ICA), because restricting the TMSaffected EEG data window is even more crucial then. Furthermore, only a single ICA algorithm was tested here and rejected components were selected manually. Perhaps other ICA algorithms or automatic component rejection might prove more beneficial to the purpose of cleaning TMS-distorted EEG data.

\section{Future research}

The research within this thesis has revealed that pre-stimulus TMS masking has a neural basis. The logical next question to ask is what the neural basis of the pre-stimulus TMS masking effect exactly is, and how it differs from poststimulus TMS masking. Simultaneously combining TMS with EEG is the preferred method of investigation to study this question. As mentioned in Chapter 5, we plan to link the EEG data acquired in the context of that chapter to behavioral responses. Post-hoc classification of trials on the basis of selfreported visual awareness or accuracy, and contrasting the EEG responses to successfully versus unsuccessfully masked stimuli is one approach. Next, in- 
spection of the pre-TMS time period is relevant, because it could give insight as to why a TMS pulse suppresses visual perception on some trials, while on other trials, it does not.

Another open question concerns the function of recurrent processing. Feedback connections have been implied in many visual processes so far, but still it is not exactly clear what their role in visual perception exactly entails. This is especially unclear for visual awareness. Here, we did not find temporally separated time windows of awareness suppression by TMS. Nevertheless, these results are still reconcilable with particular versions of recurrent processing theory. Future research should aim to scrutinize the idea that local recurrent processing underlies visual awareness.

So far, many priming studies have focused on the comparison between supra- and subliminal priming, and what it can tell about the neural correlates of visual awareness. This is unfortunate, because studying subliminal priming to learn about the NCSP can lead to important new insights about the neural activity underlying the behavioral influence that environmental stimuli exert, or, in the case of unsuccessful priming, do not exert. Future studies might want to look into this, as yet, unexplored field of study.

Finally, on the basis of the control data presented in Chapter 3, we propose that the attentional demands of a task might be of relevance when selecting appropriate control conditions in TMS paradigms. A methodological study examining the interaction between attentional task demands and auditory or somatosensory side effects of TMS in order to investigate this claim would be of interest of the TMS community in general. As would a study, that systematically compares sham TMS versus control site TMS on their suitability to control for particular non-specific TMS effects across different cognitive or perceptual tasks. 


\section{REFERENCES}

Beckers, G., \& Homberg, V. (1991). Impairment of visual perception and visual short term memory scanning by transcranial magnetic stimulation of occipital cortex. Exp Brain Res, 87(2), 421432.

Boehler, C. N., Schoenfeld, M. A., Heinze, H. J., \& Hopf, J. M. (2008). Rapid recurrent processing gates awareness in primary visual cortex. Proc Natl Acad Sci U S A, 105(25), 8742-8747.

Boyer, J. L., Harrison, S., \& Ro, T. (2005). Unconscious processing of orientation and color without primary visual cortex. Proc Natl Acad Sci U S A, 102(46), 16875-16879.

Bullier, J. (2001). Feedback connections and conscious vision. Trends Cogn Sci, 5(9), 369-370.

Busch, N. A., Dubois, J., \& VanRullen, R. (2009). The phase of ongoing EEG oscillations predicts visual perception. J Neurosci., 29(24), 7869-7876.

Christensen, M. S., Kristiansen, L., Rowe, J. B., \& Nielsen, J. B. (2008). Action-blindsight in healthy subjects after transcranial magnetic stimulation. Proc Natl Acad Sci U S A, 105(4), 1353-1357.

Corthout, E., Uttl, B., Juan, C. H., Hallett, M., \& Cowey, A. (2000). Suppression of vision by transcranial magnetic stimulation: a third mechanism. Neuroreport, 11(11), 2345-2349.

Courtney, S. M., Ungerleider, L. G., Keil, K., \& Haxby, J. V. (1997). Transient and sustained activity in a distributed neural system for human working memory. Nature, 386(6625), 608-611.

Cowey, A. (2008). TMS and visual awareness. In E. Wasserman (Ed.), Oxford handbook of transcranial stimulation. Oxford: Oxford University Press.

Dehaene, S., Changeux, J. P., Naccache, L., Sackur, J., \& Sergent, C. (2006). Conscious, preconscious, and subliminal processing: a testable taxonomy. Trends Cogn Sci, 10(5), 204-211.

Dehaene, S., Naccache, L., Cohen, L., Bihan, D. L., Mangin, J. F., Poline, J. B., et al. (2001). Cerebral mechanisms of word masking and unconscious repetition priming. Nat Neurosci, 4(7), 752-758.

Dehaene, S., Naccache, L., Le Clec, H. G., Koechlin, E., Mueller, M., Dehaene-Lambertz, G., et al. (1998). Imaging unconscious semantic priming. Nature, 395(6702), 597-600.

Di Lollo, V., Enns, J. T., \& Rensink, R. A. (2000). Competition for consciousness among visual events: the psychophysics of reentrant visual processes. J Exp Psychol Gen, 129(4), 481-507.

Dugue, L., Marque, P., \& VanRullen, R. (2011). The phase of ongoing oscillations mediates the causal relation between brain excitation and visual perception. J Neurosci, 31(33), 11889-11893.

Fahrenfort, J. J., Scholte, H. S., \& Lamme, V. A. (2007). Masking disrupts reentrant processing in human visual cortex. J Cogn Neurosci, 19(9), 1488-1497.

Goebel, R., Muckli, L., Zanella, F. E., Singer, W., \& Stoerig, P. (2001). Sustained extrastriate cortical activation without visual awareness revealed by fMRI studies of hemianopic patients. Vision Res, 41(10-11), 1459-1474.

Harris, J. A., Clifford, C. W., \& Miniussi, C. (2008). The functional effect of transcranial magnetic stimulation: signal suppression or neural noise generation? J Cogn Neurosci, 20(4), 734-740.

Hochstein, S., \& Ahissar, M. (2002). View from the top: hierarchies and reverse hierarchies in the visual system. Neuron, 36(5), 791-804.

Jolij, J., \& Lamme, V. A. (2005). Repression of unconscious information by conscious processing: evidence from affective blindsight induced by transcranial magnetic stimulation. Proc Natl Acad Sci US A, 102(30), 10747-10751.

Klimesch, W., Sauseng, P., \& Hanslmayr, S. (2007). EEG alpha oscillations: the inhibition-timing hypothesis. Brain Res Rev., 53(1), 63-88.

Koivisto, M., Henriksson, L., Revonsuo, A., \& Railo, H. (2012). Unconscious response priming by shape depends on geniculostriate visual projection. EurJ Neurosci, 35[4], 623-633.

Koivisto, M., Railo, H., Revonsuo, A., Vanni, S., \& Salminen-Vaparanta, N. (2011). Recurrent processing in V1/V2 contributes to categorization of natural scenes. J Neurosci, 31(7), 24882492.

Koivisto, M., Railo, H., \& Salminen-Vaparanta, N. (2011). Transcranial magnetic stimulation of early visual cortex interferes with subjective visual awareness and objective forced-choice performance. Conscious Cogn, 20(2), 288-298.

Kouider, S., \& Dehaene, S. (2009). Subliminal number priming within and across the visual and auditory modalities. Exp Psychol., 56(6), 418-433.

Kouider, S., Dehaene, S., Jobert, A., \& Le Bihan, D. (2007). Cerebral bases of subliminal and supraliminal priming during reading. Cereb Cortex, 17(9), 2019-2029. 
Kouider, S., Eger, E., Dolan, R., \& Henson, R. N. (2009). Activity in face-responsive brain regions is modulated by invisible, attended faces: evidence from masked priming. Cereb Cortex, 19(1), 1323.

Lamme, V. A., \& Roelfsema, P. R. (2000). The distinct modes of vision offered by feedforward and recurrent processing. Trends Neurosci, 23(11), 571-579.

Laycock, R., Crewther, D. P., Fitzgerald, P. B., \& Crewther, S. G. (2007). Evidence for fast signals and later processing in human V1/V2 and V5/MT+: A TMS study of motion perception. J Neurophysiol, 98(3), 1253-1262.

Mathewson, K. E., Gratton, G., Fabiani, M., Beck, D. M., \& Ro, T. (2009). To see or not to see: prestimulus alpha phase predicts visual awareness. J Neurosci, 29(9), 2725-2732.

Munk, M. H., Linden, D. E., Muckli, L., Lanfermann, H., Zanella, F. E., Singer, W., et al. (2002). Distributed cortical systems in visual short-term memory revealed by event-related functional magnetic resonance imaging. Cereb Cortex, 12(8), 866-876.

Pollen, D. A. (1999). On the neural correlates of visual perception. Cereb Cortex, 9(1), 4-19.

Reichenbach, A., Whittingstall, K., \& Thielscher, A. (2011). Effects of transcranial magnetic stimulation on visual evoked potentials in a visual suppression task. Neuroimage, 54(2), 13751384.

Romei, V., Brodbeck, V., Michel, C., Amedi, A., Pascual-Leone, A., \& Thut, G. (2008). Spontaneous fluctuations in posterior alpha-band EEG activity reflect variability in excitability of human visual areas. Cereb Cortex, 18(9), 2010-2018.

Romei, V., Gross, J., \& Thut, G. (2010). On the role of prestimulus alpha rhythms over occipitoparietal areas in visual input regulation: correlation or causation? J Neurosci, 30(25), 86928697.

Rosanova, M., Casali, A., Bellina, V., Resta, F., Mariotti, M., \& Massimini, M. (2009). Natural frequencies of human corticothalamic circuits. J Neurosci, 29(24), 7679-7685.

Sack, A. T., Kohler, A., Linden, D. E., Goebel, R., \& Muckli, L. (2006). The temporal characteristics of motion processing in hMT/V5+: combining fMRI and neuronavigated TMS. Neuroimage, 29(4), 1326-1335.

Sack, A. T., van der Mark, S., Schuhmann, T., Schwarzbach, J., \& Goebel, R. (2009). Symbolic action priming relies on intact neural transmission along the retino-geniculo-striate pathway. Neuroimage, 44(1), 284-293.

Stevens, L. K., McGraw, P. V., Ledgeway, T., \& Schluppeck, D. (2009). Temporal characteristics of global motion processing revealed by transcranial magnetic stimulation. Eur J Neurosci, 30(12), 2415-2426.

Tambini, A., Ketz, N., \& Davachi, L. (2010). Enhanced brain correlations during rest are related to memory for recent experiences. Neuron, 65(2), 280-290.

Thut, G., Nietzel, A., Brandt, S. A., \& Pascual-Leone, A. (2006). Alpha-band electroencephalographic activity over occipital cortex indexes visuospatial attention bias and predicts visual target detection. J Neurosci, 26(37), 9494-9502.

Thut, G., Veniero, D., Romei, V., Miniussi, C., Schyns, P., \& Gross, J. (2011). Rhythmic TMS causes local entrainment of natural oscillatory signatures. Curr Biol, 21(14), 1176-1185.

Toscani, M., Marzi, T., Righi, S., Viggiano, M. P., \& Baldassi, S. (2010). Alpha waves: a neural signature of visual suppression. Exp Brain Res, 207[3-4], 213-219. 



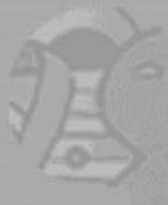
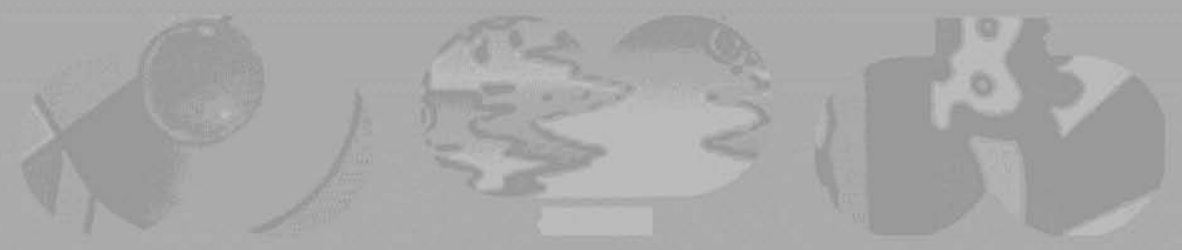

Summary
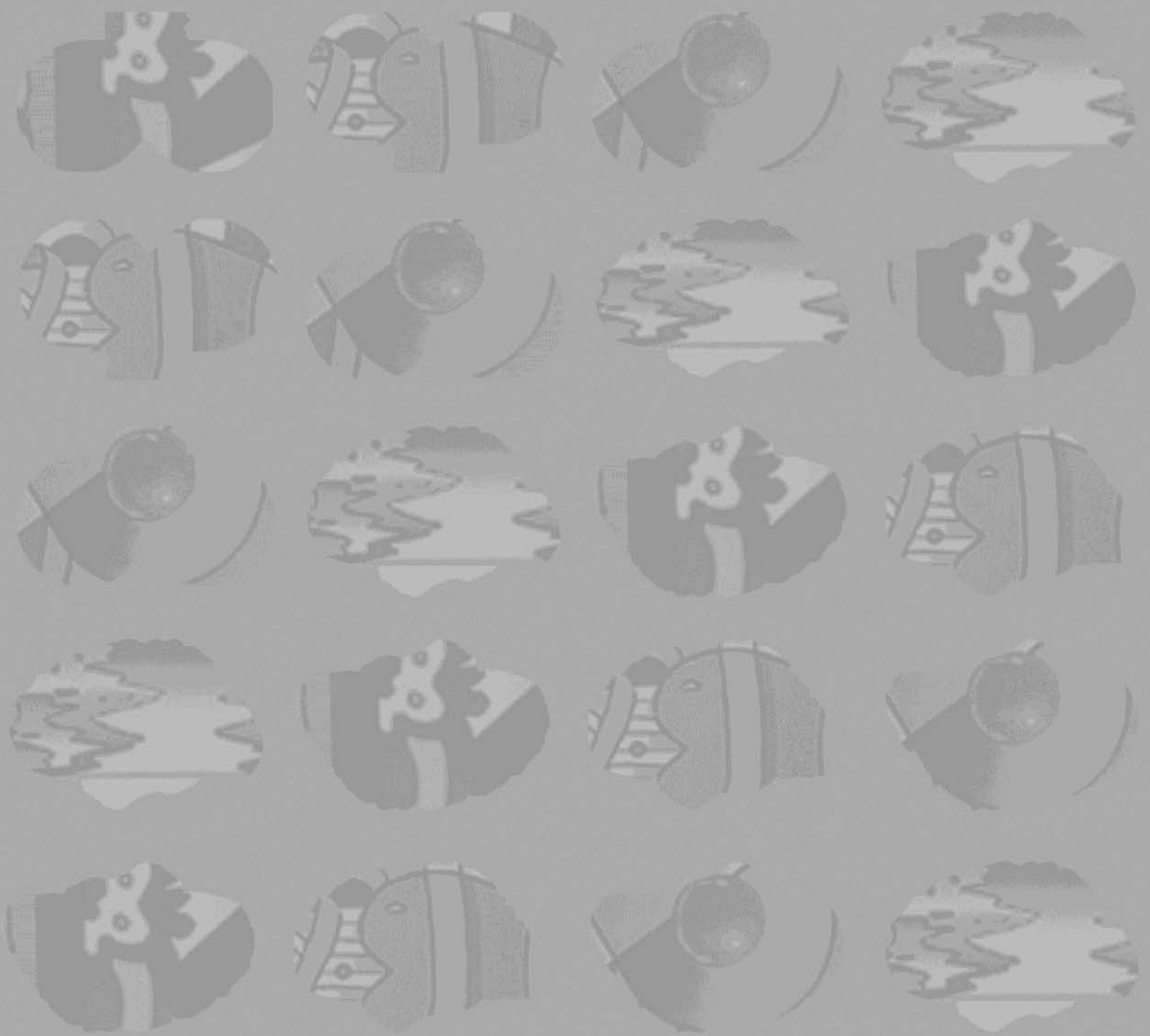
The research presented within this thesis aimed to investigate the functional involvement of early visual cortex (EVC) in a variety of visual processes.

Humans rely heavily on their sense of sight. Knowledge about the way visual information is processed could be benificial to those who suffer from perceptual disorders. Psychophysics, the study of the relation between physical stimulus properties and perception or behavior, has revealed that visual perception can be subdivided in a number of processes. The different visual processes investigated within this thesis are visual awareness, visual discrimination, behavioral priming (based on visual input) and visual shortterm memory. Psychophysical studies have shown that these visual processes are dissociable, which suggests that there are differential neural processes at play as well.

For a number of decades, neuroscience has tried to map the human brain system responsible for the processing of visual information: the visual (neo)cortex. This has led to the discovery that the human visual cortex is organized hierarchically, with increasingly more specialized units processing the visual input in a segmented fashion. Focus has recently shifted from the classical hierarchical view of visual information processing towards the idea that backprojections running from higher-level visual cortex back to the early visual areas have important contributions to visual perception as well. By means of these feedforward-feedback loops of information processing EVC might prove to be more than a simple relay station that passes its input to more sophisticated processing units.

Transcranial magnetic stimulation (TMS) is a brain research method that allows for the temporally and spatially specific manipulation of cortical brain activity through electromagnetic pulses, and it is thus a very suitable method to investigate when activity in EVC is necessary for the execution of a given task or the generation of a given percept. The studies described within this thesis have all employed chronometric single-pulse TMS to examine the functional relevance of EVC for visual awareness, visual discrimination, behavioral priming and visual short-term memory.

The first study (Chapter 2) focused on the role EVC plays in visual awareness and visual discrimination. TMS was applied over EVC at multiple time points relative to the onset of a briefly presented horizontal arrow stimulus. Placebo TMS and TMS over a non-relevant control site were incorporated in the design to control for non-neural side effects of TMS caused by the auditory and somatosensory sensations associated with TMS. In addition, electrooculography (EoG) was recorded to track vertical eye movements. In this way, trials could be sorted post hoc based on the occurrence of eye blinks around the time of stimulus presentation. The study revealed 1) that visual awareness and visual discrimination rely on an intact EVC at overlapping time points and, 2) that EVC is functionally relevant both before and after the presentation of the visual stimulus. The absence of a second post-stimulus TMS effect selective for 
visual awareness does not speak in favor of theories that assign an essential role in visual awareness to feedback loops. The finding of a pre-stimulus TMS effect for both visual awareness and discrimination was present even after the removal of trials containing eye blinks. Moreover, it was absent in the control TMS conditions and it thus appears to be of neural origin. TMS-evoked cortical alpha wave $(\sim 10 \mathrm{~Hz})$ activity might be the neural mechanism underlying this pre-stimulus TMS effect on visual perception.

In Chapter 3, these findings were compared to results acquired with a behavioral priming paradigm. Stimulus and TMS parameters were kept completely identical. However, task instructions changed and participants were now requested to identify the direction of a second arrow stimulus (target) on each trial. The influence of the direction of the first arrow stimulus (prime) on participants' response speed, i.e. the priming effect, was taken as a measure of the behavioral impact of the prime. A post-stimulus TMS pulse time-locked to prime-onset reduced the behavioral impact of the visual stimulus. The time window of this TMS effect overlapped with the post-stimulus effective time windows reported in Chapter 2. In other words, EVC was proven to be functionally relevant to three basic visual processes: visual awareness, discrimination and priming. After correcting for non-neural TMS effects through comparison with the control site TMS data, this appeared also to be the case for the pre-stimulus domain. The placebo TMS data showed a different pattern of results than the control site data. Moreover, the non-neural effects on performance also differed per task, raising the methodological issue about which control for non-neural TMS effects is preferred in which context.

The next project (Chapter 4), investigated the role of early visual areas in a higher-level cognitive task, namely short-term memory. Single pulses of TMS were delivered to EVC at different timings into the retention interval of a visual short-term memory task. In the high memory load condition, a decrease in task accuracy was observed when the TMS pulse followed the offset of the to-beremembered visual display with 200ms. Furthermore, this 'memory masking' effect was selectively demonstrated for TMS pulses applied to the hemisphere contralateral to the visual field of stimulus presentation. Corresponding results were obtained in an additional experiment in which a visual distractor stimulus, rather than a TMS pulse, served as the memory mask. Together these findings indicate that an undisturbed EVC is required during short-term memory consolidation in a temporally and spatially specific manner. As the pulse arrives too late to interfere with feedforward processing, occipital TMS $200 \mathrm{~ms}$ post-stimulus offset must interfere with a feedback loop from higherlevel (visual) brain areas back to EVC. Recurrent processing possibly occurs as a way to enable the system to continuously compare the information processed in extrastriate cortex to the current visual representation in EVC

The findings presented in Chapter 2 to 4 demonstrate that EVC is functionally relevant for multiple visual processes in a time-specific manner both in the pre- and post-stimulus domain. The expected neural mechanisms of action of pre- and post-stimulus TMS over EVC are different, and knowledge of these 
mechanisms can be informative about the exact processes occurring in EVC during visual perception. To gain this knowledge TMS needs to be concurrently combined with neuroimaging methods. Because of the high temporal specificity of the TMS effects under investigation, the simultaneous acquisition of electroencephalography (EEG), a technique with a very high temporal resolution, appears most promising. However, the acquisition of EEG data during TMS proves technically challenging. As described in Chapter 5, after the selection of the most optimal TMS-EEG set-up that could be achieved in the Maastricht Brain Imaging Center, EEG data were collected in a TMS masking paradigm. Under conditions of pre-stimulus TMS, the Visual Evoked Potentials (VEPs) in response to the visual stimulus were completely hidden by the TMS-induced artifact. Nonetheless, after the subtraction of the averaged EEG response to a TMS pulse in isolation from the experimental data, VEPs within the normal VEP amplitude range and similar to those evoked on No TMS trials could be achieved. Future analyses will focus on the comparison of these VEPs to masked versus unmasked visual stimuli for both the pre- and post-stimulus effective TMS time windows.

Other suggestions for future research have been proposed in this thesis. The question whether or not recurrent connectivity is critical in the establishment of visual awareness remains unanswered, and thus requires further research. In addition, the neural signature underlying the behavioral influence of sensory stimuli, the Neural Correlate of Subliminal Priming (NCSP), deserves more scientific attention than it currently receives. Finally, from a TMS methodology perspective, it is essential that the highly relevant issue of what makes an optimal control for non-specific effects in TMS research becomes resolved. 

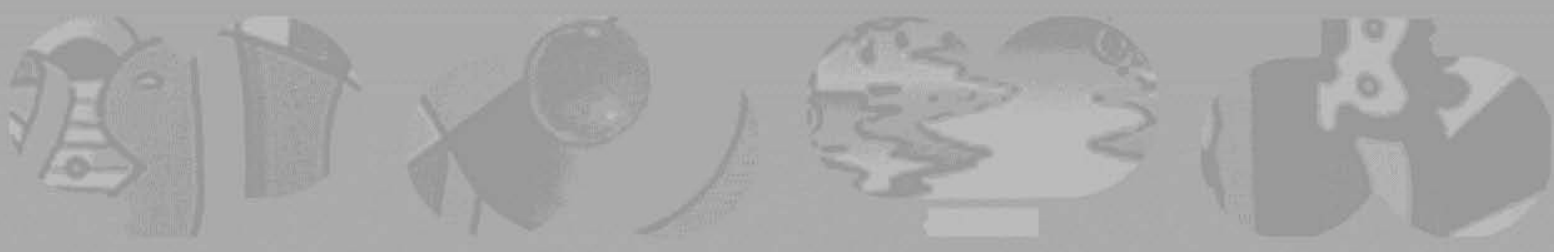

\section{Samenvatting}
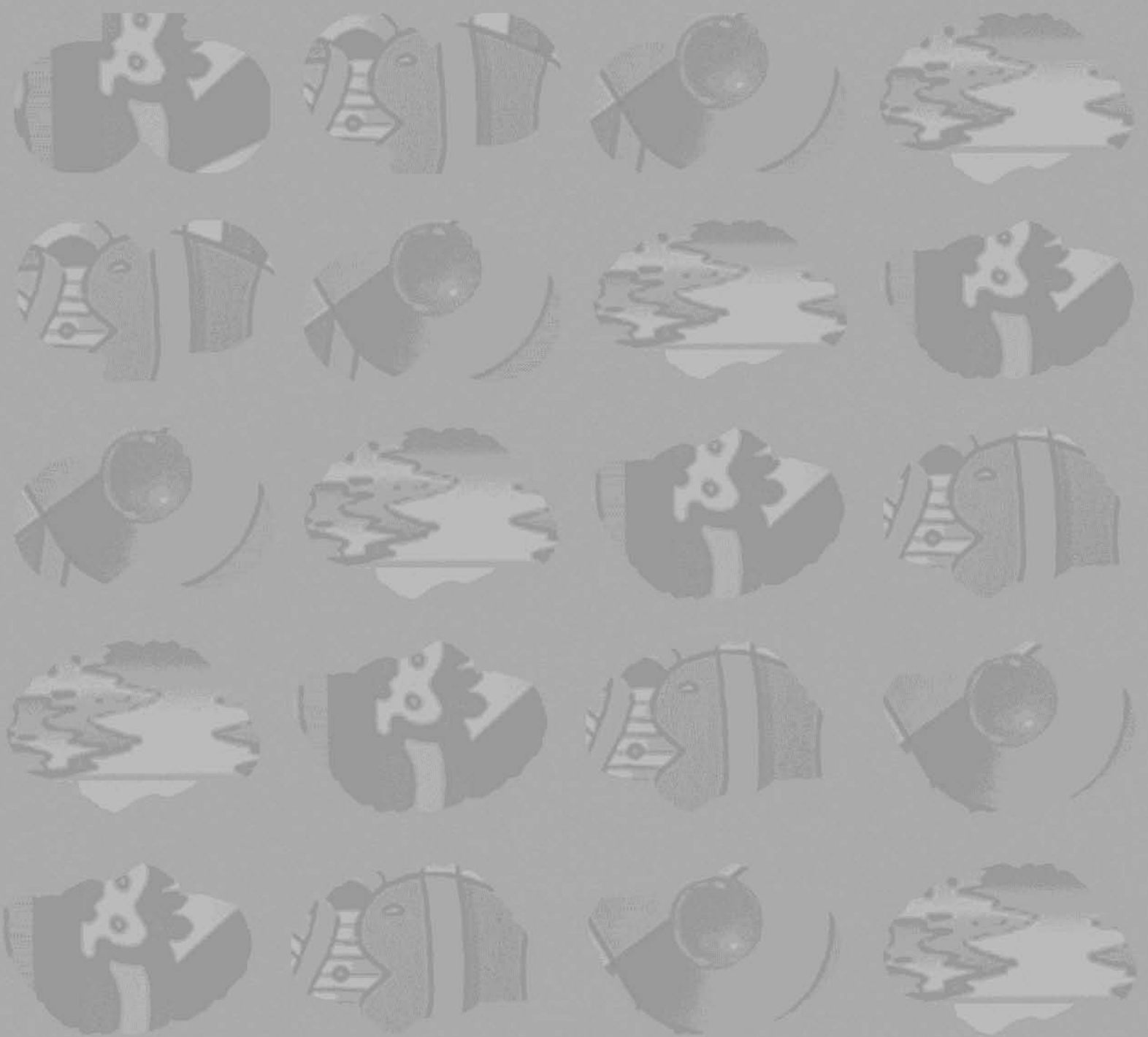
Het onderzoek uit deze these had als doel om de functionele rol die de vroege visuele cortex (EVC) speelt bij verscheidene visuele processen te onderzoeken.

Voor de mens is het gezichtsvermogen van uitermate groot belang. Kennis over de manier waarop mensen visuele informatie verwerken, kan ten goede komen aan oplossingen voor mensen wier visuele waarneming beperkt is.

Psychofysica, de wetenschap die de relatie tussen fysieke stimuli en waarneming of gedrag onderzoekt, heeft aangetoond dat visuele perceptie onderverdeeld kan worden in een aantal processen. De verschillende visuele processen die in deze these aan bod komen zijn visueel bewustzijn, visuele discriminatie, gedragspriming (op basis van visuele informatie) en het visuele kortetermijngeheugen. Psychofysische studies hebben laten zien dat deze visuele processes onafhankelijk zijn, wat suggereert dat er ook verschillende neurale processes bij actief zijn.

Sinds een aantal decennia probeert de neurowetenschap om het deel van het menselijke brein, dat verantwoordelijk is voor het verwerken van visuele informatie (ook wel visuele hersenschors of (neo)cortex genoemd), in kaart te brengen. Dit heeft geleid tot de ontdekking dat de menselijke visuele cortex hierarchisch georganiseerd is, waarbij steeds meer gespecialiseerde verwerkingseenheden de visuele input in toenemende mate gefragmenteerd verwerken. De laatste jaren heeft de focus zich verlegd van dit klassieke hiërarchische oogpunt naar 'feedforward-feedback' circuits. Dat zijn wederkerige projecties van hogere visuele hersengebieden terug naar de vroege visuele cortex. Mogelijk leveren deze een belangrijke bijdrage aan visuele waarneming. Dit kan betekenen dat de EVC meer is dan een simpel doorgeefstation dat binnenkomende informatie alleen maar verder stuurt naar geavanceerdere verwerkingsunits.

Transcraniale Magnetische Stimulatie (TMS) is een onderzoeksmethode die het tijdelijk en plaatselijk manipuleren van corticale hersenactiviteit toestaat door middel van electromagnetische pulsen. Deze methode is hierdoor uitermate geschikt om te onderzoeken wanneer activiteit in de EVC noodzakelijk is voor het uitvoeren van een bepaalde taak of het tot stand komen van een bepaalde waarneming. In de hier beschreven studies is telkens chronometrische TMS toegepast om de functionele relevantie van de EVC voor visueel bewustzijn, visuele discriminatie, gedragspriming en het visuele kortetermijngeheugen te toetsen.

De eerste studie (Hoofdstuk 2) is gericht op de rol die de EVC speelt in visueel bewustzijn en discriminatie van visuele stimuli. TMS werd toegediend aan de EVC op verschillende tijdstippen ten opzichte van het moment waarop een kortdurende horizontale pijl aan proefpersonen getoond werd. Er werd gecontroleerd voor niet-neurale bijwerkingen van TMS, als gevolg van het geluid en onplezierige gevoel op de hoofdhuid, door de toevoeging van placebo TMS en TMS over een irrelevant (controle) hersengebied. Bovendien werd 
Electrooculografie (EoG) gemeten om verticale oogbewegingen te kunnen traceren. Zo konden de trials na afloop van het experiment gesorteerd worden naar trials waarin de proefpersoon geknipperd had en trials zonder oogknippers. Het onderzoek liet zien 1) dat visueel bewustzijn en visuele discriminatie afhankelijk zijn van een intacte EVC op overlappende tijdstippen en 2) dat de EVC functioneel relevant is zowel voorafgaand aan, als na stimuluspresentatie. Het ontbreken van een tweede, later post-stimulus TMS effect, dat selectief het visuele bewustzijn verstoort, spreekt niet voor theorieën die feedbackprocessen een cruciale rol toedichten bij het ontstaan van visueel bewustzijn.

Het gevonden pre-stimulus TMS effect bleek nog steeds aanwezig te zijn nadat de trials met oogknippers uit de dataset verwijderd waren. Omdat het effect in geen van beide TMS controlecondities aanwezig was, lijkt het hier daadwerkelijk om een neuraal effect te gaan. Mogelijk wekt de toegediende TMS-puls ritmische corticale activiteit in de alfafrequentie $(\sim 10 \mathrm{~Hz})$ op, waardoor de EVC niet langer ontvankelijk is voor nieuwe informatie behorende bij een later gepresenteerde visuele stimulus.

Deze resultaten werden in Hoofdstuk 3 vergeleken met de resultaten verkregen in een gedragspriming experiment. De stimulus en TMS parameters werden volledig constant gehouden, terwijl de taakinstructie veranderde. Deelnemers aan het onderzoek werd verzocht om de richting, waarin een tweede pijlstimulus ('target') wees, aan te geven door op een knop te drukken. De invloed van de richting van de eerste pijl ('prime') op de reactiesnelheid van de deelnemers, ook wel het 'priming effect' genoemd, gold als maat van de gedragsmatige impact van de tweede pijl. Een TMS-puls toegediend aan de EVC na presentatie van de prime, veroorzaakte een reductie van het priming effect. Het moment, waarop de reductie plaastvond, overlapte met de effectieve poststimulus tijdstippen uit Hoofdstuk 2. Met andere woorden, EVC bleek functioneel relevant voor drie visuele processen, namelijk viseel bewustzijn, visuele discriminatie en priming, op hetzelfde moment. Na inachtnemening van de data verkregen door stimulatie van het controle hersengebied, verscheen deze overlap ook in het pre-stimulus domein. Met placebo TMS werden echter andere resultaten behaald. Bovendien verschilden de niet-neurale TMS effecten per taak. Dit roept de methodologische vraag op welke TMS controleconditie de voorkeur verdient binnen welke experimentele context.

Het volgende project (Hoofdstuk 4) onderzocht de rol van vroege visuele hersengebieden in een hogere cognitieve functie, te weten het kortetermijngeheugen. TMS-pulsen werden toegediend op een variabel tijdsinterval tijdens de retentieperiode van een geheugentaak, die het visuele kortetermijngeheugen (VSTM) aanwendt. Bij zware belasting van het kortetermijngeheugen, werden proefpersonen minder accuraat, als de TMS puls 200ms na afloop van de te onthouden plaatjes volgde. Dit 'memory-masking' effect trad alleen op wanneer de TMS werd toegediend aan de contralaterale hersenhelft ten opzichte van het visuele veld waarin de stimuli verschenen. Gelijkaardige resultaten werden behaald in een vervolgexperiment waarin een visuele stimulus in plaats van 
een TMS-puls gebruikt werd om de geheugenrepresentatie te maskeren. Tezamen duiden deze bevindingen aan, dat een ongehinderde verwerking in de EVC vereist is voor het onthouden van visuele stimuli in het kortetermijngeheugen, op een bepaald moment en alleen voor informatie gepresenteerd in het contralaterale gezichtsveld. Omdat de puls te laat komt om de 'feedforward'-verwerking te verstoren, betekent dit dat de occipitale TMS-puls 'feedback' van hogere (visuele) hersengebieden verstoort. Mogelijk treedt deze terugkeer van informatie op, zodat er voortdurend een vergelijking plaats kan vinden tussen de huidige input van de EVC en de informatie die op hetzelfde moment verwerkt wordt in visuele hersengebieden buiten de EVC.

De in Hoofdstuk 2 tot 4 gerapporteerde resultaten tonen aan dat de EVC functioneel relevant is voor verscheidene visuele processen op bepaalde momenten, zowel in het pre- als het post-stimulus domein. Het verwachte neurale mechanisme dat ten grondslag ligt aan het pre-stimulus TMS effect is waarschijnlijk anders dan dat van het post-stimulus TMS effect. Kennis over deze mechanismen kan meer inzicht geven in de exacte verwerkingsprocessen die in de EVC plaatsvinden tijdens visuele waarneming. Om hier meer over te weten te komen is het belangrijk om TMS te combineren met een andere onderzoeksmethode die direct de hersenactiviteit meet. Aangezien de gevonden TMS effecten alleen op een specifieke tijd plaatsvinden, is de combinatie met electroencefalografie (EEG), een methode met een zeer hoge temporele resolutie, het meest voor de hand liggend. Maar, het verkrijgen van EEG-data tijdens een TMS onderzoek blijkt technisch moeilijk realiseerbaar. Zoals in Hoofdstuk 5 beschreven, hebben we de meest optimale TMS-EEG set-up voor het Maastricht Brain Imaging Center geselecteerd en hiermee vervolgens EEGdata verzameld tijdens een TMS 'masking' experiment, waarin visuele perceptie onderdrukt werd door TMS-pulsen over de EVC. Tijdens de pre-stimulus trials, was het EEG-signaal veroorzaakt door de visuele stimulus, het zogenoemde 'Visual Evoked Potential' (VEP), volledig onzichtbaar door het veel grotere TMS artefact. Desalniettemin, lukte het om VEPs met een normale amplitude en gelijkend op de VEPs tijdens trials zonder TMS te verkijgen. Dit werd bereikt door de gemiddelde EEG respons na een TMS-puls te middelen en deze af te trekken van de experimentele data. Toekomstige analyses zullen zich richten op de vergelijking van VEPs tussen trials waarbij wel versus geen subjectieve waarneming van de stimulus door de proefpersoon gerapporteerd werd.

Er worden in dit proefschrift meerdere suggesties gedaan voor toekomstig onderzoek. Zo is de vraag of wederkerige circuits doorslaggevend zijn voor visueel bewustzijn nog niet definitief beantwoord en is verder onderzoek hiernaar vereist. Het neurale mechanisme dat de gedragsmatige invloed van sensorische stimuli reguleert, het neuraal correlaat van subliminale priming (NCSP) verdient ook verdere wetenschappelijke aandacht. Tot slot is het vanuit een methodologisch perspectief essentieel om nader te onderzoeken wat, gegeven de context van het onderzoek, een optimale controle voor de nietneurale bijwerkingen van TMS zou kunnen zijn. 

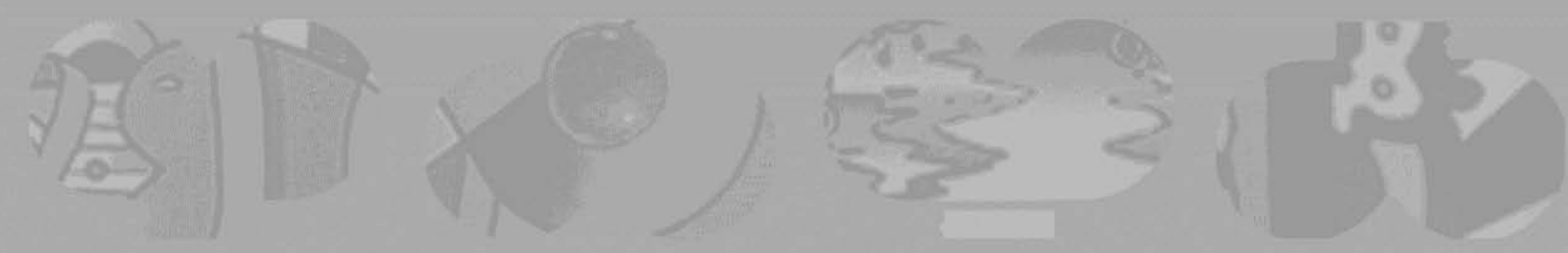

Acknowledgments
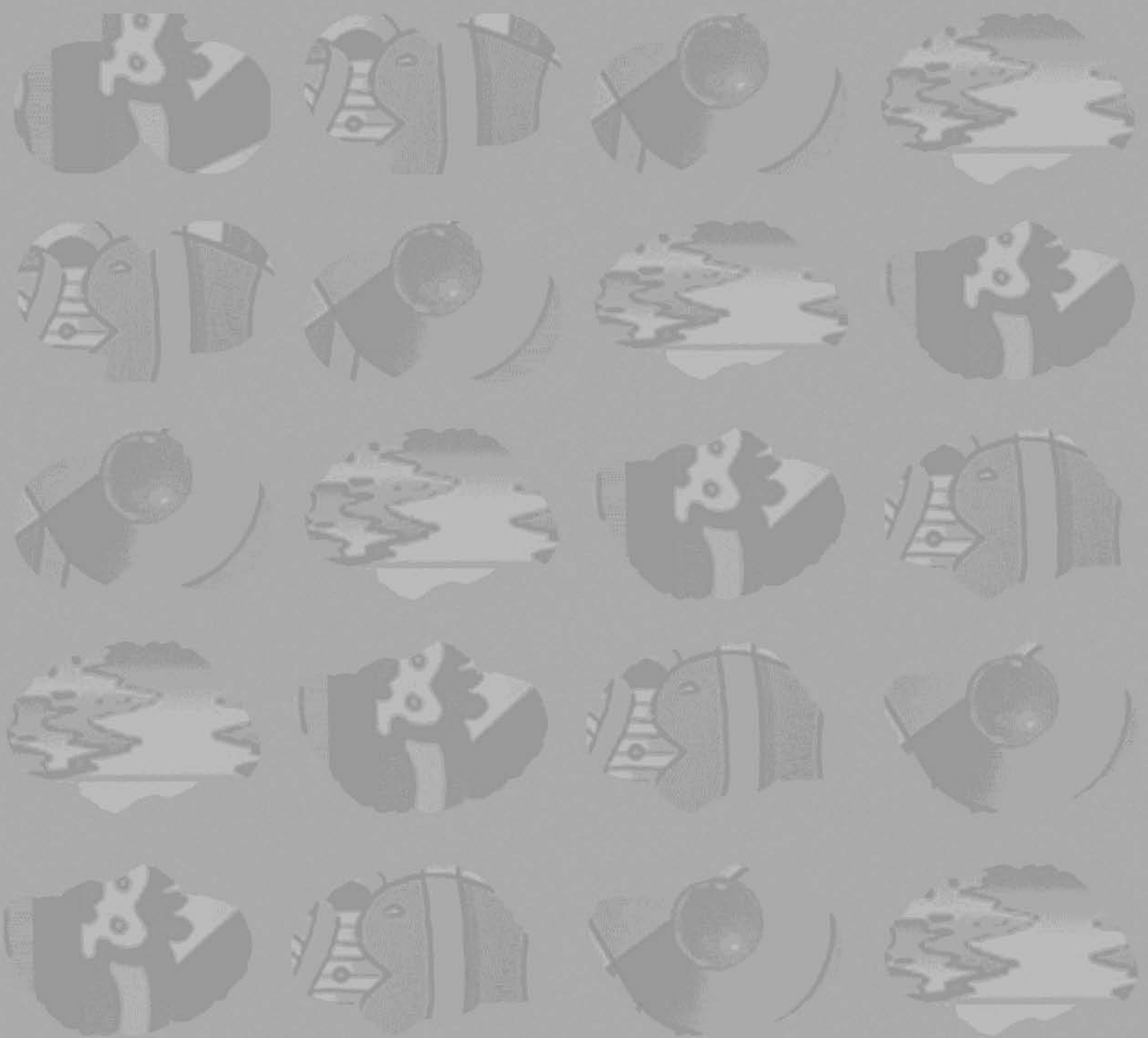
Het schrijven van een dankwoord geeft je de gelegenheid om de afgelopen jaren en de mensen die in deze periode het verschil gemaakt hebben te overzien. De mensen om me heen zijn enorm insprirerend en hebben me enorm veel geleerd, zowel op professioneel als persoonlijk vlak.

First of all, Alex. Thanks for the faith you had in me! When I was your student assistant, you showed me how cool research can be, and so convinced me to do a PhD project (something I wasn't sure of at the time). In hindsight I am very happy that you did, especially because I enjoyed your great supervision. I really appreciate the freedom you gave me, and still I always felt that you had my back if necessary.

Rainer, head of the amazing CN department and my second promotor. During my research internship I already got to know your contagious enthusiasm for research, which is truly inspiring. Thanks for creating opportunities!

Many thanks to all the colleagues (and by now, friends) in the TMS group. Our meetings start late, last too long and the meeting room becomes more crowded every time... Still, their open character, inspirational presentations, birthday treats, and funny conversations make them into valuable moments, contributing to the atmosphere of cooperation and companionship that I experience within our group. Alex, Teresa, Nina, Tom, Felix, Rosanne, Franziska, Vincent, Tomasso, Jeanette, Thamar and all past members of the TMS group (particularly Joel!), thanks for that. We rock!

Felix, you're the only creature alive that talks as much as I do! Still, somehow someone decided to put the two of us in one office... But I must say, our chats are most enjoyable and very fruitful (if we talk about work at least (:)), and your open and warm personality make you a very pleasant person to be around. I actually think we talk so much, because we share a general interest in, well, everything. Anna, thanks for putting up with us! And thank you, Felix, Anna, Julien and Sven for putting up with my continuous singing and the annoying habit I have of talking to myself.

Thanks to all the (student) assistants I got to work with over the years. Bastian, Tahnée, Jeroen, Sonja, Danique, Anette and Michelle, you've been a great help! Many of you also participated in (all of my) studies. In am very grateful to you and all the other participants for giving me the precious data to work with!

Joel, Marin, Peter, Tom en Vincent, ik heb enorm veel van jullie geleerd en veel plezier beleefd aan onze samenwerking! Bedankt daarvoor.

Bedankt Annemie, Christl en Riny voor allerhande ondersteuning op zoveel gebieden. En voor de gezellige gesprekjes in het secretariaat! Ook Erik bedankt voor het enorme aantal fotodiodemetingen dat je voor me hebt gedaan en René voor de snelle hulp bij labaangelegenheden.

Arie, Henk, Jan, Manuela, Peter, Tom and leader-of-our-pack Rob, you make philosophy fun! Our discussions have helped me to better understand the complexity and (im)possibilities of studying consciousness. Thanks for challenging me! 
Noël, zonder jou zou dit proefschrift er nooit gekomen zijn. Waarschijnlijk weet je het zelf niet eens, maar jij gaf me eens heel goed advies.

And some colleagues are just nice to hang out with, making FPN such a wonderful place to spend your days: Sanae, Joost, Judith, Pim, and the other (former) fifth floor 'inhabitants', thanks for the lunches, coffee breaks, Friday afternoon beers, movie nights etc. Rilana en Ken, op meer pizza's en koffies samen op weg naar jullie proefschriften! We moeten wel wat verder lopen nu....

De hechte relatie tussen studie, werk en privéleven wordt heel duidelijk in de personen van Nina en Saskia, mijn paranimfen. Na samen te hebben gestudeerd, hebben we alledrie besloten om nog een tijdje te blijven en om samen het avontuur van een promotietraject aan te gaan. Ook al hebben we alledrie een eigen verhaal, niemand weet zo goed hoe het is om een proefschrift te schrijven als jullie. En niemand kent mijn persoonlijke verhaal beter dan jullie. Saskia, de uni is een stuk minder kleurrijk geworden nu jij er niet meer rondloopt, maar gelukkig kan ik nog regelmatig genieten van je positieve levenshouding en je extreme openmindedness. Nina, onze wegen lopen wel nog parallel, nu zelfs rechtstreeks naar hetzelfde kantoor. Ik bewonder je relaxte manier van zijn en vind het een voorrecht dat ik die nu van nog dichterbij mag meemaken. Dames, bedankt dat jullie achter me willen staan.

Ik heb nog meer contacten met lieve mensen aan mijn studietijd overgehouden. Helen, Marieke, Yvonne en Rob, de gezamenlijke etentjes (en de bijbehorende glaasjes wijn) zijn een stuk minder frequent, maar daarom niet minder gezellig geworden! Grappig hoe een serieus gesprek en een slappe lach zo dicht bij elkaar kunnen liggen!

Ellen, nog zo iemand die (voor nu) Limburg achter zich heeft gelaten. Het moet lastig zijn voor onze omgeving (lees: Emile en Stefan) wanneer we samen zijn, twee van die druktemakertjes bij elkaar. Heel erg bedankt voor de enorme positieve energieboost die ik krijg van alles wat we samen ondernemen. Ik waardeer het enorm dat we altijd eerlijk tegen elkaar (kunnen) zijn.

De meiden die het traject dat leidde tot dit proefschrift het langst van dichtbij hebben meegemaakt zijn Emmy, Frederiek, Irene, Mirthe en Sandra. Er is een eindeloze rij aan hoogte- en dieptepunten die we sinds die eerste 'Girls Only' samen hebben meegemaakt. Bedankt voor alle steun die ik gekregen heb tijdens het bereiken van dit persoonlijke hoogtepunt in de vorm van telefoontjes, berichtjes en zelfs thuisbezorgde warme maaltijden. Ook al was er in de laatste fase van de afronding nog bar weinig respons mijnerzijds, weet dat jullie gewaardeerd werden en worden. $\mathrm{Na}$ al die jaren voelen jullie (en jullie mannen) als familie. Hopelijk mag onze familie de komende jaren nog groeien; het begin is gemaakt!

Anke, hier sta je dan, en terecht! Als ik ziek ben $(-)$, maar zeker ook in goede gezondheid zie ik je (on)aangekondigde pitstops bij ons huis met plezier tegemoet. Ik weet dat je het soms moeilijk vindt dat jullie leven zo anders is dan dat van ons sinds de komst van de terroristjes (jouw woorden), maar ik verheug me al op het moment dat Floor en Jurre meegaan met onze etentjes. Freek, ik besef dat jij hier niet zo van houdt, dus ik hou het kort: bedankt! 
En natuurlijk is er hier ook een plekje gereserveerd voor Andre en Ilse. Jullie ondernemingszin en mentale veerkracht is een enorme inspiratiebron, waar ik nog vaak van hoop te mogen proeven. Ik gun jullie alle moois voor de toekomst!

Zo zie je maar hoe sport mensen bij elkaar brengt, en sportclub BC Smash is hier bijzonder goed in. No mercy op de badmintonbaan, maar daarna gezellig samen een biertje drinken bij Vic. Het laatste jaar van mijn promotietraject is dat biertje, euh partijtje badminton, er te vaak bij ingeschoten, maar gelukkig had ik in de eerste drie genoeg fysieke fitheid opgebouwd om me door dat laatste jaar heen te slaan. BC Smash, bedankt! In het bijzonder Chris (en Kim), Didier (en Michelle), Kirsten, Lars, Mariska, Mark, Robert, en Sander: ik weet dat ik de Starfish vaker voorstel dan dat we er echt heen gaan, maar het zegt zeker iets over de goede sfeer waarin onze feestjes plaatsvinden. Ik verwacht dat jullie vlammen op 28 september!

Het leuke aan schoonfamilie is dat ze anders is dan de vertrouwde omgeving waar je in bent opgegroeid en dat ze je zo een nieuwe kijk biedt op alledaagse zaken. Zo weet ik inmiddels waar Houthem St.-Gerlach ligt en wat een accuboormachine is. Maar afgezien van dit soort weetjes heb ik inmiddels ook van jullie geleerd dat schoonfamilie heel vertrouwd kan voelen. Ellen en Jacques, Sander en Mariska, bedankt daarvoor en voor alle gezellige dineetjes met mijn lievingskostje friet zuurvlees!

Oma Mai, ik vind het heel bijzonder dat je deze mijlpaal mag meemaken. Eindelijk afgestudeerd, zoals je zelf zou zeggen. Ook al is het dit keer niet bedoeld als cabaret, ik hoop dat je op 28 september toch geniet van mijn 'opvoering'. Het voelt fijn om je weer in de zaal erbij te hebben. Ik hoop dat de andere opa's en oma's er toch ook een beetje bij zijn vandaag

Kleine pröpkes worden groot. Lieve mam en pap, jullie hebben me altijd gestimuleerd om zoveel mogelijk uit mezelf te halen, zeker ook op academisch gebied. Jullie hebben op deze manier het fundament gelegd waarop dit proefschrift is gebouwd. Het doet me goed te weten dat jullie trots op me zijn, maar wat mij betreft mogen jullie dat zeker ook op jezelf zijn!

Mijn grote broer mag natuurlijk niet ontbreken in deze lijst. Vroeger dacht ik altijd dat we verschrikkelijk anders waren, terwijl ik er steeds meer achterkom dat we eigenlijk zoveel op elkaar lijken. Ook al woon je in het 'verre' Amsterdam, je komt steeds een beetje dichterbij. Lieve Ralf, bedankt voor alle support en oprechte interesse in alles wat me bezighoudt.

Stefan, jij vond dat de mensen die het belangrijkst zijn de meeste tekst verdienen in dit dankwoord. Omdat ik het de lezers niet aan wil doen om nog 140 bladzijden door te ploegen (en omdat ik denk dat ik je eigenlijk wel weet wat je voor me betekent), beperk ik me tot het volgende: lieve Stefan, ik klaag altijd dat ik geen echt 'passie' in mijn leven heb, maar dat is eigenlijk niet waar. In je eigen woorden: "you are what makes me tick". 

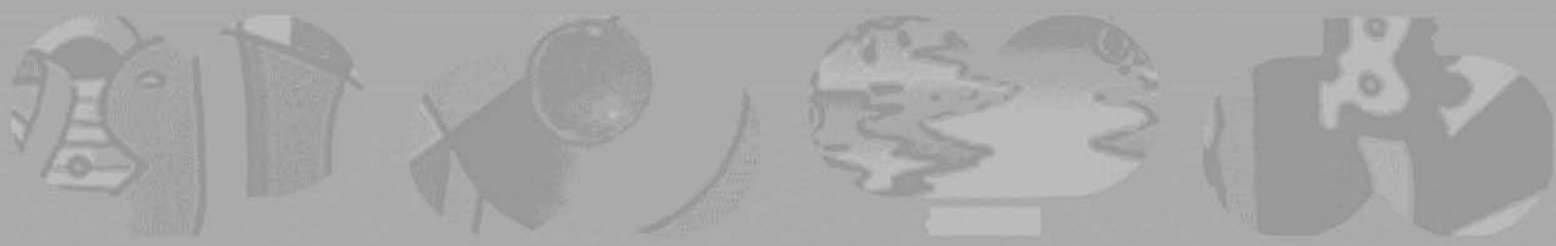

Curriculum Vitae
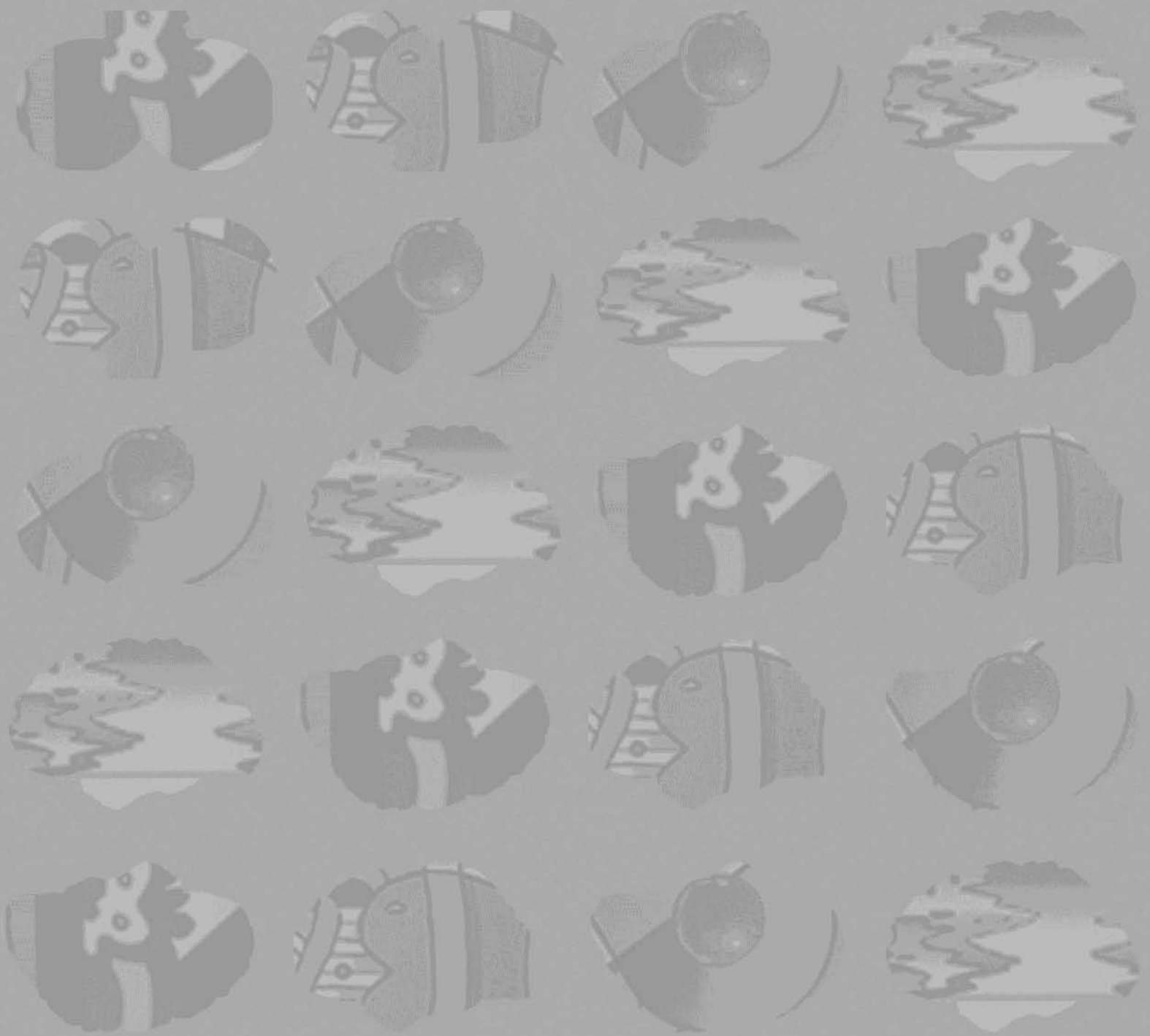
Christianne Julienne Henriëtte Jacobs was born on the 31th of August, 1983 in Maastricht, the Netherlands. In 2001, she finished her pre-university education at the Sint Maartenscollege, Maastricht. In September, Christianne started as a Psychology student at Maastricht University. She studied abroad for five months in the period July-December 2004 at the University of Sydney, New South Wales, Australia. In June 2006, she graduated cum laude in Biological Psychology (Neuropsychology and Cognitive Neuroscience tracks). After a short study period in Nijmegen (Political Sciences), Christianne started as a research assistant in the Transcranial Magnetic Stimulation (TMS) group of Alexander Sack at Maastricht University in April 2007. She started her PhD project in January 2008 within the same group under supervision of prof. dr. Alexander Sack and prof. dr. Rainer Goebel. Christianne will continue to work in the group of professor Sack as a post-doctoral fellow.

\section{Peer-reviewed journal articles}

Jacobs, C., \& Sack, A.T. (2012). Behavior in Oblivion: the neurobiology of subliminal priming. Brain Sciences, 2(2), 225-241.

Jacobs, C., Goebel, R., \& Sack, A. T. (2012). Visual awareness suppression by pre-stimulus brain stimulation; a neural effect. Neuroimage, 59(1), 616-624.

De Weerd, P., Reithler, J., van de Ven, V., Been, M., Jacobs, C., \& Sack, A. T. (2012). Posttraining transcranial magnetic stimulation of striate cortex disrupts consolidation early in visual skill learning. The Journal of Neuroscience, 32(6), 1981-1988.

van de Ven, V., Jacobs, C., \& Sack, A. T. (2012). Topographic contribution of early visual cortex to short-term memory consolidation: a transcranial magnetic stimulation study. The Journal of Neuroscience, 32(1), 4-11.

de Graaf, T. A., Cornelsen, S., Jacobs, C., \& Sack, A. T. (2011). TMS effects on subjective and objective measures of vision: stimulation intensity and preversus post-stimulus masking. Consciousness and cognition, 20(4), 12441255.

de Graaf, T. A., Jacobs, C., Roebroeck, A., \& Sack, A. T. (2009). FMRI effective connectivity and TMS chronometry: complementary accounts of causality in the visuospatial judgment network. PLoS One, 4(12), e8307.

Sack, A. T., Jacobs, C., De Martino, F., Staeren, N., Goebel, R., \& Formisano, E. (2008). Dynamic premotor-to-parietal interactions during spatial imagery. The Journal of Neuroscience, 28(34), 8417-8429. 


\section{Journal articles in preparation}

Jacobs, C., de Graaf, T. A., Goebel, R., \& Sack, A. T. (2012). The temporal dynamics of early visual cortex involvement in behavioral priming PLoS ONE, In Revision 
